\title{
Spezielle Therapieformen
}

P.L. Bölcskei, M. Dreher, S. Ellis, L. Freitag, P. Haidl, M. Höper, A.-M. Kirsten,

D. Köhler, T. Kramm, H. Magnussen, H. Matthys, H.-J. Schäfers, J. Storre, T. Welte, W. Windisch

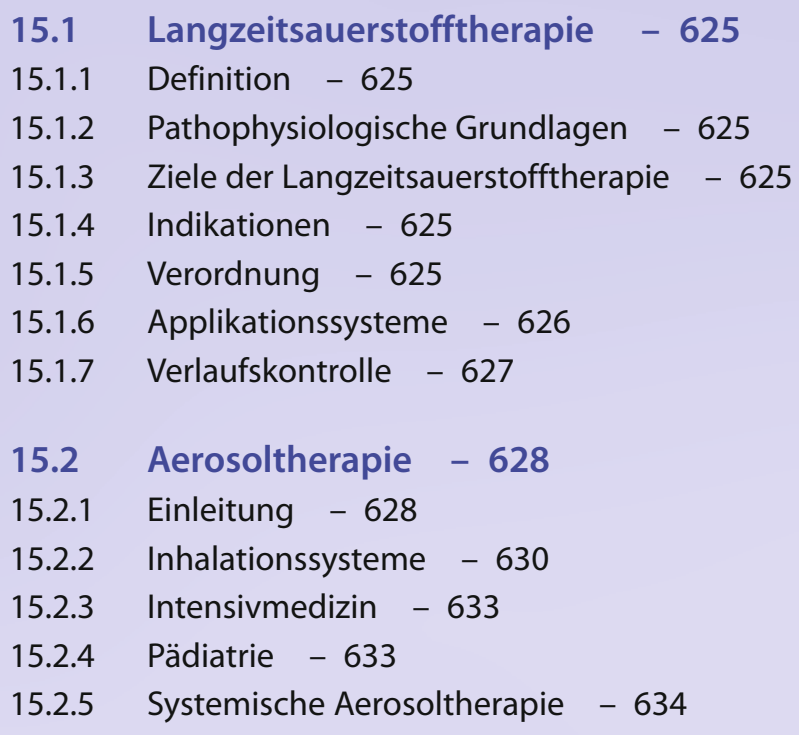

15.3 Nichtinvasive Beatmung - Therapie der Atempumpinsuffizienz - 635

15.3.1 Grundlagen der nichtinvasiven Beatmung - 635

15.3.2 Nichtinvasive Beatmung bei akuter respiratorischer Insuffizienz - 640

15.3.3 Nichtinvasive Beatmung bei schwieriger Entwöhnung vom Respirator, während der Postextubationsphase und perioperativ - 643

15.3.4 Nichtinvasive Beatmung bei chronischer respiratorischer Insuffizienz - 644

$15.4 \quad$ Maschinelle Beatmung -649

15.4.1 Einleitung - 649

15.4.2 Indikationen zur maschinellen Beatmung - 649

15.4.3 Grundlagen der Beatmung - 651

15.4.4 Beatmungsmodi - 654

15.4.5 Beatmung bei speziellen Erkrankungen - 657

15.4.6 Entwöhnung vom Respirator (Weaning) - 659

15.4.7 Supportive Konzepte - 660

15.4.8 Lungenersatzverfahren - 662 
15.5 Lungentransplantation - 667

15.5.1 Einleitung - 667

15.5.2 Empfängerauswahl und Entscheidungskriterien - 667

15.5.3 Organspenderkriterien -669

15.5.4 Organkonservierung -670

15.5.5 Transplantationstechnik - 670

15.5.6 Postoperatives Management - 671

15.5.7 Spezifische Komplikationen - 671

15.6 Interventionelle thorakale Endoskopie - 676

15.6.1 Vorbemerkung - 676

15.6.2 Bronchoskopie - 676

15.6.3 Thorakoskopie - 685

15.7 Atemkrankheiten und Schwangerschaft - 689

15.7.1 Bedeutung von Atemkrankheiten in der Schwangerschaft - 689

15.7.2 Asthma bronchiale - 689

15.7.3 Pneumonien - 689

15.7.4 Lungentuberkulose - 690

15.7.5 Pulmonale Hypertonie - 690

15.7.6 Lungenembolien - 690

15.7.7 Mukoviszidose - 690

15.7.8 Lungenfibrosen - 690

15.7.9 Schlafapnoesyndrom - 690

15.7.10 Fliegen und Tauchen - 691

15.8 Tabakentwöhnung - 691

15.8.1 Möglichkeiten der Tabakkontrolle - 691

15.8.2 Verhaltens- und soziale Aspekte der Tabakabhängigkeit - 691

15.8.3 Diagnostik der Motivation zum Rauchstopp und der Tabakabhängigkeit - 692

15.8.4 Behandlung der Tabakabhängigkeit - 693

15.8.5 Verschiedene Therapiesettings - 695

15.8.6 Medikamentöse Therapie der Tabakabhängigkeit - 696

15.8.7 Spezielle Populationen - 698 


\subsection{Langzeitsauerstofftherapie}

\subsubsection{Definition}

Jede Sauerstoffgabe, die länger als $15 \mathrm{~h} /$ Tag über mehr als 3 Monate indiziert ist, bezeichnet man als Langzeitsauerstofftherapie (»long term oxygen therapy«, LTOT).

\subsubsection{Pathophysiologische Grundlagen}

Der positive Effekt der Langzeitsauerstofftherapie auf die Symptomatik, Lebensqualität, Morbidität und Mortalität der behandelten Patienten ist gut belegt. Der pathophysiologische Mechanismus, der diese günstige Wirkung erklärt, ist nicht sicher bekannt. Sauerstoff führt beim Vorliegen einer Hypoxämie zu einer Abnahme der Atemarbeit. Da die meisten Patienten, bei denen eine Langzeitsauerstofftherapie indiziert ist (COPD), eine erhöhte Atemarbeit haben, ist die sauerstoffinduzierte Reduktion der Atemarbeit eine nachvollziehbare Erklärung für den klinischen Effekt. Die Langzeitsauerstofftherapie beeinflusst die pulmonale Hämodynamik insbesondere bei Höhenaufenthalten. Daten einer französischen retrospektiven Untersuchung über 10 Jahre (ANTADIR-Obervatory) zeigten bei Patienten mit COPD unter Langzeitsauerstofftherapie folgende Risikofaktoren: Männliches Geschlecht, Alter, niedriger BMI, FEV1 (\% vom Sollwert). $\mathrm{P}_{\mathrm{a}} \mathrm{O}_{2}$ und $\mathrm{P}_{\mathrm{a}} \mathrm{CO}_{2}$ sind unabhängige negative Prognosefaktoren. Ein niedriger Hämatokrit ist ebenfalls mit einer erhöhten Morbidität und Mortalität assoziiert.

\subsubsection{Ziele der Langzeitsauerstofftherapie}

Patienten mit Erkrankungen, die mit einer chronischen Hypoxämie einhergehen, weisen eine verminderte Lebensqualität und Leistungsfähigkeit auf und haben eine erhöhte Morbidität und Mortalität. Die Ziele der Langzeitsauerstofftherapie sind eine Verbesserung der Lebensqualität und Leistungsfähigkeit sowie eine Reduktion von Morbidität und Mortalität (• Abb. 15.1).

Die Lebenserwartung wird bei chronisch hypoxämischen Patienten mit chronisch-obstruktiver Bronchitis und Lungenemphysem (COPD) durch die Langzeitsauerstofftherapie verlängert. Die Langzeitsauerstofftherapie hat bei männlichen Patienten einen geringeren Effekt auf die Mortalität als bei Frauen.

\subsubsection{Indikationen}

Die Indikation zur Langzeitsauerstofftherapie setzt diagnostische Möglichkeiten voraus, die in der Regel den

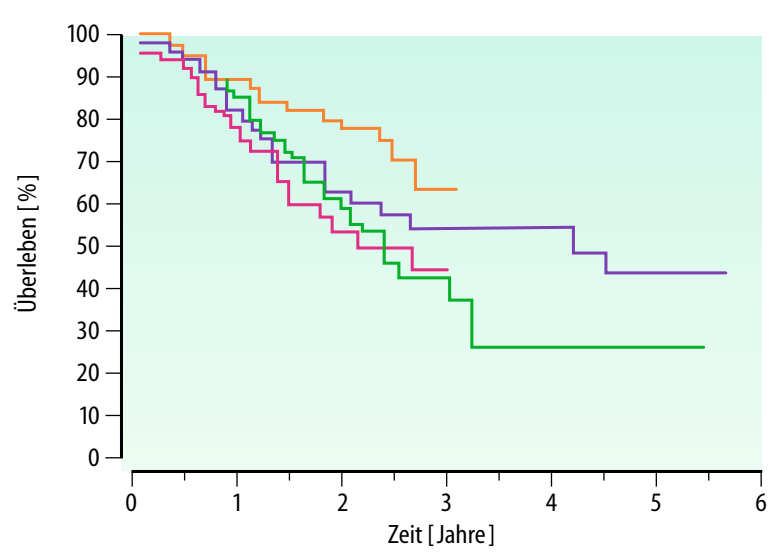

- Abb. 15.1. Überleben bei Patienten mit COPD nach Beginn einer Langzeitsauerstofftherapie. Orange $\mathrm{O}_{2}$-Therapie $24 \mathrm{~h} / \mathrm{Tag}$, blau $15 \mathrm{O}_{2}$ Therapie

Pneumologen in Klinik und Praxis zur Verfügung stehen. Die Indikationsstellung sollte in Übereinstimmung mit internationalen Empfehlungen durch den Spezialisten erfolgen.

Die Indikation zur Langzeitsauerstofftherapie ist gegeben, wenn nach adäquater Therapie und Vermeidung aller inhalativen Noxen eine chronische Hypoxämie nachweisbar ist (• Abb. 15.2). Die Kooperation des Patienten sollte gegeben sein. Eine asymptomatische Hyperkapnie unter Langzeitsauerstofftherapie stellt keine Kontraindikation dar.

Eine behandlungsbedürftige chronische Hypoxämie liegt vor, wenn der arterielle Sauerstoffpartialdruck $\left(\mathrm{P}_{\mathrm{a}} \mathrm{O}_{2}\right)$ unter Ruhebedingungen während einer stabilen Krankheitsphase von ca. 4 Wochen mehrfach (mind. 3-mal) unter $55 \mathrm{mmHg}(7,3 \mathrm{kPa})$ lag. Die Indikation ist auch bei einem $\mathrm{P}_{\mathrm{a}} \mathrm{O}_{2}$ von $55-60 \mathrm{mmHg}$ und gleichzeitigem Cor pulmonale oder einer Polyglobulie gegeben. Eine Indikation zur Sauerstofftherapie besteht weiterhin bei einem Abfall des $\mathrm{P}_{\mathrm{a}} \mathrm{O}_{2}$ unter $55 \mathrm{mmHg}$ unter Belastung oder während des Schlafs.

\subsubsection{Verordnung}

Die Verordnung einer Langzeitsauerstofftherapie hat Folgendes zu berücksichtigen:

- Diagnose,

- Schweregrad der Hypoxämie und Hyperkapnie,

- Sauerstofffluss zum Erreichen eines $\mathrm{P}_{\mathrm{a}} \mathrm{O}_{2} \geq 60 \mathrm{mmHg}$ $(8 \mathrm{kPa})$ oder Anstieg um mindestens $10 \mathrm{mmHg}$,

- Mobilität des Patienten,

- persönliche Präferenz für das Applikationssystems,

- stabile Phase der Erkrankung,

- Rauchverhalten, 


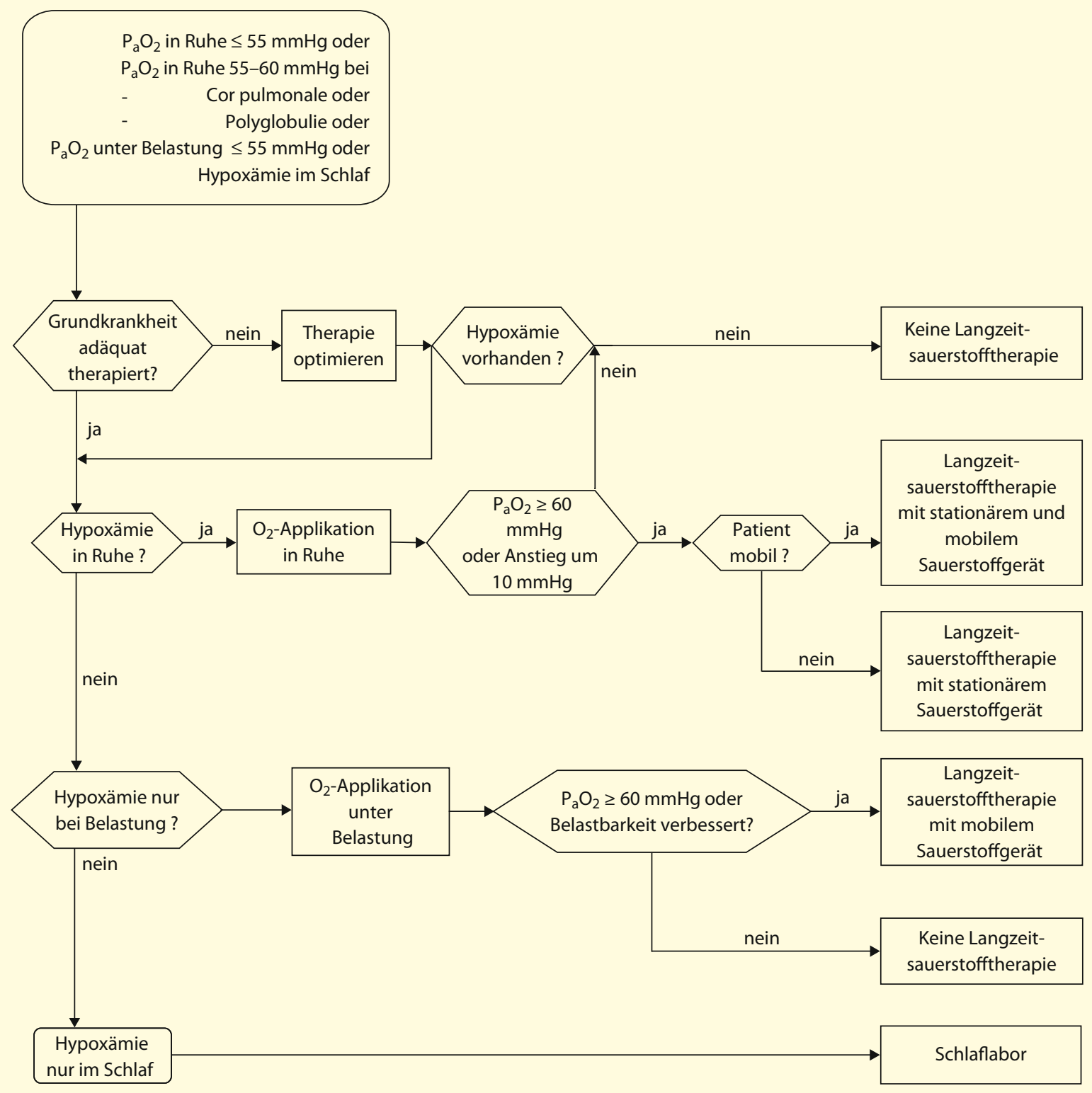

- Abb. 15.2. Algorithmus zur Langzeitsauerstofftherapie

- Bereitschaft zur >15-stündigen Anwendung,

- bei Belastungshypoxämie: Bereitschaft zur Anwendung des tragbaren Systems,

- Reevaluation in 3-6 Monaten.

\subsubsection{Applikationssysteme}

Für die häusliche und mobile Sauerstofftherapie stehen als Quellen Sauerstoffkonzentratoren, Flüssigsauerstoffsysteme und Sauerstoffdruckflaschen zur Verfügung.
Sauerstoffkonzentratoren. Sauerstoffkonzentratoren absorbieren mittels Kompressor fortlaufend Sauerstoff aus der Umgebungsluft. Die Geräte sind großvolumig, haben ein Gewicht von ca. 18-30 kg und benötigen eine Stromzufuhr (120-450 W). Daher können sie nur stationär, unter häuslichen Bedingungen eingesetzt werden.

Tragbare Konzentratoren. Eine Neuentwicklung ist der Minikonzentrator mit einem Gewicht von $2 \mathrm{~kg}$. Er kann mit einem Batteriegürtel verwendet werden (Gewicht 
0,8 kg). Damit steht erstmals ein Sauerstoffkonzentrator für den mobilen Einsatz (2 h) zur Verfügung. Nachteile des Geräts sind der geringe Sauerstoffluss (31/min) sowie die störende Geräuschentwicklung. Die Entwicklung von Konzentratoren, die Brennstoffzellentechnologie anwenden, muss abgewartet werden.

Flüssigsauerstoffsysteme. Das Flüssigsauerstoffsystem benötigt ein häusliches Standgerät als Reservoir. Dieses muss vom Gashersteller direkt mit flüssigem Sauerstoff befüllt werden, sodass derartige Systeme eine entsprechende Logistik voraussetzen. Aus dem häuslichen Tank füllt der Patient einen tragbaren Behälter ab. Das Gewicht der gefüllten Sauerstoffbehälter beträgt je nach Volumen 3,1-4,3 kg. Bei einem Sauerstoffbedarf von $21 / \mathrm{min}$ liefert das tragbare System Sauerstoff für ca. 3-4h. Der Verbrauch von Sauerstoff wird durch elektronische Sparventile bzw. getriggerte Demandsysteme verringert. Transportsysteme in Form einer Rückentragehilfe oder Caddy haben sich bewährt. Die tragbaren Sauerstoffsysteme sind die Voraussetzung für die Behandlung der belastungsabhängigen Hypoxämie.

Demandsysteme. Sparsysteme, die als zusätzliches Gerät am mobilen Flüssigsauerstoffsystem befestigt werden, haben sich nicht bewährt. Demandsysteme sind heute meist integriert und können die Mobilität mit nur einer einzigen Füllung und einem Gewicht des mobilen Tanks von nur $2,5 \mathrm{~kg}$ von 7 auf $20 \mathrm{~h}$ erhöhen.

Sauerstoffdruckflaschen. Sauerstoffdruckflaschen sind in unterschiedlichen Größen verfügbar. Der Nachteil liegt in ihrem großen Gewicht und der Logistik. Sie sind nicht für die Langzeitsauerstofftherapie geeignet.

\section{Sauerstoffapplikation}

In der Regel wird der Sauerstoff über die Nase appliziert. Die gebräuchlichsten und von den Patienten am besten akzeptierten Systeme sind die Nasenbrille, oder auch die Nasensonde. In Brillengestelle zu integrierende Systeme haben kosmetische Vorteile und fördern die Compliance. Eine Alternative kann ein transtrachealer Katheter sein, der neben dem Vorteil der für Außenstehende unsichtbaren Sauerstoffgabe die Totraumventilation vermindert und in kritischen Fällen zu besseren Sauerstoffkonzentrationen führen kann. Nachteilig sind die Notwendigkeit eines Eingriffs, die Gefahr einer Obstruktion der Trachea durch Schleimpfropfen am Katheter oder durch Infektion sowie der erhöhte Pflegebedarf mit täglichem Katheterwechsel.

\subsubsection{Verlaufskontrolle}

Die Kontrolle der Patienten unter Langzeitsauerstofftherapie soll im stabilen Zustand alle 3-6 Monate durch einen Pneumologen erfolgen. Exazerbationen der Grunderkrankungen müssen ursächlich abgeklärt werden und bedingen wegen der therapeutischen Konsequenz häufigere Kontrollen. Bei jeder Kontrolle ist

- der klinische Zustand des Patienten zu dokumentieren,

- Nebenwirkungen zu erfragen und zu minimieren,

- die Therapie zu optimieren,

- ein mögliches Fehlverhalten (z. B. Inhalationsrauchen) zu korrigieren,

- die Nutzung des Gerätes zu prüfen.

\section{Literatur}

Calverley PM, Walker P (2003) Chronic obstructive pulmonary disease. Lancet 362:1053-1061

Celli B, MacNee W (2004) Standards for the diagnosis and treatment of patients with COPD: a summary of the ATS/ERS position paper. Eur Respir J 23(6):932-946

Chailleux E, Fauroux B, Binet F, Dautzenberg B, Polu JM for the observatory group of ANTADIR (1996) Predictors of survival in patients receiving domiciliary oxygen therapy or mechanical ventilation. Chest 109:741-749

Chambellan A, Chailleux E, Similowski T, ANTADIR Observatory group (2005) Prognostic value of the hematocrit in patients with severe COPD receiving long-term oxygen therapy. Chest 128(3):1201-1208

Croxton TL, Bailey WC for the NHLBI working group on long-term oxygen treatment in COPD (2006) Long term oxygen treatment in chronic obstructive pulmonary disease: recommendations for future research - an NHLBI workshop report. Am J Respir Crit Care Med 174:373-378

Eaton T, Garrett JE, Young P et al. (2002) Ambulatory oxygen improves quality of life of COPD patients: a randomised controlled study. Eur Respir J 20(2):306-312

Global Strategy for diagnosis, management and prevention of chronic obstructive pulmonary disease (2006) www.goldcopd.com

Gorecka D, Gorzelak K, Sliwinski P et al. (1997) Effect of long-term oxygen therapy on survival in patients with chronic obstructive pulmonary disease with moderate hypoxaemia. Thorax 52(8):674-679

Lacasse R, Lecours C, Pelletier R, Begin, Maltais F (2005) Randomised trial of ambulatory oxygen in oxygen-dependent COPD. Eur Respir J 25(6):1032-1038

Magnussen $\mathrm{H}$ et al. (2001) Leitlinien zur Langzeit-Sauerstofftherapie. Pneumologie 55:454-464

Machado MC, Krishnan JA, Buist SA et al. (2006) Sex differences in survival of oxygen-dependent patients with chronic obstructive pulmonary disease. Am J Respir Crit Care Med 174(5):524-529

Martis S, Munos X, Rios J et al (2006) Body weight and comorbidity predict mortality in COPD patients treated with oxygen therapy. Eur Respir J 27(4):689-696

Medical Research Council Working Party (1981) Long term domiciliary oxygen therapy in chronic hypoxic cor pulmonale complicating chronic bronchitis and emphysema. Lancet 1:681-686

Nocturnal Oxygen Therapy Trial Group (1980) Continuous or nocturnal oxygen therapy in hypoxaemic chronic obstructive lung disease. Ann Intern Med 93:391-398

Wijkstra PJ, Guyatt PH, Ambrosino N et al. (2001) International approaches to the prescription of long-term oxygen therapy. Eur Respir J 18(6):909-913 


\subsection{Aerosoltherapie}

\section{P. Haidl, D. Köhler}

\subsubsection{Einleitung}

Die Einführung eines treibgasbetriebenen Dosieraerosols im Jahre 1956 war der Katalysator für die Verbreitung der Aerosoltherapie in der Behandlung insbesondere obstruktiver Atemwegserkrankungen. Das Verbot der fluorierten Kohlenwasserstoffe (FCKW), 1987 im Montrealer Protokoll vorgeschlagen, führte zu einem weiteren Entwicklungsschub der Inhalationssysteme. In Deutschland betrug zuletzt das Verordnungsvolumen für Medikamente in Aerosolform über 400 Millionen Euro pro Jahr.

Entscheidende Vorteile einer topischen Therapie in den Atemwegen bestehen im raschen Wirkungseintritt bei einer im Vergleich zur oralen Gabe deutlich geringeren Dosis. Dies führt zu einer Senkung der Nebenwirkungsrate. Neben dem therapeutischen Einsatz werden Aerosole zu diagnostischen Zwecken (spezifische und unspezifische nasale und bronchiale Provokation, Lungenventilationsszintigraphie, Messung der mukoziliären und resorptiven Clearance) verwendet.

\section{Grundlagen der Aerosoltherapie}

Aerosole stellen ein Zweiphasensystem dar, in dem sich feste oder flüssige Partikel mit einem aerodynamischen Durchmesser zwischen $1 \mathrm{~nm}$ und $100 \mu \mathrm{m}$ in einem gasförmigen System (Luft) bewegen. In einem therapeutischen Aerosol liegen die Partikel in unterschiedlichen Größen vor (polydisperses Aerosol). Zur Kennzeichnung der Verteilung der Partikelgröße wird der massenbezogene mediane aerodynamische Durchmesser (MMAD) verwendet. Dies ist der Teilchendurchmesser eines Teilchenkollektivs, bei dem 50\% der Masse aller Teilchen größer und 50\% kleiner sind als dieser Wert. Zusätzlich wird die Streuung der Partikelgröße durch die geometrische Standardabweichung (GSD) beschrieben. Der MMAD der gängigen Inhalationssysteme bewegt sich zwischen $1 \mu \mathrm{m}$ und $6 \mu \mathrm{m}$. Die Hersteller verzichten leider auf Angaben zum Partikelspektrum in der jeweiligen Produktinformation.

Partikel über $10 \mu \mathrm{m}$ sind nicht lungengängig; sie werden in der Nase oder im Oropharynx abgeschieden. Die Wahrscheinlichkeit der Lungendeposition erreicht bei Teilchen um 0,5 $\mu \mathrm{m}$ ein Minimum, da diese Teilchen zum Großteil wieder exhaliert werden. Werden die Teilchen noch kleiner (z. B. ultrafeine Partikel), steigt die Depositionswahrscheinlichkeit wieder an.

Die Masse eines Aerosolteilchens ist abhängig von der dritten Potenz des Durchmessers. Das bedeutet, dass ein 5 - $\mu \mathrm{m}$-Partikel im Vergleich zu einem 1- $\mu \mathrm{m}$-Partikel die 125-fache Medikamentenmasse tragen kann.

\section{Depositionsmechanismen}

Impaktion (Massenträgheit). Sie betrifft bevorzugt Teilchen über $5 \mu \mathrm{m}$. Große und damit träge Partikel können bei hoher Geschwindigkeit einer Richtungsänderung des Luftstroms nicht folgen und prallen auf die Atemwegswand. Die Impaktion ist der Hauptmechanismus der Deposition im Oropharynx und tracheobronchial. Die Deposition ist direkt proportional zur Geschwindigkeit und zum Quadrat der Teilchengröße des Aerosols.

Sedimentation (Schwerkraft). Sie betrifft bevorzugt Teilchen zwischen 1 und $5 \mu \mathrm{m}$. Diese Teilchen dringen tiefer in das Bronchialsystem ein, d.h. sie können den Richtungsänderungen an den Bifurkationen folgen. Im Verlauf des Atemmanövers sinken die Partikel schwerkraftabhängig und können Wandkontakt erhalten. Die Sedimentation ist direkt proportional zur Zeit und zum Quadrat der Teilchengröße. Daher begünstigen die Länge der Inspiration sowie die Atemanhaltezeit am Ende der Inspiration neben der Teilchengröße die Lungendeposition durch Sedimentation.

Diffusion. Sie betrifft bevorzugt Teilchen unter $1 \mu \mathrm{m}$. Diese Partikel erreichen insbesondere den Alveolarraum. Wandkontakt entsteht durch die Brown'sche Molekularbewegung, d.h. durch zufällige Kollisionen der Teilchen mit Ablenkung zur Alveolarwand. Der Großteil dieser Partikel wird wieder exhaliert. Die Deposition ist proportional zur Zeit und der geometrischen Teilchengröße. Wie oben erwähnt beschreibt die Depositionswahrscheinlichkeit sehr kleiner Partikel eine U-förmige Kurve, d.h. die Deposition nimmt mit weiter abnehmender Partikelgröße wieder zu (• Abb. 15.3).

\section{Atemmanöver}

Aerosole können eine hohe inter- und weniger intraindividuelle Variabilität (»Hub zu Hub«) in Hinblick auf die Quantität und die Qualität der Lungendeposition aufweisen (『Abb. 15.4). Diese Schwankungen können durch ein langsames und tiefes Einatmen vermindert werden. Dieses Atemmanöver eignet sich daher besonders für Dosieraerosole und Vernebler.

Eine Ausnahme hiervon stellen die meisten Pulverinhalatoren dar, die zur erforderlichen Desagglomeration des eingefüllten Pulvers eine rasche und forcierte Inhalation benötigen. Hintergrund ist dabei, dass während der ersten $200 \mathrm{~ms}$ der Inspiration ein Großteil des Aerosols aus dem System freigesetzt wird.

Da sich die Atemmanöver bei Dosieraerosolen und Trockenpulversystemen prinzipiell unterscheiden, sollten die Systeme nicht gleichzeitig bei einem Patienten verwendet werden. Anderenfalls besteht ein erhöhter Schulungsaufwand. Dagegen ist aber zu berücksichtigen, dass 

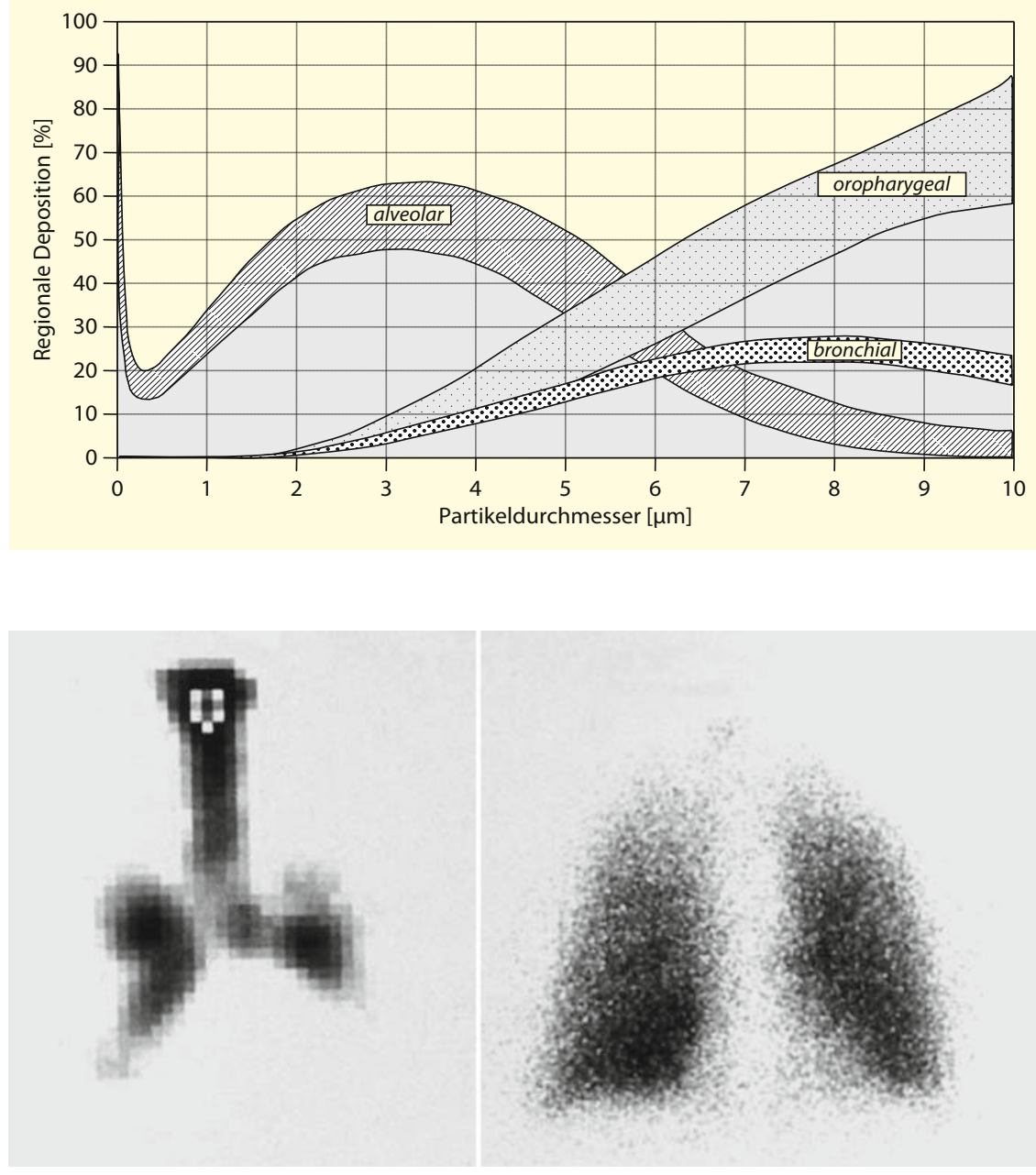

Flache, schnelle Atmung MAMD $7 \mu \mathrm{m}$

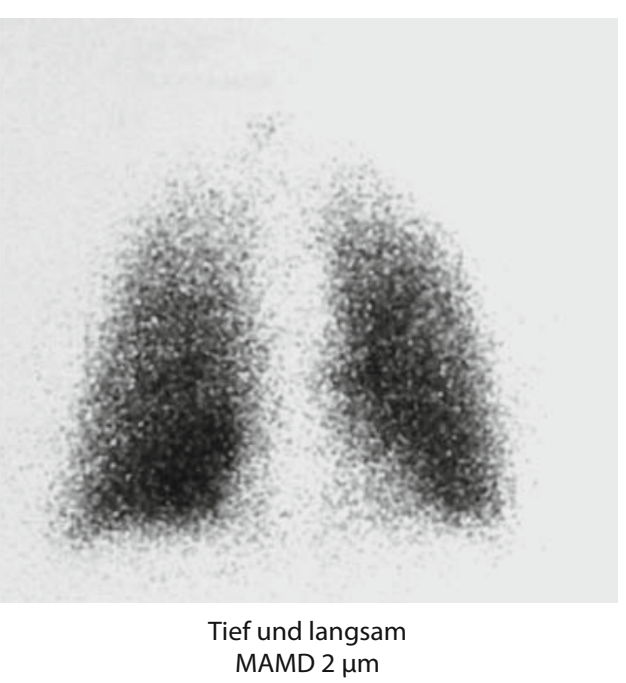

- Abb. 15.3. Abhängigkeit der regionalen pulmonalen Aerosoldeposition vom Partikeldurchmesser. Die dunklen Bänder stellen die interindividuelle Abweichung dar
- Abb. 15.4. Einfluss der Teilchengröße und des Atemmanövers auf die Lungendeposition. Aufnahmen nach Inhalation eines Radioaerosols im akuten schweren Asthmaanfall oder bei akuter Luftnot eines COPD-Patienten mit deutlich reduzierten Inspirationsflüssen nur ein Dosieraerosol ggfs. mit Hohlraumsystem (»Spacer») zuverlässig verwendet werden kann.

Bei Jugendlichen und Erwachsenen ist die Inhalation über die Nase grundsätzlich zu vermeiden, da die Nase als Filter 90\% der Partikel zurückhält.

\section{Atemwegsanatomie}

Die interindividuelle Variabilität der pulmonalen und oropharyngealen Deposition wird durch anatomische Unterschiede in der Rachen- und Glottisregion mitverursacht (• Abb. 15.5). Bei einem Patienten z. B. mit einer schweren schlafbezogenen Atmungsstörung mit engem Hypopharynx und Hufeisenepiglottis wird mit hoher Wahrscheinlichkeit die Rachendeposition deutlich höher und die Lungendeposition niedriger ausfallen. Je kleiner die Aerosolpartikel sind $(<3 \mu \mathrm{m})$, desto geringer wird allerdings die Bedeutung der anatomischen Unterschiede. Weiterhin beeinflusst die Atemwegserkrankung die regionale Verteilung der Lungendeposition. Mit zunehmender Bronchialobstruktion wird die Deposition nach zentral in die großen Atemwege verlagert; die Absolutmenge bleibt aber unverändert. In nicht ventilierten Lungensegmenten (z. B. mit Sekret verlegte Bronchien oder Emphysemblasen) werden keine Partikel abgeschieden.

\section{Zusammenfassung}

Die Lungendeposition hängt von der Partikelgröße des Aerosols, dem Massenausstoß des Inhalationsgerätes, der Art der Lungenerkrankung, der individuellen Atemwegsgeometrie und als wichtigstem Faktor von dem Atemmanöver des Patienten ab. Die klinische Wirkung und die Nebenwirkungsrate bleiben der Maßstab, ob die einzelnen Einflussfaktoren erfolgreich zusammenwirken. 
- Abb. 15.5. Endoskopische Aufnahmen der Glottisregion, die die anatomische Variabilität zeigen
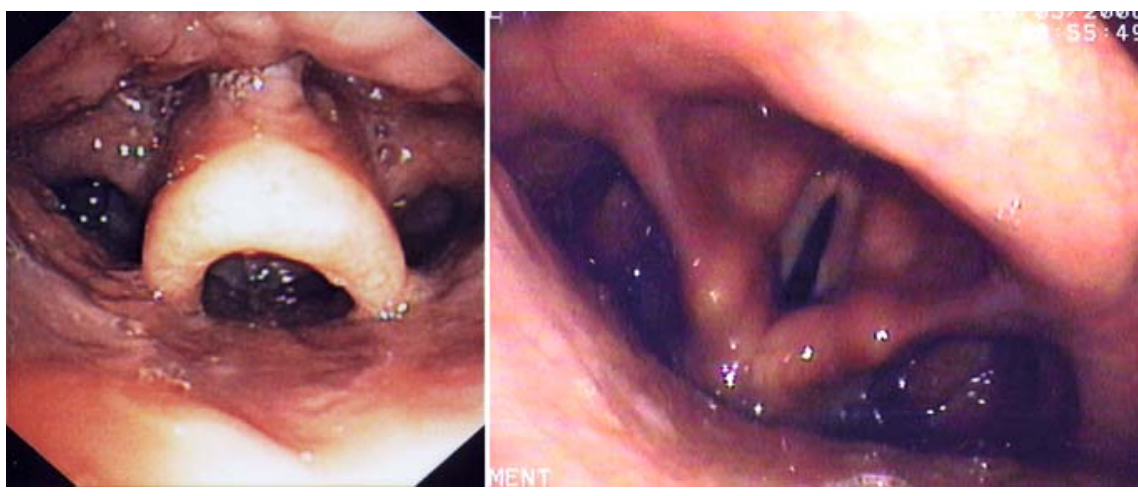

\subsubsection{Inhalationssysteme}

Es stehen derzeit 3 verschiedene Gruppen an Inhalationssystemen zur Verfügung:

- treibgasbetriebene Dosieraerosole (pMDI, "pressurized metered dose inhaler «),

- Trockenpulverinhalatoren (DPI, »dry powder inhaler«),

- Düsen- und Ultraschallvernebler.

\section{Dosieraerosole}

Das Prinzip besteht aus der Mischung eines Medikaments mit einem Treibgas (in der Regel Hydrofluoralkan HFA 134a und HFA 227) als Suspension oder Lösung in einem unter Druck stehenden Metallkanister. Bei Druck auf den Behälter gelangt der in einer Dosierkammer befindliche Wirkstoff in Kontakt mit der Austrittsdüse und das Aerosol wird mit unterschiedlich hoher Geschwindigkeit freigesetzt (bis $30 \mathrm{~m} / \mathrm{s}$ ). Diese hohe Geschwindigkeit sowie die anfangs noch nicht erfolgte Verdampfung des Treibgases sind für die meist hohe Rate der oropharyngealen Deposition (70-90\% der etikettierten Dosis) durch Impaktion verantwortlich. Lösungsaerosole mit kleiner Partikelgröße von $1-2 \mu \mathrm{m}$ reduzieren diesen Mechanismus. Gleichzeitig ist die korrekte Abfolge von Beginn der Inspiration und gleichzeitiger Sprühstoßauslösung von großer Bedeutung und bereitet einem relevanten Teil der Patienten Probleme.

Hohlraumsysteme mit Ventil (holding chamber, auch als Spacer bezeichnet) sind in der Lage, sowohl die Koordination zu erleichtern als auch die Mund-RachenDeposition auf unter 10\% der Dosis zu reduzieren. Nach Applikation jeweils eines Sprühstoßes in das Hohlraumsystem sollte der Patient innerhalb von 1-2 s aus diesem inhalieren. Hohlraumsysteme sollten bei der Inhalation von Kortikosteroiden zur Anwendung kommen, um die typischen Nebenwirkungen Mundsoor und Heiserkeit zu minimieren. Ausnahmen sind Inhalationssysteme mit kleiner Partikelgröße (1-2 $\mu \mathrm{m})$ und Steroide, die als Prodrug wie Ciclesonide erst in der Bronchialschleimhaut aktiviert werden.
Vorteile der Dosieraerosole sind die Robustheit, die niedrigen Kosten und die hohe und konstante Dosisfreisetzung von über $90 \%$ der eingefüllten, etikettierten Dosis.

Die Dosis und das Partikelspektrum sind außerdem unabhängig vom Atemfluss des Patienten und können daher (insbesondere in Verbindung mit einem Hohlraumsystem) auch im schweren Asthmaanfall und bei Patienten mit schwerer Atemwegsobstruktion angewendet werden.

Eine weitere Option zur Umgehung der Koordinationsprobleme stellt das sog. Autohaler-Prinzip dar, die atemzuggetriggerte Freisetzung des Aerosols. Der Kanister wird unter eine Vorspannung gesetzt und der Patient beginnt zu inhalieren. Bei Überschreiten eines Inspirationsflusses von 30 1/min wird eine Klappe geöffnet und das Aerosol freigesetzt.

Eine Sonderstellung nimmt der Respimat ${ }^{\bullet}$ ein, der eigentlich ein mechanischer Vernebler für Flüssigkeiten ist und als Doppelstrahlimpaktionsinhalator bezeichnet wird. Mithilfe eines Federmechanismus wird die Medikamentenlösung unter hohem Druck durch zwei v-förmig angeordnete Siliziumdüsen gepresst. An der Spitze treffen die beiden Aerosolstrahlen aufeinander und erzeugen durch Impaktion ein sehr feines Aerosol. Auf eine sonst bei Verneblern verwendete Prallplatte kann dadurch verzichtet werden. Das Aerosol zeichnet sich durch eine niedrige Austrittsgeschwindigkeit $(0,8 \mathrm{~m} / \mathrm{s})$ und eine lange im Raum stehende Sprühwolke (bis 1,5s) aus, die die Koordination für den Patienten erleichtert.

\section{Trockenpulverinhalatoren}

Der Großteil der neu auf dem Markt eingeführten Inhalationssysteme sind Pulverinhalationssysteme, die auf Treibgase verzichten. Der entscheidende Faktor für die Freisetzung des Aerosols ist hier die Inspirationskraft bzw. der Atemfluss des Patienten. Der Wirkstoff liegt in Pulverform in einer Kapsel bzw. einem Blister oder in einem Reservoir vor. - Abb. 15.6 gibt einen Überblick über die derzeit erhältlichen Trockenpulverinhalatoren. 


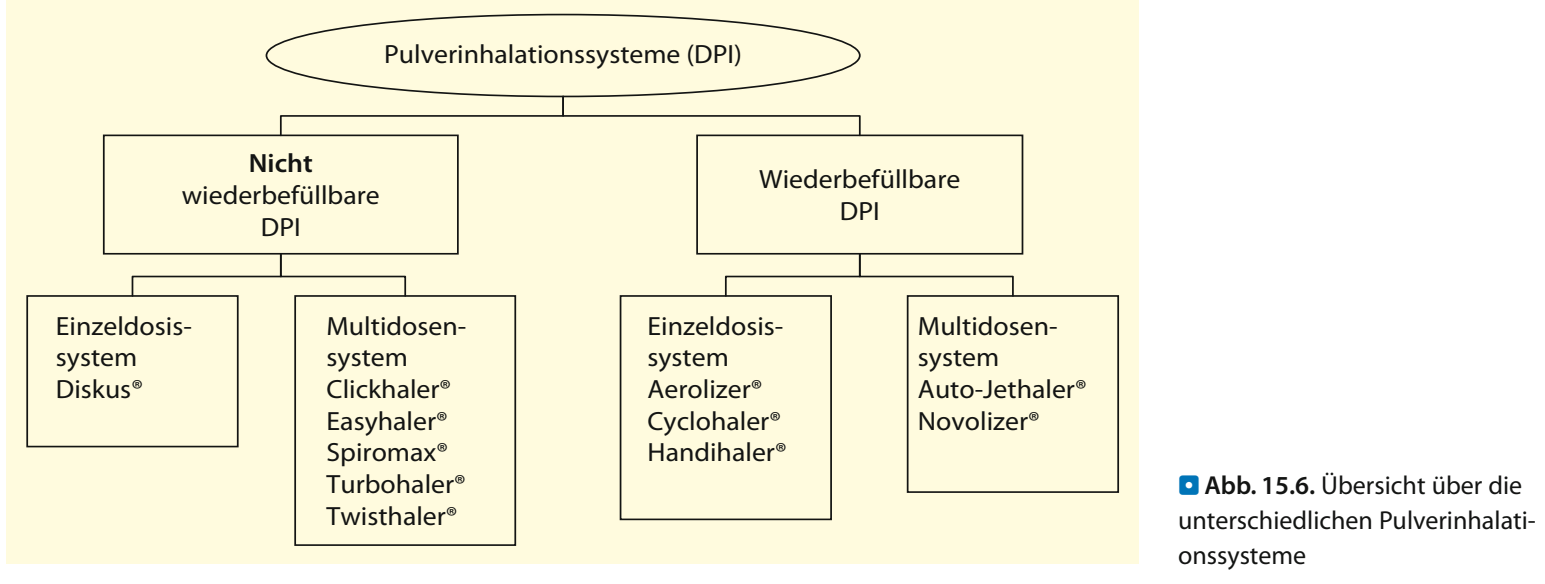

Das Medikament in Pulverform bildet Agglomerate in nicht respirabler Größe. Durch die Inspiration wird über einen unterschiedlich ausgeprägten inneren Gerätewiderstand eine hohe Geschwindigkeit erzeugt, die zur Desagglomeration des Pulvers und der Abspaltung der zugemischten Laktose führt. Damit werden respirable Partikel $(\mathrm{MMAD}<5 \mu \mathrm{m})$ generiert. In der Folge variiert die Partikelgröße und die intrapulmonal deponierte Dosis in Abhängigkeit von der Stärke des Inspirationsflusses des Patienten, die über den "peak inspiratory flow" (PIF) abgeschätzt werden kann. Der in der Spirometrie gemessene PIF wird ohne Systemwiderstand erzielt. Um eine Vergleichbarkeit mit der Angabe »die Flussrate über das Inhalationssystem sollte $30-60 \mathrm{l} / \mathrm{min}$ betragen « $\mathrm{zu}$ erreichen, muss der individuelle Gerätewiderstand berücksichtigt werden. Beispielsweise bedeutet dies für den Turbohaler ${ }^{\oplus}$ mit hohem inneren Widerstand, dass spirometrisch ein PIF von ca. $280 \mathrm{l} / \mathrm{min}$ bzw. 4,31/s erreicht werden muss, um einen Atemfluss durch den Turbohaler von $60 \mathrm{l} / \mathrm{min}$ zu erzielen. Mit niedrigerem Gerätewiderstand wird der Korrekturfaktor kleiner.

Ein wichtiger Vorteil der DPI besteht darin, dass eine Koordination zwischen Inspiration und Bereitstellung des Aerosols nicht notwendig ist. Die Atempause am Ende der Inspiration spielt bei Pulvern (Inhalation von Steroiden) eine geringere Rolle als bei den pMDI, da das Pulver während der Inspiration hygroskopisch wächst und damit schneller sedimentiert.

Von Bedeutung ist, dass der Patient zunächst wie bei einem Vitalkapazitätsmanöver in der Lungenfunktion maximal exspiriert, um nachfolgend die Relaxationskräfte der Exspirationsmuskulatur zum Erreichen eines hohen Inspirationsflusses auszunutzen. Bei schwerer Überblähung oder Obstruktion sind hier aber Grenzen gesetzt. Der erforderliche maximale Inspirationsfluss muss möglichst innerhalb der ersten $200 \mathrm{~ms}$ der Inspiration erzielt werden. Nur in dieser Zeit wird das Aerosol aus dem System freigesetzt, d.h. nur dann führt der hohe Fluss zur Verbesserung der Desagglomeration mit nachfolgend erhöhter Zahl kleiner Partikel.

Ein sinnvolles Prinzip stellt das Überwinden eines Mindestflusses dar, bevor ein Federmechanismus mit Rückmeldung (optisch und akustisch) die korrekte Inhalation auslöst. Ansonsten könnte sich der Patient bei niedrigen Inspirationsflüssen $(<30 \mathrm{l} / \mathrm{min}$ distal der gerätebedingten Stenose) in falscher Sicherheit wiegen. Laktosepartikel im Mund geben zwar das Gefühl einer erfolgreichen Inhalation. Ein großes Laktosemolekül erlaubt aber keine Aussage darüber, ob wirklich respirable Medikamentenpartikel die Lunge erreicht haben.

\section{Vernebler}

- Tab. 15.1 listet die Vor- und Nachteile der Vernebler im klinischen Einsatz auf. Herauszuheben ist die Anwendung bei Substanzen, die nicht »von der Stange « in einem pMDI oder DPI verfügbar sind. Hierzu zählen insbesondere Ilomedin bei primär pulmonaler Hypertonie, Ciclosporin nach Organtransplantation, Pentamidin (Prophylaxe von Pneumocystis carinii), Substanzen zur Verbesserung der bronchialen Clearance (Salzlösungen, ACC, Ambroxol) und Antibiotika, die bei der zystischen Fibrose und bei Patienten mit Pseudomonas-besiedelten Bronchiektasen zur Anwendung kommen.

Weiterhin haben Vernebler ihren Einsatzbereich bei Kindern und älteren Menschen mit Koordinationsproblemen oder neurologischen bzw. motorischen Defiziten (z. B. rheumatoide Arthritis, Tremor, Myopathie). Im akuten Asthmaanfall können $\beta$-Mimetika oder Adrenalin, bei einem Pseudokrupp Steroide und Adrenalin inhaliert werden.

Zudem werden modifizierte Vernebler mit Mess- und Steuereinrichtungen in der pneumologischen Diagnostik verwendet (z. B. spezifische oder unspezifische bronchiale Provokation, s. $>$ Kap.7.1, Abschn.7.1.5). Daher sind Grundkenntnisse der Systeme wichtig. Die Standardisie- 
- Tab. 15.1. Vor- und Nachteile der Vernebler

Vorteile

- Keine Koordination erforderlich

- Einsatz bei Kindern und geriatrischen Patienten

- Einsetzbar für Arzneimittellösungen, die weder in DPI noch in $\mathrm{PMDI}$ verfügbar sind

- Kombination unterschiedlicher Wirkstoffe möglich

- Einsatz in Notfallsituationen

- In Beatmungssystemen einsetzbar

\section{Nachteile}

- Größe und Notwendigkeit einer Energiequelle

- Regelmäßige Reinigung erforderlich

- Lange Inhalationsdauer

- Anschaffungskosten

- Hohe Restmenge (effizientere Systeme sind in der Entwicklung) rung des Atemflusses und des Atemzugvolumens tragen dazu bei, große inter- und intraindividuelle Schwankungen zu reduzieren. Damit werden unerwünschte und bedrohliche Zwischenfälle durch eine zu hohe Lungendeposition der Provokationssubstanz vermieden.

Mit einem Marktanteil über $80 \%$ werden Düsenvernebler verwendet. Ein Kompressor liefert Druckluft, die über eine Düse im Verneblerkopf nach dem Venturi-Prinzip einen Unterdruck erzeugt, der ein sog. Primäraerosol erzeugt. Über Prallplatten kann dann die Tröpfchengrösse des Aerosols, das letztlich das Mundstück verlässt, weiter modifiziert werden. Als guter Kompromiß hat sich die kontinuierliche Verneblung unter Verwendung von Ventilen im In- und Exspirationsschenkel bewährt (• Abb. 15.7).

Je nach Hersteller und Anwendungsdauer sollten die Vernebler alle 1-2 Jahre ausgetauscht werden. Durch die Desinfektion und die Materialalterung verändert sich die Beschaffenheit der Düse und die Zahl der respirablen Partikel nimmt ab. Je teurer die zu vernebelnde Substanz, umso bedeutsamer ist dieses Phänomen. Die Kompressoren haben eine Lebensdauer von deutlich über 5 Jahren.

Eine Alternative stellen Ultraschallvernebler dar. Ein Piezo-elektrischer Kristall erzeugt Schallwellen im MHz-Bereich, die an der Oberfläche wässriger Lösungen ein "Abreißen« von Wassermolekülen zur Folge haben. Durch dieses Prinzip kommt es auch zu einer Erwärmung des Aerosols während des Betriebs. Dies wird von einer Reihe Patienten als angenehm empfunden, andererseits kann dies bei der Inhalation von Proteinen (DNAse, Prolastin) zur Denaturierung führen. Die Lebensdauer der Ultraschallgeräte wird meist durch Kalkablagerungen auf dem Ultraschallschwinger begrenzt.

Ein drittes und neues Prinzip stellen die "vibrating mesh «-Vernebler dar (z. B. PARI e-flow ${ }^{\bullet}$, Aeroneb $\left.{ }^{\star}\right)$. Ein ringförmiger Piezo-elektrischer Kristall, auf dem eine perforierte Membran sitzt, wird elektronisch in Schwingung versetzt. Ein alternierendes Druckfeld wird aufgebaut, die Medikamentenflüssigkeit durch die feinen Poren gepresst und das Aerosol erzeugt.

Generell sollte der Patient zur topischen pulmonalen Therapie über ein Mundstück inhalieren. Nasenmasken senken über die Filterwirkung der Nase die Lungende-
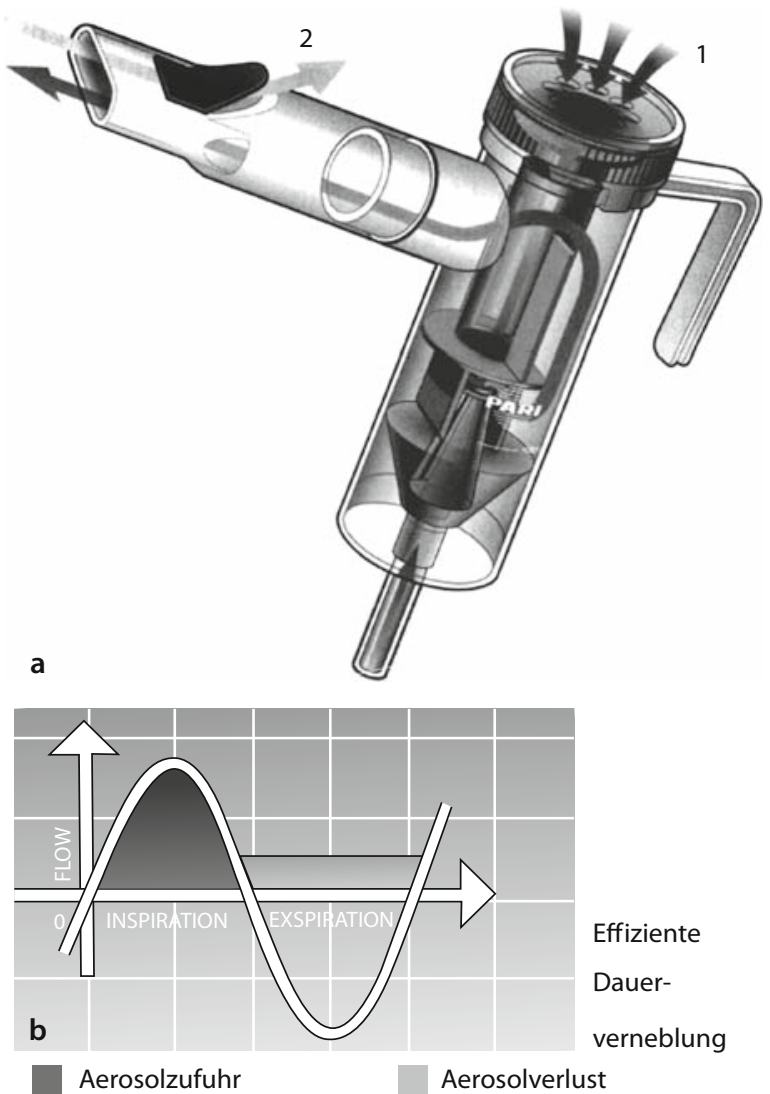

- Abb. 15.7a,b. Atemzuggesteuerte Aerosolinhalation bei Spontanatmung. a Durch den inspiratorisch entstehenden Unterdruck wird das vom Vernebler kontinuierlich gebildete Aerosol durch die Öffnung des Inspirationsventils (1) mit Raumluft verdünnt eingeatmet. Bei der Exspiration wird dieses Ventil durch den dabei entstehenden Überdruck geschlossen und das Ausatmungsventil (2) geöffnet. b Im Vergleich zur atemzuggetriggerten Inhalation wird die Inhalationszeit praktisch halbiert bei geringer Steigerung des Aerosolverlusts in der Exspiration

position drastisch. Die Angaben des Herstellers zur Füllmenge sind zu beachten.

In der Bewertung eines Verneblers ist sowohl der Massenausstoß pro Zeiteinheit als auch die Zahl der respirablen Partikel zu berücksichtigen (i. d. R. angegeben als Anteil der Partikel $<5 \mu \mathrm{m}$ ); häufig wird ein Kompromiss zwischen beiden Größen erforderlich sein. 


\section{Auswahl eines Inhalationssytems}

Für die Aerosoltherapie stehen die 3 dargestellten Systemgruppen mit ihren Vor- und Nachteilen zur Verfügung. Die Übersicht führt die wichtigsten Fragen auf, die der Arzt vor der Verordnung eines Inhalationssystems beantworten sollte. Aus den Antworten ergeben sich dann das oder die geeigneten Systeme.

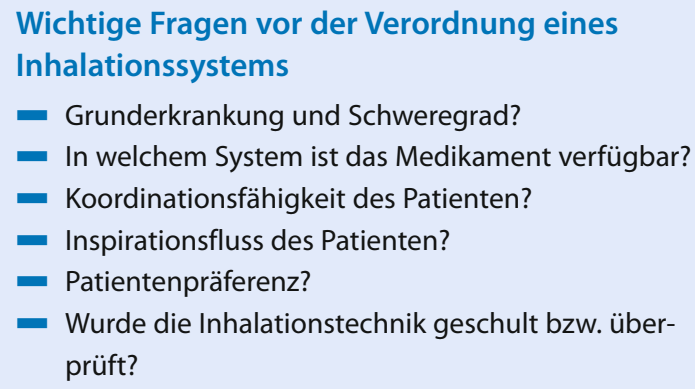

\subsubsection{Intensivmedizin}

Auf einer Intensivstation sind die 3 nachfolgenden Situationen zu unterscheiden, in denen eine Aerosoltherapie infrage kommen kann. Im Allgemeinen liegt die Lungendeposition, die mit einem pMDI mit Hohlraumsystem oder einem Vernebler bei beatmeten Patienten erzielt wird, bei $5-15 \%$ der etikettierten Dosis bzw. der Füllmenge. pMDI haben Vorteile, da die Applikationsdauer kürzer ist und ein Hohlraumsystem ständig im Beatmungssystem belassen werden kann. Damit entfallen wiederholte Diskonnektionen.

Spontanatmender Patient. Je nach zu verabreichender Substanz kommen pMDI möglichst mit Hohlraumsystem oder ein Vernebler zur Anwendung. Düsenvernebler können hierbei mit $\mathrm{O}_{2}$ betrieben werden (Flussmenge 5-61/min). DPI sind bei den in der Regel respiratorisch limitierten Patienten nicht zu empfehlen. Die Kombination einer nichtinvasiven Maskenbeatmung mit einem Vernebler erhöht die pulmonale Deposition nicht.

Intubierter Patient ohne Spontanatmung. Für die Gabe eines $\beta$-Mimetikums oder eines Steroids sollte als erste Präferenz ein Hohlraumsystem im Abstand von $30 \mathrm{~cm}$ vom Tubus in den Inspirationsschenkel des Beatmungsschlauchs eingebracht werden (• Abb. 15.8). Die manuelle Auslösung des pMDI durch den Anwender erfolgt am Ende der Exspiration. Zuvor sollte das Atemzugvolumen auf über $500 \mathrm{ml}$ erhöht werden und ggf. eine Befeuchtung unterbrochen werden. Düsen- oder Ultraschallvernebler sind die Alternative für die Applikation z. B. von Ilomedin oder Antibiotika, die an der gleichen Stelle wie das Hohlraumsystem einge-

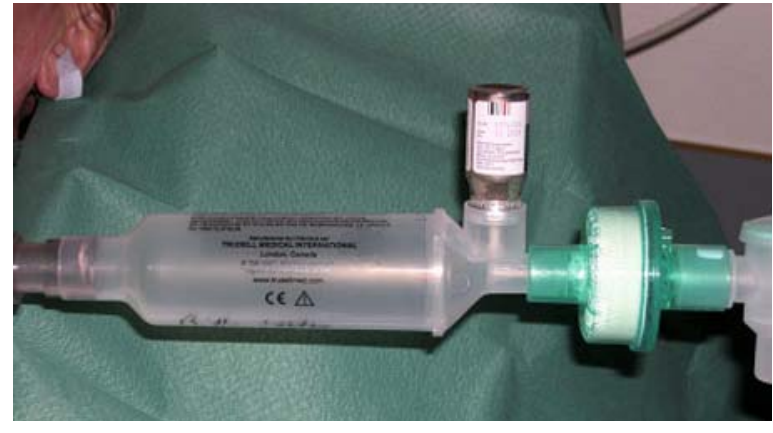

- Abb. 15.8. Hohlraumsystem mit Dosieraerosol im Beatmungsschlauch

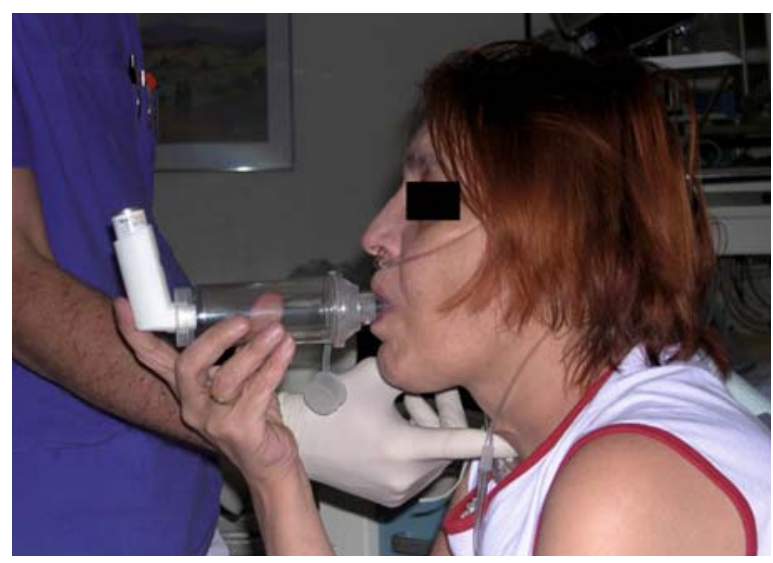

- Abb. 15.9. Tracheotomierte Patientin. Nach Entfernen der Kanüle wird das Tracheostoma mit dem Finger abgedichtet, dann wird das pMDI mit einem Hohlraumsystem inhaliert

setzt werden sollten. Über die Anwendungsdauer sollten die Vernebler überwacht werden, da diese leicht abkippen und die Medikamentenlösung ausläuft.

Tracheotomierter Patient mit Spontanatemphasen. Die Aerosoltherapie sollte möglichst in der Spontanatemphase mittels pMDI oder Vernebler verabreicht werden. Der Vernebler mit Inspirationsventil wird direkt mit der Trachealkanüle verbunden. Im Einzelfall, z. B. bei starker Verschleimung, kann die Trachealkanüle passager entfernt, das Tracheostoma mit dem Finger (• Abb. 15.9) abgedichtet und das Aerosol über den Mund appliziert werden. Der Vorteil besteht insbesondere darin, dass der Patient vor der Inhalation kräftiger abhusten kann und danach eine tiefere Inspiration ohne Impaktion an Mukusplaques in den zentralen Atemwegen erreicht werden kann.

\subsubsection{Pädiatrie}

Bei der Behandlung von Kindern sind das Alter und die Kooperationsfähigkeit von entscheidender Bedeutung bei der Auswahl eines geeigneten Inhalationssystems. 
0-2 Lebensjahre. Verwendet werden Düsen- oder Ultraschallverneblern mit einer Partikelgröße unter 2-3 $\mu \mathrm{m}$ und mit kontinuierlichem Fluss in Verbindung mit einer Nasenmaske. Wenn diese nicht dicht auf das Gesicht des Kleinkinds aufgesetzt wird oder das Kind zu schreien beginnt, geht die Lungendeposition gegen Null. Im Zweifelsfall führt der Versuch einer topischen Therapie nur $\mathrm{zu}$ einer Belastung der Eltern und des Kindes, wenn zusätzlich die Therapiedauer (z. B. 3- bis 4-mal $10 \mathrm{~min}$ ) in Betracht gezogen wird.

2-4 Lebensjahre. Vernebler, zunehmend über Mundstück oder pMDI mit für Kinder geeigneten Hohlraumsystemen (z.B. Nebuhaler ${ }^{\oplus}$, Babyhaler ${ }^{\oplus}$, Watchhaler ${ }^{\circledR}$ ) kommen zum Einsatz. Prinzipiell sind pMDI, die kleine Partikel (MMAD 1-2 $\mu \mathrm{m}$ ) liefern, notwendig (Ventolair", Junik $^{\circledR}$, Alvesco ${ }^{\circledR}$ noch ohne Zulassung). Aufgrund der kleineren Atemwegsdurchmesser und der niedrigen Inspirationsflüsse bei Kindern kann die Lungendeposition dadurch mehr als verdoppelt werden.

Ab dem 5. Lebensjahr. pMDI und DPI werden zunehmend eingesetzt. Pulversysteme kommen dann infrage, wenn die Kinder hohe Inspirationsflüsse von $301 / \mathrm{min}$ und mehr über das Gerät erreichen können, um die Partikelgröße klein zu halten.

\subsubsection{Systemische Aerosoltherapie}

Inhalatives Insulin. Im Sommer 2006 wurde Exubera ${ }^{\circ}$ bei Patienten mit Diabetes mellitus Typ I und II in Deutschland zugelassen. Aerosolgenerator ist ein Pulversystem, in das eine Kapsel mit Insulin eingelegt wird. Über einen Spannmechanismus wird das Aerosol in einen Spacer geleitet, aus dem der Patient die »stehende" Aerosolwolke inhaliert. Hinweise auf eine Lungentoxizität, die über den Spontanverlauf der Grunderkrankung hinausgehen, haben sich bisher nicht ergeben, zumal nur in jeder 15. Alveole ein Insulinmolekül deponiert wird. Es wird die 10-fache Dosis im Vergleich zur subkutanen Gabe aufgrund einer erniedrigten Bioverfügbarkeit benötigt. Die Dosiskonstanz ist höher einzuschätzen im Vergleich zur stark schwankenden subkutanen Resorption. Rauchen (höhere bronchiale Absorption) und chronische Atemwegserkrankungen stellen Kontraindikationen dar. Allerdings wurde das System aus Umsatz- und Kostengründen wieder vom Markt genommen.

Interessante Ansätze sind weiterhin die Inhalation von Heparin zur Thromboseprophylaxe und die Inhalation von Impfstoffen (z. B. Masern). Gerade in den Entwicklungsländern könnten dadurch eine große Zahl von Kindern ohne den Aufwand einer Subkutangabe mittels einer Verneblerinhalation geimpft werden; infektionsgefährdete Spritzen werden vermieden.

\section{Zusammenfassung}

Das ideale Inhalationssystem, das die Erfordernisse jedes Patienten, jedes Medikaments und jeder Erkrankung erfüllt, existiert nicht. Bei der Auswahl eines Inhalationssystems sind die Koordinationsfähigkeit des Patienten und die Höhe des Inspirationsflusses (möglichst bestimmt mithilfe einer Spirometrie) einzubeziehen.

Weiterhin ist die Patientenpräferenz zu berücksichtigen. Eine Instruktion des Patienten nur mithilfe des Beipackzettels ist ungenügend. Eine individuelle Schulung bei der Erstversorgung durch den Arzt, den Praxismitarbeiter oder den Apotheker ist ebenso unerlässlich wie die Überprüfung der Inhalationstechnik im Behandlungsverlauf.

\section{Literatur}

Boe J, Dennis JH, O`Driscoll BR (2001) European Respiratory Task Force: European Society Guidelines on the use of nebulizers. Eur Resp $J$ $18: 228-242$

Dhand R (2005) Inhalation therapy with metered-dose inhalers and dry powder inhalers in mechanically ventilated patients. Respir Care 50:1331-1334

Dolovich MB, Maclntyre NR, Anderson PJ et al. (2000) Consensus statement: Aerosols and delivery devices. J Aerosol Med 13:291-300

Everard ML, Devadason SG, LeSouef PN (1997) Flow early in the inspiratory manoeuvre affects the aerosol particle size distribution from a Turbuhaler. Respir Med 91:624-628

Haidl P, Schmidt F, Wiese C, Köhler D (2006) Peak inspiratory flow rate after methacholine challenge in asthmatic patients and its impact on the effect of formoterol via different inhalers. J Aerosol Med 19:364-371

Kohlhäufl M, Haidl P, Voshaar Th et al. (2004) Pulverinhalationssysteme. Dtsch Med Wochenschr 129:2048-2052

Köhler D, Fleischer W (2000) Theorie und Praxis der Inhalationstherapie. Arcis, München

Lenney J, Innes JA, Crompton GK (2000) Inappropriate inhaler use: assessment of use and patient preference of seven inhalation devices. Respir Med 94:496-500

Matthys H (1998) Praxis der Aerosoltherapie. In: Scheuch G (Hrsg) Aerosole in der Aerosoltherapie II. Dustri, München

O'Riordan TG, Palmer LB, Smaldone GC (1994) Aerosol deposition in mechanically ventilated patients. Am J Respir Crit Care Med 149:214-219

Patton JS, Bukar JG, Eldon MA (2004) Clinical pharmacokinetics and pharmacodynamics of inhaled insulin. Clin Pharmacokinet 43:781-801

Pauwels R, Newman S, Borgstrom L (1997) Airway deposition and airway effects of anti-asthmatic drugs delivered from metered-dose inhalers Eur Respir J 10:2127-2138

Schuepp KG, Straub D, Moller A, Wildhaber JH (2004) Deposition of aerosols in infants and children 17:153-156

Voshaar Th (2005) Therapie mit Aerosolen. UNI-MED, Bremen

Voshaar Th, App EM, Berdel D et al. (2001) Empfehlungen für die Auswahl von Inhalationssystemen zur Medikamentenverabreichung. Pneumologie 55:579-586 


\subsection{Nichtinvasive Beatmung - Therapie der Atempumpinsuffizienz}

M. Dreher, J.H. Storre, W. Windisch

\subsubsection{Grundlagen der nichtinvasiven Beatmung}

\section{Definition und Historie}

Die nichtinvasive Beatmung (»noninvasive ventilation", NIV) bezeichnet eine mechanische Ventilation der Lungen ohne Zuhilfenahme künstlicher endotrachealer Atemwege. Der Beatmungszugang, der die anatomischen Atemwege des Patienten und die künstlichen Atemwege des Respirators miteinander verbindet, liegt somit außerhalb des menschlichen Körpers. Grundsätzlich wird dabei zwischen einer Negativ- und einer Positivdruckbeatmung unterschieden.

Eine Negativdruckbeatmung mittels Unterdruckrespiratoren ist historisch älter als die Positivdruckbeatmung und ist insbesondere als »eiserne Lunge" während der Polioepidemie in der ersten Hälfte des 20. Jahrhunderts bekannt geworden (•Abb.15.10). Der große technische Aufwand, die eingeschränkte Toleranz vonseiten des Patienten sowie die nicht selten insuffiziente Ventilation im Falle eines Kollapses der oberen Atemwege haben diese Beatmungsform jedoch in den Hintergrund treten lassen.

Die Klinikroutine wird heute daher im Wesentlichen von der Positivdruckbeatmung (»noninvasive positive pressure ventilation «, NPPV) bestimmt. Diese wird vornehmlich über Gesichtsmasken durchgeführt. Eine Anwendung der NPPV findet dabei sowohl auf der Intensivstation (»intensive care«) als auch im häuslichen Bereich (»home care») statt.

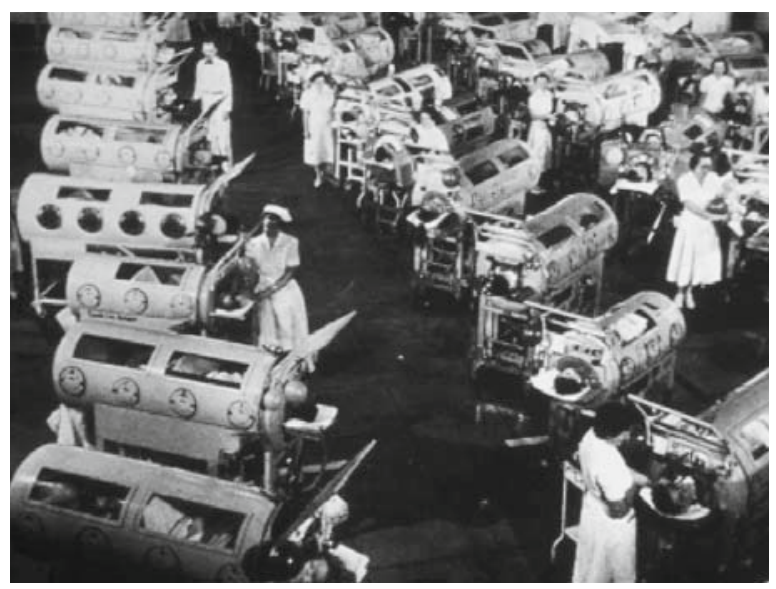

- Abb. 15.10. »ron lung ward« des Rancho Los Amigos Hospitals in Downey, Kalifornien, USA, um 1953

\section{Beatmungszugänge}

Die Beatmungszugänge bei der NPPV umschließen in der Regel die Nase und/oder den Mund und werden mittels Haltebändern am Kopf fixiert. Am häufigsten werden sogenannte Nasenmasken oder Mund-Nasen-Masken eingesetzt. Hier existiert mittlerweile eine Vielzahl industriell gefertigter Masken in unterschiedlichsten Größen, sodass für die meisten Patienten ein zufriedenstellender Beatmungszugang auch für die Langzeitanwendung gefunden werden kann. Im Bedarfsfall können die Masken jedoch auch individuell angefertigt werden, um die Beatmungsqualität zu optimieren.

Die Beatmungsmasken sollten möglichst dicht aufsitzen, um ein Entweichen der Luft (Leckagen) zu vermeiden. Dabei ist jedoch darauf zu achten, dass die Masken nicht zu fest auf dem Gesicht befestigt werden, da es insbesondere im Bereich des Nasenrückens zu Druckstellen bis hin zu Ulzerationen kommen kann, die eine Fortsetzung der NPPV unmöglich machen (• Abb. 15.11). Die Auswahl der richtigen Beatmungsmaske ist essenziell für das Gelingen der Therapie und die Compliance der Patienten.

Nasenmasken. Nasenmasken umschließen lediglich die Nase (-Abb. 15.12). Der Patient sollte in der Lage sein, den Mund geschlossen zu halten, um Leckagen über den Mund zu vermeiden. Nicht selten kann jedoch ein geöffneter Mund in Bezug auf Leckagen tolerabel sein, wenn der weiche Gaumen den oralen Luftweg weitgehend
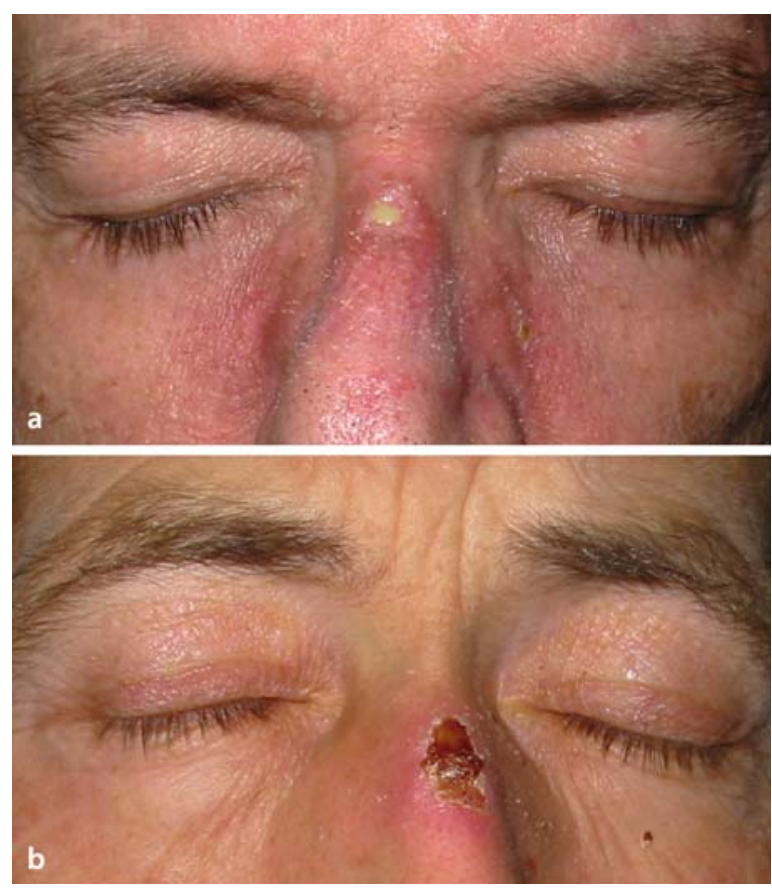

- Abb. 15.11a,b. Druckschäden im Bereich der Nasenwurzel bedingt durch eine Gesichtsmaske. a Offene Ulzeration, eitrig belegt. b Abheilung mit Krustenbildung 
verschließt. Nasenmasken sind der dominierende Beatmungszugang bei der stabilen chronischen Atempumpinsuffizienz im Rahmen einer Heimbeatmungstherapie. Hierunter ist am leichtesten eine Kommunikation möglich und bei zunehmender Beatmungspflicht selbst eine Nahrungsaufnahme nicht ausgeschlossen. Weiterhin sind die Patienten in der Lage, unter der Beatmung Sputum abzuhusten. Die am häufigsten eingesetzten Nasenmasken umschließen die Nase vollständig. Es existieren jedoch auch Nasenmasken, die den Nasenlöchern aufliegen oder leicht in sie hineinragen (Nasenpillows). Diese kleineren Beatmungsmasken verhindern Druckstellen auf dem Nasenrücken, sind aber in der Regel bei höheren Inspirationsdrücken nicht geeignet.
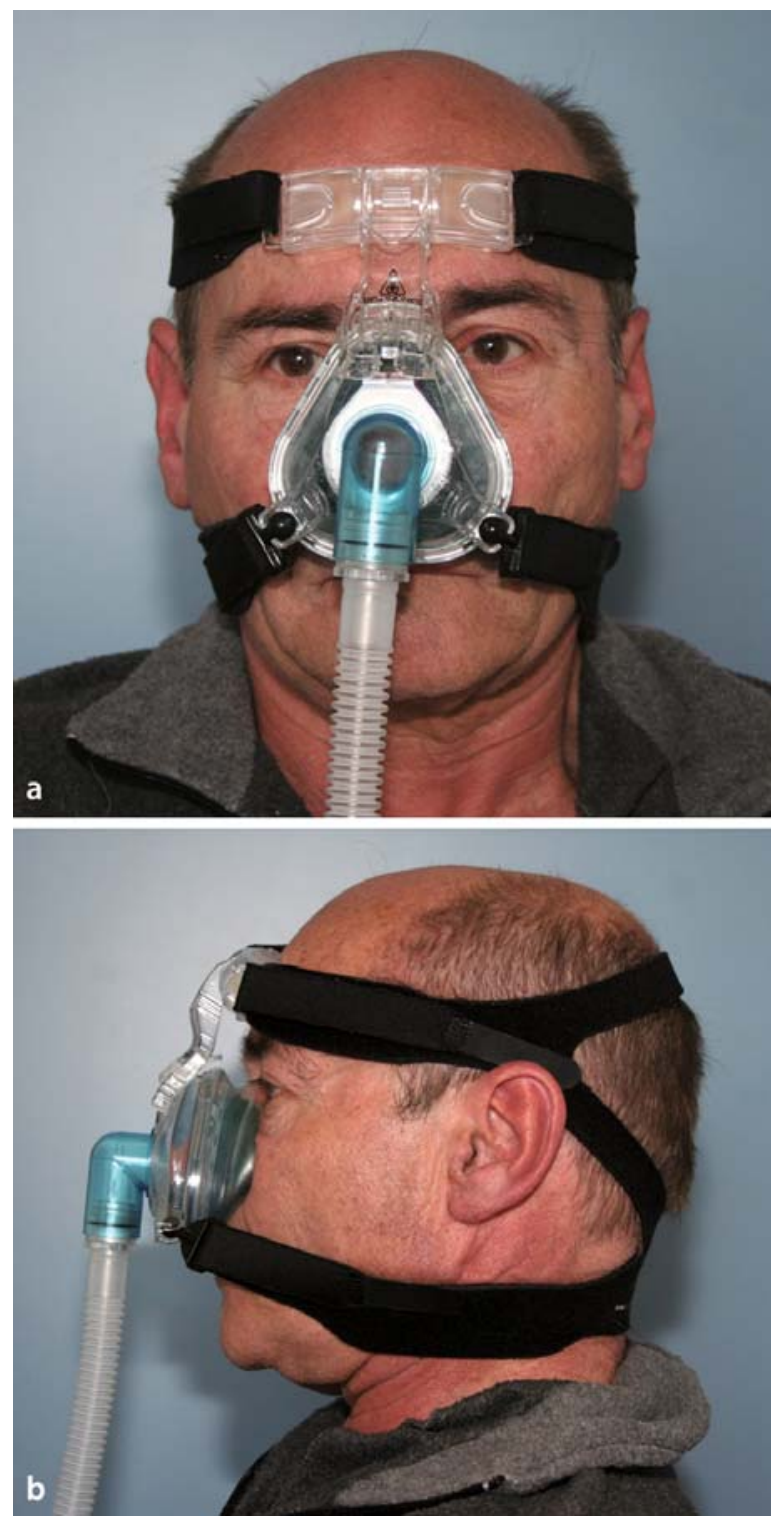

- Abb. 15.12a,b. COPD-Patient mit Nasenmaske
Mund-Nasen-Masken. Mund-Nasen-Masken umschließen zusätzlich zur Nase auch den Mund (- Abb. 15.13). Sie finden ihre Anwendung bei Patienten, die überwiegend durch den Mund atmen oder nicht in der Lage sind, den Mund unter einer Beatmung mit einer Nasenmaske geschlossen zu halten. Da dieses Problem überwiegend bei akuter respiratorischer Insuffizienz auftritt, sind die Mund-Nasen-Masken der dominierende Beatmungszugang bei der Akutanwendung der NPPV auf der Intensivstation.
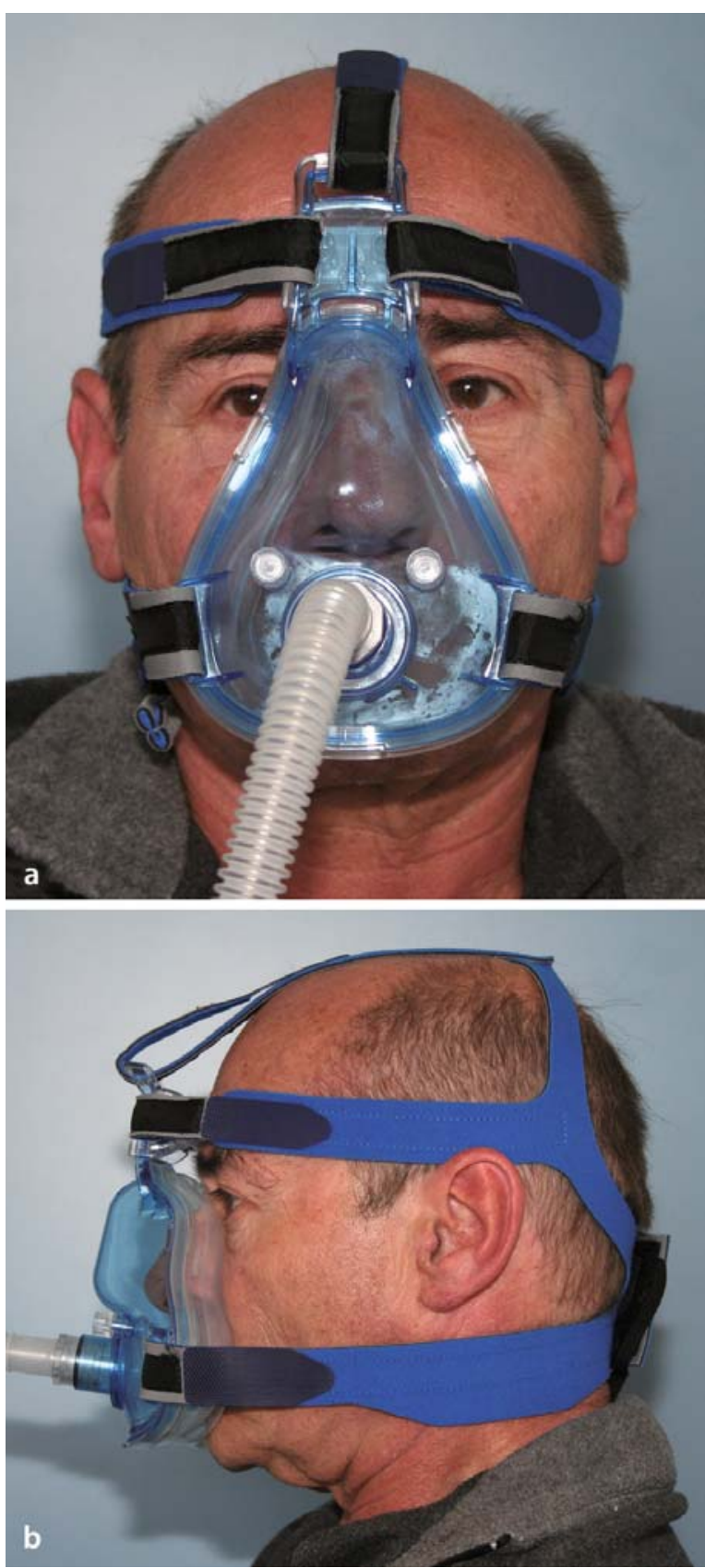

- Abb. 15.13a,b. COPD-Patient mit Mund-Nasen-Maske 
Individuelle Masken. Individuelle Masken (• Abb. 15.14) werden bei Patienten benötigt, die langzeitbeatmet werden und die konventionellen Beatmungsmasken nicht tolerieren. Die häufigste Indikation zur Anpassung einer
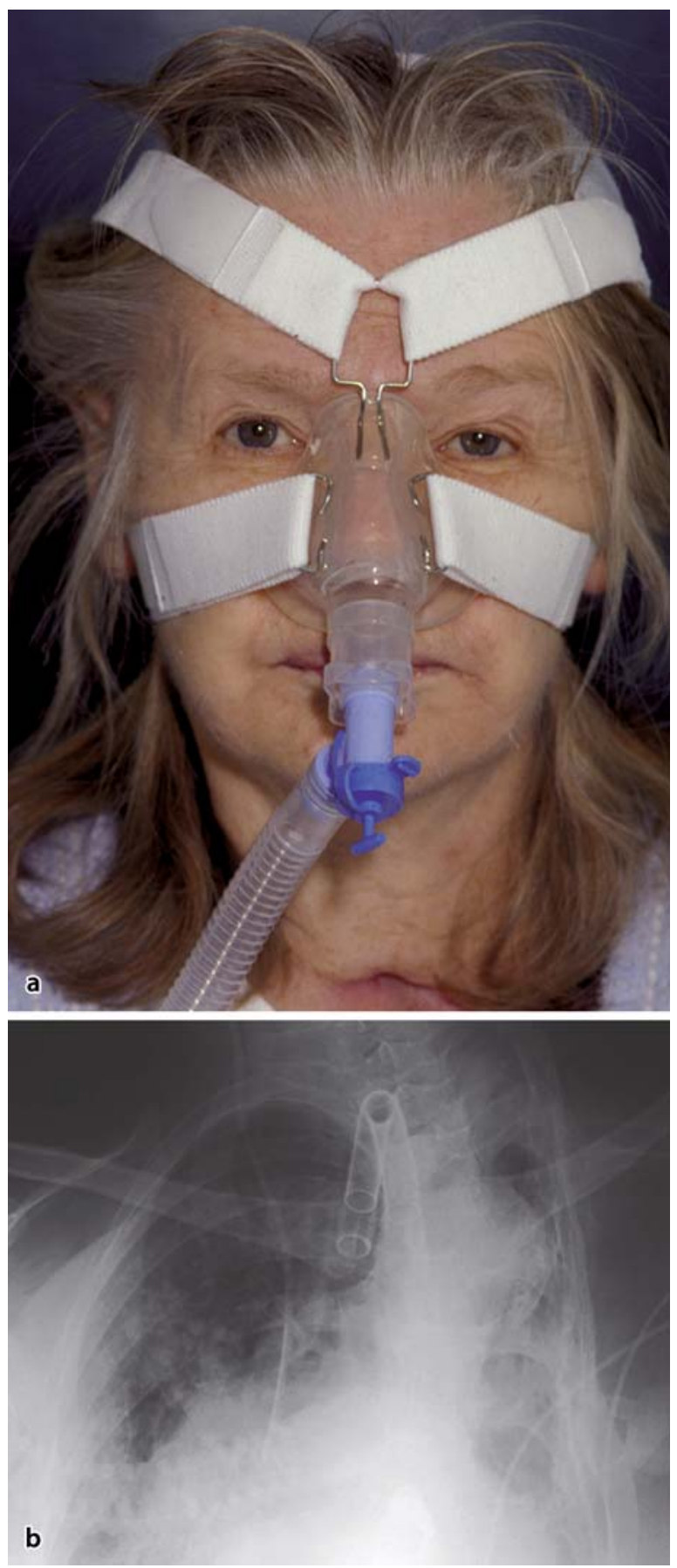

- Abb.15.14a,b. Patientin mit posttuberkulösem Syndrom. a Die häusliche nichtinvasive Beatmung (NIV) über eine individuell angefertigte Nasenmaske erfolgte nach kompliziertem Weaning von der invasiven Beatmung, die im Rahmen einer akuten respiratorischen Insuffizienz infolge Pneumonie notwendig wurde.b Die Residuen nach Tracheotomie sind bei der Patientin noch zu erkennen individuellen Maske sind Druckstellen durch konventionelle Masken (• Abb. 15.11). Die Anpassung einer individuellen Maske ist kosten- und zeitintensiv, berücksichtigt jedoch die individuelle Gesichtsanatomie des Patienten und kann durch eine angenehmere Passform zu einer höheren Akzeptanz der Therapie führen. Häufig ist die Verfügbarkeit der individuellen Masken nicht überall und zu jeder Zeit gegeben, sodass die Verwendung industrieller Masken primär zu empfehlen ist. Durch die heutige Maskenvielfalt und die große Zahl verschiedener Maskenanbieter gelingt es fast immer, für einen Patienten eine passende Maske zu finden. Erwähnt sei aber auch, dass eine Veränderung der Gesichtskonfiguration, z. B. bei Gewichtsabnahme oder im Zuge von Operationen, die Notwendigkeit zum Wechsel des Beatmungszugangs nach sich ziehen kann.

Seltenere Beatmungszugänge. Seltener eingesetzte Beatmungszugänge sind Vollgesichtsmasken, die das gesamte Gesicht inklusive der Augen umschließen. Vollgesichtsmasken bieten den Vorteil, dass sie nicht direkt auf dem Nasenrücken aufliegen. Somit führen sie zu einer Entlastung bei dort aufgetretenen Durckstellen. Ihre Transparenz ermöglicht zusätzlich eine Vergrößerung des Gesichtsfelds. Daneben existieren noch Beatmungshelme, die den gesamten Kopf umschließen und am Hals abdichten. Je größer die Beatmungszugänge sind, desto größer ist auch das Totraumvolumen und somit die Gefahr der verminderten $\mathrm{CO}_{2}$-Elimination. Der Einsatz der Beatmungshelme ist deshalb nach der aktuellen Studienlage nicht bei einer Hyperkapnie zu empfehlen, konnte jedoch erfolgreich bei akuter hypoxämischer respiratorischer Insuffizienz angewendet werden.

\section{Nebenwirkungen einer NPPV}

Die wesentlichen Nebenwirkungen einer NPPV sind in - Tab. 15.2 dargestellt. Grundsätzlich sind Nebenwirkungen sehr häufig, jedoch in den meisten Fällen ungefährlich. Einige Nebenwirkungen sind jedoch zum Teil sehr lästig für den Patienten und können gelegentlich zum Therapieabbruch führen, weshalb Nebenwirkungen soweit wie möglich minimiert werden müssen. Entsprechend sind in - Tab. 15.2 Lösungsstrategien vermerkt. Es sei aber an dieser Stelle darauf hingewiesen, dass es für die Probleme bei der NPPV keine gültigen Patentlösungen gibt und nicht selten sehr individuelle Lösungsstrategien gefunden werden müssen. Die Erfahrung mit der NPPV sowohl vonseiten des Therapeuten als auch vonseiten des Patienten ist hier eine wesentliche Voraussetzung.

Das wesentliche Problem bei der NPPV stellt die insuffiziente Ventilation dar. Intoleranz, Leckagen und Obstruktionen der oberen Atemwege bilden die wesent- 
- Tab. 15.2. Häufigkeit ${ }^{\mathrm{a}}$ von Nebenwirkungen und Komplikationen bei der NPPV und mögliche Lösungsstrategien

\begin{tabular}{|c|c|c|}
\hline & Häufigkeit (\%) ${ }^{\mathrm{a}}$ & Mögliche Lösungsstrategie \\
\hline \multicolumn{3}{|l|}{ Maskenbezogen } \\
\hline Diskomfort & $30-50$ & Passform überprüfen, Halteband justieren, Maskentyp wechseln \\
\hline Hautreizungen im Gesicht & $20-34$ & Wundverbandplatte als Unterlage, Halteband lockern \\
\hline Klaustrophobie & $5-10$ & Kleinere Maske, schrittweise Gewöhnung \\
\hline Nasenrückenulkus & $5-10$ & Halteband lockern, neue Maske, Comfeel-Platte als Unterlage \\
\hline \multicolumn{3}{|l|}{ Luftdruck-/atemflussbezogen } \\
\hline Verstopfte Nase & $20-50$ & Topische Steroide, abschwellende Nasentropfen \\
\hline Schmerzen (Nasennebenhöhlen, Ohr) & $10-30$ & Reduktion der Beatmungsdrücke \\
\hline Trockene Schleimhäute & $10-20$ & $\begin{array}{l}\text { Meersalzspray, feuchte Nasensalbe, Mundleckage vermeiden, } \\
\text { Befeuchter (passiv/aktiv) }\end{array}$ \\
\hline Irritationskonjunktivitis & $10-20$ & Maskensitz überprüfen, Leckage beheben \\
\hline Abdominelle Blähungen & $5-10$ & Reduktion der Beatmungsdrücke, Karminativa \\
\hline Leckagen & $80-100$ & $\begin{array}{l}\text { Reduktion der Beatmungsdrücke, bei Mundleckage: Kinnband } \\
\text { oder Mund-Nasen-Maske }\end{array}$ \\
\hline \multicolumn{3}{|l|}{ Schwere Komplikationen } \\
\hline Aspirationspneumonie & $<5$ & Präventiv: vorsichtige Patientenselektion \\
\hline Hypotension & $<5$ & Reduktion der Beatmungsdrücke \\
\hline Pneumothorax & $<5$ & $\begin{array}{l}\text { Ventilation unterbrechen, Reduktion der Beatmungsdrücke, ggf. } \\
\text { Thoraxdrainage }\end{array}$ \\
\hline
\end{tabular}

${ }^{\mathrm{a} G e s c h a ̈ t z t e ~ H a ̈ u f i g k e i t ~ b a s i e r e n d ~ a u f ~ m e h r e r e n ~ S t u d i e n ~ n a c h ~ M e h t a ~(2001) ~}$

lichen Gründe hierfür. Hinzu kommt, dass die Atemwege nicht geblockt sind. Schwere Aspirationen oder auch Pneumothoraces sind aber seltene Komplikationen. Bei der NPPV kommt es außerdem zu einer Erhöhung des intrathorakalen Drucks. Dies bedingt eine Zunahme der rechtsventrikulären Nachlast sowie eine Abnahme der linksventrikulären Vor- und Nachlast. Dies kann zu einem Blutdruckabfall führen, insbesondere bei Akutanwendung der NPPV bei hypovolämischen Patienten, z. B. solchen, die in der Zeit zuvor wenig getrunken haben. Die Abnahme der linksventrikulären Vor- und Nachlast kann jedoch beim kardialen Lungenödem auch therapeutisch genutzt werden, da in dieser Situation durch die Verbesserung der linksventrikulären Pumpleistung das Herzminutenvolumen verbessert werden kann.

\section{Respiratoren}

Für die NPPV steht eine Vielzahl verschiedener Beatmungsgeräte zur Verfügung. Prinzipiell kann man zwischen sogenannten Heimbeatmungsgeräten (»home care«) und Beatmungsgeräten auf der Intensivstation (»critical care») unterscheiden. Die Heimbeatmungsgeräte sind im Vergleich zu Intensivbeatmungsgeräten deutlich kleiner und kostengünstiger. Sie sind von externer Stromzufuhr, nicht jedoch von einem Druckluftanschluss abhängig. Dadurch sind diese Respiratoren mobil einsetzbar und somit sowohl für die häusliche Beatmung als auch z. B. für Urlaubsreisen geeignet. Sie sind jedoch hinsichtlich der Beatmungsmodi und der Alarmfunktion im Vergleich zu Respiratoren auf der Intensivstation limitiert.

Intensivmedizinische Respiratoren sind in vielen Fällen von einem Druckluftanschluss abhängig. Sie wurden primär für die invasive Beatmung über Beatmungstuben entwickelt. Die neueren Generationen von Intensivrespiratoren integrieren jedoch zunehmend eigene Beatmungsmodi auch für die NPPV, wobei die speziellen Probleme der NPPV hinsichtlich Leckagekompensation und Triggerung berücksichtigt werden. 


\section{Weiteres Equipment}

Schlauchsysteme (Einschlauch- vs. Doppelschlauchsystem). In der Heimbeatmung werden primär Geräte mit Einschlauchsystemen verwendet. Die Toleranz des berechneten inspirierten Tidalvolumens liegt bei $\pm 20 \%$. Doppelschlauchsysteme werden in erster Linie in der invasiven Beatmung verwendet. Sie ermöglichen eine Messung des exspiratorischen Tidalvolumens.

Aktive und passive Befeuchtung. Die oberen Atemwege sind für die Erwärmung und Befeuchtung der Atemluft zuständig. Bei der NIV kann es trotz intakter Schleimhäute zur Austrocknung kommen. Abhilfe schaffen aktive bzw. passive Befeuchtersysteme. Aktive Befeuchter arbeiten thermostatgesteuert. Sie erwärmen Wasser in einer Befeuchterkammer und reichern die Inspirationsluft mit Feuchtigkeit an. Passive Befeuchter sind sogenannte Wärme- und Feuchtigkeitsaustauscher (»heat and moisture exchanger «, HME). Sie speichern die exspirierte Wärme und Feuchtigkeit in einem Filter und geben sie wieder an die Inspirationsluft ab. Die maximale Verwendungsdauer beträgt $24 \mathrm{~h}$, da es sonst zu erhöhten Filterwiderständen und zu einer $\mathrm{CO}_{2}$-Retention kommen kann.

Ausatemventile. Es gibt 2 Möglichkeiten, Kohlendioxid aus der Exspirationsluft zu eliminieren. Aktiv gesteuerte Exspirationsventile transportieren Kohlendioxid über eine Membran nach außen. Passiv gesteuerte Exspirationsventile eliminieren Kohlendioxid über eine permanente definierte Leckage. Der gleiche Effekt kann durch eine Beatmungsmaske mit Leckageöffnungen erreicht werden. Manche Geräte bieten beide Möglichkeiten.

Sauerstoffzufuhr. Die Zufuhr von Sauerstoff während der Beatmung kann durch externe oder interne Sauerstoffadapter gewährleistet werden. Bei der internen $\mathrm{Zu}$ fuhr wird das zusätzliche Volumen durch den Sauerstoff für die Berechnung des Tidalvolumens mit berücksichtigt. Dies ist bei geringen Atemzugvolumina und hohem Sauerstoffbedarf, z. B. bei der Beatmung von Kindern, von Vorteil.

Bakterienfilter. Bakterienfilter verhindern die Kontamination von Heimbeatmungsgeräten und schützen so vor allem immunsupprimierte Patienten vor einer Infektion. Wird ein Heimbeatmungsgerät stationär bei mehreren Patienten eingesetzt, ist die Verwendung eines Bakterienfilters obligat.

\section{Beatmungsverfahren}

Aufgrund einer Vielzahl verschiedener Beatmungsgeräte und Hersteller werden die vewendeten Beatmungsmodi und deren Abkürzungen immer unübersichtlicher. Einen
Überblick über die gängigen Beatmungsmodi und einige weitere nützliche Abkürzungen gibt -Tab.15.3. Grundsätzlich kann bei der NPPV primär mit Volumen- oder Druckvorgabe ventiliert werden. Bei der volumenkontrollierten Beatmung (»volume controlled ventilation«, VCV) liefert der Respirator ein definiert am Respirator eingestelltes Atemzugvolumen, wobei der Inspirationsdruck in Abhängigkeit von Resistance, Compliance und Leckagen variabel ist. Dagegen wird bei der druckkontrollierten Beatmung ("pressure controlled ventilation", PCV) bei jedem Atemzug ein definiert am Respirator eingestellter Inspirationsdruck bereitgestellt, bei dem das Atemzugvolumen entspechend variiert.

Der Vorteil einer VCV liegt darin, dass ein definiertes Atemzugvolumen sichergestellt werden kann. Allerdings können dabei sehr hohe Beatmungsdrücke notwendig werden, die von den Patienten oft als unangenehm empfunden werden. Wenn die vom Respirator gelieferte Luft nicht nur über den Luftweg in die Lungen, sondern auch über die Speiseröhre in den Magen gelangt, kann es zu gastrointestinalen Nebenwirkungen kommen. Ein weiterer Nachteil der VCV liegt in der fehlenden Möglichkeit, Leckagen zu kompensieren.

Bei Leckagen besitzt die PCV den Vorteil einer Kompensation, da der durch eine Leckage entstandene Druckverlust zu einer Erhöhung des Inspirationsflusses und damit des applizierten Volumens des Ventilators führt. Bei erhöhten Atemwegswiderständen kann unter der PCV der eingestellte Inspirationsdruck jedoch auch erreicht sein, ohne dass ein suffizientes Atemzugvolumen bereitgestellt wurde.

Anfang der 1990er-Jahre waren fast ausschließlich Respiratoren mit Volumenvorgabe im Bereich der Heimbeatmung im Einsatz, wohingegen heutzutage der Anteil der NPPV mittels Druckvorgabe dominiert. Vergleichende Studien der VCV und PCV konnten keine wesentlichen Unterschiede in Hinsicht auf die Verbesserung des Gasautauschs oder der Schlafqualität aufdecken, jedoch zeigten sich Vorteile für die PCV durch eine bessere Toleranz vonseiten der Patienten. Neuere Beatmungsmodi besitzen die Möglichkeit, die Vorteile der volumen- und der druckkontrollierten Beatmung in einem Modus zu kombinieren. Dabei wird mit Druckvorgabe ventiliert und gleichzeitig ein Zielvolumen definiert, das der Respirator im Rahmen vorher eingestellter Grenzen für den Inspirationsdruck versucht zu erreichen.

Bei einer NPPV kann die Atemarbeit in Analogie zur invasiven Beatmung komplett vom Respirator (kontrollierte Beatmung) oder auch nur teilweise übernommen werden. Bei letzterer Beatmungsform ist der Patient in der Lage, nach Einstellung einer Triggerschwelle den Beginn der Inspiration selber auszulösen, erfährt jedoch eine inspiratorische Unterstüzung durch den Respirator 
- Tab. 15.3. Beatmungsmodi und gebräuchliche Abkürzungen

\begin{tabular}{|c|c|}
\hline ASB & $\begin{array}{l}\text { Druckunterstützte Spontanatmung (»assisted } \\
\text { spontaneous breathing«) }\end{array}$ \\
\hline ASV & Adaptive Servoventilation (»adaptive servo ventilation«) \\
\hline ASPCV & $\begin{array}{l}\text { Assistiert-druckkontrollierte Beatmung (»assisted- } \\
\text { pressure controlled ventilation«) }\end{array}$ \\
\hline BiPAP $^{\infty}$ & $\begin{array}{l}\text { Biphasischer positiver Atemwegsdruck (»biphasic } \\
\text { positive airway pressure«) }\end{array}$ \\
\hline CMV & "Controlled mandatory ventilation« \\
\hline CPAP & $\begin{array}{l}\text { Kontinuierlicher positiver Atemwegsdruck } \\
\text { (»continuous positive airway pressure») }\end{array}$ \\
\hline $\mathrm{FiO}_{2}$ & Inspiratorische Sauerstofffraktion \\
\hline HFOV & "High frequency oscillatory ventilation« \\
\hline IPPV & $\begin{array}{l}\text { Intermittierende Beatmung mit positivem Druck } \\
\text { (»intermittend positive pressure ventilation«) }\end{array}$ \\
\hline ISB & Intermittierende Selbstbeatmung \\
\hline NIV & Nichtinvasive Beatmung (»non-invasive ventilation«) \\
\hline NPPV & $\begin{array}{l}\text { Nichtinvasive Beatmung mit positivem Druck } \\
\text { (»non-invasive positive pressure ventilation«) }\end{array}$ \\
\hline PAV & $\begin{array}{l}\text { Proportionale assistierte Beatmung (»proportional } \\
\text { assist ventilation«) }\end{array}$ \\
\hline PCV & $\begin{array}{l}\text { Druckkontrollierte Beatmung (»pressure controlled } \\
\text { ventilation«) }\end{array}$ \\
\hline PEEP & $\begin{array}{l}\text { Positiver endexspiratorischer Druck (»positive } \\
\text { endexspiratory pressure«) }\end{array}$ \\
\hline PSV & $\begin{array}{l}\text { Druckunterstützte Spontanatmung (»pressure } \\
\text { support ventilation«) }\end{array}$ \\
\hline SIMV & "Synchronized intermittend mandatory ventilation" \\
\hline VCV & $\begin{array}{l}\text { Volumenkontrollierte Beatmung (»volume controlled } \\
\text { ventilation«) }\end{array}$ \\
\hline
\end{tabular}

während der Inspiration (assistierte Beatmung). Zusätzlich kann der Patient die Inspirationsphase terminieren und die Exspiration einleiten (z. B. bei "pressure support ventilation«), wobei die Triggerschwelle für die Einleitung der Exspiration bei einigen Respiratoren fest vorgegeben ist, bei anderen aber ebenfalls am Respirator eingestellt werden kann.

Nicht selten besteht fälschlicherweise die Annahme, eine kontrollierte Beatmung sei nur bei einer suffizienten Sedation des Patienten möglich. Das ist jedoch nicht richtig. Auch wenn Patienten mit uneingeschränkter ventilatorischer Kapazität sich häufig nur schwer auf einen vorgegebenen Atemrhythmus einstellen lassen, kann bei Patienten mit Atempumpinsuffizienz und Hyperkapnie eine kontrollierte Beatmung durchführbar und auch sinnvoll sein, da hierdurch eine überanspruchte und erschöpfte Atemmusklatur maximal entlastet werden kann. Vorsicht ist jedoch bei Patienten mit akuter respiratorischer Insuffizienz geboten, da hier initial zu schnell hochtitrierte Beatmungsdrücke und Atemfrequenzen zu einer Intoleranz der NPPV oder im Falle einer akut exazerbierten COPD auch zu einer dynamischen Lungenüberblähung des Patienten führen können.

\section{Indikationen}

Mit Beginn der 80er-Jahre des letzten Jahrhunderts wurde die NPPV zunehmend im Rahmen einer häuslichen Beatmung eingesetzt. Prinzipiell können alle Erkrankungen, die zu einer chronischen ventilatorischen Insuffizienz führen, eine Indikation für eine häusliche Beatmung darstellen. Zahlenmäßig dominieren hier Patienten mit neuromuskulären Erkrankungen wie der Muskeldystrophie Duchenne oder der amyotrophen Lateralsklerose, Patienten mit Thoraxdeformitäten wie bei den verschiedenen Formen der Kyphoskoliose, Patienten mit COPD sowie Patienten mit Adipositas-Hypoventilationssyndrom. Eine Übersicht und weiterführende Darstellung über die verschiedenen Erkrankungen, die zu einer chronischen Insuffizienz der Atempumpe führen, findet sich im $>$ Kap. 2.2.

Die NPPV hat jedoch seit Beginn der 90er-Jahre des letzten Jahrhunderts auch zunehmend Einzug in die Inensivstationen erhalten, wo sie auch bei akuter respiratorischer Insuffizienz eingesetzt wird. Ein wesentlicher Indikationsbereich besteht für die akut auftretenden Atempumpstörungen mit Hyperkapnie und respiratorischer Azidose. Die akut exazerbierte COPD stellt hier ein Paradebeispiel dar. Die NPPV kann jedoch auch bei primären Gasaustauchstörungen eingesetzt werden, die initial keine Hyperkapnie präsentieren. Die folgenden Kapitel stellen den Anwedungsbereich bei den unterschiedlichen Erkrankungen sowohl für die akute als auch für die chronische respiratorische Insuffizienz dar.

\subsubsection{Nichtinvasive Beatmung bei akuter respiratorischer Insuffizienz}

\section{Invasive und nichtinvasive Beatmung}

Pathophysiologisch können 2 Formen der akuten respiratorischen Insuffizienz (ARI) unterschieden werden. Akute pulmonale Erkrankungen, wie z.B. eine schwere Pneumonie, führen primär aufgrund ihrer führenden Gasaustauschstörung zu einer hypoxämischen ARI mit respiratorischer Partialinsuffizienz. Auf der anderen Seite führen ventilatorische Störungen, z. B. durch eine Erschöpfung der Atemmuskulatur, zu einer hyperkapnischen ARI mit respiratorischer Globalinsuffizienz. 
Die wesentliche Rationale der NPPV im Rahmen einer akuten respiratorischen Insuffizienz stellt die Vermeidung einer Intubation bzw. invasiven Beatmung dar. Hierdurch können die Nebenwirkungen einer invasiven Beatmung, wie z. B. die tubusassoziierte Pneumonie, vermieden werden. Zahlreiche Studien konnten belegen, dass dadurch die Intensivstations- und Krankenhausaufenthaltsdauer reduziert sowie die Mortalität verbessert werden konnten. Die wesentlichen Vorteile der NPPV gegenüber der invasiven Beatmung sind in der Übersicht zusammengestellt.

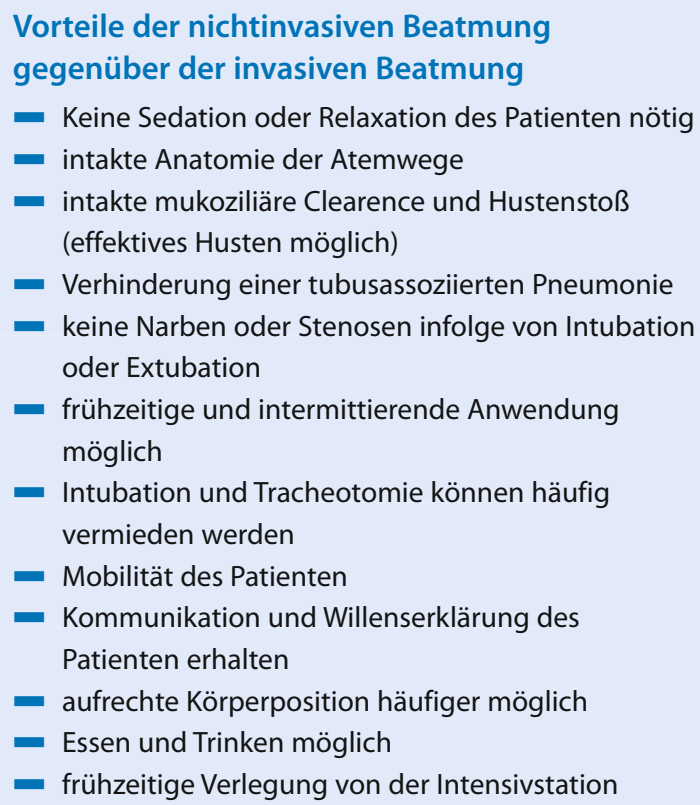

Selbstverständlich besitzt die invasive Beatmung gegenüber der NPPV die Vorteile der Vermeidung von Leckagen und des sicheren, geblockten Atemwegszugangs mit daraus resultierender initial effektiveren Beatmung. Aus diesem Grund hat die invasive Beatmung bei schwerster ARI mit vitaler Bedrohung des Patienten weiterhin ihren festen Stellenwert. Somit muss immer abgewogen werden, ob ein nichtinvasiver Beatmungsversuch möglich oder ob eine lebensrettende Intubation unumgänglich ist.

In diesem Zusammenhang sei erwähnt, dass eine NPPV eine notwendige und unumgängliche Intubation nicht unötig hinauszögern darf. So sollte in den ersten 1-2 $\mathrm{h}$ der Akutanwendung einer NPPV der Therapieerfolg abgeschätzt werden. Kommt es in diesem Zeitraum zu einer Verbesserung der Symptome und der Blutgase, kann mit großer Wahrscheinlichkeit die Intubation vermieden werden, obwohl auch sogenannte Spätversager nach Tagen der primär erfolgreichen NPPV beschrieben sind. Kann in diesem Zeitraum jedoch keine Verbesserung der respiratorischen Situation erzielt werden, ist das
Risiko für ein Scheitern der NPPV hoch, entsprechend sollte die Indikation zur Intubation erwogen werden.

NPPV und invasive Beatmung sind somit unterschiedliche Therapieverfahren, die sich gegenseitig ergänzen und deren Indikation in Abhängigkeit von der Schwere der Erkrankung und der Erfahrung des Teams auf der Intensivstation gestellt werden sollte. Ebenso muss auch der zu erwartende Krankheitsverlauf in die Therapieentscheidung mit einbezogen werden. So ist z. B. verständlich, dass ein Patient mit schwerer Sepsis, ARDS und zu erwartendem langwierigem und schwierigem Beatmungsmanagement kein geeigneter Kandidat für eine NPPV ist.

(?)

Invasive und nichtinvasive Beatmung sind keine konkurrierenden, sondern sich ergänzende Therapiestrategien.

Für den Anwender der NPPV ist es wichtig, die Vorteile der NPPV, aber auch ihre Grenzen eindeutig zu verstehen. Diesbezüglich müssen auch die Kontraindikationen für eine NPPV bedacht werden. Früher wurde zwischen absoluter und relativer Kontraindikation unterschieden. Eine absolute Kontraindikation war z. B. ein bewusstseinseingeschränkter Patient. Auch hier gibt es mittlerweile Arbeiten, die gezeigt haben, dass der Einsatz der NIV bei komatösen Patienten im Rahmen einer hyperkapnischen Enzephalopathie möglich und mit Erfolg durchführbar ist. Die meisten Kontraindikationen sind somit relativ. Die Grenze zwischen Indikation und Kontraindikation ist entsprechend stark abhängig von der Schwere der Erkrankung sowie von der Erfahrung des behandelnden Personals und der medizinischen Infrastruktur. So sei auch erwähnt, dass eine NPPV in Kombination mit intermittierendem bronchoskopischem Absaugen auch bei Patienten mit ausgeprägter Hypersekretion und eitriger Sekretbildung möglich ist. In der folgenden Übersicht wird daher keine Unterscheidung zwischen relativer und absoluter Kontraindikation getroffen.

\section{Kontraindikationen für eine NIV}

- Komatöser Patient

- Schnappatmung, fehlende Spontanatmung oder Apnoe

- massive Agitation und/oder notwendige Analgosedierung

- Hypersekretion

- Verlegung der Atemwege

- schwere Azidose $(\mathrm{pH}<7,1)$

- Gesichtstrauma

- Ileus

- gastrointestinale Blutungen, Hämoptoe oder Nasenbluten

- Maskenintoleranz

- hämodynamisch instabile Patienten 


\section{Therapie der hyperkapnischen akuten respiratorischen Insuffizienz}

Die erschöpfte Atempumpe ist pathophysiologisch die Grundlage für eine hyperkapnische ARI. Dabei kommt es immer zu einem Ungleichgewicht zwischen der muskulären Belastung und der muskulären Kapazität. Entsprechend ist in Abhängigkeit von der Grunderkrankung die zu leistende Atemarbeit erhöht oder die atemmuskuläre Kraft erniedrigt. Häufig findet sich sogar eine Kombination beider Komponenten. Alle Erkrankungen, die zu einer gestörten Atempumpfunktion führen ( $\triangleright$ Kap. 2.2), können auch mit einer akuten Verschlechterung der respiratorischen Situation einhergehen. Kennzeichnend ist dann die Hyperkapnie $\left(\mathrm{P}_{\mathrm{a}} \mathrm{CO}_{2}>45 \mathrm{mmHg}\right)$ sowie die respiratorische Azidose $(\mathrm{pH}<7,35)$. Auslöser für eine akute Verschlechterung der Ventilation sind häufig respiratorische Infekte auf dem Boden einer vorbestehenden chronischen ventilatorischen Insuffizienz.

Die wichtigste Erkrankung stellt die akut exazerbierte COPD dar. Bei der Behandlung dieser Erkrankung werden durch die NPPV im Wesentlichen 2 Ziele verfolgt: Die Reduktion der Atemarbeit und die Erhöhung des Tidalvolumens mit Verbesserung der alveolären Ventilation und konsekutiver Abnahme der Hyperkapnie. Da durch die zusätzliche dynamische Überblähung im Rahmen der Exazerbation (intrinsischer PEEP) die Atemarbeit deutlich gesteigert ist, kann diese durch Überwindung des intrinsischen PEEP wieder reduziert werden. Dies gelingt durch die Anwendung eines externen PEEP, der am Respirator eingestellt wird und der die kollabierenden Atemwege offen halten kann. Eine inspiratorische Druckunterstützung (z. B. durch PSV) kann dann zusätzlich die alveoläre Ventilation verbessern.

Es ist allerdings darauf zu achten, dass durch die Anwendung der NPPV die dynamische Überblähung nicht noch aggraviert wird. Daher sollte die Exspirationszeit nicht zu kurz gewählt sein und die inspiratorische Druckunterstützung in der Akutsituation nicht zu schnell hoch titriert werden. Dadurch kann der Druck des unteren Ösophagussphinkters überwunden werden, was zu vermehrtem Eintritt von Luft in den Magen führt. Dies kann dann ein weiteres Hindernis für die Atemexkursion darstellen. Immer mehr Luft gelangt in den Magen und steht dadurch nicht mehr der alveolären Ventilation zur Verfügung. Inakzeptanz und schließlich Scheitern der NPPV sind dann die logische Konsequenz.

Eine Vielzahl von großen Studien konnte darlegen, dass die NPPV in Kombination mit der Standardtherapie (Sauerstoffgabe, medikamentöse Therapie) im Vergleich zur alleinigen Standardtherapie in der Lage ist, bereits in der ersten Stunde der Anwendung die Blutgase zu verbessern und die Atemfrequenz zu senken. Dadurch konnte die Intubationsfrequenz deutlich gesenkt werden. Dies wiederum hatte eine Reduktion der tubusassoziierten Komplikationen, insbesondere die der Pneumonie, zur Folge. Schließlich wurden damit die Krankenhausaufenthaltsdauer und die Mortalität signifikant reduziert. Unter der Beachtung von Kontraindikationen ist daher der Einsatz der NPPV bei akut exazerbierter hyperkapnischer COPD mit hohem Evidenzgrad zu empfehlen.

Wichtig ist, dass die NPPV bereits frühzeitig indiziert wird ( $\mathrm{pH}=7,30-7,35)$, also zu einem Zeitpunkt, bei dem eine Intubation noch nicht angezeigt ist. Dadurch kann die Intubation am wirkungsvollsten vermieden werden. Im Gegensatz dazu ist bei Patienten mit schwerer respiratorischer Azidose $(\mathrm{pH}<7,30)$ die Einleitung einer NPPV schwieriger und die sofortige artifizielle Ventilation dringlicher. Aus diesem Grund muss bei diesen Patienten nicht selten intubiert werden. Dies unterstreicht das Konzept der sich ergänzenden Therapiestrategien in Bezug auf die Anwendung der invasiven und nichtinvasiven Beatmung bei der akut exazerbierten COPD mit hyperkapnischer ARI.

\section{Therapie der hypoxämischen akuten respiratorischen Insuffizienz}

Das Ziel der NPPV bei primär hypoxämischer akuter respiratorischer Insuffizienz besteht in der Normalisierung oder zumindest teilweisen Erhöhung einer pathologisch reduzierten funktionellen Residualkapazität. Dabei kann durch die Anwendung eines positiven Atemwegsdrucks (PEEP oder CPAP) der endexspiratorische Kollaps alveolärer Bezirke vermieden und kollabiertes Lungengewebe zum Teil wieder eröffnet werden, was zu einer Verbesserung des Ventilations-Perfusions-Verhältnisses führt. Dadurch wird zusätzlich die Compliance verbessert, was eine Abnahme der Atemarbeit und damit des atemmuskulären Sauerstoffverbrauchs nach sich zieht. Durch zusätzliche inspiratorische Druckunterstützung (z. B. PSV) kann die Atemarbeit weiter reduziert werden.

Es gibt 2 wesentliche Indikationsbereiche, für die die NPPV bei der hypoxämischen ARI fest etabliert ist: das kardiale Lungenödem und die ARI bei Immunsuppression. Bei schweren Störungen kann das kardiale Lungenödem auch mit Hyperkapnie und respiratorischer Azidose einhergehen (•Abb. 15.15). Für beide Erkrankungen konnte eine Reduzierung der Intubationsrate und der Mortalität in Studien nachgewiesen werden. Die Anwendung der NPPV sollte daher unter der Beachtung von Kontraindikationen bei diesen beiden Krankheitsbildern in jedem Fall erwogen werden. Beim kardialen Lungenödem ist auch die Wirksamkeit durch die alleinige Applikation von CPAP wissenschaftlich gut belegt, selbst wenn eine Hyperkapnie vorliegt. Auch bei anderen Erkrankungen (z. B. Pneumonie, Trauma, Atelektase) kann die Anwendung einer NPPV günstige Effekte erzielen. Die Erfolgsrate ist 

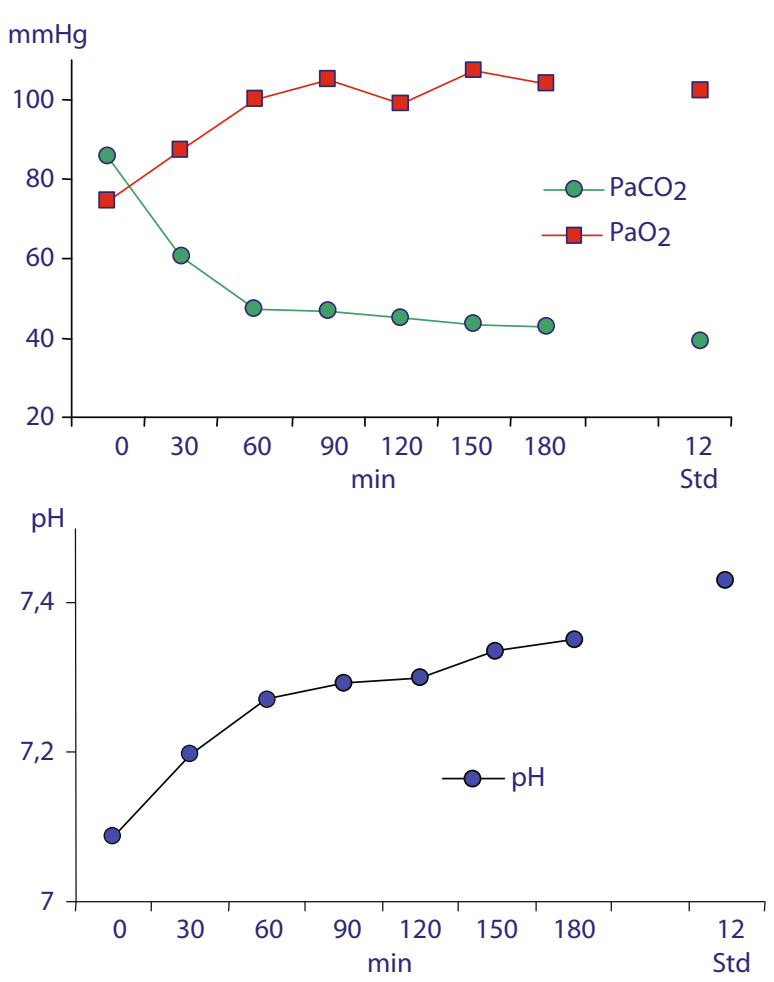

- Abb. 15.15a,b. 63-jährige Patientin mit akutem Lungenödem und schwerer respiratorischer Insuffizienz mit respiratorischer Azidose. Akuteinleitung auf eine nichtinvasive Beatmung (NIV) auf der Intensivstation. Der $\mathrm{P}_{\mathrm{a}} \mathrm{O}_{2}$ ist zu Beginn der NIV (0 min) durch die supplementäre Sauerstoffgabe normalisiert. Nach $3 \mathrm{~h}$ konnte die NIV abgesetzt werden, nach $12 \mathrm{~h}$ war eine Verlegung mit normalisierter Blutgasanalyse auf eine Normalstation möglich

jedoch weniger regelhaft. Das ARDS kann in der Hand eines erfahrenen Zentrums bei ausgewählten Patienten ebenfalls eine Indikation zur Anwendung der NPPV darstellen. In der Regel bedarf dieses Krankheitsbild jedoch der invasiven Beatmung sowie einer komplexen intensivmedizinischen Behandlung.

In Deutschland und anderen Ländern werden zunehmend mehr Patienten mit schwerer chronischer respiratorischer Insuffizienz einseitig oder doppelseitig lungentransplantiert. Nicht selten kommt es bei diesen Patienten während des Wartens auf ein passendes Organ zu einer klinischen Verschlechterung mit Ausbildung einer akuten oder zumindest rasch progredienten respiratorischen Insuffizienz, was die Dringlichkeitsstufe zur Lungentransplantation erhöht. In einer solchen Situation kann die NPPV zur Anwendung kommen und die Zeit bis zur Transplantation überbrücken (»bridging to transplantation«). Typische Krankeitsbilder sind hier neben der Lungenfibrose auch die Mukoviszidose und die COPD, sodass eine NPPV als Bridging sowohl bei der hypoxämischen als auch bei der hyperkapnischen respiratorischen Insuffizienz zum Einsatz kommen kann.

\subsubsection{Nichtinvasive Beatmung bei schwieriger Entwöhnung vom Respirator, während der Postextubationsphase und perioperativ}

Die NPPV eignet sich nicht nur für Patienten mit akuter respiratorischer Insuffizienz, bei denen noch keine Form der invasiven Beatmung eingesetzt wurde; sie kann auch indiziert sein bei Patienten mit schwieriger Entwöhnung vom Respirator, bei erneuter respiratorischer Insuffizienz nach initial erfolgreicher Extubation (Postextubationsversagen) sowie bei postoperativer respiratorischer Insuffizienz.

\section{NPPV bei schwieriger Entwöhnung vom Respirator}

Die Entwöhnung vom Respirator (Weaning) nach erfolgreicher Behandlung einer akuten intubationspflichtigen respiratorischen Insuffizienz gelingt in den meisten Fällen problemlos. Es gibt jedoch in ca. 10\% der Beatmungsfälle Schwierigkeiten, den Patienten vom Respirator zu entwöhnen. Eine »schwierige Entwöhnung « liegt dann vor, wenn der Patient innerhalb von 7 Tagen nicht entwöhnt werden kann. Patienten, die länger als 7 Tage beatmet werden müssen, sind »langzeitbeatmet «. Die Ursachen für eine schwierige Entwöhnung sind vielfältig. Häufig besteht bereits im Vorfeld eine chronische hyperkapnische respiratorische Insuffizienz, die eine Entwöhnung erschwert. Dies trifft insbesondere bei der COPD zu. Andere wesentliche Ursachen sind thorakal restriktive Erkrankungen, Adipositas, begleitende Herzinsuffizienz, die »critical illness«-Neuropathie, nicht erkannte zugrunde liegende neuromuskuläre Erkrankungen wie die amyotrophe Lateralsklerose oder Komplikationen nach Trauma oder Operationen.

Mit zunehmender Dauer der invasiven Beatmung steigt auch die Komplikationsrate, insbesondere die der tubusassoziierten Pneumonie, was die Prognose der Patienten erheblich einschränken kann. Aus diesem Grund stellt der Wechsel von einem invasiven auf einen nichtinvasiven Beatmungszugang eine sinnvolle Rationale dar. Allerdings ist der Einsatz der NPPV beim Weaning von Patienten mit hyperkapnischer respiratorischer Insuffizienz erfolgversprechender als bei Patienten mit hypoxämischer respiratorischer Insuffizienz. Gute wissenschaftliche Daten liegen insbesondere für die COPD vor. Dabei konnte der Wechsel auf eine NPPV die Komplikationsund Tracheotomierate senken, die Erfolgsrate für die Entwöhnung steigern und schließlich auch die Prognose der Patienten verbessern. Voraussetzung für eine Anwendung der NPPV im Weaning ist der kooperative Patient, das Fehlen von Kontraindikationen (s. oben) sowie Erfahrung mit der NPPV im Entwöhnungsprozess.

In diesem Zusammenhang sei erwähnt, dass es für die Entwöhnung vom Respirator spezialisierte »Weaning- 
Zentren« gibt, die Patienten mit Langzeitbeatmung speziell zur Entwöhnung vom Respirator aus anderen Kliniken übernehmen. Dabei gelingt nicht selten die Entwöhnung oder Umstellung auf eine intermittierende häusliche NPPV auch bei Patienten, die über viele Wochen oder sogar Monate invasiv langzeitbeatmet waren.

\section{NPPV während der Postextubationsphase}

Die Inzidenz einer notwendigen Reintubation zur Behandlung einer erneuten ARI nach primär erfolgter Extubation beträgt in Abhängigkeit von dem untersuchten Patientenkollektiv 3-24\%. Ein solches "Postextubationsversagen« geht mit deutlich erhöhter Komplikationsrate und Mortalität einher. Die NPPV kann hier bei Patienten mit entsprechenden Risikofaktoren die Prognose verbessern. Solche Risikofaktoren sind die Hyperkapnie, die Hypersekretion, die COPD, bekannte Schwierigkeiten bei der Entwöhnung vom Respirator, das fortgeschrittene Alter, eine Herzinsuffizienz sowie eine schwere Adipositas. Bei diesen Patienten kann durch den Einsatz der NPPV die Reintubationsrate reduziert und die Mortalität gesenkt werden. Vorausetzung ist allerdings ein frühzeitiger Beginn der NPPV. Erfolgt der Beginn der NPPV zu spät ist eine Reintubation häufig notwendig und darf nicht hinausgezögert werden. Eine weitere Voraussetzung ist die Erfahrung des Teams auf der Intensivstation mit der NPPV.

\section{Perioperative und periinterventionelle Anwendung der NPPV}

Im Rahmen einer Allgemeinanästhesie mit Rückenlage und maschineller Beatmung kann es zu einer Abnahme der funktionellen Residualkapazität mit exspiratorischem Verschluss der kleinen Atemwege kommen. Die Folge ist eine Ausbildung von Atelektasen mit Entwicklung eines intrapulmonalen Rechts-links-Shunts und konsekutiver Verschlechterung der Oxygenierung. Eine vorbestehende chronische respiratorische Insuffizienz sowie eine schmerzbedingte Schonatmung können weitere Bedingungen für eine gestörte respiratorische Funktion darstellen. Durch den postoperativen Einsatz einer NPPV oder zum Teil auch nur durch CPAP lässt sich eine Verbesserung der respiratorischen Situation erzielen. Dies konnte auch für Patienten mit Lungenteilresektion oder Lungentransplantation gezeigt werden. Durch die Verbesserung der respiratorischen Funktion im Rahmen einer CPAPbzw. NPPV-Therapie können Reintubationen vermieden, Liegezeiten in der Klinik reduziert und die Prognose der Patienten verbessert werden.

An dieser Stelle sei auch erwähnt, dass nicht selten $\mathrm{Pa}$ tienten mit vorbestehender Hyperkapnie im Sinne einer chronisch ventilatorischen Insuffizienz operiert werden müssen. In Abhängigkeit von der Dringlichkeit der Ope- ration sollte hier bereits präoperativ eine Einleitung der NPPV erfolgen, um ein Weaning mit möglichst kurzer invasiver Beatmungszeit zu ermöglichen. Schließlich kann die NPPV auch periinterventionell die respiratorische Situation verbessern und entsprechende Interventionen risikoärmer erlauben, z. B. eine Bronchoskopie oder die Anlage einer perkutanen Gastroenterostomie bei neuromuskulären Patienten mit bereits manifester Atempumpinsuffizienz.

\subsubsection{Nichtinvasive Beatmung bei chronischer respiratorischer Insuffizienz}

Der Einsatz einer NPPV zur Therapie der chronischen respiratorischen Insuffizienz (CRI) erfolgt als Langzeittherapie. Dabei wird die Beatmung in der Klinik - in der Regel in einem spezialisierten Zentrum - eingeleitet und zu Hause vom Patienten fortgeführt (häusliche Beatmung oder Heimbeatmung). Weitere stationäre Aufnahmen erfolgen zur Routinekontrolle - z. B. im Abstand von 6 Monaten - sowie bei klinischer Verschlechterung, bei Maskenproblemen, Intoleranz der Beatmung oder Exazerbationen der Grunderkrankung. Im Rahmen einer häuslichen Beatmung wird die NPPV intermittierend eingesetzt, d. h. nach erfolgtem Beatmungsintervall über einige Stunden steht der erholten Atempumpe eine vermehrte Funktionsreserve zur Verfügung. Üblicherweise wird die häusliche Beatmung während des Schlafs durchgeführt. Dies hat den Vorteil, dass die zum Teil schweren schlafbezogenen Atemstörungen ebenfalls suffizient behandelt werden können. Zum Teil kann die Beatmungszeit individuell jedoch auch deutlich verlängert werden, insbesondere bei Patienten mit neuromuskulären Erkrankungen, die sich gelegentlich bis zu $20 \mathrm{~h} / \mathrm{Tag}$ oder sogar noch länger beatmen.

Die Wirkungsweise der intermittierenden NPPV im Rahmen einer häuslichen Beatmung ist noch nicht komplett verstanden. Bekannt ist aber, dass die NPPV die Blutgase nicht nur während der Anwendung der NPPV verbessern kann, sondern dass auch im darauf folgenden Spontanatmungsinterval die Blutgase auf dem verbesserten Niveau stabil gehalten werden oder sich sogar noch weiter verbessern können, was auf eine Zunahme des Tidalvolumens im Spontanatmungsinterval nach erfolgter NPPV zurückzuführen ist. Die NPPV bei CRI ist also nicht nur als supportive Maßnahme zu verstehen, sondern vielmehr als therapeutisches Konzept, das bei intermittierender Anwendung die respiratorische Situation sowohl unter der Beatmung als auch in der nachfolgenden Spontanatmung verbessert.

Es werden 3 Mechanismen für diese Wirkungsweise diskutiert: die Erholung einer erschöpften Atemmuskulatur, die Wiedereröffnung von Mikroatelektasen mit 
Abnahme der Atemarbeit sowie ein sogennantes »Resetting « der zentralen Chemorezeptoren im Atemzentrum, was eine Veränderung des Atemantriebs bewirkt. Das genaue Zusammenspiel dieser diskutierten Faktoren, ihre Regelmechanismen und Stellgrößen sowie der Einfluss der zugrunde liegenden Erkrankung sind jedoch noch nicht verstanden.

Epidemiologische Daten aus Europa zeigen, dass im Jahre $2001 \mathrm{mehr}$ als 21.000 Patienten mit häuslicher Beatmung erfasst wurden. $\mathrm{Zu}$ beachten ist jedoch, dass nur etablierte Beatmungszentren in der Erhebung berücksichtigt wurden und weniger als $70 \%$ der ausgewiesenen Beatmungszentren an der Erhebung teilgenommen haben. Daher liegt die Zahl der Patienten, die eine häusliche Beatmung durchführen, vermutlich deutlich höher, zumal außerdem von einer steigenden Inzidenz ausgegangen werden muss. Die wesentlichen Erkrankungsgruppen, die von einer häuslichen Beatmung profitieren, sind die COPD, thorakal-restriktive Erkrankungen wie die Kyphoskoliose oder das posttuberkulöse Syndrom (PostTbc-Syndrom) und neuromuskuläre Erkrankungen. Aber auch das Adipositas-Hypoventilationssyndrom und seltenere Indikationen wie die Mukoviszidose haben an Bedeutung für die häusliche NPPV gewonnen.

Internationale Richtlinien zur Einleitung einer NPPV bei den verschiedenen Erkrankungen wurden 1999 auf einer Konsensuskonferenz festgelegt. Sie sind in - Tab. 15.4 abgebildet. Hier handelt es sich ausschließlich um Expertenempfehlungen. Evidenzbasierte Empfehlungen für die Anwendung der NPPV bei CRI gibt es nicht. Eine individuelle Entscheidung, ob und wann eine Einleitung einer NPPV erfolgen sollte, ist somit unabdingbar.

\section{Nichtinvasive Beatmung bei neuromuskulären Erkrankungen}

Patienten mit neuromuskulären Erkrankungen waren die ersten, die mittels NPPV auf eine häusliche Beatmung eingestellt wurden. Historisch leitet sich die Tradition bei diesen Patienten von der Beatmung in der "eisernen Lunge« ab, was insbesondere für Patienten mit Poliomyelitis in den 30er- bis 50er-Jahren des 20. Jahrhunderts eine wichtige Therapieoption darstellte.

Es gibt eine Vielzahl von neuromuskulären Störungen, die im Verlauf der Erkrankung eine Insuffizienz der Atemmuskulatur entwickeln. Die wesentlichen sind in der - Tab. 15.5 dargestellt.

Die Vielzahl der neuromuskulären Erkrankungen und der unterschiedliche Zeitpunkt, an dem die Atemmuskulatur betroffen ist, machen es schwierig, einheitliche Richtlinien hinsichtlich des richtigen Zeitpunkts einer NIV-Einleitung zu definieren. Eine Konsensuskonferenz hat sich 1999 auf bestimmte Kriterien geeinigt, bei denen eine NIV eingeleitet werden sollte (•Tab. 15.4). Aufgrund der unterschiedlich schnellen Progredienz muss diese allgemeine Definition der Einleitungskriterien heute mehr und mehr einer individuellen Betrachtungsweise weichen. Daher empfiehlt es sich, Patienten mit neuromuskulären Erkrankungen frühzeitig in einem erfahrenen Zentrum zur Evaluation einer möglichen NPPV vorzustellen, um den individuell optimalen Zeitpunkt zur Einleitung einer NPPV zu finden.

Grundsätzlich muss bei neuromuskulären Erkrankungen berücksichtigt werden, dass neben der Schwäche der Inspirationsmuskulatur auch die Exspirationsmuskulatur in ihrer Kraft erheblich eingeschränkt sein kann. Neben der ventilatorischen Insuffizienz, die grundsätzlich der Beatmung zugänglich ist, kommt es bei diesen Patienten auch zu einem abgeschächten Hustenstoß, zu vermehrter Sekretretention und mitunter zu Aspirationen und Aspirationspneumonien. Aus diesem Grund muss bei neuromuskulären Patienten neben der NPPV auch die Notwendigkeit zur Physiotherapie oder maschinellen Hustenhilfe überprüft werden. Neuromuskuläre Patienten sollten daher immer in einem entsprechend erfahrenen Zentrum behandelt werden.

- Tab. 15.4. Richtlinien zur Einleitung einer nichtinvasiven Beatmung

Thorakal-restriktive und neuromuskuläre Erkrankungen

Symptome einer nächtlichen Hypoventilation (z. B. Tagesmüdigkeit, erhöhte Einschlafneigung, morgendliche Kopfschmerzen) und eines der folgenden physiologischen Kriterien

- $\mathrm{P}_{\mathrm{a}} \mathrm{CO}_{2} \geq 45 \mathrm{mmHg}$

- nächtliche $\mathrm{O}_{2}$-Sättigung $\leq 88 \%$ länger als $5 \mathrm{~min}$

- PImax $\leq 5,88 \mathrm{kPa}\left(60 \mathrm{cmH}_{2} \mathrm{O}\right)$ oder FVC $<50 \%$ bei progressiver neuromuskulärer Erkrankung
Chronisch-obstruktive Lungenerkrankung

1. Symptome einer nächtlichen Hypoventilation (z. B. Tagesmüdigkeit, erhöhte Einschlafneigung, morgendliche Kopfschmerzen) und eines der folgenden physiologischen Kriterien

- $\mathrm{P}_{\mathrm{a}} \mathrm{CO}_{2} \geq 55 \mathrm{mmHg}$

- $\mathrm{P}_{\mathrm{a}} \mathrm{CO}_{2 \text { von }} 50-54 \mathrm{mmHg}$ und nächtliche $\mathrm{O}_{2}$-Entsättigung

$\left(\mathrm{O}_{2}\right.$-Sättigung $\leq 88 \%$ für 5 kontinuierliche Minuten bei einer $\mathrm{O}_{2}$-Therapie $\geq 2 \mathrm{l} / \mathrm{min}$ )

- $\mathrm{P}_{\mathrm{a}} \mathrm{CO}_{2}$ von 50-54 mmHg und mehr als 2 Krankenhausaufenthalte in den letzten 12 Monaten aufgrund einer respiratorischen Exazerbation mit Hyperkapnie 


\section{Amyotrophe Lateralsklerose}

Die amyotrophe Lateralsklerose (ALS) ist die häufigste schwere neuromuskuläre Erkrankung im Erwachsenenalter. Die Schädigung des 1. und 2. Motoneurons führt zum charakteristischen Bild gleichzeitig auftretender schlaffer und spastischer Paresen in unterschiedlichen Körperregionen. Durch den Befall der Atemmuskulatur, die bei 10-20\% der Patienten bereits sehr früh im Krankheitsverlauf auftritt, kommt es fast regelhaft zu einer Atempumpinsuffizienz. Des Weiteren wird die bulbäre Verlaufsform mit Sprach- und Schluckstörungen von der nichtbulbären Verlaufsform unterschieden.

Die Erfolgsrate der NPPV ist bei der nichtbulbären Verlaufsform günstiger, da es bei Lähmung der Schlundmuskulatur unter Anwendung eines inspiratorischen positiven Drucks zu einem Kollaps der oberen Atemwege kommen kann. Dennoch können aber auch diese Patienten in der Hand eines Geübten auf eine häusliche NPPV eingeleitet werden. Durch die NPPV kann bei ALS-Patienten die Prognose verbessert werden. Allerdings weisen Studiendaten darauf hin, dass nur mit einem kleineren Teil der Patienten mit diagnostizierter ALS die Möglichkeiten einer häuslichen NPPV besprochen werden.

\section{Muskeldystrophie Duchenne}

Patienten mit einer Muskeldystrophie Duchenne (DMD) entwickeln bis zum 16. Lebensjahr in mehr als $80 \%$ der Fälle eine CRI. Der Tod tritt normalerweise ohne Beatmung aufgrund einer respiratorischen Insuffizienz zwischen dem 16. und dem 25. Lebensjahr ein. Mittels NIV werden Verläufe bis zum 35. Lebensjahr erzielt. Ein Großteil der nichtinvasiv beatmeten DMD-Patienten erreicht heute das 30. Lebensjahr (•Abb. 15.16). Eine prophylaktische NPPV vor Entstehung einer respiratorischen Insuffizienz kann nach aktueller Studienlage nicht empfohlen werden. Neue wissenschaftliche Daten zeigen darüber

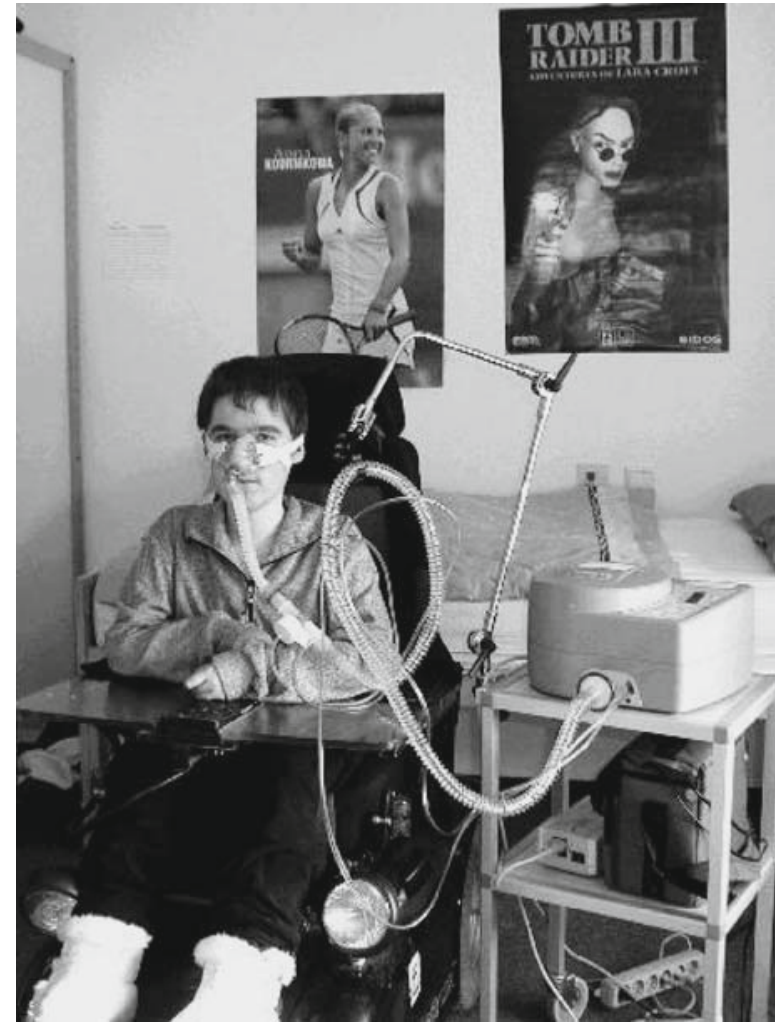

- Abb. 15.16. Häusliche Beatmung bei einem Patienten mit Muskeldystrophie Duchenne

- Tab. 15.5. Neuromuskuläre Erkrankungen mit möglicher chronischer ventilatorischer Insuffizienz

\begin{tabular}{l|l}
\hline Erkrankungsgruppe & Beispiele \\
\hline Muskeldystrophien & Duchenne, Becker-Kiener, Gliedergürtelmuskeldystrophie \\
\hline Myotone Dystrophien & Curschmann-Steinert \\
\hline Metabolische Myopathien & Glykogenose Typ II (M. Pompe), Glykogenose TypV (M. McArdle) \\
\hline Myositiden & Polymyositis, Dermatomyositis, Einschlusskörperchenmyositis \\
\hline Erkrankungen der neuromuskulären Übertragung & Myasthenia gravis pseudoparalytica \\
\hline Polyneuropathien & $\begin{array}{l}\text { Hereditäre motorisch-sensorische Neuropathien, entzündliche } \\
\text { Neuropathien }\end{array}$ \\
\hline Erkrankungen des 1. und 2. Motoneurons & Amyotrophe Lateralsklerose \\
\hline Erkrankungen des 2. Motoneurons & Postpoliosyndrom \\
\hline $\begin{array}{l}\text { Erkrankungen des 2. motorischen Neurons (Vorderhornerkran- } \\
\text { kungen) }\end{array}$ & Spinale Muskelatrophie \\
\hline Phrenikusparese (idiopathisch, traumatisch, entzündlich, tumorös) & Zwerchfelllähmung \\
\hline
\end{tabular}


hinaus, dass ein Atemmuskeltraining sinnvoll sein und den Zeitpunkt hinauszögern kann, an dem eine NPPV notwendig wird. Weitere Studien sind jedoch notwendig, um die Bedeutung eines solchen Atemmuskeltrainings zu beurteilen.

\section{Nichtinvasive Beatmung bei thorakal-restriktiven Erkrankungen}

Thorakal-restriktive Erkrankungen zeichnen sich durch eine Thoraxdeformität mit lungenfunktioneller Restriktion und eingeschränkter Compliance des respiratorischen Systems aus. Durch die Deformität kommt es aber auch zu einer Konfigurationsänderung der Atemmuskulatur und insbesondere des Zwerchfells. Die Folge ist eine ventilatorische Insuffizienz. Die Ursachen für eine Thoraxdeformität sind vielfältig. Am häufigsten sind ein posttuberkulöses Syndrom oder eine Kyphoskoliose Ursache der thorakal-restriktiven Erkrankung.

Das posttuberkulöse Syndrom ist ein Sammelbegriff für die Residuen einer induzierten Defektheilung der Tuberkulose zum Zeitpunkt vor der Entwicklung der antituberkulösen Medikation. Thorakoplastische Eingriffe, extrapleurale Pneumolysen oder eine Phrenikusexhairese waren zur Behandlung der Tuberkulose weit verbreitet und führten in der Folge zu einer Thoraxdeformität mit konsekutiver ventilatorischer Insuffizienz, häufig allerdings erst viele Jahre nach der Therapie. Kyphoskoliosen enstehen idiopathisch im Wachstumsalter, kongenital sowie in der Folge neuromuskulärer Erkrankungen.

Die häusliche NPPV kann insbesondere bei Patienten mit thorakal-restriktiven Erkrankungen und CRI günstige Effekte haben. Neben einer Verbesserung der Blutgase und einer potenziellen Rückbildung einer sekundären pulmonalen Hypertonie kann die NPPV die Schlafqualität und Lebensqualität sowie das Langzeitüberleben verbessern. Die Wirksamkeit der NPPV ist sowohl in der klinischen Praxis als auch in unkontrollierten Studien so eindrücklich, dass sich kontrollierte Studien aus ethischen Gründen verbieten. Alle Patienten mit chronischer Hyperkapnie und Thoraxdeformität sollten daher in jedem Fall hinsichtlich der Möglichkeiten einer häuslichen Beatmung evaluiert werden.

\section{Nichtinvasive Beatmung bei Obesitas- Hypoventilation}

Ein Obesitas-Hypoventilationssyndrom (OHS) ist gekennzeichnet durch eine Adipositas $\left(\mathrm{BMI}>30 \mathrm{~kg} / \mathrm{m}^{2}\right)$ verbunden mit einer Tageshyperkapnie $\left(\mathrm{P}_{\mathrm{a}} \mathrm{CO}_{2} \geq 45 \mathrm{mmHg}\right)$. Dieses Krankheitsbild wird oft als Pickwick-Syndrom bezeichnet, in Anlehnung an die Romanfigur »little fat Joe« von Charles Dickens. Die Ursache des OHS ist noch nicht hinreichend geklärt. Sowohl atemmechanische Veränderungen mit verminderter Compliance, erhöhter Resistance und konsekutiv mit einer vermehrten Atemarbeit als auch Störungen des Atemantriebs werden ursächlich diskutiert. Viele, aber nicht alle Patienten mit OHS leiden gleichzeitig an einem Schlafapnoesyndrom. Entsprechend stehen symptomatisch neben der Dyspnoe und einer eingeschränkten Belastbarkeit Beschwerden einer schlafbezogenen Atemstörung wie erhöhte Tagesmüdigkeit im Vordergrund.

Ebenso wie bei thorakal-restriktiven Erkrankungen kann die NPPV eine deutliche Verbesserung der Blutgasanalyse mit Normalisierung der $\mathrm{P}_{\mathrm{a}} \mathrm{CO}_{2}$-Werte bewirken. Auch wenn kontrollierte Studien fehlen, darf ähnlich wie bei Patienten mit thorakal-restriktiven Erkrankungen von einer Verbesserung des Langzeitüberlebens und der Lebensqualiät ausgegangen werden. Im Vergleich zu anderen Erkrankungen mit chronischem Hypoventilationssyndrom ist therapeutisch allerdings nicht nur ein ausreichend hoher Inspirationsdruck zur Augmentierung der alveolären Ventilation, sondern auch ein ausreichend hoher Exspirationsdruck (z. T. bis zu $10 \mathrm{cmH}_{2} \mathrm{O}$ und mehr) zur Vermeidung eines Kollapses der oberen Atemwege insbesondere bei gleichzeitig bestehendem obstruktivem Schlafapnoesyndrom wichtig.

\section{Nichtinvasive Beatmung bei fortgeschrittener COPD}

Der Einsatz der NPPV bei CRI infolge einer COPD ist sehr umstritten. Nationale und internationale Leitlinien zur Diagnostik und Therapie der COPD empfehlen keinen Einsatz der NPPV zur Therapie der stabilen Hyperkapnie bei COPD-Patienten. Hintergrund ist eine Reihe von Studien, die durch den Einsatz der NPPV nur geringradige und inkonsistente Verbesserungen von Untersuchungsparametern wie Blutgasanalyse, Schlafqualität, körperliche Belastbarkeit oder globale Inspirationskraft erzielen konnten. Verbesserungen der Lungenfunktion ergaben sich ebenfalls nicht, was bei der strukturellen Lungenerkrankung COPD auch nicht zu erwarten war. Noch wichtiger ist allerdings die Erkenntnis, dass die zusätzlich zur Sauerstofflangzeittherapie initiierte NPPV keinen Überlebensvorteil im Vergleich zur alleinigen Anwendung einer Sauerstofflangzeittherapie bietet.

Diese wissenschaftlichen Erkenntnisse müssen jedoch vor dem Hintergrund der eingesetzten Beatmungsstragie sehr kritisch betrachtet werden. In den meisten Studien wurden nämlich nur assistierte Beatmungsverfahren mit relativ geringen Inspirationsdrücken (IPAP $10-18 \mathrm{cmH}_{2} \mathrm{O}$ ) gewählt. Bei Patienten mit normaler Lungenstruktur, wie z.B. bei solchen mit neuromuskulären Erkrankungen, kann mit diesen Inspirationsdrücken 
eine verbesserte alveoläre Ventilation mit konsekutiver Normokapnie erzielt werden, nicht aber bei der COPD. Jüngere Studien haben hingegen deutlich gezeigt, dass mit einer kontrollierten Beatmung und höheren Inspirationsdrücken (im Mittel zwischen 20 und $30 \mathrm{cmH}_{2} \mathrm{O}$ ) eine deutliche Abnahme des $\mathrm{P}_{\mathrm{a}} \mathrm{CO}_{2}$ erzielt werden kann. Interessanterweise führt diese Abnahme des $\mathrm{P}_{\mathrm{a}} \mathrm{CO}_{2}$ unter der intermittierenden NPPV auch zu einer Verbesserung der alveolären Ventilation mit Zunahme des Tidalvolumens und Abnahme des $\mathrm{P}_{\mathrm{a}} \mathrm{CO}_{2}$ auch im nachfolgenden Spontanatmungsintervall, sodass für diese Beatmungstechnik auch Verbesserungen für Outcome-Parameter zu postulieren sind. Deutliche Verbesserungen der Lebensqualität wie bei Patienten mit restriktiven Erkrankungen konnten kürzlich gezeigt werden. Allerdings fehlen noch kontrollierte Studien, die das Langzeitüberleben erfassen. Eine solche multizentrische Studie ist kürzlich in Deutschland gestartet worden. Ihre Ergebnisse werden mit Spannung erwartet.

Die klinische Erfahrung zeigt jedoch, dass bei Anwendung einer NPPV im kontrollierten Modus und mit hohen Beatmungsdrücken mit dem Ziel einer deutlichen Reduktion des $\mathrm{P}_{\mathrm{a}} \mathrm{CO}_{2}$ oder sogar einer Normokapnie wesentliche klinische Besserungen bei Patienten mit stabiler hyperkapnischer COPD zu erzielen sind. Dies betrifft die Verbesserung der subjektiven Schlafqualität, der Symptome des Patienten sowie die Vermeidung einer Aufnahme auf die Intensivstation im Falle einer Exazerbation. Auch wenn kontrollierte Studien noch ausstehen, rechtfertigen diese Beobachtungen wie auch die Daten zahlreicher unkontrollierter Studien einen Therapieversuch mittels NPPV bei diesen Patienten. Eine Vielzahl epidemiologischer Daten aus Europa zeigen entsprechend, dass - wenn auch mit erheblichen nationalen Unterschieden - vergleichbar viele Patienten mit COPD einer NPPV zugeführt werden wie auch Patienten mit neuromuskulären oder thorakal-restriktiven Erkrankungen.

Der Nutzen einer Rehabilitationsmaßnahme mit körperlichem Training bei der fortgeschrittenen COPD ist unumstritten. Eine zunehmende Anzahl von Studien hat diesbezüglich auch den Stellenwert einer NPPV während der körperlichen Belastung im Zuge einer Rehabilitation untersucht. Dabei konnten Dyspnoe, Blutgase und körperliche Leistung unter Belastung verbessert werden. Jüngste Untersuchungen haben zudem dargelegt, dass bei Patienten mit sehr fortgeschrittener COPD und häuslicher NPPV die Awendung der NPPV beim Gehen zu einer Abnahme der Dyspnoe sowie zu einer Verbesserung der Blutgase und der Gehstrecke führt. Dies könnte als Palliativmaßnahme und zur Erhaltung der körperlichen Belastbarkeit bei COPD-Patienten, die auf eine Lungentransplantation warten, sinnvoll sein. Weitere Studien sind jedoch noch notwendig, um den Stellenwert der NPPV bei körperlicher Belatung beurteilen zu können.

\section{Literaturverzeichnis}

American Thoracic Society (2001) International Consensus Conferences in Intensive Care Medicine: noninvasive positive pressure ventilation in acute Respiratory failure. Am J Respir Crit Care Med 163:283-91

Anonymous (1999) Clinical indications for noninvasive positive pressure ventilation in chronic respiratory failure due to restrictive lung disease, COPD, and nocturnal hypoventilation - a consensus conference report. Chest 116:521-34

Antonelli M, Conti G, Rocco M et al. (1998) A comparison of noninvasive positive-pressure ventilation and conventional mechanical ventilation in patients with acute respiratory failure. N Engl J Med 339:429-35

Antonelli M, Conti G, Moro ML et al. (2001) Predictors of failure of noninvasive positive pressure ventilation in patients with acute hypoxemic respiratory failure: a multi-center study. Intensive Care Med 27:1718-28

Antonelli M, Conti G, Esquinas A et al. (2007) A multiple-center survey on the use in clinical practice of noninvasive ventilation as a firstline intervention for acute respiratory distress syndrome. Crit Care Med 35:18-25

Brochard L, Mancebo J, Wysocki M et al. (1995) Noninvasive ventilation for acute exacerbations of chronic obstructive pulmonary disease. N Engl J Med 333:817-22

Brochard L (2003) Mechanical ventilation: invasive versus noninvasive. Eur Respir J Suppl 47:31s-37s

Brochard L, Mancebo J, Elliott MW (2002) Noninvasive ventilation for acute respiratory failure. Eur Respir J 19:712-21

Buyse B, Meersseman W, Demedts M (2003) Treatment of chronic respiratory failure in kyphoscoliosis: oxygen or ventilation? Eur Respir J 22:525-8

Casanova C, Celli BR, Tost L et al. (2000) Long-term controlled trial of nocturnal nasal positive pressure ventilation in patients with severe COPD. Chest 118:1582-90

Clini E, Sturani C, Rossi A et al. (2002) The Italian multicentre study on noninvasive ventilation in chronic obstructive pulmonary disease patients. Eur Respir J 20:529-38

Conti G, Antonelli M, Navalesi P et al. (2002) Noninvasive vs. conventional mechanical ventilation in patients with chronic obstructive pulmonary disease after failure of medical treatment in the ward: a randomized trial. Intensive Care Med 28:1701-7

Diaz O, Begin P, Torrealba B, Jover E, Lisboa C (2002) Effects of noninvasive ventilation on lung hyperinflation in stable hypercapnic COPD. Eur Respir J 20:1490-8

Dreher M, Storre JH, Windisch W (2007) Noninvasive ventilation during walking in patients with severe COPD: a randomised cross-over trial. Eur Respir J 29:930-6

Elliott MW (2004) Non-invasive ventilation for acute respiratory disease. Br Med Bull 72:83-97

Esteban A, Frutos F, Tobin MJ et al. (1995) A comparison of four methods of weaning patients from mechanical ventilation. Spanish Lung Failure Collaborative Group. N Engl J Med 332:345-50

Esteban A, Frutos-Vivar F, Ferguson ND et al. (2004) Noninvasive positive-pressure ventilation for respiratory failure after extubation. $\mathrm{N}$ Engl J Med 350:2452-60

Ferrer M, Bernadich O, Nava S, Torres A. (2002) Noninvasive ventilation after intubation and mechanical ventilation. Eur Respir J 19:959-65

Ferrer M, Esquinas A, Arancibia F et al. (2003) Noninvasive ventilation during persistent weaning failure: a randomized controlled trial. Am J Respir Crit Care Med 168:70-6

Ferrer M, Valencia M, Nicolas JM, Bernadich O, Badia JR, Torres A (2006) Early noninvasive ventilation averts extubation failure in patients at risk: a randomized trial. Am J Respir Crit Care Med 173:164-70

Girault C, Daudenthun I, Chevron V, Tamion F, Leroy J, Bonmarchand G (1999) Noninvasive ventilation as a systematic extubation and 
weaning technique in acute-on-chronic respiratory failure: a prospective, randomized controlled study. Am J Respir Crit Care Med 160:86-92

Hilbert G, Gruson D, Vargas F et al. (2001) Noninvasive ventilation in immunosuppressed patients with pulmonary infiltrates, fever, and acute respiratory failure. $\mathrm{N}$ Engl J Med 344:481-7

Hill NS (2004) Noninvasive ventilation for chronic obstructive pulmonary disease. Respir Care 49:72-87; discussion 87-9

Janssens J, Derivaz S, Breitenstein E et al. (2003) Changing patterns in long-term noninvasive ventilation: a 7-year prospective study in the Geneva Lake area. Chest 123:67-79

Keenan SP, Sinuff T, Cook DJ, Hill NS (2004) Does noninvasive positive pressure ventilation improve outcome in acute hypoxemic respiratory failure? A systematic review. Crit Care Med 32:2516-23

Lightowler JV, Wedzicha JA, Elliott MW, Ram FSF (2003) Non-invasive positive pressure ventilation to treat respiratory failure resulting from exacerbations of chronic obstructive pulmonary disease: Cochrane systematic review and meta-analysis. BMJ 326:185

Lloyd-Owen SJ, Donaldson GC, Ambrosino N et al. (2005) Patterns of home mechanical ventilation use in Europe: results from the Eurovent survey. Eur Respir J 25:1025-31

Mehta S, Hill NS (2001) Noninvasive ventilation. Am J Respir Crit Care Med 163:540-77

Nava S, Ceriana P (2004) Causes of failure of noninvasive mechanical ventilation. Respir Care 49:295-303

Park M, Sangean MC, Volpe MdS et al. (2004) Randomized, prospective trial of oxygen, continuous positive airway pressure, and bilevel positive airway pressure by face mask in acute cardiogenic pulmonary edema. Crit Care Med 32:2407-15

Peter JV, Moran JL, Phillips-Hughes J, Graham P, Bersten AD (2006) Effect of non-invasive positive pressure ventilation (NIPPV) on mortality in patients with acute cardiogenic pulmonary oedema: a meta-analysis. Lancet 367:1155-63

Plant PK, Owen JL, Elliott MW (2000) Early use of non-invasive ventilation for acute exacerbations of chronic obstructive pulmonary disease on general respiratory wards: a multicentre randomised controlled trial. Lancet 355:1931-5

Schönhofer B, Kohler D (2000) Effect of non-invasive mechanical ventilation on sleep and nocturnal ventilation in patients with chronic respiratory failure. Thorax 55:308-13

Schönhofer B, Sortor-Leger S (2002) Equipment needs for noninvasive mechanical ventilation. Eur Respir J 20:1029-36

Schönhofer B (2006). Nicht-invasive Beatmung - Grundlagen und moderene Praxis, 1. Aufl. UNI-MED, Bremen

Simonds AK, Elliott MW (1995) Outcome of domiciliary nasal intermittent positive pressure ventilation in restrictive and obstructive disorders. Thorax 50:604-9

Simonds AK (2003) Home ventilation. Eur Respir J Suppl 47:38s-46s

Storre JH, Seuthe B, Fiechter R et al. (2006) Average volume-assured pressure support in obesity hypoventilation: A randomized crossover trial. Chest 130:815-21

Wijkstra PJ, Lacasse Y, Guyatt GH et al. (2003) A meta-analysis of nocturnal noninvasive positive pressure ventilation in patients with stable COPD. Chest 124:337-43

Winck JC, Azevedo LF, Costa-Pereira A, Antonelli M, Wyatt JC (2006) Efficacy and safety of non-invasive ventilation in the treatment of acute cardiogenic pulmonary edema - a systematic review and meta-analysis. Crit Care 10:R69

Windisch W, Kostic S, Dreher M, Virchow JCJ, Sorichter S (2005) Outcome of patients with stable COPD receiving controlled noninvasive positive pressure ventilation aimed at a maximal reduction of $\mathrm{Pa}\left(\mathrm{CO}_{2}\right)$. Chest 128:657-62

Windisch W, Dreher M, Storre JH, Sorichter S (2006) Nocturnal noninvasive positive pressure ventilation: Physiological effects on spontaneous breathing. Respir Physiol Neurobiol 150:251-60

\subsection{Maschinelle Beatmung}

S. Ellis, M. Höper, T. Welte

\subsubsection{Einleitung}

In der modernen Intensivmedizin hat die maschinelle Beatmung einen wichtigen Stellenwert. Ein großer Teil der Patienten auf operativen und internistischen Intensivstationen wird maschinell beatmet. Im Laufe der Jahre haben sich aufgrund neuerer Erkenntnisse moderne Beatmungskonzepte etabliert, die dem auf der Intensivstation tätigen Personal vertraut sein sollten. Dazu gehört unter anderem die sogenannte "protektive Beatmung " bei ARDS-Patienten, für die im Gegensatz zu anderen Beatmungskonzepten ein Überlebensvorteil gezeigt werden konnte.

Die Vielzahl von Abkürzungen für die unterschiedlichen Beatmungsmodi erschwert gerade für den Anfänger den Zugang zur Respiratortherapie. Es existieren je nach Hersteller unterschiedliche Abkürzungen für den gleichen Beatmungsmodus. Da auf einer Intensivstation aber in der Regel Respiratoren des gleichen Herstellers eingesetzt werden, sind die Bezeichnungen für die Beatmungsmodi letztlich doch überschaubar und werden nach kurzer Zeit zur Routine.

Die folgenden Kapitel geben zunächst einen Überblick über die Indikation zur maschinellen Beatmung und vermittelt Kenntnisse über die praktische Durchführung sowie über Beatmungskomplikationen. Darüber hinaus werden die Beatmung von speziellen Patientengruppen und die Respiratorentwöhnung behandelt. Zum Schluss werden supportive Konzepte erläutert.

\subsubsection{Indikationen zur maschinellen Beatmung}

\section{Oxygenierungsversagen}

Das Oxygenierungsversagen ist auf internistischen Stationen die häufigste Indikation zur maschinellen Beatmung. Patienten mit Pneumonie und akutem Lungenversagen (ALI/ARDS) entwickeln in den betroffenen Lungenarealen ein niedriges Ventilations-Perfusions-Verhältnis (V/Q) und pulmonale Shunts. Aufgrund gestörter pulmonaler hypoxischer Vasokonstriktion durch proinflammatorische Zytokine kann es je nach Ausmaß der Schädigung zu einer schweren Hypoxämie kommen. Patienten mit niedrigem V/Q weisen gut perfundierte Lungenanteile auf, die aber schlecht belüftet sind. Patienten mit Pneumonie weisen in konsolidierten Bereichen nicht belüftete, aber (bedingt durch proinflammatorische Zytokine) gut perfundierte Areale auf, die einen funktionellen Rechts-links-Shunt zur Folge haben. Überwiegt die Shuntkomponente lässt 
sich auch durch eine hohe Sauerstoffkonzentration die Oxygenierung kaum verbessern (• Abb. 15.17). Zur Berechnung des pulmonalen Shunts kann folgende Formel herangezogen werden:

Shuntfraktion QS/QT $=\left(\mathrm{C}_{\mathrm{c}} \mathrm{O}_{2}-\mathrm{C}_{\mathrm{a}} \mathrm{O}_{2}\right) /\left(\mathrm{C}_{\mathrm{c}} \mathrm{O}_{2}-\mathrm{C}_{\mathrm{v}} \mathrm{O}_{2}\right)$

QS Shuntvolumen, QT Herzzeitvolumen, $\mathrm{C}_{c} \mathrm{O}_{2}$ kapillärer Sauerstoffgehalt, $\mathrm{C}_{a} \mathrm{O}_{2}$ arterieller Sauerstoffgehalt, $\mathrm{C}_{v} \mathrm{O}_{2}$ gemischtvenöser Sauerstoffgehalt

Spontanatmende Patienten mit Oxygenierungsversagen erhalten eine Sauerstoffinsufflation über Nasensonde oder Maske. Dabei lässt sich eine inspiratorische Sauerstoffkonzentration $\left(\mathrm{FiO}_{2}\right)$ von bis $\mathrm{zu} 0,5$ erzielen; bei der Verwendung von Masken mit Reservoirbeutel eine $\mathrm{FiO}_{2}$ von bis zu 0,7. In der arteriellen Blutgasanalyse ist ein erniedrigter Sauerstoffpartialdruck bei oft erniedrigtem $\mathrm{PCO}_{2}$ erkennbar, was als Bedarfshyperventilation bei Hypoxämie anzusehen ist. Bei hoher Shuntfraktion oder zunehmend niedrigem V/Q lässt sich die erforderliche Sauerstoffkonzentration nicht mehr über Nasensonden bzw. Gesichtsmasken erzielen, zumal die häufig stark erhöhte Atemarbeit (Tachypnoe und häufig begleitende verminderte Compliance der Lunge) den Sauerstoffbedarf weiter steigert, sodass die betroffenen Patienten von einer Erschöpfung der Atemmuskulatur bedroht sind.

Eine maschinelle Beatmung bietet die Möglichkeit, einerseits eine $\mathrm{FiO}_{2}$ von 1,0 zu applizieren und andererseits über einen positiven endexspiratorischen Druck die funktionelle Residualkapazität zu erhöhen, was bei niedrigem V/Q zu einer Verbesserung der Oxygenierung führt. Ein weiterer Effekt ist die Entlastung der Atempumpe. Bei Patienten mit Lungenödem kann ein erhöhter PEEP den auf die Alveole wirkenden hydrostatischen Kräften entgegenwirken und somit den Gasaustausch deutlich verbessern. Ein wichtiger Unterschied zwischen den hypoxämieauslösenden Mechanismen ist die Tatsache, dass die Sauerstoffgabe bei hohen Shuntfraktionen oft zu einer nur geringen Verbesserung der Oxygenierung beiträgt, während bei
Patienten mit Diffusionsstörungen eine Sauerstoffgabe häufig einen guten Effekt zeigt.

Es existiert bezüglich der Oxygenierung kein Grenzwert, unter dem eine maschinelle Beatmung absolut indiziert ist. Die Entscheidung zur maschinellen Beatmung sollte immer unter Abwägung weiterer klinischer Parameter, wie z. B. der Atemfrequenz, der Beobachtung der Zuhilfenahme der Atemhilfsmuskulatur oder der Gesamteindruck des Patienten, erfolgen und nicht allein aufgrund von einzelnen Parametern wie dem Oxygenierungsindex $\left(\mathrm{P}_{\mathrm{a}} \mathrm{O}_{2} / \mathrm{FiO}_{2}\right)$. Intubationskriterien unter Berücksichtigung des individuellen Gesamteindrucks eines Patienten sind unter anderem:

- Sauerstoffsättigung $<85 \%$ trotz Sauerstoffinsufflation,

- ventilatorisches Versagen unter Therapie (zunehmende Hyperkapnie),

- klinische Zeichen der Atemmuskelpumpenerschöpfung,

- (zunehmende) Atemfrequenz $>30 /$ min unter Therapie,

- neurologische Auffälligkeiten (z. B. motorische Unruhe),

- maligne kardiale Arrhythmien,

- Bewusstseinstörung.

In der Übersicht sind Erkrankungen, die zu einem Oxygenierungsversagen führen können, zusammengestellt.

Erkrankungen, die zu einem Oxygenierungsversagen führen können (Auswahl)

- ARDS

- Pneumonie

- Atelektase

- Pleuraerguss

- (Spannungs-)Pneumothorax

- Lungenödem (toxisch/kardial)

- Lungenkontusion
- Abb. 15.17. Pulmonaler Rechtslinks-Shunt. $P_{\bar{v}} \mathrm{O}_{2}$ gemischtvenöser Sauerstoffpartialdruck, $\mathrm{C}_{\bar{v}} \mathrm{O}_{2}$ gemischtvenöser Sauerstoffgehalt, $\mathrm{C}_{a} \mathrm{O}_{2}$ arterieller Sauerstoffgehalt $\mathrm{P}_{a} \mathrm{O}_{2}$ arterieller Sauerstoffpartialdruck

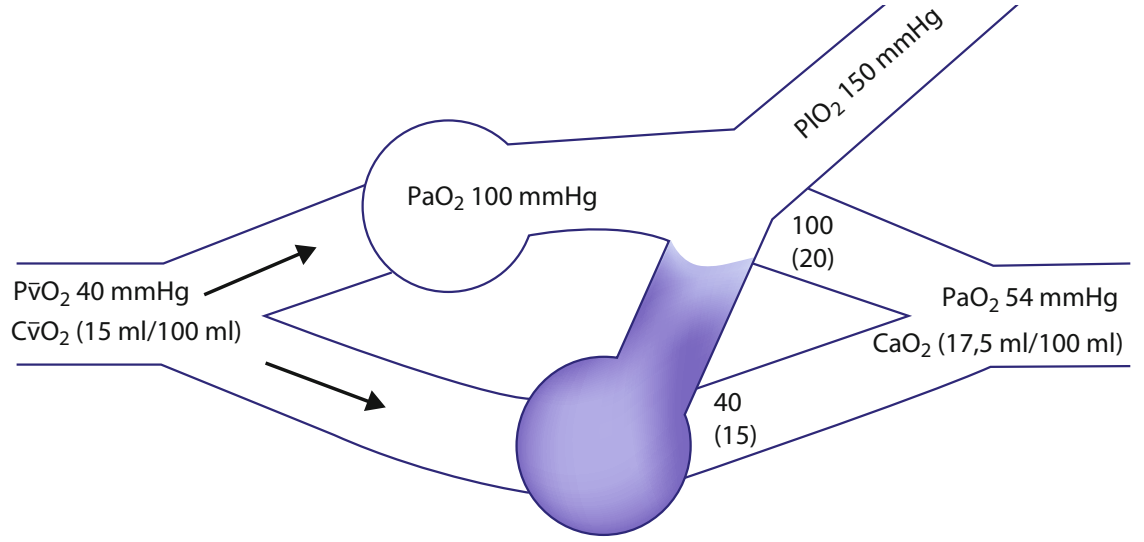




\section{Ventilatorisches Versagen}

Kennzeichnend für ein ventilatorisches Versagen ist eine neu aufgetretene oder aggravierte Hyperkapnie, die mit einer respiratorischen Azidose einhergeht. Typische Erkrankungen, die zu einem ventilatorischen Versagen führen können, sind z. B. die chronisch-obstruktive Lungenerkrankung (meistens im Rahmen einer Exazerbation) oder der Status asthmaticus. Es liegt dabei eine muskuläre Erschöpfung zugrunde, die durch veränderte Atemmechanik und erhöhte Atemarbeit bedingt ist. Dabei sinkt das effektive Atemminutenvolumen und die alveoläre Ventilation nimmt ab. Da zwischen der alveolären Ventilation und dem $\mathrm{P}_{\mathrm{a}} \mathrm{CO}_{2}$ eine inverse Korrelation besteht, kommt es bei muskulärer Erschöpfung zum $\mathrm{P}_{\mathrm{a}} \mathrm{CO}_{2}$-Anstieg.

$\mathrm{Zu}$ einem ventilatorischen Versagen können weiterhin Erkrankungen führen, die mit Atemantriebsstörungen (Apoplex, schwere Elektrolytentgleisungen, schwere Dehydratation, Intoxikation) oder Schwäche der Atempumpe einhergehen, wie z. B. amyotrophe Lateralsklerose oder Myasthenia gravis. Auch Patienten mit schwerer Kyphoskoliose können eine Insuffizienz ihrer Atemmuskulatur entwickeln und sich mit einem ventilatorischen Versagen in der Klinik vorstellen.

Patienten mit einem ventilatorischen Versagen haben nicht zwangsläufig auch eine schwere Hypoxämie, sodass häufig nur ein geringer zusätzlicher Sauerstoffbedarf besteht. Chronisch hyperkapnische Patienten können unter Spontanatmung und Sauerstoffinsufflation aufgrund des nun fehlenden Atemantriebs bei Normoxämie bis hin zum $\mathrm{CO}_{2}$-Koma hypoventilieren. Unter kontrollierter maschineller Beatmung besteht dieses Problem naturgemäß nicht.

Die primäre Therapie des hyperkapnischen Lungenversagens ist die Wiederherstellung einer adäquaten Ventilation. Diese kann invasiv oder nicht invasiv (Masken, Beatmungshelm) appliziert werden. Grundsätzlich ist eine nichtinvasive Beatmung zu bevorzugen, wenn keine Kontraindikationen vorliegen (komatöser Patient, fehlende Husten- und Schluckreflexe, Hypersekretion, Kreislaufinstabilität, maligne kardiale Rhythmusstörungen). Unter einer nichtinvasiven Beatmung sind engmaschige arterielle Blutgasanalysen erforderlich, bei fehlendem Ansprechen (Besserung der Hyperkapnie) oder Hinweisen für ein zusätzliches extrapulmonales Organversagen sollte eine invasive Beatmung erfolgen.

- Tab. 15.6 zeigt eine Auswahl von Erkrankungen die zu einem ventilatorischen Versagen führen können.

\section{Vigilanzstörungen}

Patienten mit Vigilanzstörungen (z. B. Apoplex, hyperosmolares Koma, hepatische Enzephalopathie, schwere Elektrolytengleisungen, Intoxikation) haben je nach Schweregrad mangelnde oder fehlende Schutzreflexe und müssen unter Umständen unverzüglich intubiert werden, um eine drohende Verlegung der Atemwege oder eine Aspiration zu verhindern. Oft liegt bei diesen Patienten auch eine Atemantriebsstörung vor, sodass auch eine Hyperkapnie als Zeichen des ventilatorischen Versagens nachweisbar sein kann. Neben der Sicherung des Atemwegs ist daher auch eine maschinelle Beatmung erforderlich. Diese ist in der Regel unproblematisch, da es sich meist um lungengesunde Patienten handelt.

Die bei komatösen Patienten häufig auftretenden Aspirationen sind meist nicht schwergradig, bakterielle Aspirationspneumonien treten in weniger als $10 \%$ auf, eine routinemäßige Antibiotikatherapie ohne einen sicheren Pneumonienachweis ist nicht notwendig. Eine (säurebedingte) Aspirationspneumonitis manifestiert sich in der Regel erst mit einer Verzögerung von 12-24 (48) h.

\subsubsection{Grundlagen der Beatmung}

Durch die maschinelle Beatmung werden die Druckverhältnisse in der Lunge im Vergleich zur nicht unterstützten Spontanatmung umgekehrt, d.h. bei der Inspiration steigt je nach Respiratoreinstellung der Atemwegsdruck an. Die Exspiration erfolgt wie bei der Spontanatmung passiv. Die alveoläre Ventilation, die durch die Beatmung erreicht wird, hängt neben anatomischen und erkrankungsbedingten Faktoren von den am Respirator eingestellten Parametern ab.

- Tab. 15.6. Erkrankungen, die zu einem ventilatorischen Versagen führen können (Auswahl)

Primäres ventilatorisches Versagen

- Infektexazerbation einer COPD

- Status asthmaticus

- Hypoventilationssyndrome

- Endstadium jeglicher Lungenerkrankung

- Postextubationsversagen durch Versagen der Atemmuskelpumpe

- Neuromuskuläre Erkrankungen

- Chronische Abstoßung nach Lungentransplantation

- Schwere Kyphoskoliose
Sekundäres ventilatorisches Versagen

- Lungenembolie (meist Hyperventilation)

- Kardiogenes und nicht kardiogenes Lungenödem

- Metabolische Azidose (zunächst Hyperventilation, Erschöpfung möglich)

- Schock jedweder Genese (zunächst Hyperventilation, Erschöpfung möglich)

- Postextubationsversagen durch Dystelektaen oder Atelektasen 


\section{Beatmung und Kreislauf}

Die maschinelle Beatmung hat unmittelbaren Einfluss auf das Herz-Kreislauf-System. Die Wirkung ist auf den erhöhten intrathorakalen Druck zurückzuführen, der den venösen Rückfluss vermindert und die rechtsventrikuläre Nachlast erhöht. Auf den linken Ventrikel hat der intrathorakale Druck umgekehrte Wirkung: Die Vorlast wird erhöht und die Nachlast vermindert.

Bei unbeeinträchtigter linksventrikulärer Funktion sinkt das Herzminutenvolumen, es kann aufgrund des relativen »Volumenmangels« $\mathrm{zu}$ einer arteriellen Hypotension kommen, die durch Volumengabe kompensiert werden kann. Bei eingeschränkter linksventrikulärer Funktion kann aufgrund der kombinierten Vor- und Nachlastsenkung ein Anstieg des Herzminutenvolumens zu verzeichnen sein, Positivdruckbeatmung wirkt daher als »therapeutisches « Prinzip bei Linksherzinsuffizienz.

$\mathrm{Zu}$ beobachten sind die Einflüsse der maschinellen Beatmung auf das Herz-Kreislauf-System bei Hypovolämie und intraarterieller Blutdruckmessung: Es entsteht die sogenannte »Volumenmangelkurve«, die durch vorlastbedingte Schwankungen des Schlagvolumens des rechten Ventrikels bedingt ist.

\section{PEEP}

Das exspiratorische Druckniveau ("positive end-expiratory pressure «, PEEP) ist ein Parameter, der bei jeder Beatmungseinstellung einzustellen ist. Der PEEP setzt sich aus 2 Komponenten zusammen: dem extrinsischen PEEP, d. h. dem positiven Atemwegsdruck, der endexspiratorisch durch den Respirator aufrechterhalten wird, und dem intrinsischen PEEP, der in bestimmten Lungenanteilen auftreten kann, wenn durch Beatmung eine dynamische Lungenüberblähung induziert wird, meist als Folge hoher Atemfrequenzen oder durch ungünstiges Verhältnis von Inspiration zu Exspiration (I/E-Verhältnis).

Die Bedeutung des PEEP liegt darin, einen Kollaps der Alveolen zu verhindern und damit die funktionelle Residualkapazität der Lunge zu erhalten. Durch Erhöhung des PEEP kann die funktionelle Residualkapazität und damit die für den Gasaustausch zur Verfügung stehende Oberfläche erhöht werden. Die häufig damit in Zusammenhang stehende Verbesserung der Oxygenierung führt zu der Frage, welcher PEEP-Level für den individuellen Patienten in diesem Moment der günstigste ist.

Es kann für das optimale PEEP-Niveau keine allgemeingültige Empfehlung gegeben werden, da es von vielen Faktoren beeinflusst wird, z. B. durch die Art der Erkrankung, die Compliance der Lunge, den Volumenstatus des Patienten, Erkrankungen anderer Organe (z. B. Hirnödem), den intraabdominellen Druck etc. Ein PEEPNiveau, das primär als optimal angesehen wurde, kann z. B. bei einem ARDS-Patienten im Verlauf der Genesung zu hoch sein. Die fixe Zuordnung von $\mathrm{FiO}_{2}$ und PEEP erscheint ebenfalls nicht sinnvoll, da für jeden Patienten individuelle Bedingungen vorliegen (s. oben). Auch die Frage, ob das PEEP-Niveau eher hoch oder niedrig sein soll, ist nicht abschließend geklärt. Es finden sich jedoch Hinweise dafür, dass ARDS-Patienten, die mit einem hohen PEEP-Niveau ( $>12 \mathrm{cmH}_{2} \mathrm{O}$ ) beatmet werden, in Bezug auf beatmungsfreie Tage oder die Gesamtmortalität keinen Vorteil erlangen.

Die maschinelle Beatmung mit PEEP kann neben den oben erwähnten negativen Auswirkungen auf das Kreislaufsystem auch günstige Effekte auf die kardiale Funktion haben: Bei eingeschränkter systolischer Pumpfunktion des linken Ventrikels kann ein erhöhter intrathorakaler Druck durch Beatmung (insbesondere durch PEEP) funktionell nachlastsenkend wirken, sodass im kardiogenen Schock beispielsweise eine vorsichtige Erhöhung des PEEP versucht werden sollte.

\section{Komplikationen}

Während einer maschinellen Beatmung können verschiedene Komplikationen auftreten, manche akut und lebensbedrohlich, andere unbemerkt und schleichend.

\section{Einfluss auf das Herz-Kreislauf-System}

Der positive Druck, der während der maschinellen Beatmung zyklisch schwankt, hat Auswirkungen auf das Herz-Kreislauf-System (s. oben). Hohe Beatmungsdrücke vermindern den venösen Rückfluss zum Herzen und veringern damit die rechtsventrikuläre Vorlast und somit das Herzzeitvolumen. Dies macht sich vor allem bei volumendepletierten Patienten bemerkbar. Bei Patienten mit einem schweren Cor pulmonale oder akuter Rechtsherzbelastung können aufgrund der erhöhten rechtsventrikulären Nachlast schon moderate Beatmungsdrücke deutlichen Einfluss auf das Herzzeitvolumen haben. Auf hohe PEEP-Werte oder hohe Druckspitzen sollte - wenn möglich - verzichtet werden.

\section{Erhöhter Hirndruck}

Aufgrund des verminderten venösen Rückflusses zum Herzen kann es unter PEEP-Beatmung zum Anstieg des Drucks in den Vv. jugulares internae kommen. Bei Patienten mit erhöhtem Hirndruck kann dies zu einer weiteren Steigerung des Hirndrucks führen. Hohe PEEP-Werte sind bei diesen Patienten zu vermeiden. Die größte Bedeutung hat in diesem Zusammenhang allerdings eine Hyperkapnie: Die früher übliche Hyperventilation zur Hirndrucktherapie wurde zwar mittlerweile weitgehend verlassen, aber eine (akute) Hyperkapnie kann zu dramatischen Hirndruckanstiegen führen und sollte bei Patienten mit intrakranieller Druckerhöhung unbedingt vermieden werden. Gegebenenfalls ist eine Überwachung mittels Hirndrucksonde indiziert. 


\section{Auto-PEEP}

Patienten mit obstruktiven Ventilationsstörungen benötigen im Verhältnis zum Lungengesunden eine längere Exspirationszeit. Erkennbar ist dies an der Flusskurve des Beatmungsgeräts: Der Fluss erreicht den Nullwert nicht, sondern wird durch den nächsten Beatmungszyklus unterbrochen (• Abb. 15.18). Bei hoher Beatmungsfrequenz kann die Exspirationszeit relativ zu kurz sein, sodass das Phänomen der dynamischen Überblähung auftritt. Die funktionelle Residualkapaziät nimmt dabei zu und kann ungünstig hohe Werte erreichen. Es entsteht ein positiver endexspiratorischer Druck in der Lunge (Auto-PEEP oder intrinsischer PEEP), der unabhängig vom externen, respiratorbedingten PEEP auftritt. Die Folgen eines unerkannten intrinsischen PEEP sind arterielle Hypotension, Tachykardie oder eine erschwerte Triggerung des Beatmungsgeräts durch den spontanatmenden Patienten.

Nach Erkennen eines erhöhten intrinsischen PEEP sollte die Exspirationszeit so lange verlängert werden, bis in der Flusskurve des Respirators das Erreichen des Nullwerts erkennbar ist. Dies kann vor allem über eine Verminderung der Atemfrequenz oder auch über eine Verlängerung des I/E-Verhältnisses geschehen. Oft sind entsprechende Patienten chronisch hyperkapnisch, sodass eine damit einhergehende Zunahme der Hyperkapnie toleriert werden kann, solange keine schwergradige Azidose eintritt.

Im Rahmen der Exazerbationsbehandlung bei COPD sollten $\beta$-Mimetika, Anticholinergika, ggf. auch Theophyllin (Cave: kardiale Nebenwirkungen) sowie Steroide verabreicht werden, um eventuell reversible Bronchialobstruktionen zu behandeln. Im Status asthmaticus ist die Gabe von Magnesiumsulfat sinnvoll. Bei putridem Sputum sollte eine antibiotische Therapie mit einem pseudomonaswirksamen Chinolon erfolgen. Bei spontanatmenden Patienten ist ein weiterer Ansatz die schrittweise Erhöhung des externen PEEP bis auf Auto-PEEP-Werte, um die zur Triggerung erforderliche Atemarbeit zu verringern.

In der Übersicht sind die Prävention und Therapie von Auto-PEEP zusammengestellt.

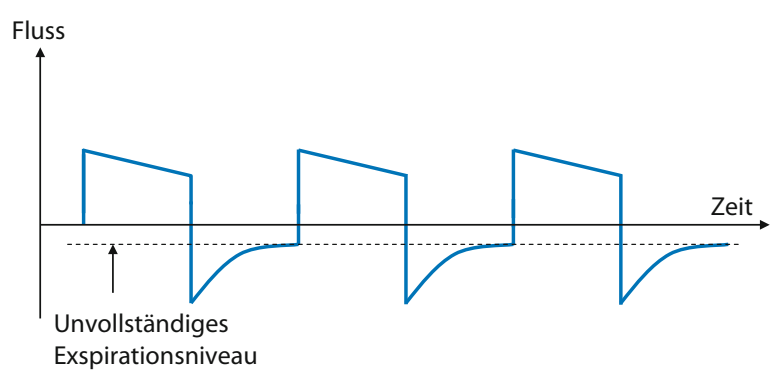

- Abb. 15.18. Fluss-Zeit-Diagramm unter positiver Druckbeatmung bei einem Patienten mit schwerer COPD. Der exspiratorische Fluss fällt vor Beginn der nächsten Inspiration nicht auf Null ab. Dies ist Ausdruck einer unvollständigen Exspiration mit zunehmender Überblähung
Prävention und Therapie von Auto-PEEP

- Sicherstellung einer ausreichend langen Exspirationszeit (Flusskurve)

- I/E-Verhältnis verlängern

- Atemfrequenz verringern

- Therapie bronchialer Obstruktion

- inhalative $\beta$-Mimetika und Anticholinergika, ggfs. Theophyllin

- systemische Steroide (bei Asthma oder COPDExazerbation)

- Sekretabsaugung (u. U. auch bronchoskopisch)

- im Status asthmaticus Magnesiumsulfat

- Verringerung der Atemarbeit des Patienten durch Erhöhung des extrinsischen PEEP auf das gemessene Auto-PEEP-Niveau

\section{Barotrauma}

Der mit der maschinellen Beatmung verbundene positive Beatmungsdruck verursacht in der Lunge ein Barotrauma. Darunter werden (Spannungs-)Pneumothorax, (Spannungs-)Pneumomediastinum und das Hautemphysem zuammengefasst. Diese Komplikationen entstehen meist bei hohen Beatmungsspitzendrücken und sind in der Regel klinisch und radiologisch gut erkennbar. Eine Notfallsituation kann bei einem Spannungspneumothorax unter maschineller Beatmung entstehen. Bei plötzlich einsetzender arterieller Hypotension, Tachykardie, Sättigungsabfall, oberer Einflussstauung und abnehmenden Atemzugvolumina muss an einen Spannungspneumothorax gedacht werden. Häufig bleibt in solchen Situationen keine Zeit, eine Röntgenuntersuchung des Thorax abzuwarten. Bei dringendem Verdacht und typischen klinischen Zeichen sollte die betroffene Thoraxseite unverzüglich entlastet werden. Die präventiven Maßnahmen zur Vermeidung eines Barotaumas sind:

- prompte Korrektur bzw. Vermeidung einer einseitigen Intubation,

- Verwendung niedriger Tidalvolumina (4-6 ml/kgKG),

- Vermeidung von intrinsischem PEEP,

- Vermeidung von Druckspitzen bei Ventilation mit Ambu-Beutel,

- Einstellung einer adäquaten Druckobergrenze am Respirator.

\section{Ventilatorinduzierter Lungenschaden (VILI)}

Bedingt durch hohe Atemzugvolumina kommt es während der Beatmung zum zyklischen Öffnen und Schließen von Alveolen, die auf PEEP-Niveau nicht rekrutiert, also kollabiert sind. Gleichzeitig kommt es zu einer repetitiven Mikrotraumatisierung des Lungengewebes. Damit verbunden ist eine gesteigerte Produktion von inflammatorischen Zytokinen, die ihrerseits $\mathrm{zu}$ einer weiteren 
Schädigung des Lungenparenchyms beiträgt, andererseits aber auch eine systemische inflammatorische Antwort auslösen bzw. unterhalten kann.

Zur Vermeidung eines ventilatorinduzierten Lungenschadens sollten die applizierten Tidalvolumina möglichst klein sein. Um eine ausreichende alveoläre Ventilation sicherzustellen, muss kompensatorisch die Atemfrequenz erhöht werden. Für das ARDS wurden Tidalvolumina mit Werten um $6 \mathrm{ml} / \mathrm{kg}$ (ideales) Körpergewicht als günstig ermittelt, wobei es hinsichtlich des ventilatorassoziierten Lungenschadens möglicherweise keinen unteren Grenzwert gibt.

\section{Sauerstofftoxizität}

Durch die maschinelle Beatmung ist es möglich, die inspiratorische Sauerstoffkonzentration der Atemluft $\left(\mathrm{FiO}_{2}\right)$ auf bis $\mathrm{zu} 100 \% \mathrm{zu}$ erhöhen. Vor allem beim Oxygenierungsversagen ist dies gelegentlich erforderlich, um eine ausreichende Sauerstoffsättigung aufrechtzuerhalten. Aus Tierexperimenten ist bekannt, dass die Ventilation mit reinem Sauerstoff in wenigen Tagen schwere Schäden auch in gesunden Lungen verursachen kann. Es existiert jedoch kein gesicherter $\mathrm{FiO}_{2}$-Schwellenwert, unter dem gefahrlos beatmet werden kann. Im klinischen Alltag wird ein $\mathrm{FiO}_{2}$ von 0,4 oder kleiner als unproblematisch angesehen.

\section{Ventilatorassoziierte Pneumonie}

Patienten, die invasiv beatmet werden, haben ein hohes Risiko, eine ventilatorassoziierte Pneumonie zu erleiden. Diese stellt eine Sonderform der nosokomialen Pneumonie mit deutlich erhöhter Morbidität und Letalität dar. Bezüglich der prophylaktischen Maßnahmen, die bei jedem invasiv beatmeten Patienten getroffen werden sollten, sei auf die Leitlinien des Robert-Koch-Instituts verwiesen (www.rki.de). In der Übersicht sind alle wesentlichen Komplikationen der maschinellen Beatmung zusammengefasst.

\section{Komplikationen der maschinellen Beatmung}

- Arterielle Hypotonie

- Barotrauma

- (Spannungs-)Pneumothorax

- (Spannungs-)Pneumomediastinum

- Hautemphysem

- ventilatorassoziierte Pneumonie (VAP)

- ventilatorassoziierter Lungenschaden (VILI)

- Sauerstofftoxizität

- intubations- bzw. tubusassoziierte Schäden

- Tracheallazeration/-ruptur

- Trachealstenose

- Glottisödem

\section{Monitoring der Beatmung}

Jeder maschinell beatmete Patient sollte aufgrund des geringen Aufwands und der einfachen Verfügbarkeit eine kontinuierliche plethysmographische Überwachung der Sauerstoffsättigung erhalten. Verschlechterungen der Oxygenierung können dabei unverzüglich erkannt werden. Nicht zuverlässig ist diese Methode bei zentralisierten Patienten im Kreislaufschock. Daher ist die Analyse des Sauerstoffsättigungssignals von Bedeutung, da eine unzureichende Signalqualität häufig mit nicht validen Sauerstoffsättigungswerten einhergeht.

Maßgeblicher Indikator der alveolären Ventilation ist der $\mathrm{P}_{\mathrm{a}} \mathrm{CO}_{2}$, der über arterielle Blutgasanalysen bestimmt wird. Diese sollten in der Regel im 4- bis 6-stündigen Intervall durchgeführt werden.

Beatmungsbezogene Informationen, wie beispielsweise der Beatmungsdruck, das Atemminutenvolumen oder das Atemzugvolumen, sind bei jedem Respirator kontinuierlich ablesbar. Die Alarmgrenzen sollten im Vorfeld individuell definiert sein, damit wertvolle Informationen, wie z. B. ein Abfall des Atemminutenvolumens beim Pneumothorax, nicht verzögert erkannt werden.

Tägliche Routineröntgenaufnahmen des Thorax sind nicht zwangsläufig indiziert, auch wenn dies in einigen Arbeiten behauptet wird. Stattdessen sollte wie in anderen klinischen Bereichen jede Indikation zur radiologischen Diagnostik kritisch hinterfragt werden, um überflüssige Untersuchungen zu vermeiden. Die Bildqualität bei Röntgenthoraxaufnahmen auf der Intensivstation ist häufig unbefriedigend, sodass die Indikation nur mit entprechend konkreter Fragestellung gestellt werden sollte.

Bei ARDS-Patienten ist der routinemäßige Einsatz von Rechtsherzkathetern nicht empfehlenswert, da weder Letalität noch Morbidität günstig beeinflusst werden. Stattdessen können die katheterassoziierten Komplikationen den Patienten gefährden. Dies bedeutet allerdings nicht, dass man bei konkreten Fragestellungen auf diese Art des Monitorings verzichten sollte; ein Beispiel sind Patienten mit schwerer Herzinsuffizienz und begleitender Sepsis. Alternativen zum Rechtsherzkatheter können einerseits die Messung der zentralvenösen Sättigung sein, die einfach bestimmbar ist (über einen zentralen Venenkatheter) und invers mit dem Herzzeitvolumen korreliert (Cave: ausgeprägte Hypoxämie und Anämie), andererseits der Einsatz des PICCO-Systems, dessen Effektivität in Studien bislang nicht geprüft worden ist.

\subsubsection{Beatmungsmodi}

Die Nomenklatur der Beatmungsformen ist auf den ersten Blick sehr unübersichtlich. Im Folgenden wird daher auf die Grundprinzipien der Beatmungsformen eingegangen. 
Für spezifischere Informationen sei auf die Handbücher der jeweils verwendeten Beatmungsgeräte verwiesen.

Grunsätzlich werden 2 verschiedene Beatmungsmodi unterschieden: die kontrollierte Beatmung, bei der keine oder nur zum kleinen Teil Spontanatmung stattfindet, und die assistierte Beatmung, die eine Spontanatmung voraussetzt.

\section{Kontrollierte Beatmung}

\section{Prinzip der kontrollierten Beatmung}

Ziel der kontrollierten Beatmung ist die vollständige Übernahme der Atemarbeit und der Ersatz des Atemantriebs. Die Patienten sind dementsprechend meist so tief analgosediert, dass keine Spontanatmung vorliegt. Die Inspiration erfolgt somit durch den Respirator gemäß der vorgewählten Einstellungen, während die Exspiration ein passiver Vorgang ist, der durch die elastischen Rückstellkräfte von Lunge und Thorax sowie dem Atemwegswiderstand bestimmt wird. Ein Patient kann mit zweierlei Verfahren kontrolliert beatmet werden.

\section{Volumenkontrollierte Beatmung}

Das Prinzip der volumenkontrollierten Beatmung ist die Definition eines bestimmten Tidalvolumens, mit dem der Patient ventiliert werden soll. Über die zusätzliche Vorgabe der Atemfrequenz ergibt sich damit das applizierte Atemminutenvolumen. Der dafür erforderliche Atemwegsdruck hängt von der individuellen Compliance und Resistance $\mathrm{ab}$ und kann dementsprechend stark variieren. Alle einzustellenden Parameter sind in der Übersicht dargestellt. Die klassischen Druck- und Flusskurven einer volumenkontrollierten Beatmung sind in $\bullet$ Abb. 15.19 dargestellt.

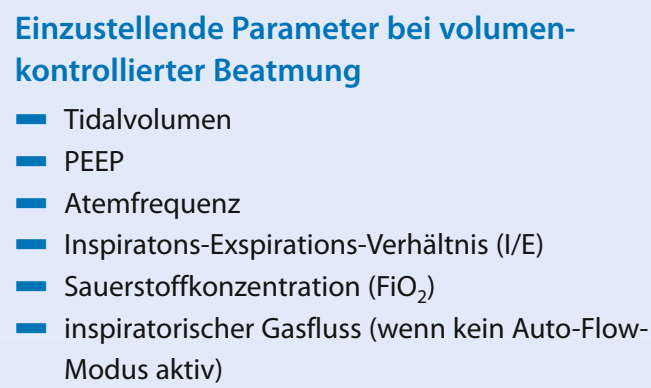

Im reinen volumenkontrollierten Modus ist keine Spontanatmung möglich. Eine Mischform zwischen kontrollierter und assistierter Beatmung ist die SIMV-Beatmung (Synchronisierte intermittierende mandatorische Ventilation). Dabei ist es möglich, neben vordefinierten kontrollierten (mandatorischen) Atemhüben, spontane Atembemühungen des Patienten zwischen den mandatorischen Atemhüben zuzulassen. Innerhalb eines vordefinierten

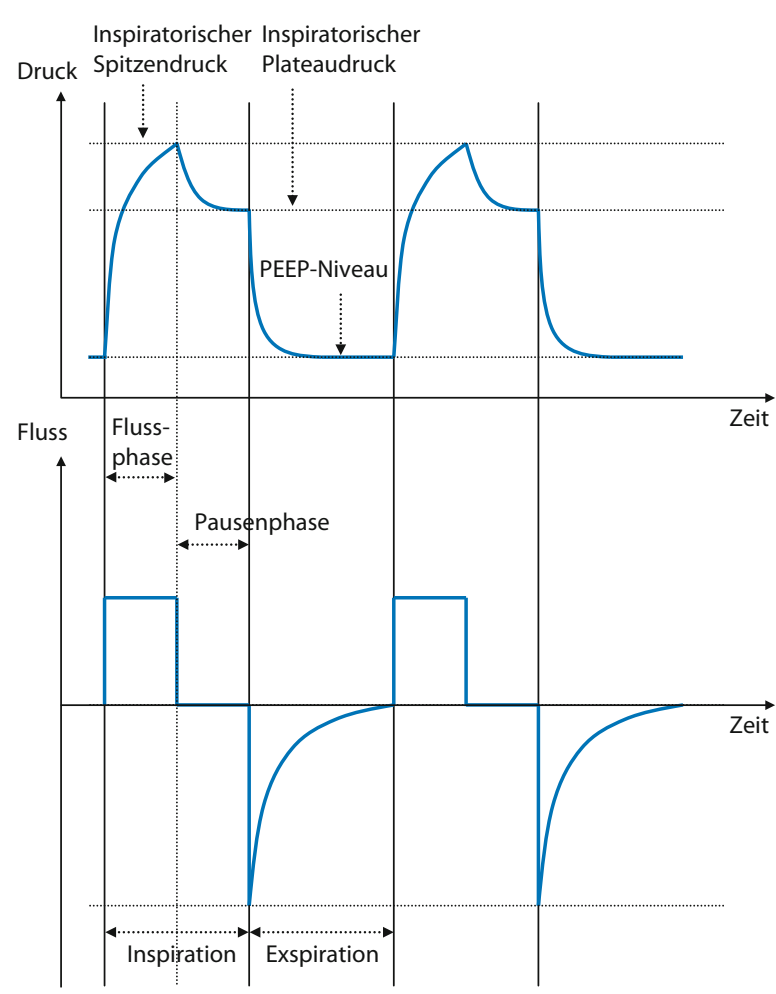

- Abb. 15.19. Volumenkontrollierte Beatmung. Der Respirator liefert Volumen bis zum Erreichen des voreingestellten Wertes für das Atemzugvolumen. Der dafür notwendige Beatmungsdruck ist von der Compliance der Lunge abhängig

Zeitfensters wird die Sponanatmung mit ASB (s. unten) unterstützt.

Die SIMV-Beatmung wurde hauptsächlich zur Respiratorentwöhnung eingesetzt; nachdem jedoch gezeigt werden konnte, dass der CPAP/ASB-Modus (s. unten) überlegen ist, kommt der SIMV-Modus seltener zur Anwendung.

\section{Druckkontrollierte Beatmung}

Im druckkontrollierten Modus wird kein bestimmtes Atemzugvolumen vordefiniert, sondern eine vom PEEP ausgehende Druckdifferenz, die über das obere Druckniveau (Pmax) bestimmt wird. Das mit dieser Druckdifferenz applizierte Atemzugvolumen ist variabel und vor allem von der Compliance des Patienten abhängig. Änderungen der Compliance, beispielsweise durch Volumenüberladung, führen bei gleicher Druckdiffierenz zu einem kleineren Tidalvolumen, dieser Beatmungsmodus ist also volumeninkonstant.

Ein sehr gebräuchlicher druckkontrollierter Modus ist der BiPAP-Modus (»biphasic positive airway pressure«). Dabei wird ein unteres (entspricht PEEP) und ein oberes Druckniveau definiert, die resultierende Druckdifferenz bestimmt das Tidalvolumen. Die Besonderheit des 


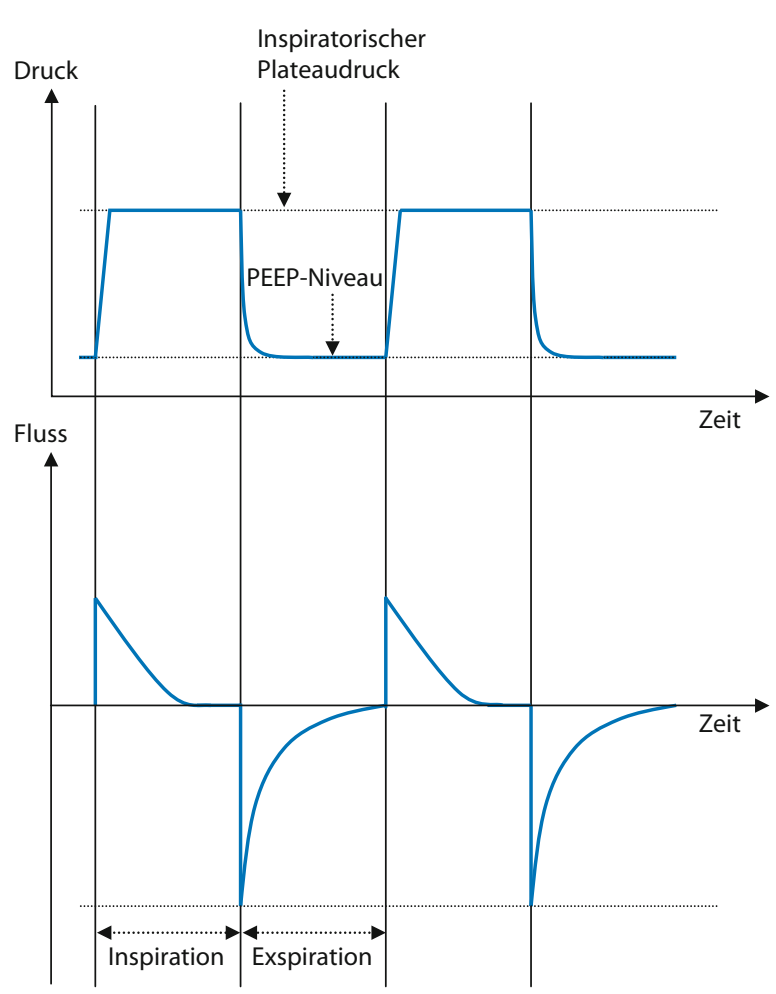

- Abb. 15.20. Druckkontrollierte Beatmung. Der Respirator liefert Volumen so lange, bis der voreingestellte maximale Beatmungsdruck erreicht ist. Das Tidalvolumen kann in Abhängigkeit von der Compliance der Lunge schwanken

BiPAP-Modus ist die Möglichkeit zur Spontanatmung auf beiden Druckniveaus unabhängig von der eingestellten Atemfrequenz. Wie unten beschrieben, kann sich eine erhaltene Spontanatmung beim akuten Lungenversagen günstig auf den Gasaustausch auswirken. Die einzustellenden Parameter im druckkontrollierten Modus sind:

- oberes Druckniveau (Pmax),

- unteres Druckniveau (PEEP),

- Atemfrequenz,

- Inspirations-Exspirations-Verhältnis (I/E),

- Sauerstoffkonzentration $\left(\mathrm{FiO}_{2}\right)$,

- zusätzlich im assistierten Modus: Trigger, Rampe und ASB.

Die typischen Druck- und Flusskurven einer druckkontrollierten Beatmung sind in • Abb. 15.20 dargestellt.

Die Mischform von druckkontrollierter Beatmung und assistierter Beatmung (Bezeichnung variiert je nach Hersteller) ermöglicht die Spontanatmung auf beiden Druckniveaus und ist mit einer Druckunterstützung (»augmented spontaneous breathing«, ASB) kombinierbar. Studien zur Evaluation dieser Beatmungsform fehlen bislang; in der Praxis sind diese Beatmungsmodi jedoch gut akzeptiert und finden breite Anwendung.

\section{Assistierte Beatmung CPAP}

Der CPAP-Modus (»continuous positive airway pressure«) wird vor allem in der nichtinvasiven Beatmung genutzt und hat im Rahmen der maschinellen Beatmung einen geringen Stellenwert. Dieser Modus setzt einen eigenen Atemantrieb voraus, die Atemwegsdrücke schwanken um das eingestellte CPAP-Niveau. Durch den permanent positiven Atemwegsdruck werden der Kollaps von Alveolen und damit das Auftreten dys- oder atelektatischer Lungenarale vermindert. Um eine CPAP-Atmung durchführen $\mathrm{zu}$ können, ist eine ausreichende Atempumpe erforderlich. Ohne eine Druckunterstützung ist dies im Rahmen der Respiratorentwöhnung in der Regel nicht möglich.

\section{CPAP/ASB}

Der Modus CPAP/ASB wird auch als "pressure support ventilation« bezeichnet und ist der gebräuchlichste Modus zur Respiratorentwöhnung. Die Druckunterstützung des Respirators erfolgt dann, wenn die Atembemühungen des Patienten erkannt werden. Dies setzt die Triggerung des Beatmungsgeräts voraus, die entweder druckgesteuert oder flussgesteuert erfolgen kann. Die Triggerschwelle kann variiert werden, wobei bedacht sein muss, dass eine zu hohe Triggerschwelle schwache Atembemühungen des Patienten nicht erkennt, eine zu empfindliche Einstellung ( $<2$ 1/min Flow-Trigger oder $<2$ mbar Drucktrigger) aber zu einer Auto-Triggerung führen kann.

Die Höhe der Druckunterstützung hängt von der individuellen Compliance von Thorax und Lunge sowie von der Kraft des Zwerchfells und der Atemhilfsmuskulatur ab. Ein zu niedrig gewählter ASB verursacht unzureichende Atemzugvolumina und Tachypnoe, also vorwiegend Totraumventilation. Daher sollte die Druckunterstützung an den aktuellen ventilatorischen Erfordernissen des Patienten adaptiert werden. Werte über $25 \mathrm{mmH}_{2} \mathrm{O}$ sind in der Regel nicht sinnvoll, da die Compliance der Lunge für eine Respiratorentwöhnung noch $\mathrm{zu}$ schlecht ist. ASB-Werte unter $10 \mathrm{cmH}_{2} \mathrm{O}$ finden in der Praxis ebenso wenig Anwendung, da ein minimaler ASB allein für den systembedingten Widerstand (Beatmungsschläuche, Tubus) erforderlich ist.

Einzustellende Parameter des CPAP/ASB-Modus sind:

- CPAP-Niveau (=PEEP),

- Triggerempfindlichkeit,

- Druckunterstützung (ASB),

- Anstiegssteilheit der Druckunterstützung (»Rampe«, möglichst kurz),

- Sauerstoffkonzentration $\left(\mathrm{FiO}_{2}\right)$.

Die Druck- und Flusskurven einer CPAP/ASB-Ventilation sind in $\bullet$ Abb. 15.21 dargestellt. 


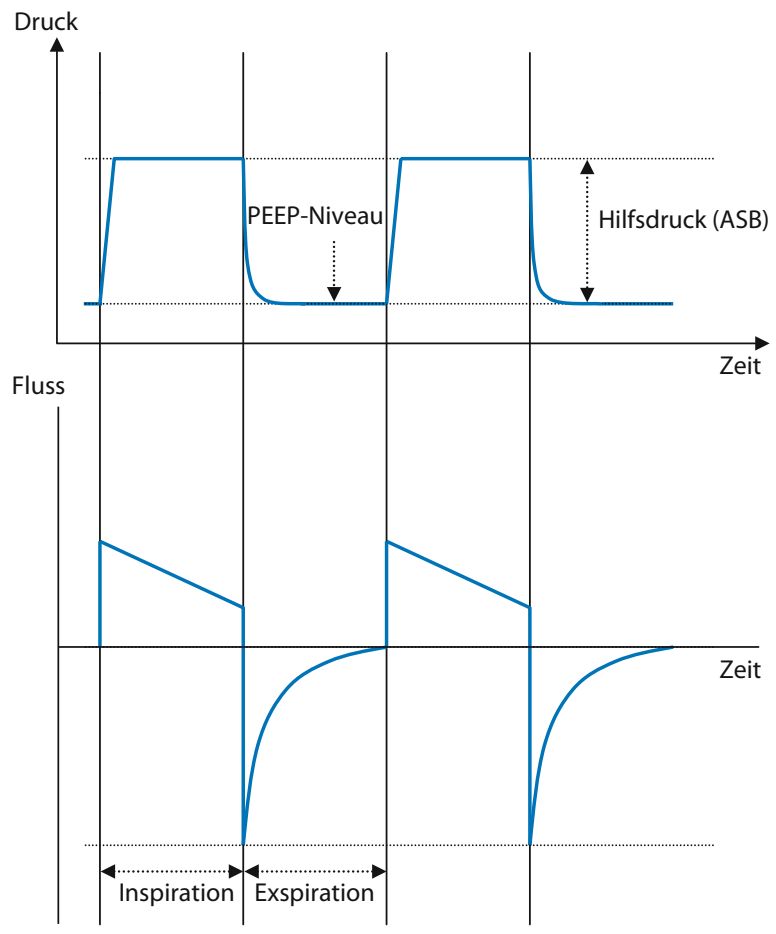

- Abb. 15.21. Spontanatmung unter CPAP/ASB

\subsubsection{Beatmung bei speziellen Erkrankungen}

Im Folgenden soll die Respiratortherapie bei 2 häufigen pulmonalen Erkrankungen auf der Intensivstation erläutert werden: das akute Lungenversagen (ALI/ARDS) und die schwere COPD bzw. der Status asthmaticus.

\section{ARDS}

Das ARDS ist eine schwere akute Erkrankung der Lunge, die nahezu immer mit der Notwendigkeit zur maschinellen Beatmung einhergeht. Aufgrund der schweren Lungenschädigungen (s. - Kap.9.1) ist die maschinelle Beatmung der ARDS-Patienten häufig eine Herausforderung. In den letzten Jahren haben sich die Kenntnisse zur Beatmung von ARDS-Patienten deutlich vermehrt. Vor allem die ARDS-Network Studie aus dem Jahr 2000 hatte großen Anteil an dem Konzept der protektiven Beatmung, welche im Folgenden vorgestellt werden soll. Im Anschluss werden supportive Konzepte der ARDSTherapie angesprochen. Weitere supportive Maßnahmen, die nicht nur für das ARDS von Bedeutung sind, werden in > Abschn. 15.4.7 erläutert.

\section{Protektive Beatmung}

Aufgrund großer multizentrischer Studien der letzten Jahre besteht Einigkeit darüber, dass ARDS-Patienten protektiv beatmet werden sollen. Aufgrund zyklischer Reund Derekrutierung von Alveolen durch hohe Tidalvolumina wird die Lunge zusätzlich zur ARDS-auslösenden Erkrankung weiter geschädigt.

Nach derzeitigem Kenntnisstand sollte daher eine Begrenzung des Plateaudruckes auf $30 \mathrm{cmH}_{2} \mathrm{O}$ und ein Tidalvolumen von $6 \mathrm{ml} / \mathrm{kg}$ ideales Körpergewicht erfolgen. Um ein ausreichendes Atemminutenvolumen zu gewährleisten, ist fast immer eine Erhöhung der Atemfrequenz erforderlich (häufig bis zu 30/min). Die im Rahmen der protektiven Beatmung auftretende Hyperkapnie kann in vielen Fällen bis zu einem resultierenden $\mathrm{pH}$ von etwa 7,15 toleriert werden (permissive Hyperkapnie). Allerdings führen hohe $\mathrm{CO}_{2}$-Werte bei einigen Patienten zu vegetativen Symptomen mit Tachykardie und Hypertonie sowie (trotz Analgosedierung) zu vermehrten Atemanstrengungen, die eine suffiziente Beatmung erschweren und die Anwendung der permissiven Hyperkapnie begrenzen können. Durch den Einsatz einer extrakorporalen Lungenunterstützung (ECLA, - Tab. 15.11) besteht keine Notwendigkeit mehr, dauerhafte $\mathrm{PCO}_{2}$-Werte über $80 \mathrm{mmHg}$ zu tolerieren. Daten zum maximal möglichen $\mathrm{PCO}_{2}$ existieren allerdings nicht.

Der Zielbereich der Oxygenierung liegt bei einer Sauerstoffsättigung von $88-95 \%$ bzw. einem $\mathrm{PO}_{2}$ von $55-80 \mathrm{mmHg}$. Das I/E-Verhältnis sollte bei 1:1 bis 1:3 liegen; der PEEP kann gemäß • Tab.15.8 dem aktuellen $\mathrm{FiO}_{2}$ zugeordnet werden, wobei es sich hier um Richtwerte handelt, die den individuellen Gegebenheiten angepasst werden müssen. Die ARDS Network Studie konnte zeigen, dass mit dem Konzept der protektiven Beatmung die Gesamtletalität auf 31\% reduziert werden konnte, im Vergleich zu 39,8\% in der konventionell beatmeten Kontrollgruppe.

Unsicherheit besteht weiterhin in Bezug auf das optimale PEEP-Niveau beim ARDS. Eine aktuelle Studie konnte unter Beatmung mit einem hohen PEEP keinen Vorteil für Letalität, beatmungsfreie Tage, Aufenthalt auf der Intensivstation, Barotrauma und Organversagen zeigen. Allerdings gab es methodische Mängel, die das Studienergebnis infrage stellen. Beispielsweise wurde nur bei einem kleinen Teil der Patienten ein RecruitmentManöver duchgeführt ( $\triangle$ Abschn. 15.4.7); die Patienten in der Gruppe mit hohem PEEP waren älter, hatten einen schlechteren Oxygenierungsindex $\left(\mathrm{P}_{\mathrm{a}} \mathrm{O}_{2} / \mathrm{FiO}_{2}\right)$ und einen etwas höheren APACHE-III-Score.

Eine generelle Empfehlung in Bezug auf das optimale PEEP-Niveau kann aufgrund vorliegender Daten nicht ausgesprochen werden und sollte bis zum Vorliegen weiterer klärender Studien eine individuelle Entscheidung bleiben.

In - Tab. 15.7 u. 15.8 sind die Maßnahmen zur Durchführung einer protektiven Beatmung aufgelistet. 


\section{Bauchlage}

Eine häufig praktizierte Maßnahme beim akuten Lungenversagen ist die Bauchlagerung. Bei $60-70 \%$ der Patienten kommt es dabei zur Verbesserung des Gasaustausches, der Sauerstoffbedarf sinkt und die Beatmung kann deeskaliert werden. Als ursächlich für diese Veränderungen werden ein erhöhtes endexspiratorisches Lungenvolumen, ein verbessertes Ventilations-Perfusions-Verhältnis und Änderungen der regionalen Ventilationsverteilung zugunsten dorsaler (abhängiger) Lungenareale angesehen.

Nachteilig ist der erhöhte Bedarf an Analgosedation, da die Bauchlage sonst schlecht toleriert wird; gelegentlich wird auch eine Muskelrelaxation notwendig. Des Weiteren kann es auch zur Kreislaufinstabilität kommen, was die Lagerungstherapie unmöglich machen kann. Beschrieben wurden auch akzidentelle Extubationen, versehentliches Entfernen von Gefäßzugängen und Atemwegsverlegungen. Häufig treten zum Teil ausgeprägte Ödeme im Gesichtsbereich auf.

In großen Studien konnte bislang kein definitiver Vorteil der Bauchlagerung in Bezug auf die Letalität gezeigt werden, es gibt jedoch Hinweise dafür, dass Patienten mit schwerster Hypoxämie $\left(\mathrm{P}_{\mathrm{a}} \mathrm{O}_{2} / \mathrm{FiO}_{2}<88 \mathrm{mmHg}\right)$ und hohem SAPS-II-Score (>49) in Bezug auf die 10-TagesLetaliät profitieren.

Neuen Daten zufolge sollte die Bauchlage möglichst innerhalb von $48 \mathrm{~h}$ nach Entwicklung eines ARDS erfolgen und ausreichend lange ( $12 \mathrm{~h}$ oder länger) aufrechterhalten werden, um einen günstigen Einfluss auf die Letaliät zu erreichen.

\section{- Tab. 15.7. Protektive Beatmung}

\begin{tabular}{l|l}
\hline Parameter & Einstellung \\
\hline Tidalvolumen & $6 \mathrm{ml} / \mathrm{kgKG}$ \\
\hline Plateaudruck max. & $30 \mathrm{cmH}_{2} \mathrm{O}$ \\
\hline Atemfrequenz & $6-35 / \mathrm{min}$ \\
\hline Permissive Hyperkapnie & $\mathrm{pH}<7,15$ \\
\hline I/E & $1: 1 \mathrm{bis} 1: 3$ \\
\hline Zieloxygenierung & $\mathrm{SpO}_{2} 88-95 \%$ bzw. $\mathrm{PO}_{2} 55-80 \mathrm{mmHg}$ \\
\hline
\end{tabular}

\section{Surfactant}

Vor allem beim ARDS und auch bei Pneumonien finden sich Störungen der Surfactantsynthese, sodass betroffene Lungenareale dys- oder atelektatisch werden können. Die Applikation von rekombinantem Surfactant bei ARDS wurde in einer großen multizentrischen Studie untersucht; ein Überlebensvorteil konnte nicht gezeigt werden. Die Subgruppenanalyse der Patienten mit primärem ARDS (Aspiration und Pneumonie) ergab jedoch einen leichten Letalitätsvorteil. Daher wird zurzeit eine PhaseIII-Studie zur weiteren Klärung der Surfactantindikation durchgeführt. Gegenwärtig kann die Surfactantgabe nicht im Rahmen der Routinetherapie empfohlen werden.

\section{NO-Beatmung}

Bei therapierefraktärem Oxygenierungversagen, seltener auch beim Rechtsherzversagen, kann eine inhalative Therapie mit Stickstoffmonoxid (NO) erwogen werden. In der pädiatrischen Intensivmedizin hat die NO-Beatmung bereits einen festen Stellenwert, während die Datenlage in der Erwachsenenmedizin bislang keine klare Empfehlung für NO zulässt. In 6 kontrollierten Studien hatte inhaliertes NO bei ARDS keinen Einfluss auf Beatmungstage oder Letalität. In den Studien übereinstimmend ist das Ansprechen auf die NO-Therapie bei ca. $60 \%$ der Patienten im Sinne einer verbesserten Oxygenierung.

Aufgrund der relativ hohen Kosten und den erforderlichen technischen Voraussetzungen ist die NO-Therapie gegenwärtig nur beim therapierefraktären ARDS als Ultima ratio empfehlenswert.

\section{High Frequency Oscillatory Ventilation (HFOV)}

Bei ARDS mit therapierefraktärer Hypoxämie kann eine Hochfrequenzoszillationsbeatmung (HFOV) erwogen werden. Die HFOV ermöglicht eine Verbesserung des Gasaustausches und eine homogenere Ventilationsverteilung. Trotz höherer mittlerer Atemwegsdrücke werden weniger inflammatorische Zytokine synthetisiert. Im Tiermodell wurden weniger histopathologische Hinweise auf einen ventilatorassoziierten Lungenschaden gesehen. Aufgrund der niedrigen Tidalvolumina kommt es kaum zum zyklischen Öffnen und Kollabieren von Alveolen, sodass ein wichtiger Faktor zur Entstehung eines ventilatorassoziierten Lungenschadens eliminiert ist.

Diese Beatmungsform kann mit herkömmlichen Respiratoren nicht durchgeführt werden, setzt also ein

- Tab. 15.8. Verhältnis $\mathrm{FiO}_{2} / \mathrm{PEEP}$ entsprechend dem Protokoll der ARDS Network Studie

\begin{tabular}{|c|c|c|c|c|c|c|c|c|c|c|c|c|c|c|c|c|c|}
\hline $\mathrm{FiO}_{2}$ & 0,3 & 0,4 & 0,4 & 0,5 & 0,5 & 0,6 & 0,7 & 0,7 & 0,7 & 0,8 & 0,9 & 0,9 & 0,9 & 1,0 & 1,0 & 1,0 & 1,0 \\
\hline $\begin{array}{l}\text { PEEP } \\
\left(\mathrm{cmH}_{2} \mathrm{O}\right)\end{array}$ & 5 & 5 & 8 & 8 & 10 & 10 & 10 & 12 & 14 & 14 & 14 & 16 & 18 & 18 & 20 & 22 & 24 \\
\hline
\end{tabular}


spezielles Beatmungsgerät für diesen Zweck voraus. In Deutschland sind keine Respiratoren zugelassen, die beide Beatmungsformen ermöglichen. Die Komplikationen der HFOV sind vor allem Kreislaufinstabilität bei volumendepletierten Patienten und Barotrauma, das durch die erhöhten Atemwegsmitteldrücke bedingt ist.

Zusammengefasst ist die HFOV ein effektives Verfahren, dass beim frühen ARDS mit schwerster Gasaustauschstörung zur Anwendung kommen kann, aber noch einer weiteren Evaluation im Rahmen klinischer Studien bedarf.

\section{Steroide}

Das persistierende ARDS ist gekennzeichnet durch anhaltende inflammatorische Veränderungen, vermehrter Kollagensynthese und Zellproliferation. Die Gabe von Steroiden in diesem Zusammenhang erfolgt unter der Vorstellung, die fibroproliferative Phase des ARDS zu verhindern bzw. abzuschwächen, um eine irreversibe Fibrose mit nachfolgender Langzeitbeatmung oder Langzeitschäden zu vermeiden.

Es existieren jedoch keine eindeutigen Daten, die eine definitive Empfehlung zur Steroidgabe zulassen. In einer aktuellen randomisierten multizentrischen Studie konnte kein Letaliätsvorteil gezeigt werden. Wurden Steroide 7 Tage nach ARDS-Beginn verabreicht, konnten mehr beatmungsfreie und schockfreie Tage dokumentiert werden und die Oxygenierung verbesserte sich. Wurden Steroide allerdings erst 2 Wochen nach ARDS-Beginn verabreicht, war eine erhöhte Sterblichkeit in der Steroidgruppe zu verzeichnen.

Der routinemäßige Einsatz von Steroiden zur Therapie des ARDS ist zum gegenwärtigen Zeitpunkt nicht zu empfehlen.

\section{Schweres Asthma bronchiale und schwere COPD}

Kennzeichnend für die schwere COPD und das schwere Asthma bronchiale ist vor allem die Erhöhung des Atemwegswiderstands, der besonders in der Exspiration eine deutliche Flussminderung hervorruft. Patienten mit einer schweren obstruktiven Ventilationsstörung werden in der Regel im Rahmen eines ventilatorischen Versagens beatmungspflichtig. Es sei vorangestellt, dass bei diesen Patienten vor Intubation und Beatmung bei fehlenden Kontraindikationen auf jeden Fall ein Versuch mit nichtinvasiver Beatmung unternommen werden sollte, da die invasive Beatmung mit einer höheren Letalität einhergeht.

Oxygenierungsprobleme bestehen meist nicht, zudem sind die Patienten an niedrige $\mathrm{PO}_{2}$-Werte adaptiert, sodass in der Regel nicht mit hohen $\mathrm{FiO}_{2}$-Werten beatmet werden muss. Chronisch hyperkapnische Patienten sollten nicht normokapnisch ventiliert werden, um eine posthyperkapnische Alkalose mit der damit verbundenen Linksverschiebung der Sauerstoffbindungskurve und möglichen zerebralen Perfusionstörungen zu vermeiden.

Die Respiratoreinstellungen müssen sich an der exspiratorischen Flusslimitierung orientieren: Die Atemfrequenz darf nicht zu hoch gewählt werden, um die Exspirationszeit nicht zu stark zu verkürzen. Eine Verlängerung des I/E-Verhältnisses kann erwogen werden, bei extremen Einstellungen $(>1: 3)$ kann die Inspirationszeit dadurch jedoch kritisch verkürzt sein.

Vorsicht ist geboten bei der Entstehung von AutoPEEP durch dynamische Überblähung (s. oben); durch zunehmende Überblähung besteht die Gefahr von Barotraumen und Kreislaufdepression. Bei therapierefraktärer Hyperkapnie ist eine extrakorporale $\mathrm{CO}_{2}$-Elimination $\mathrm{zu}$ erwägen.

\subsubsection{Entwöhnung vom Respirator (Weaning)}

Das erfolgreiche Entwöhnen vom Respirator stellt eine große Herausforderung an alle auf der Intensivstation tätigen Mitarbeiter dar. Ein allgemeingültiges, für jeden Patienten zutreffendes Konzept existiert nicht; der Entwöhnungsprozess ist immer ein sehr individueller Vorgang. Die allgemeinen Voraussetzungen, die ein erfolgreiches Weaning überhaupt erst ermöglichen, werden in der Übersicht zusammengefasst.

Man unterscheidet einfaches, verlängertes und kompliziertes Weaning. Beim einfachen Weaning kann nach Vorliegen der Ely-Kriterien (Ely et al. 1996) ein kurzer Spontanatmungsversuch von maximal $2 \mathrm{~h}$ erfolgen. Abbruchkriterien sind eine Herzfrequenz $>140 / \mathrm{min}$, eine Atemfrequenz $>35 / \mathrm{min}$, periphere Sauerstoffsättigung $<90 \%$, systolischer arterieller Blutdruck $>180 \mathrm{mmHg}$ oder $<90 \mathrm{mmHg}$ und/oder zunehmende Angst oder Agitation. Eine Extubation des Patienten kann erfolgen, wenn der Patient mehrere Minuten ohne Erreichen der Abbruchkriterien spontan atmen kann. Das genaue Zeitintervall, welches mindestens eingehalten werden sollte, ist allerdings umstritten.

Voraussetzungen zur Respiratorentwöhnung

- Ely-Kriterien:

- adäquate Oxygenierung $\left(\mathrm{PO}_{2} / \mathrm{FiO}_{2}>200 \mathrm{mmHg}\right.$ bei einem $P E E P \leq 5 \mathrm{cmH}_{2} \mathrm{O}$ )

- PEEP $\leq 5 \mathrm{cmH}_{2} \mathrm{O}$

- adäquater Husten- und Schluckreflex

- kein Vasopressorbedarf

- kein permanenter Sedationsbedarf

- Atemfrequenz/Tidalvolumen (»rapid shallow breathing index«) $<105$ 
- Therapie der Grunderkrankung

- adäquate Vigilanz und Atemantrieb

- Schmerzfreiheit und Temperatur $<38^{\circ} \mathrm{C}$

- Ausschluss einer schwerwiegenden floriden Infektion

- adäquater Säure-Base-Status (pH>7,25)

Das verlängerte Weaning tritt häufig nach einer längeren Beatmungsdauer (ca. 7-14 Tage) auf. Der Entwöhnunsgprozess ist jedoch meist kurz und setzt nicht unbedingt eine Tracheotomie voraus. Langzeitbeatmete Patienten sollten zum Weaning allerdings tracheotomiert sein, da in diesem Fall der Entwöhnungsprozess über einen orotrachealen oder nasotrachealen Tubus selten möglich ist.

Wenn ein Patient die in der Übersicht genannten Punkte erfüllt, sollte ein Spontanatmungsversuch erfolgen. Dies geschieht meistens über einen CPAP-Modus in Kombination mit inspiratorischer Druckunterstützung (ASB). Initial sind meist hohe Unterstützungsdrücke nötig, da die Atemmuskulatur noch keine dauerhaft ausreichende Ventilation aufrechterhalten kann. Des Weiteren werden die Unterstützungsdrücke sukzessive reduziert, bis eine Spontanatmung ohne Druckunterstützung möglich scheint. Es sei an diesem Punkt daran erinnert, dass das Schlauchsystem und der Tubus bzw. die Trachealkanüle selbst einen Atemwegswiderstand darstellen, der bei der Wahl des Unterstützungsdrucks berücksichtigt werden muss. Um allein diesen Widerstand zu kompensieren ist je nach Tubusgröße eine Druckunterstützung von 5-10 $\mathrm{cmH}_{2} \mathrm{O}$ erforderlich. Nach kurzer Beatmungszeit kann der Tubus meist zu diesem Zeitpunkt entfernt werden. Nach Langzeitbeatmung sind die Spontanatmungsphasen ohne Druckunterstützung zunächst kurz und müssen mehrmals täglich wiederholt werden, um einen Trainingseffekt auf die Atemmuskulatur zu erwirken. Nach 24 h Spontanatmung ohne Respiratorunterstützung können die Patienten zumeist extubiert (bzw. bei Tracheotomie dekanüliert) werden, sodass nun auch wieder eine selbstständige Bronchialtoilette möglich ist.

Patienten, die mit dem oben beschriebenen Verfahren nicht vom Respirator entwöhnt werden können, gelten als schwer zu entwöhnende Patienten (kompliziertes Weaning). Bei hyperkapnische Patienten kann nach Extubation eine nichtinvasive Ventilation versucht werden, bei komplexeren Problemen muss ein multifaktorieller Ansatz mit Optimierung des Ernährungsstatus, Reduktion der Sputummenge, Totraumverkleinerung (Tracheotomie) und ggf. auch Anxiolyse erfolgen. Bei ausgeprägter »critical illness «-Neuromyopathie kann auch ein Zuwarten unter intensiver physiotherapeutischer Behandlung bis zur allmählichen Besserung indiziert sein. Bei persis- tierender Respiratorabhängigkeit sollte eine Verlegung in ein Weaning-Zentrum erwogen werden.

In der Vergangenheit konnte gezeigt werden, dass Weaningprotokolle oder in Respiratoren erhältliche Weaningprogramme zum Teil zu einem besseren Entwöhnungserfolg führen können als ein individuelles, nicht klar strukturiertes Vorgehen. Dies gilt vor allem auf Intensivstationen mit geringer Expertise in Bezug auf die Respiratorentwöhnung. In erfahrenen Teams konnte kein Vorteil eines protokollbasierten Vorgehens gegenüber einer individuell durch den Behandler festgelegten Weaningstrategie nachgewiesen werden.

Verschlechtert sich ein Patient nach erfolgter Extubation, sodass er erneut beatmet werden muss, handelt es sich um ein Postextubationsversagen. Über die in der Übersicht aufgeführten Kriterien hinaus existieren keine Prädiktoren für ein Postextubationsversagen. Patienten mit einem reinen Ventilationsversagen sollten unter Berücksichtigung der Kontraindikationen nichtinvasiv beatmet werden. Bei zunehmender respiratorischer Azidose als Ausdruck eines Therapieveragens ist eine endotracheale Intubation unumgänglich. Bei Patienten mit einem Oxygenierungsversagen sollte ein Therapieversuch mit einer CPAP-Maske erfolgen, nach Ausbleiben einer Besserung ist auch hier eine invasive Beatmung indiziert. Bei bekanntem Erregerspektrum kann eine Antibiotikaprophylaxe vor Reintubation erwogen werden, um einer ventilatorassoziierten Pneumonie vorzubeugen, da es in der Regel zu Erregeraspirationen durch Reintubation kommt.

\subsubsection{Supportive Konzepte}

\section{Analgosedation}

Eine Analgosedation ist bei maschineller Beatmung nahezu immer erforderlich. Ein Ramsay-Score (• Tab. 15.9) von 3-4 ist bei unkomplizierter Beatmung ausreichend. Eine tiefere Sedation ist in Ausnahmefällen nötig (Bauchlage, hohe Beatmungsdrücke, niedrige Atemzugsvolumina oder permissive Hyperkapnie), da die Spontanatmung des Patienten nicht um jeden Preis unterdrückt werden muss. Es gibt zunehmend Hinweise dafür, dass die Erhaltung der Spontanatmung die Ventilation zwerchfellnaher Lungenanteile und damit den Gasaustausch verbessert. Des Weiteren führt eine übermäßige Sedationstiefe $\mathrm{zu}$ einer unnötig langen Beatmungsdauer (v. a. bei Patienten mit höhergradiger Leber- oder Niereninsuffizienz) und kann zur Entstehung einer »critical illness «-Neuropathie und Myopathie beitragen. Das tägliche Pausieren der Analgosedation kann dementsprechend die Beatmungstage und den Aufenthalt auf der Intensivstation verkürzen, wie dies im Jahre 2000 in einer prospektiven Studie an 128 Patienten eindrucksvoll gezeigt werden konnte (Kress et al. 2000). 


\begin{tabular}{l|l|l|}
\hline $\begin{array}{l}\text { - Tab. 15.9. } \text { Ramsay-Score } \\
\text { Score }\end{array}$ & Beschreibung des Patienten & Beurteilung \\
\hline 0 & Wach,orientiert & Wach \\
\hline 1 & Agitiert, unruhig, ängstlich & Zu flach \\
\hline 2 & Wach, kooperativ, beatmungstolerant & Adäquat \\
\hline 3 & Schlafend, aber kooperativ (öffnet Augen auf laute Ansprache oder Berührung) & Adäquat \\
\hline 4 & $\begin{array}{l}\text { Tiefe Sedierung (öffnet Augen auf laute Ansprache oder Berührung nicht, aber prompte Reaktion auf } \\
\text { Schmerzreize) }\end{array}$ & Adäquat \\
\hline 5 & Narkose (träge Reaktion auf Schmerzreize) & Tief \\
\hline 6 & Tiefes Koma (keine Reaktion auf Schmerzreize) & Zu tief
\end{tabular}

\section{Frühe Tracheotomie}

Bei Patienten, die voraussichtlich länger als 1 Woche beatmet bleiben (z. B. ARDS oder schwere Pneumonie), ist eine frühzeitige Tracheotomie durchzuführen. Im Vergleich zu einer prolongierten translaryngealen Intubation sind die Letalität, die Rate von ventilatorassoziierten Pneumonien, die Beatmungstage und der Intensivaufenthalt deutlich und signifikant geringer. Methode der Wahl ist in vielen Zentren heute die perkutane Punktionstracheotomie.

\section{Recruitment}

Die pathophysiologischen Veränderungen beim akuten Lungenversagen beinhalten unter anderem einen Kollaps von Alveolen. Diese regionalen Atelektasen sind inhomogen in der Lunge verteilt und vor allem in den abhängigen Anteilen zu finden. Diese Veränderungen (»baby lung concept«) führen zu Störungen des VentilationsPerfusions-Verhältnisses und zu intrapulmonalen Shunts. Die Folge davon ist eine schwere Hypoxämie, die oftmals sauerstoffrefraktär ist.

Die innerhalb eines Beatmungszyklus sich öffnenden und wieder kollabierenden Alveolen tragen wesentlich zum oben erwähnten ventilatorassoziierten Lungenschaden bei. Ziel eines Recruitmentmanövers soll es sein, Alveolen durch erhöhte intrapulmonale Drücke zu eröffnen und durch einen erhöhten PEEP (theoretisch oberhalb des unteren Inflektionspunkts) offen zu halten. In diesem Zusammenhang sei darauf hingewiesen, dass es bei jedem Absaugmanöver zur Derekrutierung von Alveolen kommen kann. Die gelegentliche Verschlechterung der Oxygenierung nach bronchoskopischem Absaugen ist unter anderem darauf zurückzuführen.

Es gibt verschiedene Methoden, ein RecruitmentManöver durchzuführen:

- Das obere Druckniveau wird angehoben und für mehrere Beatmungszyklen mit einem Pmax z. B. von $40-50 \mathrm{cmH}_{2} \mathrm{O}$ oder mehr beatmet.
- Der Beatmungszyklus wird auf dem Druckniveau der Inspiration für mehrere Sekunden angehalten. Zuvor wird der PEEP über das zuletzt bestehende Niveau angehoben. Kommt es während des »inspiration hold «Manövers zu einer Verbesserung der Oxygenierung wird der PEEP so lange reduziert, bis es $\mathrm{zu}$ einer Verschlechterung der Oxygenierung kommt (am unteren Inflexionspunkt). Danach wird ein erneutes Rekrutierungsmanöver durchgeführt, bei dem der PEEP oberhalb des unteren Inflexionspunkts eingestellt und belassen wird.

- Der PEEP wird schrittweise um $5 \mathrm{cmH}_{2} \mathrm{O}$ für jeweils 2 min erhöht, beginnend bei $20 \mathrm{cmH}_{2} \mathrm{O}$ bis zu maximal $45 \mathrm{cmH}_{2} \mathrm{O}$ bei fixer Druckdifferenz von $15 \mathrm{cmH}_{2} \mathrm{O}$ bis zum Erreichen eines $\mathrm{P}_{\mathrm{a}} \mathrm{O}_{2}+\mathrm{PCO}_{2}>400 \mathrm{mmHg}$ bei einem $\mathrm{FiO}_{2}$ von 1,0 .

Die häufigste Nebenwirkung eines Rekrutierungsmanövers ist eine passagere arterielle Hypotension, die vor allem bei volumendepletierten Patienten auftritt. Ursächlich ist wie bereits oben angeführt ein verminderter venöser Rückfluss durch den erzeugten hohen intrathorakalen Druck mit konsekutivem Abfall des Herzzeitvolumens und damit des arteriellen Blutdrucks. Eine Katecholamintherapie ist nicht erforderlich, eine Flüssigkeitszufuhr kann sinnvoll sein. Nach Beenden des Rekrutierungsmanövers erholt sich der Blutdruck rasch.

\section{Flüssigkeitsmanagement}

Das optimale Flüssigkeitsmanagement beim ARDS ist nicht abschließend geklärt. Negativbilanzierung durch forcierte Diurese und Flüssigkeitsrestriktion, eventuell auch in Kombination mit einer Albumingabe, können den Gasaustausch verbessern, andererseits ein extrapulmonales Organversagen fördern. Eine großzügige Flüssigkeitszufuhr wiederum kann aufgrund der vermehrten kapillären Permeabiltät den Gasaustausch beeinträchtigen. 
Beim akuten Lungenversagen (ALI/ARDS) ist ein geringer Anstieg des pulmonalarteriellen Verschlussdrucks mit einem überproportionalen Anstieg des extravaskulären Lungenwassers vergesellschaftet. Aktuellen Daten zufolge hat ein restriktives Flüssigkeitsregime einen verbesserten Oxygenierungsindex und mehr beatmungsfreie Tage zur Folge, ohne dass vermehrt ein extrapulmonales Organversagen auftritt. Es ergibt sich dadurch jedoch kein signifikanter Überlebenvorteil, sodass dieses Konzept nicht als klar überlegen anzusehen ist.

\section{Erhaltene Spontanatmung}

Eine Unterdrückung der Spontanatmung des Patienten ist nicht unbedingt erforderlich. Die erhaltene Spontanatmung führt zur verbesserten Ventilation dystelektatischer, vor allem zwerchfellnaher Lungenareale und damit zu einem günstigeren Ventilations-PerfusionsVerhältnis.

Unter protektiver Beatmung finden sich bei (permissiver) Hyperkapnie häufig spontane Atembemühungen des Patienten. Daher sollte mit einem entsprechenden Beatmungsmodus (z. B. BiPAP) ventiliert weden, ggf. auch mit einer Druckunterstützung. Eine Vertiefung der Analgosedation ist jedoch gelegentlich bei hohen Beatmungsdrücken und ausgeprägter Hyperkapnie unerlässlich. Zu den in der Beatmungsmedizin gebräuchlichen Abkürzungen vergleiche •Tab. 15.3.

\subsubsection{Lungenersatzverfahren}

\section{M.M. Hoeper}

Nach wie vor kommt es im klinischen Alltag zu Situationen, in denen die oben beschriebenen Beatmungsverfahren nicht ausreichen, um einen ausreichenden Gastaustausch zu gewährleisten, oder in denen ein kombiniertes kardiopulmonales Versagen vorliegt, das mit konventionellen Methoden nicht beherrschbar ist. In diesen Fällen können unter bestimmten Umständen extrakorporale Lungen- oder Herz-Lungen-Ersatzverfahren zum Einsatz kommen. Der folgende Abschnitt gibt einen Überblick über die technischen Möglichkeiten und potenzielle klinische Indikationen, aber auch über die Grenzen dieser Verfahren.

\section{Terminologie}

Die Terminologie der extrakorporalen Lungenersatzverfahren ist kompliziert und uneinheitlich. - Tab. 15.10 fasst die derzeit gebräuchlichen Begriffe zusammen, wobei einige dieser Begriffe in der Literatur unterschiedlich verwendet werden.

\begin{tabular}{|l|l|}
\hline $\begin{array}{l}\text { - Tab. 15.10. Terminologie extrakorporaler Lungenersatz- } \\
\text { verfahren }\end{array}$ \\
\hline ECMO & $\begin{array}{l}\text { Extrakorporale Membranoxygenierung } \\
\text { (»extracorporeal membran oxygenation«) }\end{array}$ \\
\hline $\mathrm{ECLA}$ & $\begin{array}{l}\text { Extrakorporale Lungenunterstützung } \\
\text { (»extracorporeal lung assist«) }\end{array}$ \\
\hline $\mathrm{ECLS}$ & $\begin{array}{l}\text { Extrakorporale Lungenunterstützung } \\
\text { (»extracorporeal lung support«) }\end{array}$ \\
\hline $\mathrm{iLA}$ & $\begin{array}{l}\text { Interventionelle Lungenunterstützung } \\
\text { (»interventional lung assist«) }\end{array}$ \\
\hline $\mathrm{ECCO}_{2}-\mathrm{R}$ & $\begin{array}{l}\text { Extrakorporale } \mathrm{CO}_{2} \text {-Elimination } \\
\text { (»extracorporeal } \mathrm{CO}_{2} \text { removal«) }\end{array}$ \\
\hline
\end{tabular}

\section{Historie und gegenwärtige Entwicklungen}

Experimente zur extrakorporalen Perfusion isolierter Organe wurden bereits Ende des 19. Jahrhunderts durchgeführt, aber erst in den 50er-Jahren des 20. Jahrhunderts standen Herz-Lungen-Maschinen zur Verfügung, mit denen Operationen am offenen Herzen und an den großen Gefäßen möglich wurden. Hill und Mitarbeiter beschrieben 1972 erstmalig den erfolgreichen Einsatz der extrakorporalen Membranoxygenierung (ECMO) bei einem 24-jährigen Patienten mit Lungenversagen nach Polytrauma. 4 Jahre später erfolgte die erste Fallbeschreibung eines erfolgreichen ECMO-Einsatzes bei einem Neugeborenen. Der diesen Berichten folgende Enthusiasmus wurde rasch und nachhaltig gedämpft, nachdem 1974 eine randomisierte Multizenterstudie zum Einsatz der ECMO bei erwachsenen ARDS-Patienten katastrophale Ergebnisse brachte. Die Sterblichkeit betrug sowohl in der konventionellen Therapiegruppe als auch in der ECMOGruppe ca. $90 \%$.

Trotz dieser enttäuschenden Daten beschäftigte sich insbesondere die Arbeitsgruppe von Gattinoni weiterhin mit extrakorporalen Lungenersatzverfahren, jetzt zunehmend unter dem Gesichtspunkt der Lungenprotektion. Bei diesem Ansatz wurden die Lungen durch extrem niedrige Atemfrequenzen und Zugvolumina weitgehend ruhig gestellt. Oxygenierung und $\mathrm{CO}_{2}$-Elimination wurden konzeptionell voneinander getrennt. Die Sauerstoffversorgung erfolgte weitgehend über den Respirator mittels hypopnoeischer/apnoeischer Oxygenierung, also per Diffusion, während die $\mathrm{CO}_{2}$-Elimination vorwiegend extrakorporal erfolgte, sodass dieses Verfahren auch extrakorporale $\mathrm{CO}_{2}$-Elimination (extracorporeal carbon dioxide removal, $\mathrm{ECCO}_{2}-\mathrm{R}$ ) genannt wurde.

Da für dieses Verfahren geringere Blutflüsse erforderlich waren als für eine vollständige kardiopulmonale Unterstützung, konnte man anstelle eines venoarteriellen Verfahrens auf eine weniger invasive venovenöse Zirkula- 
tion zurückgreifen. Erste unkontrollierte klinische Studien mit diesem Verfahren bei erwachsenen ARDS-Patienten berichteten Überlebensraten von etwa 50\%. Daraufhin wurde Anfang der 90er-Jahre erneut eine kontrollierte klinische Studie aufgelegt, in der erwachsene ARDS-Patienten entweder mit konventioneller Beatmung oder mit protektiver Ventilation und extrakorporaler $\mathrm{CO}_{2}$-Elimination behandelt wurden. In dieser Studie konnte wiederum kein signifikanter Überlebensvorteil der extrakorporalen Therapie gegenüber der konventionellen Beatmung gezeigt werden, auch wenn die Überlebensraten mit 33\% bzw. $42 \%$ jetzt insgesamt deutlich besser waren als in der oben beschriebenen ECMO-Studie.

Damit konnten die beiden bisher einzigen kontrollierten Therapiestudien zum Einsatz extrakorporaler Lungenersatzverfahren keinen Überlebensvorteil der Interventionsgruppe zeigen. Zum Teil wurde dies auf technische Unzulänglichkeiten der Systeme zurückgeführt. Insbesondere Blutungskomplikationen stellten ein großes Problem dar, zumal die seinerzeit eingesetzten Systeme eine kontinuierliche Antikoagulation erforderten. Die Entwicklung heparinbeschichteter Systeme hat die Notwendigkeit einer strengen Antikoagulation und damit die Gefahr von Blutungskomplikationen mittlerweile reduziert. Dennoch ist der Einsatz der ECMO in der Therapie des ARDS in den letzten Jahren kontinuierlich zurückgegangen, was zum Teil auf die ernüchternden Studienergebnisse, zum Teil aber auch auf verbesserte Beatmungstechniken und supportive Maßnahmen wie den Einsatz der Bauchlage sowie der NO-Beatmung zurückzuführen ist.

Dennoch bleibt der Einsatz extrakorporaler Lungenunterstützungsverfahren in der Intensivtherapie des ARDS ein aktuelles Thema. Zahlreiche experimentelle und klinische Studie haben übereinstimmend gezeigt, dass hohe Atemzugvolumina und hohe Druckamplituden die Lungen schädigen und ein ARDS sowie die systemische Inflammation unterhalten können. Gleichzeitig konnten mehrere randomisierte, kontrollierte Studie belegen, dass lungenprotektive Beatmungsstrategien mit hohen PEEPWerten, niedrigen Druckamplituden und entsprechend niedrigen Atemzugvolumina einen Überlebensvorteil bringen. Diese modernen Beatmungskonzepte werden im klinischen Alltag jedoch häufig durch die daraus resultierende Hyperkapnie limitiert.

Bislang ist nicht abschließend geklärt, ob eine Hyperkapnie per se ein klinisches Problem darstellt und leichte Hyperkapnien mit $\mathrm{P}_{\mathrm{a}} \mathrm{CO}_{2}$-Werten bis ca. $60 \mathrm{mmHg}$ gelten in aller Regel als unproblematisch. Ausgeprägtere Hyperkapnien werden jedoch von nicht adaptierten Patienten trotz Analgosedierung nur schlecht toleriert und führen zu Unruhe, vegetativen Symptomen wie Tachykardie und Hypertonus sowie unter Umständen $\mathrm{zu}$ einer schweren respiratorischen Azidose. In solchen Fällen kann die protektive Beatmungsstrategie möglicherweise nur durch den Einsatz einer unverhältnismäßig tiefen Analgosedierung fortgesetzt werden, die jedoch ihrerseits mit zahlreichen Problemen und Komplikationen behaftet ist ( Abschn. 15.4.7).

Vor diesem Hintergrund wurde in den letzten Jahren ein pumpenloses arteriovenöses Lungenunterstützungsverfahren entwickelt, das unter anderem unter den Begriffen ECLA (»extracorporeal lung assist»), iLA (»interventional lung assist«) oder ECLS (»extracorporeal lung support «) bekannt geworden ist. Dieses Verfahren dient ebenso wie die $\mathrm{ECCO}_{2}$-R vorwiegend der $\mathrm{CO}_{2}$-Elimination und wird vor allem in Europa in zunehmendem Maße eingesetzt, obgleich hierzu noch keine kontrollierten Studien vorliegen.

\section{Venoarterielle Lungenunterstützung}

Die venoarterielle Lungenunterstützung stellt das klassische Verfahren des extrakorporalen Lungen- bzw. HerzLungen-Ersatzes dar und wird häufig mit der extrakorporalen Membranoxygenierung (ECMO) gleichgesetzt. Der apparative Aufwand ist erheblich und die Zirkulation wird dabei partiell oder vollständig von einer extrakorporal arbeitenden Pumpe übernommen. Die venöse Blutentnahme erfolgt über großlumige Kanülen (ca. $28 \mathrm{G}$ ), die in der Regel über die Femoralvenen, gelegentlich aber auch über die Vv. jugulares oder Vv. subclaviae eingeführt und idealerweise bis an oder in den rechten Vorhof vorgeschoben werden, um einen ausreichenden Blutfluss zu gewährleisten. Die arterielle Kanülierung erfolgt fast ausnahmslos über eine Femoralarterie, wobei gelegentlich auch eine direkte Kanülierung der Aorta ascendens via Sternotomie erfolgt.

Während diese Form der ECMO historisch bei Patienten mit ARDS eingesetzt wurde, kommt sie heutzutage im Wesentlichen bei Patienten mit kardialem oder kardiopulmonalem Versagen zum Einsatz. Eine Indikation ist beispielsweise die schwere akute Myokarditis mit kardialem Pumpversagen, bei dem die ECMO als überbrückende Maßnahme eingesetzt wird. Beim reinen Lungenversagen wird heute eher auf die unten beschriebenen venovenösen oder arteriovenösen Verfahren zurückgegriffen.

Bei Ausfall der Herzfunktion übernimmt die ECMO vollständig die Pumpfunktion und tritt funktionell anstelle des Herzens. Der Blutfluss bleibt somit im Wesentlichen "physiologisch«. Bei vollständig oder partiell erhaltener kardialer Funktion sind die Strömungsverhältnisse im Bereich der Aorta und der großen Arterien unter Umständen kompliziert, je nachdem wo die Spitze der arteriellen Kanüle positioniert ist bzw. wo die Durchmischung des kardialen Blutflusses mit dem Echofluss erfolgt. Bei schwer gestörtem pulmonalem Gasaustausch kann es unter diesen Bedingungen trotz ausreichender ECMO-Funktion zu einer Versorgung des zentralen 
Nervensystems mit sauerstoffarmem Blut kommen. Daher ist es gerade in diesen Fällen wichtig, die arterielle Oxygenierung an Stellen zu überwachen, die das zentrale Nervensystem repräsentieren, wie z.B. die rechte Radialarterie.

Die pumpenabhängige venoarterielle ECMO ist ein ausgesprochen komplikationsträchtiges Verfahren. $\mathrm{Zu}$ den wichtigsten Komplikationen gehören vor allem Blutungen, aber auch Thrombosen der katheterführenden Gefäße sowie Infektionen. Darüber hinaus kommt es bereits nach wenigen Tagen des ECMO-Einsatzes durch Aktivierung von Leukozyten, Komplement, Cytokinen und Gerinnungsfaktoren sowie durch eine Hämolyse fast regelhaft zum Bild einer systemischen Inflammation, das in der Regel in einem Multiorgandysfunktionssyndrom endet. Gerade diese Komplikation macht den längeren Einsatz der ECMO ( $>5-7$ Tage) in vielen Fällen unmöglich und ist sicher für die weiterhin schlechten Ergebnisse dieses Verfahrens entscheidend mitverantwortlich.

In jüngeren Publikationen wird die Überlebensrate von ECMO-behandelten Patienten zwar mit bis zu 50\% angegeben, man darf aber durchaus davon ausgehen, dass die Resultate vieler nichtpublizierter Fallserien auch heute noch deutlich schlechter sein dürften, zumal die venoarterielle ECMO zur Therapie des isolierten Lungenversagens im deutschsprachigen Raum derzeit kaum zum Einsatz kommt.

\section{Venovenöse Lungenunterstützung}

Wenn man sich heute bei schwerem Lungenversagen mit hochgradig gestörter Oxygenierung für ein extrakorporales Lungenersatzverfahren entscheidet, bietet sich der venovenöse Lungenersatz an. Ähnlich wie bei venoarteriellen Verfahren ist auch hier eine Pumpe zur Aufrechterhaltung des Blutflusses durch den Oxygenator erforderlich. Da dieses Verfahren jedoch bei Patienten mit intakter kardialer Funktion eingesetzt wird, ist eine vollständige Übernahme der Herzfunktion nicht erforderlich, sodass Flussraten von 2-31/min in der Regel ausreichen, um eine ausreichende arterielle Oxygenierung sicherzustellen. Somit kann der apparative Aufwand deutlich geringer gestaltet werden als bei den venoarteriellen Verfahren und auch die Gefahr systemischer Komplikationen ist geringer.

Die venösen Kanülen werden zumeist über die Femoral- und Jugularvenen eingeführt, wobei auch ein bifemoraler Zugang gewählt werden kann. Die Platzierung der Kanülenspitzen ist entscheidend für einen ausreichenden Blutfluss. Idealerweise wird die drainierende Kanüle am Eingang des rechten Vorhofs platziert, während man die zuführende Kanüle proximal im rechten Vorhof einsetzt. Eine zu tiefe bzw. überlappende Positionierung der Kanülen führt zu Rezirkulationsproblemen und damit zu einer verminderten Effizienz des Verfahrens. Bei korrekter Positionierung der Kanülen und ausreichendem Blutfluss über das System wird eine hohe Sauerstoffsättigung in der Pulmonalarterie erzielt, sodass selbst bei weitgehend oder vollständig aufgehobenem pulmonalem Gasaustausch eine ausreichende arterielle Oxygenierung in allen Abschnitten der systemischen Zirkulation inklusive der Koronararterien und des zentralen Nervensystems sichergestellt wird.

\section{Arteriovenöse Lungenerstützung}

Dieses Verfahren wird derzeit in Deutschland am häufigsten zur extrakorporalen Lungenunterstützung eingesetzt. Das Grundprinzip ist die funktionelle Trennung des pulmonalen Gasaustauschs in Oxygenierung und Ventilation bzw. $\mathrm{CO}_{2}$-Elimination, wobei das extrakorporale System im Wesentlichen die $\mathrm{CO}_{2}$-Elimination übernimmt, während die Oxygenierung zum größten Teil weiter über die Lungen erfolgt. Somit ist dieses Verfahren nur sinnvoll, wenn eine hinreichende Oxygenierung über die Lungen gewährleistet ist. Hinter dem Konzept der extrakorporalen $\mathrm{CO}_{2}$-Elimination verbirgt sich der Wunsch nach einer konsequenten protektiven Beatmungsstrategie mit möglichst niedrigen Druckamplituden und Tidalvolumina, der im klinischen Alltag durch die resultierende Hyperkapnie häufig Grenzen gesetzt sind. Allerdings wurde auch diese Strategie bislang nicht in prospektiven, kontrollierten Studien hinsichtlich einer tatsächlichen Verbesserung der Behandlungsergebnisse evaluiert.

Die arteriovenöse Lungenunterstützung erfolgt ohne externe Pumpe mit dem arteriovenösen Druckgradienten als treibende Kraft für den Blutfluss. Fast ausschließlich wird der bifemorale Zugang verwendet. Die Kanülen haben mit 13-17 G einen deutlich geringeren Durchmesser als die klassischen ECMO-Kanülen (ca. 28 G). Dennoch weisen sie zusammen mit den verwendeten Membranen und Leitungen einen sehr niedrigen Eigenwiderstand auf, sodass auch bei längerer Anwendung keine Pumpen erforderlich werden. Voraussetzung für den erforderlichen Blutfluss von ca. $1000-2500 \mathrm{ml} / \mathrm{min}$ ist allerdings ein ausreichender mittlerer systemischer Blutdruck von mindestens $90 \mathrm{mmHg}$. Der Aufbau des Systems ist in • Abb. 15.22 dargestellt.

Der Blutfluss über das System wird mit einem speziellen Monitor basierend auf der Doppler-Technologie fortlaufend kontrolliert, um eine Thrombosierung des Systems frühzeitig $\mathrm{zu}$ erkennen. Die $\mathrm{CO}_{2}$-Elimination hängt im Wesentlichen von dem Sauerstofffluss über die Membran ab und kann über diesen Fluss einfach gesteuert werden. $\mathrm{Zu}$ Therapiebeginn ist darauf zu achten, den Sauerstofffluss langsam zu steigern um einen abrupten $\mathrm{CO}_{2}$-Abfall mit entsprechenden Disäquilibriumsproblemen zu vermeiden. 
Die Tatsache, dass dieses Verfahren, das auch »interventional lung assist « (iLA) genannt wird, vorwiegend zur $\mathrm{CO}_{2}$-Elimination geeignet ist, hängt mit den unterschiedlichen physikalischen Eigenschaften von Sauerstoff und Kohlendioxid im Blut zusammen. Kohlendioxid wird vorwiegend in gelöster Form transportiert und kann daher während einer Passage durch die Membran praktisch vollständig eliminiert werden, sodass selbst bei ausgeprägter Hyperkapnie binnen kurzer Zeit ein Äquilibrium im Bereich eukapnischer Werte entsteht. Sauerstoff hingegen ist vorwiegend an das Hämoglobin der Erythrozyten gebunden und zwar in Form einer charakteristischen Dissoziationskurve, die dazu führt, dass selbst bei niedrigen arteriellen Sauerstoffpartialdrücken (z. B. $40 \mathrm{mmHg}$ ) immer noch verhältnismäßig hohe Sauerstoffsättigungen (bei $40 \mathrm{mmHg} 70-80 \%$ ) bestehen. Der im Blut gelöste Anteil hingegen ist verschwindend gering. Somit lässt sich der Sauerstoffgehalt des Blutes durch die Membran nur um ca. 20-30\% erhöhen und dies auch nur in dem Anteil des gesamten Blutflusses, der durch die Membran fließt (ca. 10-20\%).

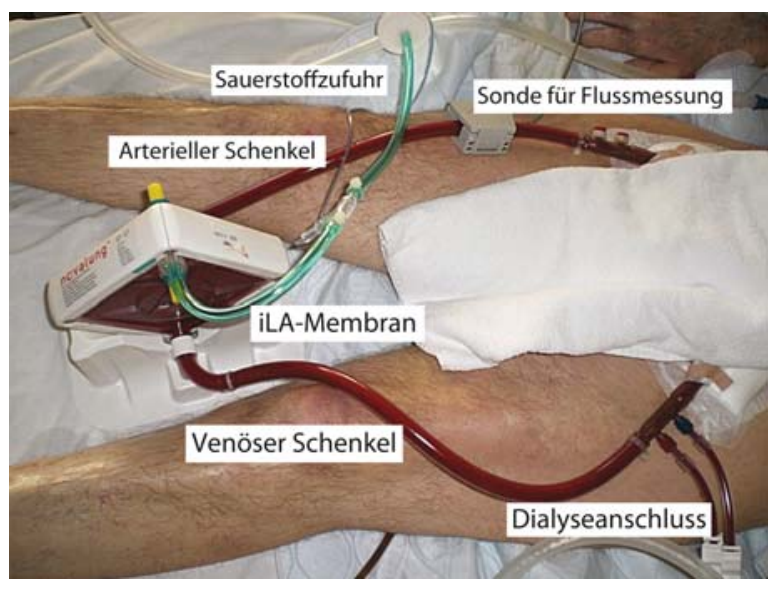

- Abb. 15.22. Klinischer Einsatz einer pumpenfreien arteriovenösen Lungenunterstützung. Das Blut fließt über den femoralarteriellen Schenkel des Systems durch die iLA-Membran (»interventional lung assist«-Membran) und von dort über den femoralvenösen Schenkel zurück in den Organismus. An dem arteriellen Schenkel ist eine Flusssonde angebracht. Über den venösen Schenkel kann bei Bedarf ein Dialysegerät angeschlossen werden
Zahlreiche Kasuistiken und Fallserien haben mittlerweile gezeigt, dass die iLA bei Patienten mit akutem oder chronischem Lungenversagen erfolgreich eingesetzt werden kann, und dies zum Teil über mehrere Wochen, was mit der konventionellen ECMO praktisch nicht möglich ist. Bein und Mitarbeiter berichteten 2006 über den Einsatz der iLA bei 90 Patienten mit schwerem ARDS unterschiedlicher Genese. Bereits $2 \mathrm{~h}$ nach Beginn der iLA-Therapie war der arterielle $\mathrm{PCO}_{2}$ im Mittel von 60 auf $36 \mathrm{mmHg}$ abgefallen, während der Oxygenierungsindex im gleichen Zeitraum von 58 auf $82 \mathrm{mmHg}$ anstieg. Die Gesamtüberlebensrate lag mit $41 \%$ über dem Erwartungswert, wobei diese Studie keine Kontrollgruppe untersuchte.

In einer anderen Studie setzten Fischer und Mitarbeiter die iLA als Bridging-Verfahren bis zu einer Lungentransplantation bei Patienten mit chronischen Lungenerkrankungen im Endstadium ein. Insgesamt wurden 12 Patienten behandelt, wiederum mit einem deutlichen Abfall der $\mathrm{CO}_{2}$-Werte, im Mittel von 121 auf $54 \mathrm{mmHg}$, sowie einem moderaten Anstieg der $\mathrm{P}_{\mathrm{a}} \mathrm{O}_{2}$-Werte von 76 auf $89 \mathrm{mmHg}$. Die Behandlungszeiträume lagen zwischen 4 und 32 Tagen. Insgesamt konnten 10 Patienten transplantiert werden, von denen 8 nach 1 Jahr noch am Leben waren. Allerdings hatte auch diese Studie keine Kontrollgruppe.

$\mathrm{Zu}$ den schwerwiegenden Komplikationen der iLA gehören Ischämien der unteren Extremität (auf der Seite der arteriellen Kanüle), Thrombosen der Katheter bzw. der katheterführenden Gefäße und Blutungen. Allerdings lassen sich diese Probleme durch engmaschige klinische Untersuchungen frühzeitig erkennen und zumeist rasch beheben.

\section{Zusammenfassung}

- Tab.15.11 fasst noch einmal die wichtigsten Charakteristika der verschiedenen extrakorporalen Lungenersatzverfahren zusammen. Nach wie vor gibt es keine Daten aus kontrollierten Studien, die einen Überlebensvorteil für extrakorporale Lungenersatzverfahren zeigen können. Somit kann man auch keine Kriterien definieren, nach denen der Einsatz dieser Verfahren generell empfohlen

- Tab. 15.11. Charakteristika der extrakorporalen Lungenersatzverfahren

\begin{tabular}{|l|l|l|}
\hline Verfahren & Pumpe erforderlich & Primäres Therapieziel \\
\hline Venoarteriell (ECMO) & Ja & Ersatz/Support der kardialen Pumpfunktion \\
\hline Venovenös & Ja & Sicherstellung der Oxygenierung \\
\hline Arteriovenös (iLA) & Nein & $\mathrm{CO}_{2}$-Elimination, lungenprotektive Beatmungsstrategie \\
\hline
\end{tabular}

ECMO extrakorporale Membranoxygenierung, iLA »interventional lung assist« 
werden kann. Dies bedeutet zum gegenwärtigen Zeitpunkt mit anderen Worten, dass Indikationen für extrakorporale Verfahren ausschließlich unter individuellen Gesichtspunkten zu stellen sind.

Die klassische venoarterielle ECMO kommt derzeit als Therapie des ARDS kaum zum Einsatz. Quantitativ die größte Bedeutung haben derzeit pumpenlose arteriovenöse Lungenunterstützungsverfahren, die allerdings ebenfalls noch nicht nach den Kriterien der evidenzbasierten Medizin evaluiert wurden. Der Bedarf an komplikationsarmen, gleichzeitig aber effizienten Lungenunterstützungsverfahren besteht jedoch trotz Fortschritten in der konventionellen Beatmung ohne Zweifel fort und es bleibt zu hoffen, dass mit den gegenwärtigen Techniken auch eine Verbesserung der Behandlungsergebnisse erzielt und demonstriert werden kann.

\section{Literatur}

Amato MB, Barbas CS, Medeiros DM et al. (1998) Effect of a protectiveventilation strategy on mortality in the acute respiratory distress syndrome. N Engl J Med 338(6):347-354

Ashbaugh DG, Bigelow DB, Petty TL, Levine BE (1967) Acute respiratory distress in adults. Lancet 2(7511):319-323

Bein T, Weber F, Philipp A et al. (2006) A new pumpless extracorporeal interventional lung assist in critical hypoxemia/hypercapnia. Crit Care Med 34(5):1372-1377

Borges JB, Okamoto VN, Matos GFJ et al. (2006) Reversibility of lung collapse and hypoxemia in early acute respiratory distress syndrome. Am J Respir Crit Care Med 174:268-278

Brochard L, Rauss A, Benito S et al. (1994) Comparison of three methods of gradual withdrawal from ventilatory support during weaning from mechanical ventilation. Am J Respir Crit Care Med 150:896-903

Brower RG, Lanken PN, Maclntyre N et al. (2004) Higher versus lower positive end-expiratory pressures in patients with the acute respiratory distress syndrome. N Engl J Med 351(4):327-336

deJonge E, Schultz MJ, Spanjaard L, Bossuyt PMM, Vroom MB, Dankert J, Kesecioglu J (2003) Effects of selective decontamination of digestive tract on mortality and acquisition of resistant bacteria in intensive care: a randomized controlled trial. Lancet 362:10111016

Ely EW, Baker AM, Dunagan DP et al. (1996) Effect on the duration of mechanical ventilation of identifying patients capable of breathing spontaneously. NEJM 335:1864-1869

Fischer S, Simon AR, Welte T, Hoeper MM, Meyer A, Tessmann R, Gorbandt B, Gottlieb J, Haverich A, Strueber M (2006) Bridge to lung transplantation with the novel pumpless interventional assist NovaLung. J Thorac Cardiovasc Surg 131:719-23

Gattinoni L, Agostoni A, Pesenti A et al. (1980) Treatment of acute respiratory failure with low-frequency positive-pressure ventilation and extracorporeal removal of $\mathrm{CO}_{2}$. Lancet 2(8189):292-294

Gattinoni L, Tognoni G, Pesenti A et al. (2001) Effect of prone positioning on the survival of patients with acute respiratory failure. $\mathrm{N}$ Engl J Med 345:568-573

Gattinoni L, Vagginelli F, Carlesco E, Taccone P, Conte V, Chiumello D, Valenza F, Caironi P, Pesenti A (2003) Decrease in $\mathrm{paCO}_{2}$ with prone position is predictive of improved outcome in acute respiratory distress syndrome. Crit Care Med 31:2727-2733

Griffiths MJD, Evans TW (2005) Inhaled nitric oxide therapy in adults. N Engl J Med 353:2683-2695
Hager DN, Krishnan JA, Hayden DL, Brower RG (2005) Tidal volume reduction in patients with acute lung injury when plateau pressures are not high. Am J Respir Crit Care Med 172:1241-1245

Hill JD, O'Brien TG, Murray JJ et al. (1972) Prolonged extracorporeal oxygenation for acute post-traumatic respiratory failure (shocklung syndrome). Use of the Bramson membrane lung. N Engl J Med 286(12):629-634

Koeman M, van der Ven AJAM, Hak E et al. (2006) Oral decontamination with Chlorhexidin reduces the incidence of Ventilator-associated pneumonia. Am J Respir Crit Care Med 173:1348-1355

Kress, JP, Pohlmann AE, O'Connor MF, Hall JB (2000) Daily interruption of sedative infusions in critically ill patients undergoing mechanical ventilation. N Engl J Med 342:1471-1477

Lellouche F, Mancebo J, Jolliet P et al. (2006) A multicenter randomized trial of computer-driven protocolized weaning from mechanical ventilation. Am J Resp Crit Care Med 174:894-900

MacIntyre NR, Cheng K-CG, McConnell R (1997) Applied PEEP during pressure support reduces the inspiratory threshold load of intrinsic PEEP. Chest 111:188-193

Maclntyre NR, Cook DJ, Guyatt GH (2001) Evidence based guidelines for weaning and discontinuing ventilatory support. American College of Chest Physicians, American Association for Respiratory Care, and American College of Critical Care Medicine. Chest 120 (6 Suppl):375S-484S

Mancebo J, Fernández R, Blanch L et al. (2005) A multicenter trial of prolonged prone ventilation in severe acute respiratory distress syndrome. Am J Respir Crit Care Med 173:1233-1239

Morán I, Zavala E, Fernandez R, Blanch L, Mancebo J (2003) Recruitment manoeuvres in acute lung injury/acute respiratory distress syndrome. Eur Respir J 22:Suppl.42,37s-42s

Morris AH, Wallace CJ, Menlove RL et al. (1994) Randomized clinical trial of pressure-controlled inverse ratio ventilation and extracorporeal $\mathrm{CO}_{2}$ removal for adult respiratory distress syndrome. Am J Respir Crit Care Med 149(2 Pt 1):295-305

Rumbak MJ, Newton M, Truncale T, Schwartz SW, Adams JW, Hazard PB (2004) A prospective, randomized study comparing early percutaneous dilational trecheotomy to prolonged transleryngeal intubation (delayed tracheotomy) in critically ill medical patients. Crit Care Med 32:1689-1694

The Acute Respiratory Distress Syndrome Network (2000) Ventilation with lower tidal volumes as compared with traditional tidal volumes for acute lung injury and the acute respiratory distress syndrome. N Engl J Med 342:1301-1308

The National Heart, Lung and Blood Institute Acute Respiratory Distress Syndrome (ARDS) Clinical trials Network (2004) Higher versus lower positive end expiratoy pressures in patients with the acute respiratory distress syndrome. N Engl J Med 351:327-336

The National Heart, Lung and Blood Institute Acute Respiratory Distress Syndrome (ARDS) Clinical trials Network (2006) Efficiacy and safety of corticosteroids for persistent acute respiratory distress syndrome. N Engl J Med 354:1671-1684

The National Heart, Lung and Blood Institute Acute Respiratory Distress Syndrome (ARDS) Clinical trials Network (2006) Pulmonaryartery versus central venous catheter to guide treatment of acute lung injury. N Engl J Med 354:2213-2224

The National Heart, Lung and Blood Institute Acute Respiratory Distress Syndrome (ARDS) Clinical trials Network (2006) Comparision of Two Fluid-Management Strategies in Acute Lung Injury. N Engl J Med 354:2564-2575

Spragg RG, Lewis JF, Walmrath HD et al. (2004) Effect of recombinant surfactant protein $\mathrm{C}$-based surfactant on the acute respiratory distress syndrome. N Engl J Med 351:884-892

Zapol WM, Snider MT, Hill JD et al. (1979) Extracorporeal membrane oxygenation in severe acute respiratory failure. A randomized prospective study. Jama 242(20):2193-2196 


\subsection{Lungentransplantation}

T. Kramm, H.-J. Schäfers

\subsubsection{Einleitung}

Die Lungentransplantation hat in den letzten 25 Jahren eine sehr positive Entwicklung vollzogen. Erst 1981 wurde die erste erfolgreiche Verpflanzung von Herz und Lungen durchgeführt [86]. Es folgten 1983 die erste einseitige und 1986 die erste doppelseitige isolierte Lungentransplantation [1, 76]. Das Überwinden der initialen Probleme, die Verbesserung der Organkonservierung und auch die Einführung neuer Immunsuppressiva führten zu einer schnellen, weltweiten Verbreitung dieser Therapiekonzepte. In der zweiten Hälfte der 1990er-Jahre wurden weltweit jährlich 1500-2000 Lungentransplantation durchgeführt [107].

Aufgrund der Knappheit an geeigneten und verfügbaren Spenderorganen ist die Zahl der Lungentransplantationen in den letzten Jahren rückläufig [107]. Weiterhin noch nicht gelöst sind klinische Probleme der akuten wie auch der chronischen Transplantatdysfunktion [11, 95, 106]. Dennoch besitzt die Lungentransplantation heute einen akzeptierten und etablierten Platz in der Behandlung des terminalen pulmonalen Versagens, sei es auf dem Boden parenchymatöser oder vaskulärer Erkrankungen.

\subsubsection{Empfängerauswahl und Entscheidungskriterien}

Wie in anderen Formen der Organtransplantation auch, muss die Entscheidung für eine Lungentransplantation für jeden Patienten individuell allgemeine und erkrankungsspezifische Aspekte berücksichtigen (s. Übersicht). Grundvoraussetzungen für die Transplantation sind:
- Die Grunderkrankung ist irreversibel und konservative, d.h. organerhaltende Therapiemöglichkeiten sind ausgeschöpft.

- Es bestehen Erfolgsaussichten nach einer Transplantation.

- Die Spontanprognose des Patienten ist abschätzbar und somit die Lungentransplantation notwendig und dringlich $[2,70]$.

\section{Allgemeine Kriterien der Empfängerselektion \\ - Lungenerkrankung im Endstadium (Lebens- erwartung 12-24 Monate) \\ - klinische Verschlechterung der Lungenfunktion trotz optimaler konservativer Therapie \\ - Komplikationen (Pneumonie, Pneumothorax, Hämoptyse, Synkopen) \\ - ausreichender Ernährungszustand \\ - Motivation des Patienten \\ - stabiles soziales Umfeld}

Die Möglichkeit organerhaltender Behandlungsverfahren hängt im Wesentlichen von der Grunderkrankung der Patienten ab. Bei den pulmonal-vaskulären Erkrankungen konnten erhebliche Fortschritte in den letzten Jahren durch eine medikamentöse Therapie erzielt werden. Mit der parenteralen oder inhalativen Gabe von Prostanoiden [12, 72], der Gabe von Phosphordiesteraseinhibitoren (Sildenafil) [41] oder der Behandlung durch Endothelinrezeptorantagonisten $[46,89]$ wurde die Prognose der primären pulmonalen Hypertonie drastisch verbessert. Bei der pulmonalen Hypertonie ist somit eine durchgeführte Therapie oder zumindest ein erfolgloser Therapieversuch zu fordern, bevor der Erkrankungsprozess als irreversibel eingestuft werden kann. Ist die pulmonale Hypertonie thrombembolischer Genese, so stellt die pulmonale Endarteriektomie ein kausales und effektives Behand-
Einzellungentransplantationen

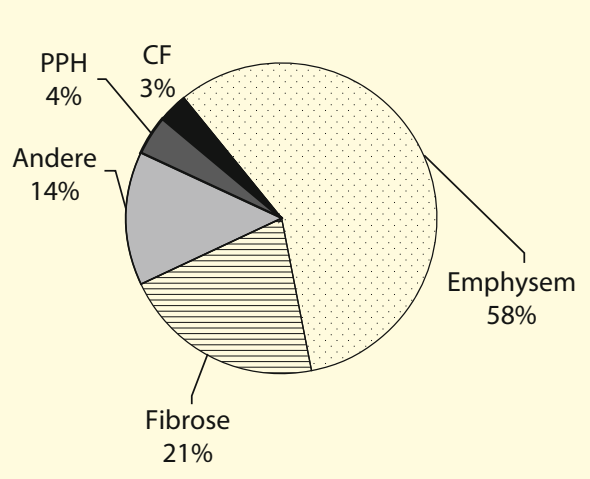

Doppellungentransplantationen

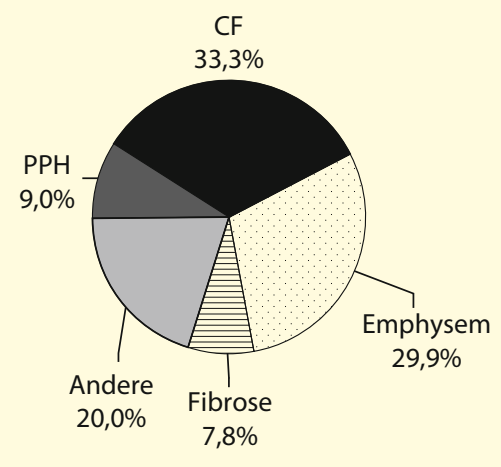

- Abb. 15.23. Indikationen zur Lungentransplantation: Auszug aus dem Register der International Society for Heart and Lung Transplantation (ISHLT) 1983-2005. CF Mukoviszidose, $P P H$ primäre pulmonale Hypertonie 
lungsverfahren dar, das einer Transplantation - wann immer möglich - vorzuziehen ist $[48,55]$.

Bei der Mukoviszidose konnte eine drastische Verbesserung der Spontanprognose durch konsequente Physiotherapie und antibiotische Behandlung erreicht werden. Diese Behandlungsmaßnahmen sollten ausgeschöpft werden, bevor die Transplantation in Betracht gezogen wird. Ähnliches gilt für die immunsuppressive Behandlung der Lungenfibrose [96].

Weniger eindeutig ist die Situation in der konservativen Behandlung des Emphysems. Es ist naheliegend und sollte selbstverständlich sein, dass ein Nikotinkonsum eingestellt sein muss, bevor eine Transplantation infrage kommt. Die muskuläre Rehabilitation bei der chronisch obstruktiven Lungenerkrankung verbessert die Lebensqualität, hat jedoch einen nur geringen Einfluss auf die Prognose der Erkrankung [31]. Die Substitutionsbehandlung beim $\alpha$-1-Antitrypsinmangel hat bislang keine Veränderungen der Spontanprognose bewirken können [93]. Mit der operativen Lungenvolumenreduktion besteht für ausgewählte Patienten mit einem apikal betonten Emphysem die Möglichkeit der funktionellen Verbesserung über mehrere Jahre, offensichtlich kann auch die Prognose der Grunderkrankung positiv beeinflusst werden. Letztendlich bleibt diese Option jedoch nur sehr wenigen Patienten vorbehalten $[25,38,63]$.

Die Berücksichtigung der voraussichtlichen Erfolgschancen nach einer Transplantation ist nicht nur sinnvoll, sondern auch von dem gültigen Transplantationsgesetz gefordert (BÄK 2000). Die Prognose nach Transplantation wird wesentlich bestimmt durch Alter des Patienten, Begleiterkrankungen, aber auch Motivation und Kooperationsbereitschaft.

Die Daten des internationalen Registers der Society for Heart and Lung Transplantation zeigen, dass die Lebenserwartung nach Transplantation bei Kindern unter 10 Jahren und bei älteren Menschen über 60 Jahren gegenüber dem Durchschnitt deutlich vermindert ist [8, 107]. Eine kausale Analyse lassen die Registerzahlen jedoch nicht zu. Es ist unklar, ob in erster Linie das Alter oder nicht viel mehr andere Begleitumstände die verminderte Überlebenswahrscheinlichkeit bedingen. Die gegenwärtigen Empfehlungen der Deutschen Gesellschaft für Pneumologie beinhalten eine obere Altersgrenze von 65 Jahren [70]. Wichtig ist jedoch festzuhalten, dass nicht das Alter allein ausschlaggebend ist, vielmehr müssen das biologische Alter und Begleiterkrankungen in der Zusammenschau gesehen werden.

Begleiterkrankungen spielen eine zentrale Rolle in der Prognoseabschätzung. Eine maligne Grunderkrankung und das Vorkommen anderer maligner Erkrankungen in den letzten Jahren vor Entscheidung zur Transplantation sind mit einem deutlich erhöhten Rezidivrisiko nach Transplantation assoziiert [2, 3, 79]. Ursächlich ist die erforderliche Immunsuppression $\mathrm{zu}$ sehen, die $\mathrm{zu}$ einer Verschiebung der Balance zwischen malignem Wachstum und Immunabwehr führt (s. Übersicht).

Erkrankungen des atheroskleroseassozierten Formenkreises (Karotisstenose, koronare Herzerkrankung, arterielle Verschlusskrankheit) bedingen auch ohne Lungenerkrankung eine gegenüber der Norm verminderte Lebenserwartung. Ein pauschaler Ausschluss solcher Erkrankungen in der Entscheidung zur Transplantation erscheint wahrscheinlich jedoch nicht gerechtfertigt [57, 59, 94, 99].

Schwere und irreversible Funktionseinschränkungen anderer Organe (z. B. Niere, Leber) sind dagegen kritischer zu sehen. Prinzipiell ist zwar die kombinierte Transplantation von Lunge und Niere, bzw. Lunge und Leber möglich, bislang liegen jedoch nur limitierte Erfahrungen vor [2]. Es ist davon auszugehen, dass solche kombinierten Organtransplantationen im Vergleich zur isolierten Lungentransplantation mit einer verminderten Langzeitprognose verbunden sind.

Vorausgegangene Operationen erhöhen die technische Komplexität der Transplantation, haben jedoch keinen Einfluss auf die mittel- und langfristige Prognose nach Transplantation [32, 36]. Sie stellen somit keine akzeptable Kontraindikation gegen eine Transplantation dar. Gleichfalls ist die Beatmung vor Transplantation kein negativer Prognoseindikator und somit keine Kontraindikation gegen die Lungentransplantation [14, 16, 43, 67].

Im Einzelfall schwierig zu bewerten ist die Motivation und Kooperationsbereitschaft bzw. -fähigkeit des Patienten. Bei der Organtransplantation allgemein, bei der Lungentransplantation aber noch wesentlich mehr als bei der Verpflanzung anderer Organe, ist die Kooperation des Patienten gefordert, um Komplikationen frühzeitig und genau erkennen und behandeln zu können. Im Regelfall setzt dies Einsicht in die Erkrankung und die erforderlichen Maßnahmen voraus [70].

Kontraindikationen für eine Lungentransplantation

- Aktueller Infekt oder instabiler klinischer Status

- nicht ausbehandeltes Malignom

- signifikante Funktionseinschränkung anderer vitaler Organe (Herz, Niere, ZNS)

- schwere koronare Herzerkrankung mit eingeschränkter Herzfunktion

- aktiver Nikotinkonsum

- Drogen oder Alkoholabhängigkeit

- psychische Erkrankungen unter medikamentöser Behandlung

- HIV-, Hepatitis-B-, Hepatitis-C-Infektion (Leberbeteiligung) 
Die prognostische Einschätzung der Grunderkrankung spielt eine zentrale Rolle und muss die Dynamik der jeweiligen Erkrankung berücksichtigen (s. Übersicht). Für die Mukoviszidose hat sich das Ausmaß der $\mathrm{CO}_{2}$-Retention als prognostisch wichtiger herausgestellt, als die Einschränkung der Lungenfunktionsparameter (FEV1, VK) [52]. Bei Auftreten eines Spontanpneumothorax oder relevanter Hämoptysen, die durch eine Infektbehandlung oder Embolisation nicht ausreichend stabilisiert werden kann, wird die Grunderkrankung zusätzlich prognostisch beeinflusst. In solchen Situationen kann die Entscheidung zur Transplantation trotz eines bis dahin noch relativ stabilen Krankheitsverlaufs notwendig sein $[34,111]$.

Bei der pulmonalen Hypertonie sind die Symptome einer Herzinsuffizienz (NYHA-Stadium II-III) und die invasiv bestimmten Indikatoren für ein vermindertes Herzzeitvolumen (zentraler Venendruck, Herzindex, gemischt-venöse Sauerstoffsättigung) zurzeit die zuverlässigsten Prognosekriterien [27]. Schwierig ist die prognostische Abschätzung und therapeutische Entscheidung beim heute seltenen Eisenmenger-Syndrom. Die traditionell gefürchteten Hämoptysen und ventrikulären Arrhythmien sind relativ selten und das Ausmaß der begleitenden Zyanose ist kein Prognoseindikator. Am ehesten scheint das Auftreten von Symptomen einer Herzinsuffizienz entsprechend dem Stadium III der New York Heart Association (NYHA-Stadium III) die eingeschränkte Überlebenswahrscheinlichkeit vorherzusagen [2].

Die prognostische Beurteilung der obstruktiven Lungenerkrankungen ist die schwierigste. Prinzipiell ist bei Verminderung der FEV1 unter 20\% des Sollwerts die 5-Jahres-Überlebenswahrscheinlichkeit eingeschränkt $[10,96]$. Kritische Analysen der Überlebenswahrscheinlichkeit auf der Warteliste und nach Transplantation haben jedoch gezeigt, dass konventionelle Prognoseparameter keine ausreichende Diskriminierung erlauben [6]. Klinische Parameter (Zahl der Infekte, körperliche Leistungsfähigkeit mit 6-Minuten-Gehstrecke trotz optimaler Rehabilitation, Notwendigkeit der nichtinvasiven Beatmung) und auch das Ausmaß der respiratorischen Globalinsuffizienz sind wahrscheinlich bessere Indikatoren für das Vorliegen eines Terminalstadiums der Erkrankung [21].

Die Lungenfibrose stellt eine seltenere Indikation zur Transplantation dar. In Abhängigkeit vom Ausmaß der Parenchymdestruktion besteht zusätzlich eine moderate oder schwere pulmonale Hypertonie [106]. Diese Erkrankung weist eine variable Progredienz mit entsprechend hoher Letalität noch vor Transplantation auf [44, 92]. Eine progrediente Hyperkapnie ist ein Alarmsignal, das auch bei klinisch stabilen oder wenig symptomatischen Patienten auf eine Progredienz der Erkrankung hinweist (s. Übersicht) [2].
Funktionskriterien zur Transplantationsindikation nach Grunderkrankung

COPD, a-1-Antitrypsinmangelemphysem:

- FEV1<25\% unter Bronchodilatatorentherapie

- Hypoxie in Ruhe: $\mathrm{P}_{\mathrm{a}} \mathrm{O}_{2}<55-60 \mathrm{mmHg}$

- Hyperkapnie

- sekundäre pulmonale Hypertonie

- Klinik: rasche Abnahme der FEV1 oder lebensbedrohliche Infektexazerbationen

Mukoviszidose:

- FEV $1<30 \%$ unter Bronchodilatatorentherapie

- Hypoxie in Ruhe: $\mathrm{P}_{\mathrm{a}} \mathrm{O}_{2}<55 \mathrm{mmHg}$

- Hyperkapnie

- Klinik: zunehmende Heftigkeit und Häufigkeit der pulmonalen Infekte

idiopathische pulmonale Fibrose:

- VC, TLC $<60-65 \%$

- Hypoxie in Ruhe

- sekundäre pulmonale Hypertonie

- klinische, radiologische oder physiologische Progression unter maximaler Therapie

primäre pulmonalarterielle Hypertonie:

- NYHA-Klasse III oder IV

- mittlerer rechtsatrialer Druck $>10 \mathrm{mmHg}$

- mittlerer pulmonalarterieller Druck $>50 \mathrm{mmHg}$

- Herzindex $<2,5 \mathrm{l} / \mathrm{min} / \mathrm{m}^{2}$

\subsubsection{Organspenderkriterien}

Prinzipiell ist jeder hirntote Organspender mit erhaltener Lungenfunktion zur Lungenspende geeignet (s. Übersicht). Die Tatsache, dass das Herz und beide Lungen eines Organspenders separat entnommen und transplantiert werden können, hat zu einer erheblichen Erweiterung des Organpools geführt. Die Zahl der verfügbaren Organe ist jedoch dadurch beschränkt, dass der primäre Krankheitsmechanismus, der zum Hirntod führt, auch die Lunge schädigen kann (z. B. Thoraxtrauma mit Kontusionen, Aspiration, pulmonaler Schaden nach Massentransfusion). Unter der Beatmung des hirntoten Patienten kann vor allem eine Pneumonie auftreten, die eine erfolgreiche Lungentransplantation infrage stellt. Der Hirntod mit der begleitenden Entgleisung der Kreislaufregulation und einer konsekutiven Volumensubstitution kann ein ausgeprägtes Lungenödem nach sich ziehen. 
Allgemeine Spenderkriterien

- Alter unter 55 Jahre

- keine bekannte Lungenerkrankung

- unauffälliges Röntgenbild

- $\mathrm{PO}_{2}>300 \mathrm{mmHg}$ bei $100 \%$ Sauerstoff, PEEP $5 \mathrm{cmH}_{2} \mathrm{O}$

- unauffällige Bronchoskopie

- kein akuter pulmonaler Infekt

- Hepatitis-B-, Hepatitis-C- und HIV-negativ

- passende Blutgruppe

- Organgröße (Spendergröße und -gewicht, TLC)

Aufgrund der in der Übersicht genannten Einschränkungen können zurzeit nur 20-30\% der Lungen von Multiorganspendern zur Lungentransplantation genutzt werden. In dem Bemühen, die Zahl der verfügbaren Organe zu erhöhen, sind die ursprünglich strikten Kriterien für Oxygenierung und Spenderalter inzwischen aufgeweicht worden [17]. Die bisherigen Erfahrungen zeigen, dass eine eingeschränkte Oxygenierung als alleiniges Kriterium kein guter Prädiktor für eine eingeschränkte Transplantatfunktion nach Transplantation ist [4]. Die Ausweitung der Altersgrenzen für die Lungenspende hat bislang nicht zu einer gravierenden Verschlechterung der Transplantationsergebnisse geführt, wenn auch die internationalen Registerzahlen prinzipiell eine Einschränkung der Überlebenswahrscheinlichkeit suggerieren [26]. Zurzeit wird diskutiert, ob eine Zuordnung von Lungen von älteren Spendern für ältere Empfänger (»old for old «) ein akzeptabler Kompromiss ist [73].

\subsubsection{Organkonservierung}

Die Konservierung der Lungentransplantate ist inzwischen weltweit weitgehend standardisiert. In den meisten Zentren hat sich die Verwendung einer extrazellulären Lösung (Perfadex ${ }^{\circledast}$ ) durchgesetzt, die antegrad in die Pulmonalarterien infundiert wird [53]. Die zusätzliche Gabe von Prostanoiden inhalativ oder parenteral hat einen weiteren positiven Effekt [82]. Mit diesen Konservierungsmöglichkeiten sind heute Ischämiezeiten von 6-8 h problemlos zu überbrücken. Das Auftreten einer initialen Transplantatdysfunktion korreliert in diesem Zeitraum nicht mit der tatsächlichen Dauer der Ischämie.

\subsubsection{Transplantationstechnik}

Die Operationstechnik ist ebenfalls weitestgehend standardisiert. Für die einseitige Lungentransplantation wird der betreffende Hemithorax durch eine antero- oder posterolaterale Thorakotomie eröffnet. Die native Lunge wird entfernt, Bronchus, Pulmonalarterie und linksatriale Manschette werden an die korrespondierenden Empfängerstrukturen anastomosiert. Zur Minimierung bronchialer Komplikationen hat sich die ausgeprägte Kürzung des Spenderbronchus bewährt [29]. In den letzten Jahren sind kürzere Inzisionen unter dem Schlagwort der »minimalen Invasivität« propagiert worden. Bislang steht der Nachweis eines Vorteils dieser limitierten Schnitte aus. Nachteil dieser begrenzten Zugänge ist sicherlich der eingeschränkte operative Spielraum, der bei Vorliegen von Verwachsungen oder ausgeprägten Kollateralgefäßen die Operation verlängert und an Exaktheit einbüßen lässt.

Für die bilaterale Transplantation wird meist eine bilaterale, transsternale Thorakotomie im 4. Interkostalraum verwendet, alternativ sind auch 2 getrennte anterolaterale Thorakotomien möglich. Die Technik der Entfernung der nativen Lunge und der Implantation des Transplantats ist identisch mit der Technik der Einzellungentransplantation [75].

Komplexer ist die chirurgische Technik der kombinierten Transplantation von Herz und Lungen. Diese Operation wird unter Einsatz der Herz-Lungen-Maschine durchgeführt. Der operative Zugangsweg ist eine mediane Sternotomie. Nach Entfernung des Herzens auf der Ebene des rechten Vorhofs werden die Hilusstrukturen mobilisiert und die linke und rechte Lunge explantiert. Hierbei müssen auf beiden Seiten die Nn. phrenici und links zusätzlich der N. laryngeus recurrens am Ligamentum Botalli erhalten werden. Die Implantation der Spenderorgane erfolgt en-bloc durch Anastomosierung von Trachea, rechtem Vorhof und abschließend der Aorta $[45,54]$.

Für infizierte Lungenerkrankungen (Mukoviszidose) ist die bilaterale Transplantation erforderlich, um keinen infektiösen Fokus nach der Transplantation zu hinterlassen [7, 98]. Für die pulmonale Hypertonie hat sich die bilaterale Transplantation bewährt, da hier im postoperativen Verlauf weniger Probleme durch Missverhältnis von Ventilation und Perfusion zu erwarten sind. Das Eisenmenger-Syndrom kann bei Vorliegen einfach korrigierbarer kardialer Vitien durch kardiale Korrektur und bilaterale Transplantation behandelt werden, ansonsten ist es die letzte Domäne der kombinierten Herzlungentransplantation [9, 39, 108].

Die Frage der uni- bzw. bilateralen Transplantation bei obstruktiven Lungenerkrankungen ist Gegenstand kontroverser Diskussionen. Die einseitige Transplantation bietet den Vorteil des geringeren Operationstraumas und der günstigeren Organökonomie. Nach der Transplantation ist die funktionelle Reserve jedoch eingeschränkt und gelegentlich wird ein Perfusions-Ventilations-Mismatch mit Überblähung der nativen Lunge und eine Kompression der transplantierten Lunge beobachtet. Die bilaterale Transplantation bringt das größere perioperative Trauma mit sich, erlaubt jedoch die schnellere Rehabilitation und führt zu besserem Langzeitüberleben $[9,15,81]$. 
Bei der Lungenfibrose ist eine unilaterale Transplantation funktionell adäquat [104]. Zurzeit ist noch unklar, ob die Fibrose eine prämaligne Erkrankung darstellt, bei der nach einseitiger Transplantation ein erhöhtes Risiko einer malignen Entartung der verbliebenen, nativen Lunge zu erwarten ist [28, 109]. Die gegenwärtige Knappheit an Transplantatlungen lässt einen generellen Einsatz der bilateralen Transplantation bei obstruktiven und fibrosierenden Lungenerkrankungen als nicht gerechtfertigt erscheinen.

Bei der unilateralen Transplantation richtet sich die Wahl der zur transplantierenden Seite in der Regel nach der relativen Destruktion der nativen Lunge. In der Regel wird die stärker befallene ersetzt. Im Zweifelsfall bietet sich bei der Lungenfibrose die linksseitige Transplantation an, da sich hier ein überdimensioniertes Transplantat besser entfalten kann. Bei der obstruktiven Lungenerkrankung wird meist die rechte Seite bevorzugt, da so die Wahrscheinlichkeit einer Kompression des Transplantats und einer mediastinalen Verlagerung geringer sind.

\subsubsection{Postoperatives Management}

Die frühe postoperative Betreuung muss die typischen Komplikationen berücksichtigen und möglichst vermeiden. Prinzipiell impliziert der Hirntod mit allen Begleitaspekten der Organspende ein erhöhtes Risiko einer bakteriellen Besiedlung oder einer beginnenden Pneumonie. Jede mikrobiologische Diagnostik ist jedoch automatisch von einer Laten $z$ von mindestens $48 \mathrm{~h}$ begleitet, die erforderlich sind, um objektive Informationen über eine Keimbesiedlung und ein Antibiogramm zu erbringen. Möglicherweise begünstigen Konservierung und Ischämiereperfusionsschäden die Entwicklung einer Pneumonie aus einer ansonsten als wenig relevant eingestuften Keimbesiedelungen.

Prinzipiell hat es sich bewährt, jede frisch transplantierte Lunge als potenziell pneumonisch verändert $\mathrm{zu}$ betrachten und eine empirische Therapie zu beginnen. Diese Therapie kann dann nach Erhalt der mikrobiologischen Daten abgesetzt oder modifiziert werden. Das zu erwartende Keimspektrum entspricht dem einer nosokomialen Infektion. Die Kombination aus einem Breitspektrumpenizillin, Aminoglykosid und einem Antibiotikum gegen einen gram-positiven Erreger berücksichtigt die überwiegende Mehrzahl möglicher Keime. Liegt eine infizierte Grunderkrankung beim Empfänger vor (z. B. Mukoviszidose), sollte die Resistenzlage der vorliegenden chronischen Empfängerbesiedelung mit berücksichtigt werden $[56,90]$.

Die Beatmung nach Lungentransplantation entspricht in ihren Grundprinzipien der postoperativen Intensivmedizin. Wichtig ist das Vermeiden eines Barotrauma. Dieses muss besonders für die Konstellation der unilateralen Transplantation bei Fibrose berücksichtigt werden. In dieser Konstellation wird aufgrund der unterschiedlichen Compliance-Verhältnisse meist mehr als 70\% des Zugvolumens in das Transplantat gehen. Es empfiehlt sich somit die Einstellung eines Atemzugvolumens ähnlich wie nach einer Pneumonektomie.

Die Immunsuppression wird traditionell mit einer intraoperativ verabreichten Bolusgabe von Methylprednisolon begonnen (10-15 mg/kg). Postoperativ wird zusätzlich mit der Applikation eines Kalzineurininhibitors sowie eines Antimetaboliten begonnen. Trotz Entwicklung neuer Immunsuppressiva wird Cyclosporin weiterhin bei 40-50\% der Lungentransplantation eingesetzt [107].

Tacrolimus ist eine neuere und von manchen bevorzugte Alternative. Es scheint gegenüber Cyclosporin den Vorteil von weniger Abstoßungsreaktionen im postoperativen Verlauf zu haben [80, 85, 105]. Aufgrund der Tatsache, dass keine gute interindividuelle Korrelation zwischen Cyclosporin- bzw. Tacrolimusblutspiegeln und der spezifischen immunsuppressiven Wirkung besteht, ist die Interpretation der bisherigen Daten schwierig [13].

Azathioprin ist auch weiterhin Teil der Routineimmunsuppression bei $40 \%$ der Transplantationen. Neuere Immunsuppressiva wie Mycofenolat-Mofetil oder Sirolimus und Everolimus werden zunehmend eingesetzt [40, $69,100,107]$. Ob diese eindeutige Vorteile gegenüber Azathioprin haben, ist bislang nicht gut geklärt [42, 80].

Die Gabe von spezifischen Antikörpern als Teil der initialen Immunsuppression wird nicht einheitlich gehandhabt [74]. Beim überwiegenden Teil der Lungentransplantationen werden keinerlei Antikörper eingesetzt; bei ca. 30\% kommen ATG oder Interleukin-2-Antikörper zum Einsatz [50].

Flüssigkeitsbilanz und Kreislaufmanagement entsprechen den gängigen intensivmedizinischen Prinzipien. Aufgrund der Neigung zur Ausbildung eines interstitiellen und alveolären Ödems hat es sich bewährt, einen pulmonalarteriellen Mitteldruck von weniger als $25 \mathrm{mmHg}$ anzustreben.

Nach Entwöhnung von der Beatmung konzentriert sich die weitere Nachsorge auf fortgesetzte muskuläre Rehabilitation und engmaschige und subtile klinische Überwachung bezüglich des Auftretens von Infekten bzw. einer Abstoßungsreaktion.

\subsubsection{Spezifische Komplikationen}

\section{Infektion}

Wie nach allen Organtransplantationen ist das Risiko von Infekten erhöht und die Lunge ist das am häufigste befallene Organ. Bakterielle Infekte sind besonders häufig innerhalb der ersten 2-3 postoperativen Wochen, prinzipiell sind alle Keime des Erregerspektrums mögliche Pathogene [33]. Mit viralen Infektionen ist $\mathrm{zu}$ diesem 
Zeitpunkt jenseits der 3. postoperativen Woche zu rechnen. Infekte mit atypischen Erregern sind überwiegend Probleme im langfristigen Verlauf. Die gefürchteten Infektion durch Pneumocystis carinii lassen sich durch die prophylaktische Gabe von Trimethoprim/Cotrimoxazol (875/125 mg alle 2-3 Tage) weitestgehend verhindern.

Unter den Pathogenen ist das Cytomegalie-(CMV-) Virus das quantitativ wichtigste, das durch Reaktivierung sowie Neuinfektion schwere Krankheitsverläufe induzieren kann. Klinische Beobachtungen legen nahe, dass auch durch virale Infekte eine chronische Transplantatdysfunktion (obliterative Bronchiolitis, s. dort) induziert werden kann [51, 97]. Für die Therapie von CMV-Infektionen stehen Ganciclovir und Valganciclovir zur Verfügung [35]. Umstritten ist der Wert einer direkt postoperativen Prophylaxe in Risikokonstellationen (z. B. CMV-negativer Empfänger, CMVpositives Organ). Die postoperative prophylaktische Gabe von Ganciclovir scheint im Wesentlichen den Zeitpunkt eines CMV-Infekts zu verschieben [101, 102].

\section{Abstoßungsreaktionen}

Die Diagnose der akuten Abstoßungsreaktionen ist weiterhin im Wesentlichen eine klinische. Radiologisch nachweisbares Infiltrat, eingeschränkte Oxygenierung, ein Anstieg der Leukozyten oder vermehrte Atemarbeit legen die Diagnose nahe. Der histologische Nachweis in einer transbronchialen Biopsie hilft bei der Diagnosestellung, ist jedoch in Anbetracht der heterogenen Ausprägung der abstoßungsbedingten Veränderung im Lungenparenchym nicht sensitiv. Die bleibende Besserung auf die höher dosierte Immunsuppression ist weiterhin der zuverlässigste, wenn auch nur retrospektive Beweis einer Abstoßungsreaktion. Hiervon $\mathrm{zu}$ trennen ist die chronische Form der Abstoßung, die als chronische Transplantatdysfunktion oder obliterative Bronchiolitis bezeichnet wird.

\section{Bronchiale Komplikationen}

Diese spezifische Komplikation der Lungentransplantation war in der Frühphase eine der größten Hürden. Die Ursache für eine Dehiszenz der bronchialen Anastomose oder nekrotisierende und stenosierende Veränderungen der distalen Atemwege ist am ehesten in einer Ischämie der transplantierten Bronchien zu sehen. Mit der Transplantation wird die nutritive Blutversorgung der Bronchialarterien unterbrochen und die transplantierten Atemwege werden noch über bronchopulmonale Kollateralen aus dem pulmonalarteriellen Blutfluss ernährt. Durch pharmakologische Verbesserung der Mikrozirkulation und Aufrechterhalten eines adäquaten pulmonalarteriellen Drucks kann eine Verbesserung dieser Durchblutung erzielt werden. Von entscheidender Bedeutung ist die Kürze der transplantierten Atemwege.
Wichtig ist es zusätzlich, Ursachen für eine nekrotisierende Entzündung zu eliminieren. Hierzu hat sich die aggressive und unter anderem auch inhalative Behandlung von Bakterien und Pilzen, insbesondere Staphylokokken und Aspergillen, bewährt.

Unter Einsatz aller Maßnahmen sind diese Komplikationen heute selten geworden und werden mit einer Inzidenz von weniger als $2 \%$ beobachtet. Stenosen der transplantierten Atemwege können vorübergehend durch eine Dilatation behandelt werden. Der Einsatz endobronchialer Stents ist nur in wenigen Fällen langfristig erfolgreich gewesen [22, 91]. Die Resektion stenosierter Atemwege oder eine Retransplantation verbleiben als die langfristig besten Behandlungsoptionen.

\section{Malignome}

Nach jeder Organtransplantation stellt die unumgängliche Immunsuppression einen tiefen Eingriff in die immunologische Kompetenz des Organismus dar. Dementsprechend kommt es als Langzeitkomplikation zur Entwicklung bösartiger Tumore [3]. Sie betreffen vor allem Haut, lymphatische Gewebe, den Gastrointestinaltrakt, aber auch Lunge und Urogenitaltrakt [79, 107]. Abhängig vom Zeitpunkt nach der Transplantation beträgt die Inzidenz von Malignomen 13\% nach 5 Jahren und bis zu 20\% nach 7 Jahren.

Zusätzliche Risikofaktoren stellen Alter und vormaliger Nikotinkonsum dar [5]. Die Letalität einer solchen Diagnose kann unter der nach Transplantation nur limitierten spezifischen Therapie bis $\mathrm{zu} 25 \%$ betragen [19, 78, 84]. Dementsprechend ist die Entwicklung eines Malignoms nach der Bronchiolitis obliterans und septisch verlaufenden Infektionen die dritthäufigste Todesursache im Langzeitverlauf nach Transplantation [107].

\section{Funktionelle Resultate}

Mit der Transplantation ist eine akzeptable oder normale Lungenfunktion erreichbar [60]. Die unilaterale Transplantation führt spirometrisch zu Funktionsdaten, die etwa $60 \%$ des Solls entsprechen. Nach bilateraler Transplantation ist eine annähernde Normalisierung der Spirometrie zu erreichen [62]. Der funktionelle Unterschied zwischen uni- und bilateraler Transplantation hat wenige Konsequenzen für die Leistungsfähigkeit der Patienten im normalen Leben [65]. Treten Komplikationen auf, beinhaltet die bilaterale Transplantation größere physiologische Reserven als die unilaterale [58].

Der pulmonale Gefäßwiderstand ist in beiden Fällen normalisiert, nicht zuletzt durch die hohe Reservekapazität des Lungengefäßbetts. Während die bilaterale Transplantation zu einer Normalisierung des Perfusions-Ventilations-Verhältnisses führt, hängt dieses nach unilateraler Transplantation von der Grunderkrankung ab [39, 66]. 
Sowohl bei obstruktiver Lungenerkrankung als auch pulmonaler Hypertonie kann ein erhebliches Missverhältnis in der Verteilung von Ventilation und Perfusion auftreten $[39,64,110,112]$.

\section{Letalität und Langzeitprognose}

Die perioperative Letalität beträgt je nach Grunderkrankung und abhängig von Einzel- oder Doppellungentransplantation $8-21 \%$. Die häufigsten Todesursachen sind septisch verlaufende Infektionen, CMV-Infektionen, Transplantatversagen, Herzversagen und Abstoßungsreaktionen. Die Mehrzahl der Patienten ist allerdings 6-8 Wochen nach der Transplantation, unterstützt von einer Anschlussheilbehandlung, wieder nahezu normal belastungsfähig und nicht mehr sauerstoffabhängig [24, 30, 103].

Die Hauptproblematik bezüglich des Langzeitüberlebens stellt neben Infekten und rezidivierenden akuten Abstoßungsphasen die chronische Abstoßungsreaktion dar, die zur Entwicklung einer Bronchiolitis obliterans führt (•Tab. 15.12) [23, 37]. Sie beeinflusst die Langzeitergebnisse nach Transplantation hinsichtlich Lebensqualität und Letalität relevant [18, 107]. Richtungweisend für die Klinik ist eine Abnahme der FEV1 und progrediente Luftnot [77]. Wichtig ist eine Bronchoskopie zum Ausschluss einer Atemwegstenose als Differenzialdiagnose [88]. Ergänzt wird die Diagnostik durch eine Computertomographie als Bildgebung $[47,68]$. Eine Biopsie ist nicht erforderlich [20].

Die Ursache für die Entwicklung einer chronischen Abstoßungsreaktion ist unklar. Ein Aspekt ist sicherlich die limitierte immunologische Abklärung vor der Transplantation, denn im Allgemeinen wird als Spenderkriterium neben der TLC nur die AB0-Kompatibilität berücksichtigt $[49,83]$. Inwieweit eine genauere immunologische Differenzierung Erfolg verspricht, ist aus der aktuellen Daten- und Studienlage nicht abzuleiten. Inwieweit Organkonservierung, Häufigkeit von Infekten und akuten Abstoßungsreaktionen Einfluss haben, ist ebenfalls nicht in allen Punkten eindeutig [9, 23, 51, 97]. Eine mögliche Therapieoption besteht im Wechsel der Immunsuppression $[61,87]$. Bei weiterer Verschlechterung muss die Indikation einer Retransplantation überdacht werden [71, 82].

- Tab. 15.12. Bronchiolitis-obliterans-Syndrom (BOS): Scoringsystem

\begin{tabular}{l|l|l}
\hline Score & BOS & FEV1 \\
\hline 0 & Keine & $>80 \%$ des Ausgangswerts \\
\hline 1 & Mild & $66-80 \%$ des Ausgangswerts \\
\hline 2 & Moderat & $51-65 \%$ des Ausgangswerts \\
\hline 3 & Schwer & $<50 \%$ des Ausgangswerts \\
\hline
\end{tabular}

\section{Literatur}

[1] Toronto Lung Transplant Group (1986) Unilateral lung transplantation for pulmonary fibrosis. N Engl J Med 314:1140-5

[2] The American Society for Transplant Physicians (ASTP)/American Thoracic Society (ATS)/European Respiratory Society (ERS)/International Society for Heart and Lung Transplantation (ISHLT) (1998) International guidelines for the selection of lung transplant candidates. Am J Respir Crit Care Med 158:335-9

[3] Adami J, Gabel H, Lindelof B, Ekstrom K, Rydh B, Glimelius B, Ekbom A, Adami HO, Granath F (2003) Cancer risk following organ transplantation: a nationwide cohort study in Sweden. Br J Cancer 89:1221-7

[4] Aigner C, Winkler G, Jaksch P, Seebacher G, Lang G, Taghavi S, Wisser W, Klepetko W (2005) Extended donor criteria for lung transplantation - a clinical reality. Eur J Cardiothorac Surg 27:757-61

[5] Amital A, Shitrit D, Raviv Y, Bendayan D, Sahar G, Bakal I, Kramer MR (2006) Development of malignancy following lung transplantation. Transplantation 81:547-51

[6] Anthonisen NR (1989) Prognosis in chronic obstructive pulmonary disease: results from multicenter clinical trials. Am Rev Respir Dis 140:S95-9

[7] Aris RM, Gilligan PH, Neuringer IP, Gott KK, Rea J, Yankaskas JR (1997) The effects of panresistant bacteria in cystic fibrosis patients on lung transplant outcome. Am J Respir Crit Care Med 155:1699-704

[8] Armitage JM, Kurland G, Michaels M, Cipriani LA, Griffith BP, Fricker FJ (1995) Critical issues in pediatric lung transplantation. J Thorac Cardiovasc Surg 109:60-4; discussion 64-5

[9] Bando K, Armitage JM, Paradis IL et al. (1994) Indications for and results of single, bilateral, and heart-lung transplantation for pulmonary hypertension. J Thorac Cardiovasc Surg 108:1056-65

[10] Bando K, Paradis IL, Keenan RJ et al. (1995) Comparison of outcomes after single and bilateral lung transplantation for obstructive lung disease. J Heart Lung Transplant 14:692-8

[11] Bando K, Paradis IL, Similo S et al. (1995) Obliterative bronchiolitis after lung and heart-lung transplantation. An analysis of risk factors and management. J Thorac Cardiovasc Surg 110:4-13; discussion 13-4

[12] Barst RJ, Rubin LJ, McGoon MD, Caldwell EJ, Long WA, Levy PS (1994) Survival in primary pulmonary hypertension with long-term continuous intravenous prostacyclin. Ann Intern Med 121:409-15

[13] Barten MJ, Rahmel A, Garbade J, Bold A, Bittner HB, Dhein S, Mohr FW, Gummert JF (2005) Pharmacodynamic monitoring of the conversion of cyclosporine to tacrolimus in heart and lung transplant recipients. Transplant Proc 37:4532-4

[14] Bartz RR, Love RB, Leverson GE, Will LR, Welter DL, Meyer KC (2003) Pre-transplant mechanical ventilation and outcome in patients with cystic fibrosis. J Heart Lung Transplant 22:433-8

[15] Bavaria JE, Pochettino A, Kotloff RM, Rosengard BR, Wahl PM, Roberts JR, Palevsky HI, Kaiser LR (1998) Effect of volume reduction on lung transplant timing and selection for chronic obstructive pulmonary disease. J Thorac Cardiovasc Surg 115:9-17; discussion 17-8

[16] Baz MA, Palmer SM, Staples ED, Greer DG, Tapson VF, Davis DD (2001) Lung transplantation after long-term mechanical ventilation: results and 1-year follow-up. Chest 119:224-7

[17] Bhorade SM, Vigneswaran W, McCabe MA, Garrity ER (2000) Liberalization of donor criteria may expand the donor pool without adverse consequence in lung transplantation. J Heart Lung Transplant 19:1199-204

[18] Burke CM, Theodore J, Dawkins KD et al. (1984) Post-transplant obliterative bronchiolitis and other late lung sequelae in human heart-lung transplantation. Chest 86:824-9

[19] Caforio AL, Fortina AB, Piaserico S et al. (2000) Skin cancer in heart transplant recipients: risk factor analysis and relevance of immunosuppressive therapy. Circulation 102:III222-7

[20] Chacon RA, Corris PA, Dark JH, Gibson GJ (2000) Tests of airway function in detecting and monitoring treatment of obliterative 
bronchiolitis after lung transplantation. J Heart Lung Transplant 19:263-9

[21] Connors AF Jr, Dawson NV, Thomas C et al. (1996) Outcomes following acute exacerbation of severe chronic obstructive lung disease. The SUPPORT investigators (Study to Understand Prognoses and Preferences for Outcomes and Risks of Treatments). Am J Respir Crit Care Med 154:959-67

[22] Cooper JD, Pearson FG, Patterson GA, Todd TR, Ginsberg RJ, Goldberg $M$, Waters $P$ (1989) Use of silicone stents in the management of airway problems. Ann Thorac Surg 47:371-8

[23] Cooper JD, Billingham M, Egan T et al. (1993) A working formulation for the standardization of nomenclature and for clinical staging of chronic dysfunction in lung allografts. International Society for Heart and Lung Transplantation. J Heart Lung Transplant 12:713-6

[24] Cooper JD, Patterson GA, Trulock EP (1994) Results of single and bilateral lung transplantation in 131 consecutive recipients. Washington University Lung Transplant Group. J Thorac Cardiovasc Surg 107:460-70; discussion 470-1

[25] Cooper JD, Patterson GA, Sundaresan RS, Trulock EP, Yusen RD, Pohl MS, Lefrak SS (1996) Results of 150 consecutive bilateral lung volume reduction procedures in patients with severe emphysema. J Thorac Cardiovasc Surg 112:1319-29; discussion 1329-30

[26] Dahlman S, Jeppsson A, Schersten H, Nilsson F (2006) Expanding the donor pool: lung transplantation with donors 55 years and older. Transplant Proc 38:2691-3

[27] D'Alonzo GE, Barst RJ, Ayres SM et al. (1991) Survival in patients with primary pulmonary hypertension. Results from a national prospective registry. Ann Intern Med 115:343-9

[28] Davies HR, Richeldi L, Walters EH (2003) Immunomodulatory agents for idiopathic pulmonary fibrosis. Cochrane Database Syst Rev: CD003134

[29] De Hoyos A, Maurer JR (1992) Complications following lung transplantation. Semin Thorac Cardiovasc Surg 4:132-46

[30] de Hoyos AL, Patterson GA, Maurer JR, Ramirez JC, Miller JD, Winton TL (1992) Pulmonary transplantation. Early and late results. The Toronto Lung Transplant Group. J Thorac Cardiovasc Surg 103:295-306

[31] De Meester J, Smits JM, Persijn GG, Haverich A (1999) Lung transplant waiting list: differential outcome of type of end-stage lung disease, one year after registration. J Heart Lung Transplant 18:563-71

[32] Detterbeck FC, Egan TM, Mill MR (1995) Lung transplantation after previous thoracic surgical procedures. Ann Thorac Surg 60:139-43

[33] Dobbin C, Maley M, Harkness J, Benn R, Malouf M, Glanville A, Bye $P$ (2004) The impact of pan-resistant bacterial pathogens on survival after lung transplantation in cystic fibrosis: results from a single large referral centre. J Hosp Infect 56:277-82

[34] Doershuk CF, Stern RC (1999) Timing of referral for lung transplantation for cystic fibrosis: overemphasis on FEV1 may adversely affect overall survival. Chest 115:782-7

[35] Duncan SR, Grgurich WF, lacono AT et al. (1994) A comparison of ganciclovir and acyclovir to prevent cytomegalovirus after lung transplantation. Am J Respir Crit Care Med 150:146-52

[36] Dusmet M, Winton TL, Kesten S, Maurer J (1996) Previous intrapleural procedures do not adversely affect lung transplantation. J Heart Lung Transplant 15:249-54

[37] Estenne M, Maurer JR, Boehler A, Egan JJ, Frost A, Hertz M, Mallory GB, Snell Gl, Yousem S (2002) Bronchiolitis obliterans syndrome 2001: an update of the diagnostic criteria. J Heart Lung Transplant 21:297-310

[38] Fein AM, Branman SS, Casaburi R, Irvin CG, Make BJ, Rodarte $J R$, Solway J (1996) Lung volume reduction surgery. This official statement of the American Thoracic Society was adopted by the ATS Board of Directors, May 1996. Am J Respir Crit Care Med 154:1151-2

[39] Gammie JS, Keenan RJ, Pham SM, McGrath MF, Hattler BG, Khoshbin E, Griffith BP (1998) Single- versus double-lung transplantation for pulmonary hypertension. J Thorac Cardiovasc Surg 115:397-402; discussion 402-3

[40] Garrity ER Jr, Mehra MR (2004) An update on clinical outcomes in heart and lung transplantation. Transplantation 77:S68-74

[41] Ghofrani HA, Wiedemann R, Rose F et al. (2002) Sildenafil for treatment of lung fibrosis and pulmonary hypertension: a randomised controlled trial. Lancet 360:895-900

[42] Groetzner J, Wittwer T, Kaczmarek I et al. (2006) Conversion to sirolimus and mycophenolate can attenuate the progression of bronchiolitis obliterans syndrome and improves renal function after lung transplantation. Transplantation 81:355-60

[43] Hamm H (1995) Physiology, pathophysiology and clinical aspects of the pleural cavity. Revision of traditional models. Fortschr Med 113:283-4

[44] Hanson D, Winterbauer RH, Kirtland SH, Wu R (1995) Changes in pulmonary function test results after 1 year of therapy as predictors of survival in patients with idiopathic pulmonary fibrosis. Chest 108:305-10

[45] Haverich A, Aziz S, Scott WC, Jamieson SW, Shumway NE (1986) Improved lung preservation using Euro-Collins solution for flushperfusion. Thorac Cardiovasc Surg 34:368-76

[46] Hoeper MM, Faulenbach C, Golpon H, Winkler J, Welte T, Niedermeyer J (2004) Combination therapy with bosentan and sildenafil in idiopathic pulmonary arterial hypertension. Eur Respir J 24:1007-10

[47] Ikonen T, Kivisaari L, Harjula AL et al. (1996) Value of highresolution computed tomography in routine evaluation of lung transplantation recipients during development of bronchiolitis obliterans syndrome. J Heart Lung Transplant 15:587-95

[48] Jamieson SW, Kapelanski DP, Sakakibara N et al. (2003) Pulmonary endarterectomy: experience and lessons learned in 1,500 cases. Ann Thorac Surg 76:1457-62; discussion 1462-4

[49] Jaramillo A, Smith MA, Phelan D et al. (1999) Temporal relationship between the development of anti-HLA antibodies and the development of bronchiolitis obliterans syndrome after lung transplantation. Transplant Proc 31:185-6

[50] Joyal D, Cantarovich M, Cecere R, Giannetti N (2004) Early experience with two-dose daclizumab in the prevention of acute rejection in cardiac transplantation. Clin Transplant 18:493-6

[51] Keenan RJ, Lega ME, Dummer JS et al. (1991) Cytomegalovirus serologic status and postoperative infection correlated with risk of developing chronic rejection after pulmonary transplantation. Transplantation 51:433-8

[52] Kerem E, Reisman J, Corey M, Canny GJ, Levison H (1992) Prediction of mortality in patients with cystic fibrosis. N Engl J Med 326:1187-91

[53] Keshavjee SH, Yamazaki F, Yokomise H, Cardoso PF, Mullen JB, Slutsky AS, Patterson GA (1992) The role of dextran 40 and potassium in extended hypothermic lung preservation for transplantation. J Thorac Cardiovasc Surg 103:314-25

[54] Khaghani A, Banner N, Ozdogan E et al. (1991) Medium-term results of combined heart and lung transplantation for emphysema. $J$ Heart Lung Transplant 10:15-21

[55] Klepetko W, Mayer E, Sandoval J et al. (2004) Interventional and surgical modalities of treatment for pulmonary arterial hypertension. J Am Coll Cardiol 43:73S-80S

[56] Kotloff RM, Zuckerman JB (1996) Lung transplantation for cystic fibrosis: special considerations. Chest 109:787-98

[57] Lee R, Meyers BF, Sundt TM, Trulock EP, Patterson GA (2002) Concomitant coronary artery revascularization to allow successful lung 
transplantation in selected patients with coronary artery disease. J Thorac Cardiovasc Surg 124:1250-1

[58] Levine SM, Jenkinson SG, Bryan CL, Anzueto A, Zamora CA, Gibbons WJ, Calhoon JH, Trinkle JK (1992) Ventilation-perfusion inequalities during graft rejection in patients undergoing single lung transplantation for primary pulmonary hypertension. Chest 101:401-5

[59] Levine SM, Anzueto A, Peters Jl, Calhoon JH, Jenkinson SG, Bryan CL (1994) Single lung transplantation in patients with systemic disease. Chest 105:837-41

[60] Levy RD, Ernst P, Levine SM et al. (1993) Exercise performance after lung transplantation. J Heart Lung Transplant 12:27-33

[61] Lipson DA, Palevsky HI, Kotloff RM, Edelman J (1998) Conversion to tacrolimus (FK506) from cyclosporine after orthotopic lung transplantation. Transplant Proc 30:1505-7

[62] Martinez JA, Paradis IL, Dauber JH et al. (1997) Spirometry values in stable lung transplant recipients. Am J Respir Crit Care Med 155:285-90

[63] McKenna RJ, Jr., Brenner M, Fischel RJ, Gelb AF (1996) Should lung volume reduction for emphysema be unilateral or bilateral? J Thorac Cardiovasc Surg 112:1331-8; discussion 1338-9

[64] Mendeloff EN, Meyers BF, Sundt TM et al. (2002) Lung transplantation for pulmonary vascular disease. Ann Thorac Surg 73:209-17; discussion 217-9

[65] Meyer DM, Bennett LE, Novick RJ, Hosenpud JD (2001) Single vs bilateral, sequential lung transplantation for end-stage emphysema: influence of recipient age on survival and secondary end-points. J Heart Lung Transplant 20:935-41

[66] Meyer DM, Edwards LB, Torres F, Jessen ME, Novick RJ (2005) Impact of recipient age and procedure type on survival after lung transplantation for pulmonary fibrosis. Ann Thorac Surg 79:950-7; discussion 957-8

[67] Meyers BF, Lynch JP, Battafarano RJ, Guthrie TJ, Trulock EP, Cooper JD, Patterson GA (2000) Lung transplantation is warranted for stable, ventilator-dependent recipients. Ann Thorac Surg 70:1675-8

[68] Morrish WF, Herman SJ, Weisbrod GL, Chamberlain DW (1991) Bronchiolitis obliterans after lung transplantation: findings at chest radiography and high-resolution CT. The Toronto Lung Transplant Group. Radiology 179:487-90

[69] Neumayer HH (2005) Introducing everolimus (Certican) in organ transplantation: an overview of preclinical and early clinical developments. Transplantation 79:S72-5

[70] Niedermeyer J, Bewig B, Bickhardt T et al. (2001) Lung and heartlung transplantation. Guidelines for indications and preoperative diagnosis. Pneumologie 55:396-400

[71] Novick RJ, Andreassian B, Schafers HJ et al. (1994) Pulmonary retransplantation for obliterative bronchiolitis. Intermediate-term results of a North American-European series. J Thorac Cardiovasc Surg 107:755-63

[72] Olschewski H, Simonneau G, Galie N et al. (2002) Inhaled iloprost for severe pulmonary hypertension. N Engl J Med 347:322-9

[73] Orens JB, Estenne M, Arcasoy S et al. (2006) International guidelines for the selection of lung transplant candidates: 2006 update - a consensus report from the Pulmonary Scientific Council of the International Society for Heart and Lung Transplantation. J Heart Lung Transplant 25:745-55

[74] Palmer SM, Miralles AP, Lawrence CM, Gaynor JW, Davis RD, Tapson VF (1999) Rabbit antithymocyte globulin decreases acute rejection after lung transplantation: results of a randomized, prospective study. Chest 116:127-33

[75] Pasque MK, Cooper JD, Kaiser LR, Haydock DA, Triantafillou A, Trulock EP (1990) Improved technique for bilateral lung transplantation: rationale and initial clinical experience. Ann Thorac Surg 49:785-91
[76] Patterson GA, Cooper JD, Goldman B et al. (1988) Technique of successful clinical double-lung transplantation. Ann Thorac Surg 45:626-33

[77] Patterson GM, Wilson S, Whang JL, Harvey J, Agacki K, Patel H, Theodore J (1996) Physiologic definitions of obliterative bronchiolitis in heart-lung and double lung transplantation: a comparison of the forced expiratory flow between $25 \%$ and $75 \%$ of the forced vital capacity and forced expiratory volume in one second. J Heart Lung Transplant 15:175-81

[78] Penn I (1990) Cancers complicating organ transplantation. N Engl J Med 323:1767-9

[79] Penn I (1993) Incidence and treatment of neoplasia after transplantation. J Heart Lung Transplant 12:S328-36

[80] Pham SM, Qi XS, Mallon SM et al. (2002) Sirolimus and tacrolimus in clinical cardiac transplantation. Transplant Proc 34:1839-42

[81] Pochettino A, Kotloff RM, Rosengard BR, Arcasoy SM, Blumenthal NP, Kaiser LR, Bavaria JE (2000) Bilateral versus single lung transplantation for chronic obstructive pulmonary disease: intermediate-term results. Ann Thorac Surg 70:1813-8; discussion 1818-9

[82] Puskas JD, Hirai T, Christie N, Mayer E, Slutsky AS, Patterson GA (1992) Reliable thirty-hour lung preservation by donor lung hyperinflation. J Thorac Cardiovasc Surg 104:1075-83

[83] Quantz MA, Bennett LE, Meyer DM, Novick RJ (2000) Does human leukocyte antigen matching influence the outcome of lung transplantation? An analysis of 3,549 lung transplantations. J Heart Lung Transplant 19:473-9

[84] Reams BD, McAdams HP, Howell DN, Steele MP, Davis RD, Palmer SM (2003) Posttransplant lymphoproliferative disorder: incidence, presentation, and response to treatment in lung transplant recipients. Chest 124:1242-9

[85] Reichenspurner H (2005) Overview of tacrolimus-based immunosuppression after heart or lung transplantation. J Heart Lung Transplant 24:119-30

[86] Reitz BA, Wallwork JL, Hunt SA, Pennock JL, Billingham ME, Oyer PE, Stinson EB, Shumway NE (1982) Heart-lung transplantation: successful therapy for patients with pulmonary vascular disease. N Engl J Med 306:557-64

[87] Revell MP, Lewis ME, Llewellyn-Jones CG, Wilson IC, Bonser RS (2000) Conservation of small-airway function by tacrolimus/cyclosporine conversion in the management of bronchiolitis obliterans following lung transplantation. J Heart Lung Transplant 19:1219-23

[88] Reynaud-Gaubert M, Thomas P, Badier M, Cau P, Giudicelli R, Fuentes $P$ (2000) Early detection of airway involvement in obliterative bronchiolitis after lung transplantation. Functional and bronchoalveolar lavage cell findings. Am J Respir Crit Care Med 161:1924-9

[89] Rubin LJ, Badesch DB, Barst RJ et al. (2002) Bosentan therapy for pulmonary arterial hypertension. N Engl J Med 346:896-903

[90] Saiman L, Mehar F, Niu WW, Neu HC, Shaw KJ, Miller G, Prince A (1996) Antibiotic susceptibility of multiply resistant Pseudomonas aeruginosa isolated from patients with cystic fibrosis, including candidates for transplantation. Clin Infect Dis 23:532-7

[91] Schafers HJ, Haydock DA, Cooper JD (1991) The prevalence and management of bronchial anastomotic complications in lung transplantation. J Thorac Cardiovasc Surg 101:1044-52

[92] Schwartz DA, Van Fossen DS, Davis CS, Helmers RA, Dayton CS, Burmeister LF, Hunninghake GW (1994) Determinants of progression in idiopathic pulmonary fibrosis. Am J Respir Crit Care Med 149:444-9

[93] Seersholm N, Kok-Jensen A, Dirksen A (1994) Survival of patients with severe alpha 1-antitrypsin deficiency with special reference to non-index cases. Thorax 49:695-8

[94] Seoane L, Arcement LM, Valentine VG, McFadden PM (2005) Long-term survival in lung transplant recipients after successful 
preoperative coronary revascularization. J Thorac Cardiovasc Surg 130:538-41

[95] Sharples LD, Tamm M, McNeil K, Higenbottam TW, Stewart S, Wallwork J (1996) Development of bronchiolitis obliterans syndrome in recipients of heart-lung transplantation - early risk factors. Transplantation 61:560-6

[96] Smith CM (1997) Patient selection, evaluation, and preoperative management for lung transplant candidates. Clin Chest Med 18:183-97

[97] Smith MA, Sundaresan S, Mohanakumar T et al. (1998) Effect of development of antibodies to HLA and cytomegalovirus mismatch on lung transplantation survival and development of bronchiolitis obliterans syndrome. J Thorac Cardiovasc Surg 116:812-20

[98] Snell Gl, de Hoyos A, Krajden M, Winton T, Maurer JR (1993) Pseudomonas cepacia in lung transplant recipients with cystic fibrosis. Chest 103:466-71

[99] Snell Gl, Richardson M, Griffiths AP, Williams TJ, Esmore DS (1999) Coronary artery disease in potential lung transplant recipients > 50 years old: the role of coronary intervention. Chest 116:874-9

[100] Snell Gl, Levvey BJ, Zheng L, Bailey M, Orsida B, Law L, Whitford HM, Kotsimbos TC, Williams TJ (2005) Everolimus alters the bronchoalveolar lavage and endobronchial biopsy immunologic profile post-human lung transplantation. Am J Transplant 5:1446-51

[101] Soghikian MV, Valentine VG, Berry GJ, Patel HR, Robbins RC, Theodore J (1996) Impact of ganciclovir prophylaxis on heart-lung and lung transplant recipients. J Heart Lung Transplant 15:881-7

[102] Speich R, Thurnheer R, Gaspert A, Weder W, Boehler A (1999) Efficacy and cost effectiveness of oral ganciclovir in the prevention of cytomegalovirus disease after lung transplantation. Transplantation 67:315-20

[103] Spray TL, Mallory GB, Canter CE, Huddleston CB, Kaiser LR (1992) Pediatric lung transplantation for pulmonary hypertension and congenital heart disease. Ann Thorac Surg 54:216-23; discussion 224-5

[104] Thabut G, Mal H, Castier Y, Groussard O, Brugiere O, MarrashChahla R, Leseche G, Fournier M (2003) Survival benefit of lung transplantation for patients with idiopathic pulmonary fibrosis. J Thorac Cardiovasc Surg 126:469-75

[105] Treede H, Klepetko W, Reichenspurner H, Zuckermann A, Meiser B, Birsan T, Wisser W, Reichert B (2001) Tacrolimus versus cyclosporine after lung transplantation: a prospective, open, randomized two-center trial comparing two different immunosuppressive protocols. J Heart Lung Transplant 20:511-7

[106] Trulock EP (1993) Management of lung transplant rejection. Chest 103:1566-76

[107] Trulock EP, Edwards LB, Taylor DO, Boucek MM, Keck BM, Hertz MI (2006) Registry of the International Society for Heart and Lung Transplantation: twenty-third official adult lung and heart-lung transplantation report 2006. J Heart Lung Transplant 25:880-92

[108] Waddell TK, Bennett L, Kennedy R, Todd TR, Keshavjee SH (2002) Heart-lung or lung transplantation for Eisenmenger syndrome. J Heart Lung Transplant 21:731-7

[109] Walter N, Collard HR, King TE Jr (2006) Current perspectives on the treatment of idiopathic pulmonary fibrosis. Proc Am Thorac Soc 3:330-8

[110] Weill D, Torres F, Hodges TN, Olmos JJ, Zamora MR (1999) Acute native lung hyperinflation is not associated with poor outcomes after single lung transplant for emphysema. J Heart Lung Transplant 18:1080-7

[111] Yankaskas JR, Mallory GB Jr (1998) Lung transplantation in cystic fibrosis: consensus conference statement. Chest 113:217-26

[112] Yonan NA, el-Gamel A, Egan J, Kakadellis J, Rahman A, Deiraniya AK (1998) Single lung transplantation for emphysema: predictors for native lung hyperinflation. J Heart Lung Transplant 17:192-201

\subsection{Interventionelle thorakale Endoskopie}

L. Freitag

\subsubsection{Vorbemerkung}

Die thorakale Endoskopie wird heute in pneumologischen Kliniken zu weit mehr als nur zu diagnostischen Zwecken praktiziert. Bei einigen Erkrankungen der zentralen Atemwege kann man mit endoskopischen Maßnahmen eine Heilung, bei vielen zumindest eine Symptomlinderung für den Patienten erreichen. Die interventionelle Endoskopie des klinisch tätigen Pneumologen umfasst die flexible und starre Bronchoskopie, die medizinische Thorakoskopie und gegebenenfalls die therapeutische Ösophagogastroskopie.

Bei den nachfolgend aufgeführten Prozeduren bestehen erhebliche regionale und institutionelle Differenzen bezüglich der Einschätzung, in wessen Zuständigkeitsbereich die Eingriffe gehören. Vielfach kommt es zu Überschneidungen mit Tätigkeiten, die in einer Nachbarklinik vielleicht nur von operativen Hals-Nasen-Ohrenärzten, Thoraxchirurgen, Gastroenterologen oder Anästhesisten durchgeführt werden. Es ist nicht Ziel dieses Kapitels, die genannten Tätigkeiten grundsätzlich für die Pneumologie zu reklamieren. Prinzipiell sollte im Interesse des Patienten die Prozedur von demjenigen ausgeführt werden, der sie gelernt hat, der sie sicher beherrscht und der sie mit einem entsprechenden Team und ausreichendem Equipment risikoarm durchführen kann.

\subsubsection{Bronchoskopie}

Die Geschichte der Bronchoskopie ist zugleich eine Geschichte der interventionellen Bronchoskopie. Als der Freiburger HNO-Arzt Gustav Killian vor 120 Jahren einen Fremdkörper aus der Luftröhre eines Landwirts entfernte, befreite er den Patienten damit zugleich von seinem quälenden Hustenreiz. In dem Beitrag der Münchner Medizinischen Wochenschrift vom September 1897 »Entfernung eines Knochenstücks aus dem rechten Hauptbronchus auf natürlichem Wege und unter Anwendung der directen Laryngoscopie« wurde erstmals eine interventionelle Prozedur an den Atemwegen beschrieben, die keine operative Eröffnung des Thorax erforderte.

Mit Killians Schule begann die geschickte Kombination von speziell entwickelten Instrumenten wie Rohren, Kathetern und Fasszangen mit elektrischen Lichtquellen. Viele technische Errungenschaften wurden für endoskopische Verfahren modifiziert. Zum heutigen Stand der Technik gehören der Umgang mit Dilatatoren, Mikrodebridern, Ballonkathetern, Lasern, Elektro-, Argonplasma- 
und Kryokoagulationssonden, Iridium-192-Kleinraumbestrahlungssonden, Ultraschallsonden und Platzhaltern. Über den Arbeitskanal des Bronchoskops werden zur Emphysemtherapie Ventile und Stopfen in die Bronchien eingelegt, zur Asthmabehandlung werden glatte Muskelzellen mit Radiofrequenzsonden zerstört und zur Gentherapie von Tumoren werden Vektoren transfiziert.

Untrennbar verbunden mit den technischen Entwicklungen der Endoskopie sind natürlich Weiterentwicklungen der Anästhesieverfahren. Ohne Rachenanästhesie wären selbst einfache Manöver undenkbar. Eine Laserrekanalisation mit anschließender Einlage eines Trachealstents wird schnell und risikoarm nur durch moderne Narkosebeatmungs- und Monitortechniken ermöglicht. 1966 begann der Siegeszug der Fiberbronchoskopie.

Shigeto Ikeda ließ zeitgleich von 2 japanischen Firmen (Machida und Olympus) flexible Endoskope herstellen und entwickelte die wesentlichen Techniken der Inspektion und Probeentnahme. Sein Slogan »More hope with the bronchoscope « erwies sich für eine große Zahl pneumologischer Erkrankungen als richtig. Während heute die diagnostische Bronchoskopie vorwiegend mit flexiblen Videochipgeräten in Lokalanästhesie erfolgt, sind noch immer viele therapeutische Techniken besser, wenn nicht sogar ausschließlich, mit ähnlichen starren Instrumenten durchführbar, wie sie von Killian, Brünings, Kleinsasser und Jackson vor mehr als einem Jahrhundert entwickelt wurden.

\section{Indikationen und Techniken}

Therapeutische Bronchoskopien umfassen ein weites Spektrum von der einfachen Sekretabsaugung auf der Intensivstation über die Entfernung aspirierter Fremdkörper in der pädiatrischen Ambulanz bis zur Implantation von Endoprothesen bei maligner Obstruktion der Trachea. - Tab. 15.13 zeigt die Hauptindikationen für interventionelle Bronchoskopien in einer pneumologischen Klinik.

\section{Aspiration und Sekretverhalt}

Das Mendelson-Syndrom und andere akute Aspirationen sind Indikationen für eine sofortige Absaugung. Nach kurzer Lokalanästhesie sollte mit einem flexiblen Bronchoskop mit großem Arbeitskanal so viel wie möglich abgesaugt werden. Um Zeit zu gewinnen, kann die Anästhesie sehr schnell mit einer transkrikoidalen Injektion von $5 \mathrm{ml}$ Lidocain erfolgen. Finden sich größere Speisereste, muss man gegebenenfalls zur starren Bronchoskopie wechseln. Spülungen jeglicher Art sind obsolet, da man hiermit nur reizendes und möglicherweise bakteriell kontaminiertes Material in die periphereren Atemwege treibt. Es empfiehlt sich, zusätzlich ein $\beta$-Mimetikum (ggf. über das Bronchoskop) zu verabreichen. Damit wird die Hyperreagibilität gedämpft und die mukoziliare Clearance gesteigert.

\begin{tabular}{|c|c|}
\hline \multicolumn{2}{|c|}{$\begin{array}{l}\text { Tab. 15.13. Indikationen und typische bronchoskopische } \\
\text { Prozeduren }\end{array}$} \\
\hline Indikation & Bronchoskopische Prozeduren \\
\hline Sekretverhalt & $\begin{array}{l}\text { Absaugung mit flexiblem } \\
\text { Bronchoskop }\end{array}$ \\
\hline Fremdkörperaspiration & $\begin{array}{l}\text { Entfernung mit Zange oder } \\
\text { Körbchen }\end{array}$ \\
\hline Hämoptysen & $\begin{array}{l}\text { Absaugung, Koagulation, } \\
\text { Bronchusblockade }\end{array}$ \\
\hline Trachealstenose & $\begin{array}{l}\text { Bougierung, Ballondilatation, } \\
\text { Stenteinlage }\end{array}$ \\
\hline Tumorinvasion & $\begin{array}{l}\text { Laser-, Kryo-, Argonplasma-, } \\
\text { Brachytherapie }\end{array}$ \\
\hline Frühkarzinom & $\begin{array}{l}\text { Photodynamische Therapie, } \\
\text { Brachytherapie }\end{array}$ \\
\hline Tumorkompression & Stentimplantation \\
\hline Granulationen & $\begin{array}{l}\text { Mechanische Abtragung, Laser-, } \\
\text { Kryo-, Brachytherapie }\end{array}$ \\
\hline Stumpfinsuffizienz & $\begin{array}{l}\text { Abdichtungsstent, Fibrinklebung, } \\
\text { Spongiosablock }\end{array}$ \\
\hline Ösophagotrachealfistel & Stent- oder Doppelstenteinlage \\
\hline Lungenemphysem & $\begin{array}{l}\text { Bronchusblokade (Ventile), Atem- } \\
\text { wegsbypass (Stents) }\end{array}$ \\
\hline Asthma & $\begin{array}{l}\text { Radiofrequenzablation glatter } \\
\text { Muskelzellen }\end{array}$ \\
\hline
\end{tabular}

Sekretverhalt kommt besonders bei Bronchitikern, in der postoperativen Phase und bei Beatmungspatienten vor. Brodelnde Atemgeräusche, Gasaustauschstörungen und Dystelektasen sind die klinischen und radiologischen Zeichen. Patienten mit diesen Symptomen sollten evtl. sogar mehrmals am Tag fiberoptisch abgesaugt werden. Zeigt das Röntgenbild ein Luftbronchogramm als Ausdruck einer Pneumonie, so profitieren die Patienten selten von einer bronchoskopischen Absaugung. Die gezielte Keimgewinnung kann dennoch eine Indikation für die Bronchoskopie sein. Auch bei Sekretverhalt sind Spülungen kaum sinnvoll. Allenfalls kann bei »mucoid impactions« körperwarme Kochsalzlösung über den Arbeitskanal gegeben werden. Die lokale bronchoskopische Applikation von Mukolytika wie Acetylcystein ist absolut unsinnig und sogar schädlich.

\section{Therapeutische Lavage}

Eine anerkannte Indikation für eine therapeutische Lavage ist die symptomatische Alveolarproteinose. Man kann die Spülungen über den Absaugkanal eines flexiblen Bronchoskops durchführen. Erfahrungsgemäß muss 


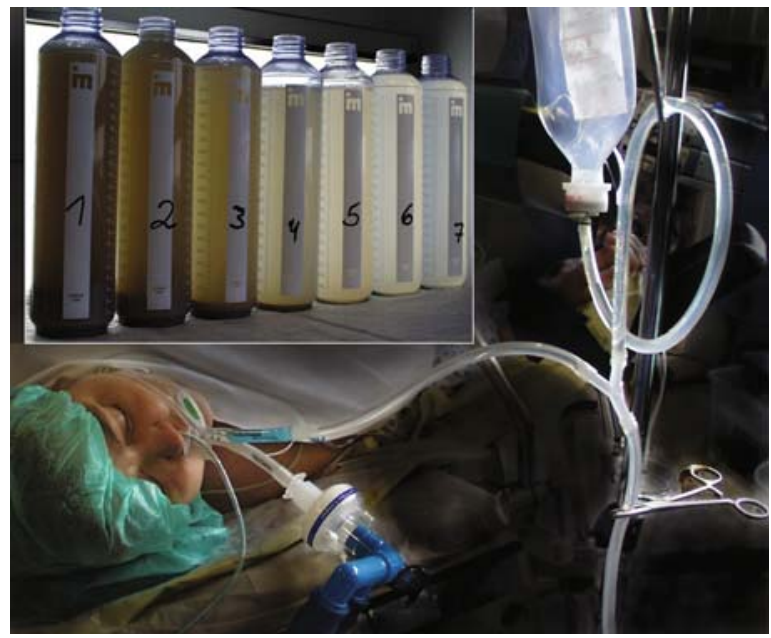

- Abb. 15.24. Therapeutische Lavage bei Alveolarproteinose über einen Doppellumentubus. Nach 20 I Spülung ist die zurückgewonnene Flüssigkeit ausreichend klar

man pro Segment mit ca. $300 \mathrm{ml}$ lavagieren. Dabei orientiert man sich an der Trübung (und falls möglich der gemessenen Extinktion) der zurückgewonnenen Flüssigkeit. Bei ausgeprägtem Krankheitsbild ist es aber effizienter, den Patienten mit einem Doppellumentubus $\mathrm{zu}$ intubieren und den exzessiven Surfactantüberschuss aus zunächst einem Lungenflügel auszuwaschen. Oft braucht man mehr als 201 physiologische Kochsalzlösung, bis die Flüssigkeit klar genug zurückkommt (- Abb. 15.24). Einige Tage später kann die Lavage der anderen Seite erfolgen.

\section{Fremdkörperaspiration}

Viele, aber eben nicht alle Fremdkörper sind röntgenschattengebend. Besonders bei Kindern, die hartnäckig husten, Pneumonien entwickeln oder über längere Zeit eitrigen Auswurf haben, muss man auch bei normalem Röntgenbild an aspirierte Nüsse, Legosteine und Ähnliches denken. Es gibt viele Debatten, ob Fremdkörper immer starr entfernt werden sollten, oder ob man es zunächst flexibel versucht. Prinzipiell sollte man eine Extraktion nur vornehmen, wenn man entsprechende Fasszangen, Schlingen oder eine Krysonde zur Hand hat. Dabei muss man unbedingt vermeiden, einen Fremdkörper, den man nicht richtig fassen kann, noch tiefer in einen Segmentbronchus zu treiben.

Spitze, scharfkantige und spießende Gegenstände sollte man, auch wenn man viel Erfahrung besitzt, nur in Narkose mit starrem Instrumentarium entfernen. Eine Bergung ist möglich, selbst wenn der Fremdkörper sehr groß ist. So kann man z. B. eine Nadel mit der optischen Zange fassen und zunächst bis an das distale Ende des Bronchoskoprohrs heranziehen. Nun kann man ohne
Gefahr der Spießverletzung mit der gefassten Nadel im "Windschatten« des Bronchoskops extubieren.

Schwierig ist die Situation bei chronischen, eingewachsenen Fremdkörpern. Wegen der Perforationsgefahr sollte der bronchologische Eingriff nur in OP-Bereitschaft erfolgen. Man darf keinen falschen Ehrgeiz entwickeln. Eine weitere Gefahr besteht, wenn man einen großen Fremdkörper, um den sich schon Granulationsgewebe gebildet hat, zwar in die Trachea zurückziehen kann, ihn aber nicht durch die Stimmbandebene bringt. Falls er in diesem Augenblick zurückfällt, kann er in die zuvor nicht befallenen Bronchusabschnitte rutschen und diese verlegen. Es besteht die Gefahr einer Bolusasphyxie. Sicherheitshalber sollten die Patienten daher mit der befallenen Seite nach unten gelagert werden.

\section{Hämoptysen}

Fädchenförmige Blutbeimengungen im Sputum werden als Hämoptysen, das Abhusten von reinem Blut als Hämoptoe bezeichnet. Die Ursachen sind vielfältig und können durch Anamnese und Röntgenbefund meistens eingegrenzt werden. Die Gefahr bei einer Hämoptoe ist nicht der Blutverlust und eine damit verbundene Kreislaufdepression, sondern das mögliche Verstopfen der Atemwege mit Blut und Koageln (auch der primär nicht befallenen Lungenlappen) und die daraus resultierende Asphyxie.

Die Erstmaßnahmen müssen daher darauf ausgerichtet sein, den Gasaustausch aufrecht zu erhalten. Der Patient mit Bluthusten sollte als erstes eine Sauerstoffsonde bekommen und mit dem Oberkörper aufgerichtet oder, sofern die Blutungsquelle bekannt ist, mit der blutenden Seite nach unten halbschräg gelagert werden. Man verhindert damit zumindest teilweise, dass Blut in die gesunden Lungenareale läuft, sobald der Hustenreflex ausgeschaltet ist.

Bei einer massiven Hämoptoe sollte starr bronchoskopiert werden. Als Alternative kann eine Tubusbronchoskopie erfolgen. Über einen bronchoskopisch eingelegten 8,5er- oder 9er-Tubus kann man auch größere Blutmengen und Koagel entfernen, die nicht durch den Arbeitskanal eines Fiberbronchoskops abgesaugt werden können. Zunächst muss man die Atemwege so schnell wie möglich freisaugen, um die Hypoxämie zu verhindern. Hat man genügend Übersicht gewonnen und ist der Patient stabil genug, kann man eine Blutstillung versuchen.

Bei einer iatrogenen Blutung, z. B. nach transbronchialer Biopsie, kann man das sogenannte Zavala-Manöver versuchen. Das Bronchoskop wird in dem Bronchus, aus dem es blutet, in Wedge-Position gebracht und nun für mehrere Minuten maximal gesaugt. Der erzeugte Segmentbronchuskollaps führt fast immer zum Sistieren der Blutung.

Blutet es diffus, kann man eiskalte Kochsalzlösung oder vasokonstriktive Medikamente über den Kanal des Fiberbronchoskops geben. Adrenalinlösung ist wegen der $\beta$-stimulierenden Wirkung weniger sinnvoll als das mehr 
a-stimulierende Noradrenalin. Infrage kommen auch verdünnte Lösungen von Otriven ${ }^{\circledR}$, Privin ${ }^{\circledR}$ oder POR 8. Vasopressin (Glycilpressin ${ }^{\circledast}$ ) ist am effektivsten, aber auch bei weitem am teuersten. Keinesfalls darf man wie im Gastrointestinaltrakt sklerosierende Substanzen in die Bronchusschleimhaut injizieren. Etoxysklerol kann beispielsweise eine Nekrose der Schleimhaut und sogar der Knorpel bewirken.

Bei Blutungen aus sichtbaren Tumoren oder Hämangiomen kann man mit dem Laser oder dem Elektrokauter koagulieren. Blutet es stärker, ist der Laser ungeeignet, da das Blut die Energie absorbiert, ohne dass man das zuführende Gefäß veröden kann. Ideal ist für diese Fälle die Argonplasmakoagulation (APC). Die APC wirkt im Vergleich zum Laser nur sehr oberflächlich. Da der HF-Strom aber dem Weg des geringsten elektrischen Widerstands folgt und Blut und Blutgefäße gegenüber Normalgewebe eine geringere Impedanz haben, ist die APC auch wirksam, wenn die Blutungsquelle nicht genau lokalisiert werden kann. Das preisgünstigste Verfahren ist noch immer die HF-Elektrokoagulation. Alle in der Chirurgie üblichen Elektrokautergeräte können verwendet werden, es sind aber elektrisch isolierte Fiberbronchoskope erforderlich.

Auch eine Brachytherapie mit 5-8 Gy ist innerhalb weniger Tage hämostyptisch wirksam. Bei Hämoptysen aus zentralen Tumoren kann es daher sinnvoll sein, eine endoluminale Kleinraumbestrahlung einer perkutanen Strahlentherapie voranzustellen.

Blutet es sehr stark, muss eine Tamponade erfolgen. Bei einer starren Bronchoskopie schiebt man das Rohr in den nicht befallenen Hauptbronchus, saugt ab und bewahrt den Lungenflügel für den Gasaustausch. Alternativ können Haupt- oder Lappenbronchien mit Tupfern tamponiert werden. Für die fiberbronchoskopische Tam- ponade bei schwerer Hämoptoe kann ein gewöhnlicher dünner Tubus oder besser ein überlanger Spezialtubus (Bronchosafe) in den kontralateralen Hauptbronchus eingelegt werden. Auf diese Weise wird jedoch ein gesamter Lungenflügel von der Ventilation abgeschnitten. Eleganter ist die Verwendung eines Bronchusblockers, besonders wenn die Blutungsquelle bis auf Lappenbronchusebene lokalisiert werden kann.

Der Ballonkatheter kann, falls notwendig mehrere Tage belassen werden. Das schafft Zeit, um beispielsweise eine Bronchialarterienembolisation oder eine elektive Operation vornehmen zu können. Sind die Verhältnisse völlig unübersichtlich, bleibt nur noch die Intubation mit einem Doppellumentubus (z. B. Carlenstubus). Während man beide Seiten getrennt beatmet und zumindest verhindert, dass Blut von einer zur anderen Seite gelangt, hat man eine realistische Chance, den Patienten ohne Asphyxie in den Operationssaal zu bringen.

Wann ein Patient thorakotomiert werden soll, muss vom Gesamtzustand und von der Grunderkrankung abhängig gemacht werden. In einigen Fällen kann die interventionelle Radiologie mit Embolisationen von Tumorgefäßen, Gefäßkonvoluten oder Ähnlichem eine weniger belastende Alternative sein, sofern mit bronchologischen Maßnahmen die akute Lebensgefahr abgewendet werden kann.

\section{Obstruktionen der zentralen Atemwege}

Obstruktionen der großen Atemwege lassen sich nach verschiedenen Gesichtspunkten einteilen. Für allgemeine Behandlungen ist es natürlich sinnvoll, zwischen malignen und benignen Verengungen zu unterscheiden. Neben dem Verengungsgrad bestimmt die Lokalisation, insbesondere die Frage, ob der singuläre Atemweg Trachea
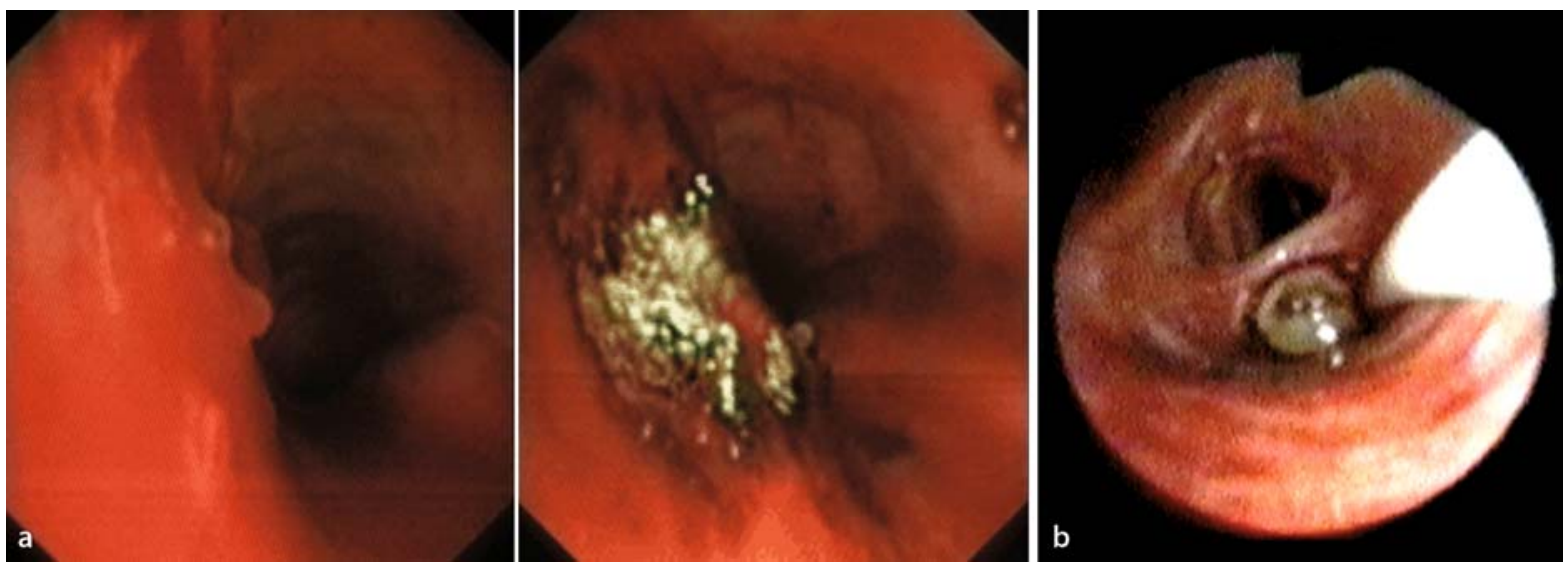

- Abb. 15.25a,b. a Bei einer sichtbaren Blutungsquelle kann man mit dem Argonplasmakoagulator oder dem Laser die Blutung stillen. b Um eine unstillbare Blutung aus einem Lappenbronchus zu beherrschen, kann eine Tamponade mit einem fiberbronchoskopisch platzierten
Bronchusblocker erfolgen. Der Blockerballon kann notfalls einige Stunden oder sogar Tage belassen werden, z. B. um den Patienten für einen operativen Eingriff zu stabilisieren 
betroffen ist oder nicht, die Dringlichkeit des Handelns. Unter biomechanischen Aspekten und für die Planung endoskopischer Maßnahmen sollte man 4 Formen von Atemwegsverengungen unterscheiden:

1. intraluminal, exophytisch wachsende Gewebsvermehrungen (Tumore, Granulationen),

2. von außen komprimierende Prozesse (Lymphome),

3. narbige Strikturen und

4. Dyskinesien (Malazie, schlaffe Hinterwand).

In unseren pneumologischen Kliniken erfolgen die meisten endoskopischen Eingriffe wegen maligner Erkrankungen. Weitere Indikationen sind narbige Trachealstenosen, meistens als Folgen vorausgegangener Intubationsbeatmungen, und eher selten postentzündliche Verengungen (TBC, Sarkoidose, Morbus Wegener usw.).

\section{Rekanalisationstechniken}

Die Tumorobstruktion ist eine der quälendsten Manifestationen des zentralen Bronchialkarzinoms. Die endoskopische Therapie muss sich nach Grad und Art der Tumorausbreitung richten. Vorrangiges Ziel ist die Verbesserung der Lebensqualität (meistens Linderung der Luftnot) mit möglichst kurzer Hospitalisierungszeit des Patienten. Prinzipielle Empfehlungen zur Durchführung der einzelnen Maßnahmen wurden von der Deutschen Gesellschaft für Pneumologie und von der Sektion Endoskopie erarbeitet. Es gibt international anerkannte Empfehlungen der ATS und der ERS.

\section{Laser}

Exophytisch wachsende Tumore in der Trachea und den großen Bronchien kann man endoskopisch verkleinern oder abtragen. Neben der mechanischen Abtragung mit dem starren Bronchoskop (»coring out«) haben sich verschiedene Verfahren mit gleichzeitiger Blutstillung bewährt. Am gezieltesten arbeitet man mit dem Laser.

Die HNO-Ärzte arbeiten vorwiegend mit Spiegelkonstruktionen und dem $\mathrm{CO}_{2}$-Laser bei $10.600 \mathrm{~nm}$. Der Vorteil dieses Lasers ist die geringe Eindringtiefe, sodass das Risiko tieferer Gewebsschäden z. B. an den Stimmbändern vermieden wird. Seine Koagulationswirkung ist aber für endobronchiale Anwendungen unzureichend.

Zwar gibt es inzwischen verschiedene Diodenlaser, aber die größte Verbreitung in der Bronchologie und der Gastroenterologie hat noch immer der ND-YAG-Laser (- Abb. 15.26a). Er arbeitet bei $1064 \mathrm{~nm}$ im nahen Infrarotbereich. Das energiereiche Licht wird über flexible Glasfasern meistens über den Arbeitskanal eines Fiberbronchoskops geleitet. Für bronchoskopische Anwendungen reicht ein luftgekühltes Gerät mit $40 \mathrm{~W}$ Leistung aus. Es gibt verschiedene Laserspitzen, sodass man entweder im Kontaktmodus oder berührungslos mit einigen Millimetern Abstand arbeitet. Bei niedriger Energie um $20 \mathrm{~W}$ (bis $1000 \mathrm{~J}$ ) lassen sich auch stark vaskularisierte Exophyten sicher bis zu einer Tiefe von $10 \mathrm{~mm}$ koagulieren.

Der Effekt auf das Gewebe ist abhängig vom Abstand der Laserspitze, von der eingestellten Energie, von der Pulsdauer und von den Absorptions- und Streuungseigenschaften des Gewebes. Vaskularisierte Prozesse absorbieren stärker als blasses Gewebe. Bei größeren Energien karbonisiert und verdampft das Gewebe und man kann auf diese Weise bei niedriger Blutungsgefahr Tumore allein und vollständig mit dem Laser abtragen. Dabei wird allerdings viel reizender Rauch und Schmauch freigesetzt, der bei kaum vermeidbarer Rückatmung Entzündungsreaktionen der Lunge zur Folge haben kann. Die unvermeidbare thermische Reaktion am umgebenden gesunden Gewebe birgt zudem die Gefahr sekundärer Gewebsschäden. Lange Laserimpulse können dazu führen, dass glühende Gewebsteilchen gegen die Schleimhaut spritzen (Popcorn-Effekt). Auch sollte die inspiratorische Sauerstoffkonzentration während der Laserapplikation unter 0,5 gehalten werden, um Verpuffungen und Verbrennungen zu vermeiden.

Die meisten Endoskopiker koagulieren die Tumoren bis sie schrumpfen und räumen anschließend das denaturierte Gewebe mit Fasszangen aus. Dieses Verfahren hat sich als sicher und schnell in den meisten europäischen Ländern durchgesetzt. In Amerika wird vorwiegend fiberoptisch in Lokalanästhesie gearbeitet und auf mechanische Ausräumung verzichtet. Diese Tumorverkleinerungen dauern wesentlich länger. Eine endoskopische und klinische Response auf den Lasereingriff ist in ca. 75\% der Fälle zu erwarten. Patienten, bei denen eine Wiedereröffnung gelingt, leben beschwerdefreier und im Mittel 4 Monate länger.

\section{Argonplasmakoagulation}

Argonplasmakoagulatoren und Elektrokauter können ebenfalls zur Rekanalisation verwendet werden. Ihnen ist gemeinsam, dass das Gewebe mit einem Hochfrequenzstrom koaguliert und geschnitten wird. Während man bei der gewöhnlichen Elektrokoagulation eine leitende Sonde oder eine Schlinge an den Tumor legen muss, arbeitet man mit der APC berührungslos (• Abb. 15.26d). Über einen millimeterdicken Argongasstrahl gelangt die Energie auf das Gewebe. Im Gegensatz zum Laser geht dabei der Strahl nicht immer geradeaus sondern je nach elektrischem Widerstand (Wassergehalt, Blutgehalt) auch »um die Ecke«. Dies ist etwas gewöhnungsbedürftig und man kann nicht so sauber schneiden wie mit dem YAG-Laser, andererseits kann man mit dem APC auch in schwer erreichbaren Regionen (Oberlappenbronchus) und wie erwähnt bei stärkeren Blutungen arbeiten.

Das Gewebe verblasst und verdampft innerhalb von Sekunden. Die Eindringtiefe richtet sich nach der eingestellten Energie und der Zeit, sie ist in jedem Fall auf 
weniger als $5 \mathrm{~mm}$ begrenzt. Um größere Gewebemassen abzutragen, muss man daher Schicht für Schicht koagulieren und mechanisch ausräumen. Es gibt verschiedene APC-Sonden für starre und für flexible Bronchoskope mit und ohne Absaugkanal. APC-Geräte sind leicht transportabel, preisgünstig und sehr universell einsetzbar, z. B. für gastrointestinale Blutstillungen.

\section{Kryotherapie}

Die Kryotherapie (-Abb.15.26b) ist neben der Elektrokoagulation und der APC eine weitere preisgünstige Alternative zum Laser. Bei der Kryotherapie wird durch Entspannung von flüssigem Lachgas (Joule-Thomson-Effekt) eine Sondenspitze innerhalb weniger Sekunden auf bis $\mathrm{zu}-89^{\circ} \mathrm{C}$ abgekühlt. Die Sonde bringt man durch ein

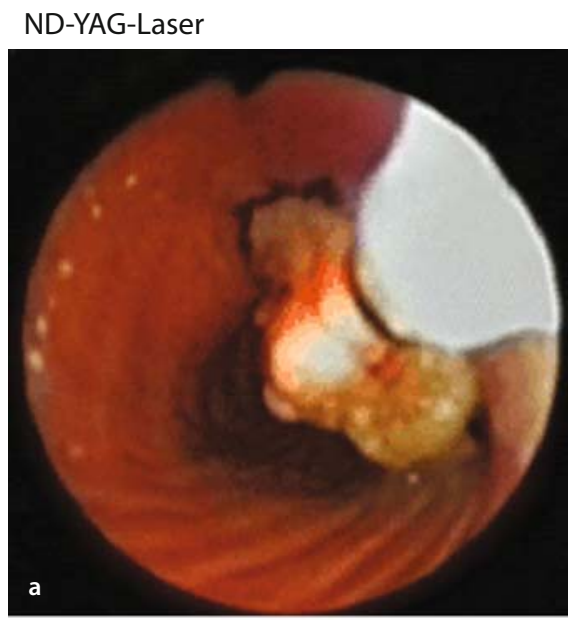

PDT-Laser

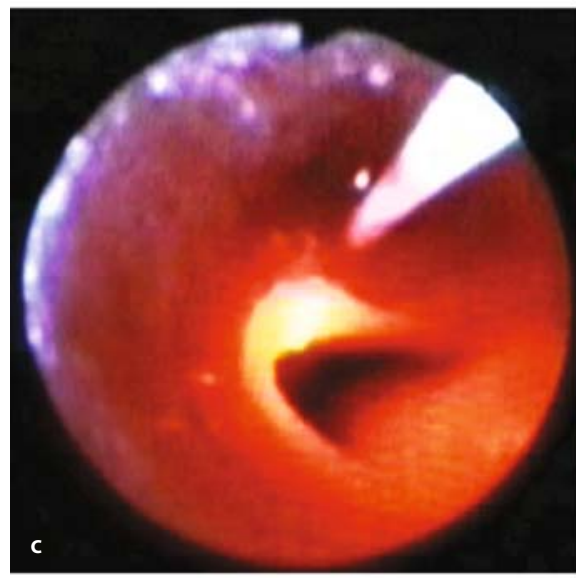

Stent

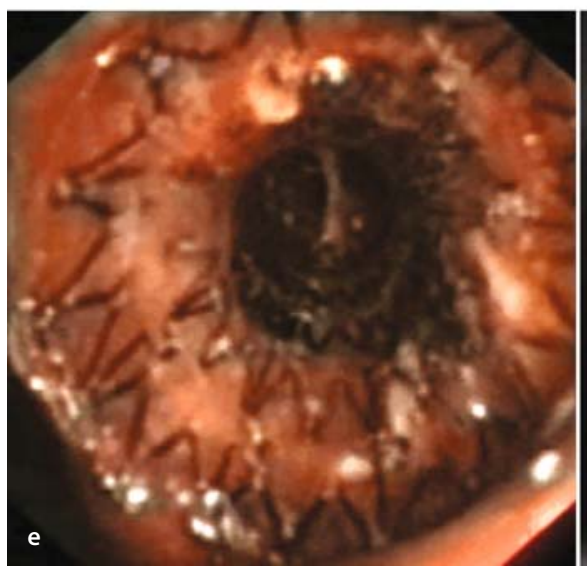

Kryosonde

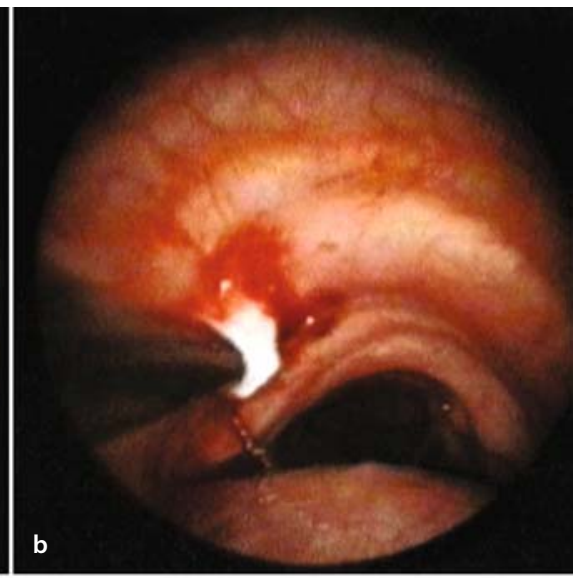

Argon-Plasma-Koagulator

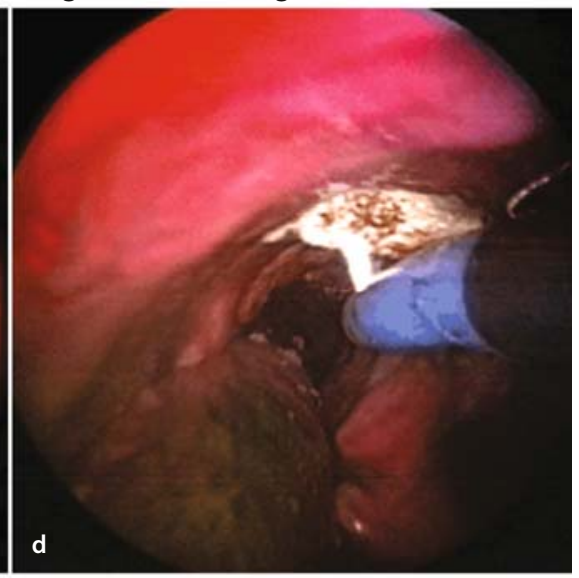

Brachytherapie

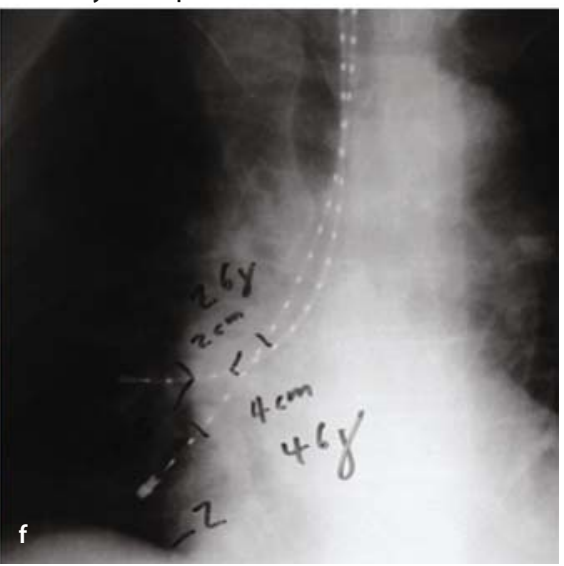

- Abb. 15.26a-f. Verfahren zur endoluminalen Abtragung von Fremdgewebe. a ND-YAG-Laser, b Kryotherapie, c photodynamische Therapie, d Argonplasmakoagulation. e Zur Behandlung von Kompressionen benutzt man Stents. f Intramurale Tumore erreicht die Brachytherapie 
flexibles oder starres Endoskop in oder an einen Tumor heran. In mehreren Vereisungs- und Auftauzyklen wird das Gewebe denaturiert. Da keine Entzündungsgefahr besteht, kann man auch in unmittelbarer Nähe von entflammbaren Fremdkörpern oder Stents arbeiten.

Die Kryotherapie hat zudem einen gewissen selektiven Gewebseffekt. Tumorzellen und Granulationen sind empfindlicher als gesundes Epithel, Knorpel ist relativ kälteresistent. Dies macht die Kryotherapie zu einem sicheren Verfahren, sofern man ausreichend Zeit hat. Im Vergleich zu einem Eingriff mit dem Laser oder dem APC dauert die Kryotherapie deutlich länger. Mehrere Vereisungszyklen von jeweils $2 \mathrm{~min}$ sind notwendig. Bei großen Tumoren in der Trachea mit drohender Asphyxie ist das Verfahren daher weniger geeignet. Dies gilt auch für stark vaskularisierte Prozesse. Das strömende warme Blut verhindert ein ausreichendes Gefrieren und damit die gewünschte Gewebszerstörung.

In manchen Situationen kann man sich einen $\mathrm{Ne}$ beneffekt der Kryotherapie, nämlich den Klebeeffekt der Kälte zunutze machen. Gewebsteile, Koagel oder auch Fremdkörper, die schwer mit Zangen zu greifen sind, können nach oberflächlicher Berührung und Anfrieren an der Sonde aus dem Bronchialsystem entfernt werden.

Bei malignen Tumoren ist die Effizienz bezüglich Symptomlinderung und Lebenszeitverlängerung von Kryotherapie und Laser vergleichbar.

Kombinationen mit externer Strahlentherapie zeigen Vorteile gegenüber alleiniger Strahlentherapie. Die Vorund Nachteile der einzelnen Verfahren sind nicht sehr gravierend. Der guten Schneidewirkung des Lasers stehen die einfachere Blutstillung des Argonplasmakoagulators und die relative Knorpelschonung der Kryotherapie gegenüber. Erfahrene Endoskopiker können in den allermeisten Fällen mit jeder der genannten Methoden eine ausreichende Tumorverkleinerung erreichen.

\section{Photodynamische Therapie}

Auf einem gänzlich anderen Wirkmechanismus beruht die photodynamische Therapie (PDT; • Abb. 15.26c). Eine PDT erfolgt in 3 Schritten. Zunächst wird ein Sensitizer intravenös oder als Aerosol gegeben. Der Sensitizer akkumuliert im Tumor oder wird verzögert ausgeschieden. Wegen seiner Fluoreszenzeigenschaften kann man ihn spektrometrisch detektieren oder mit entsprechenden Filtern sichtbar machen. Diese sogenannte photodynamische Diagnostik (PDD) kann zur Frühdiagnostik von Bronchialkarzinomen und zur Bestimmung von Tumorgrenzen benutzt werden. Nach maximaler Anreicherung des Sensitizers im Tumor erfolgt eine intensive Lichtbestrahlung meistens mit einem Farblaser. Durch einen photodynamischen Effekt wird beim Zerfall des Sensitizers zytotoxischer Singuletsauerstoff frei, der den Tumor innerhalb von wenigen Tagen in die Apoptose oder
Nekrose bringt und die Tumorgefäße infarzieren lässt. Im dritten Schritt wird das abgestorbene Gewebe mechanisch ausgeräumt (Debridement).

In Deutschland ist für die Behandlung des Bronchialsystems bisher nur das Photofrin ${ }^{\circledR}$ zugelassen. Dieses Hämatoporphyrinderivat wird intravenös verabreicht. Es gibt praktisch keine systemischen Medikamentenreaktionen bzw. Nebenwirkungen und das Photofrin wird rasch verstoffwechselt und ausgeschieden. Die Clearance aus der Haut ist jedoch verzögert. Dies führt unvermeidbar zu einer kutanen Lichtempfindlichkeit, die so ausgeprägt ist, dass die Patienten für mehrere Wochen direktes Sonnenlicht meiden müssen. 2 Tage nach Medikamentengabe wird der Tumor mit rotem Licht von $630 \mathrm{~nm}$ über ca. 8 min beleuchtet. Hierzu werden heute Diodenlaser verwendet. Bei den typischen Energien im Prozentbereich eines YAG-Lasers kommt es nicht zu einem erkennbaren thermischen Effekt sondern zu einer rein phototoxischen Reaktion mit verzögertem Zelltod des Tumorgewebes.

Gegenüber allen anderen Verfahren wirkt die PDT wesentlich selektiver auf Tumorzellen und schont das Normalgewebe. Die Selektivität und die Stärke der zytotoxischen Reaktion hängen dabei von der Anreicherung des Sensitizers im Tumor sowie von der Qualität der Ausleuchtung ab. Neuere Sensitizer wie m-THPC (Foscan ${ }^{\circledR}$ ), Chlorin-6 (Fotolan ${ }^{\circledR}$ ) oder SnET2 (Purlytin ${ }^{\circledR}$ ) sind potenter bezüglich der photochemischen Reaktion (»quantum yield«) und sie werden schneller aus der Haut geklärt. Da sie bei Wellenlängen über $650 \mathrm{~nm}$ absorbieren, ist die Eindringtiefe höher. Derzeit sind diese Substanzen für intrathorakale Anwendungen aber nicht zugelassen und stehen nur im Rahmen von Studien zur Verfügung.

Insgesamt ist die photodynamische Therapie exophytischer Tumore sehr aufwendig und teuer. In Vergleichsstudien erwies sich die PDT als etwas sicherer und es zeigten sich Überlebenszeitvorteile gegenüber der konventionellen Lasertherapie. Der hohe apparative Aufwand, die hohen Kosten des Sensitizers und die kutane Lichtempfindlichkeit stehen aber einer Verbreitung der Methode entgegen. Sequenzielle Therapien mit Nd-YAG-Laser plus PDT sowie PDT plus Brachytherapie erwiesen sich auch in unserem Krankengut allen Einzeltherapien überlegen. Sie sind aber maximal aufwendig und müssen auf wenige Patienten mit kurativem Ansatz beschränkt bleiben.

\section{Brachytherapie}

Bei vorwiegend intramuralem Tumorwachstum nichtkleinzelliger Karzinome und zur Stabilisierung eines Rekanalisationserfolgs ist die Brachytherapie das Verfahren der Wahl (• Abb. 15.26f). Die Hochdosiskurzzeittherapie mit ${ }^{192}$ Iridium hat sich in Deutschland und den meisten europäischen Ländern durchgesetzt.

In Lokalanästhesie wird meistens in Seldinger-Technik ein Katheter in den tumorbefallenen Bronchusabschnitt 
eingelegt. Nach Röntgenkontrolle der Katheterlage wird hierdurch über wenige Minuten mit einer von einem Schrittmotor geführten Iridiumquelle bestrahlt. Es können mehrere Sonden eingelegt werden, um multilokuläre Tumore zu bestrahlen. Regime mit 4-mal 5 Gy oder 3-mal 7,5 Gy in Wochenabständen (bezogen auf $10 \mathrm{~mm}$ von der Strahlenachse) werden von den meisten Zentren empfohlen. Über die optimale Dosisverteilung und die Intervalle wird kontrovers diskutiert. So erwies sich die zweimalige Gabe von 7,2 Gy im Abstand von 3 Wochen als ebenso wirksam wie eine 4-malige Applikation von 3,8 Gy in Wochenabständen. Bei geringerem Aufwand sind die Nebenwirkungen nicht vermehrt. Die Brachytherapie wird im Allgemeinen erst nach einer perkutanen Strahlentherapie durchgeführt. Gegenüber alleiniger Strahlentherapie verbessert ein Boost mit endoluminaler Kleinraumbestrahlung die Palliation.

Die gefürchtetste Nebenwirkung ist die Hämoptoe. Die meisten Daten sprechen aber dafür, dass die Hämoptoe wohl eher dem natürlichen Krankheitsverlauf bei verlängerter Überlebenszeit zuzuschreiben ist als der Nebenwirkung der Brachytherapie. Ein erhöhtes Risiko besteht, wenn der Kleinraumbestrahlung unmittelbar eine Lasertherapie vorangegangen ist. Tumorverkleinerung und hämostyptische Bestrahlung sind die Hauptindikationen, die Brachytherapie kann aber auch eingesetzt werden, um das Nachwachsen überschießender Granulationen z. B. an Stentkanten oder nach Verbrennungstraumen zu verhindern.

\section{Starr oder flexibel?}

Laser-, Kryo-, Elektro- und fotodynamische Therapie können mit dem Fiberbronchoskop durchgeführt werden. In starrer Technik mit Vollnarkose kann man jedoch präziser und schneller arbeiten. Dank moderner Anästhesieverfahren sind die Risiken für den Patienten minimal und mit großen Fasszangen kann man in jedem Fall schneller Fremdgewebe ausräumen als mit dem Instrumentarium der flexiblen Endoskopie. Es sind signifikant weniger Eingriffe erforderlich. Sofern man Fiberglasinstrumente in Lokalanästhesie einsetzt, muss man die starre Endoskopie vorhalten, um bei Komplikationen wechseln zu können. Nach jeder endoskopischen Gewebsabtragung und Koagulation muss zeitnah eine Kontrollbronchoskopie mit Debridement durchgeführt werden, da Verlegungen z. B. durch Fibrinausschwitzungen erwartet werden müssen. Brachytherapien lassen sich problemlos in Lokalanästhesie durchführen.

\section{Dilatation und Stents}

Schrumpfende, narbige Prozesse wie Anastomosenstrikturen kann man mit Bougies oder Angioplastiekathetern aufdehnen. Die Dilatation sollte langsam und vorsichtig über mehrere Minuten vorgenommen werden. Es empfiehlt sich, den Ballonkatheter mit Kontrastmittel zu füllen und während der Aufdehnung zu durchleuchten. Man erkennt dann, wie die Schnürtaille langsam verstreicht. In einigen Fällen erreicht man durch Dilatation einen lang anhaltenden Effekt, bei anderen Patienten kommt es in Tagesfrist wieder zu einer neuen Einschnürung. Prädiktoren, ob eine Bougierung Erfolg haben wird, sind nicht bekannt. Das relativ sichere und kostengünstige Manöver kann aber versuchsweise erfolgen bevor man sich zu invasiveren Maßnahmen entschließt.

Dominiert die extrabronchiale Kompression (z.B. durch Lymphknoten) oder liegt eine weitgehende Wandzerstörung vor, besteht die Indikation zur Stenteinlage. Es gibt sehr viele verschiedene Stents. Viele Stents waren kommerziell erhältlich oder wurden in Studien geprüft. Nur wenige Modelle haben aber den Test der Zeit überstanden.

Für die obere Trachea nahe der Stimmbänder empfiehlt sich noch immer der Montgomery-T-Stent. Eine Tracheotomie ist erforderlich, aber die Gefahr einer Stimmband- oder Rekurrensnervschädigung ist minimal. Die Patienten können sich problemlos selbst absaugen und bei Bedarf Sauerstoff über den T-Schenkel zuführen.

Die größte Verbreitung weltweit hat der DumonSilikonstent (Vertrieb Novatech). Die zylinderförmigen Stents sind in verschiedenen Durchmessern und Längen erhältlich. Sie können bei allen Arten von Kompressionen, Tumorinvasionen und Strikturen auf Tracheal- und Bronchusniveau eingesetzt werden. Kleine Noppen an der Außenseite verhindern weitgehend die Dislokationen. Bei Malazien sind sie ungeeignet, da sie nur durch Anpressdruck vor Ort gehalten werden. Die Dumon-Stents müssen mit starren Bronchoskopen eingelegt werden. Sie können jederzeit repositioniert oder entfernt werden. Eine regelmäßige Feuchtinhalation wirkt der häufigsten Nebenwirkung der Stents, der Mukostase, entgegen.

Eine Alternative zu den Dumon-Stents sind die Polyflex-Stents (Boston Scientific). Sie sind ebenfalls aus Silikon, sind aber dünnwandiger und beziehen ihre Rückstellkraft aus inkorporierten Trevirafäden. Polyflex-Stents können sich sanduhrförmigen Stenosen besser anpassen. Die Dislokationsgefahr ist größer als bei den Dumon-Stents.

Für den Bifurkationsbereich und für lange Trachealstenosen hat sich der Y-förmige dynamische Stent (Boston Scientific) bewährt. Er ist anatomisch geformt und hat eine flexible Hinterwand, die die Hustenclearance verbessert. Modelle in 3 Größen sind erhältlich, die Stents können bedarfsweise auf die ideale Länge geschnitten werden. Auch der dynamische Stent wird mit starrer Bronchoskopie eingelegt. Er kann praktisch nicht dislozieren und lässt sich wie die vorgenannten Stents jederzeit problemlos entfernen.

Neben den Silikonmodellen gab es verschiedenste Metallstents. Heute werden fast ausschließlich Modelle verwendet, die zum größten Teil oder vollständig mit einem dünnwandigen Kunststoff ummantelt sind. Sie sind inzwischen alle selbstexpandierend und werden über ei- 
nen Führungsdraht von einem semirigiden Träger in der Stenose freigesetzt. Dabei wird der Freisetzungsprozess über das flexible Bronchoskop und oder eine Durchleuchtung kontrolliert. Besonders verbreitet sind die UltraflexStents (Boston Scientific). Diese selbstexpandierenden Nitinolgeflechte sind relativ gewebefreundlich und passen sich auch irregulären Bronchusstrukturen gut an. Man muss aber damit rechnen, dass sich an den unbeschichteten Kanten Granulationsgewebepolypen bilden können. Bei Patienten, die oft und stark husten, beobachtet man häufiger Ermüdungsbrüche der Drahtstents.

Ultraflex-Stents ändern ihre Länge abhängig vom Kompressionszustand. Während der Einlage verkürzen sie sich um ein Drittel. Neuere Stents wie der AeroStent (Alveolus) sind längenkonstant. Die Kanten diese Stents irritieren auch die Schleimhaut geringer, sodass weniger Granulationen auftreten. Auch bei den HanaroStents (Vertrieb MTW) haben wir bisher keine relevanten Granulationen beobachtet. Inzwischen gibt es Firmen die neben Standardprodukten individuell gefertigte Niti-

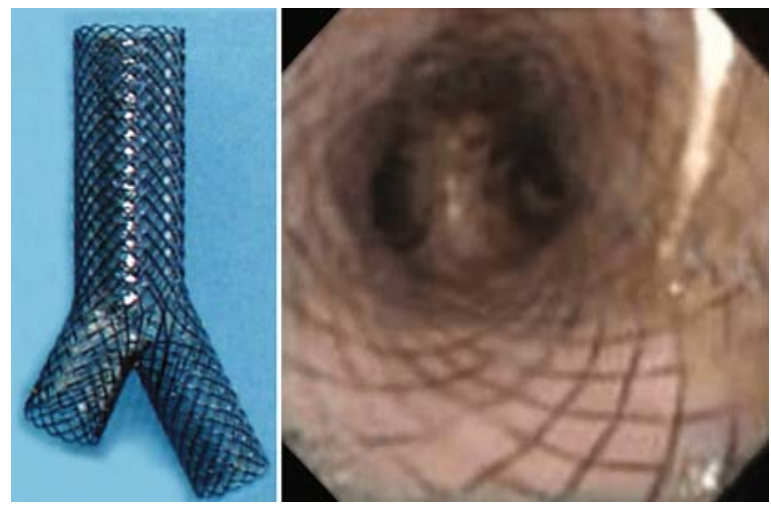

- Abb. 15.27. Inzwischen können Stents nach Maß gefertigt werden um auch komplexe Stenosen zu erweitern. Dieser Bifurkationsstent wurde nach Maßen und Winkeln aus einer virtuellen Bronchoskopie hergestellt noldrahtstents mit nahezu beliebigen Dimensionen und Formen herstellen können. - Abb. 15.27 zeigt einen maßgefertigten Nanjing-Y-Stent für eine Bifurkationsstenose. Die Maße wurden aus einer virtuellen Bronchoskopie abgeleitet. Nach einer Skizze wurde der Stent innerhalb weniger Tage in Asien hergestellt (Vertrieb Leufen).

Es gibt wenig Studien über Therapieversager und Nebenwirkungen, aber allgemein kann man sagen, dass Komplikationen von Kunststoffstents leichter zu beherrschen sind als die von Metallstents, da Metallstents in die Schleimhaut einwachsen und nicht mehr ohne erhebliche Verletzungen repositioniert bzw. entfernt werden können.

Für die Tumortherapie kommen nur Silikonstents oder ummantelte Metallgitterstents infrage. Durch eine rechtzeitige Stenteinlage kann eine sofortige Linderung der Luftnot erreicht werden. Auch Patienten im Endstadium einer Tumorerkrankung kann man durch eine Stenteinlage die Qual des Erstickungstods nehmen. Alle anderen Behandlungsoptionen mit tumorverkleinernder Wirkung bleiben auch nach palliativer Stenteinlage bestehen. So kann man zunächst einen Tumor mit dem Laser verkleinern, abschließend einen Stent legen und dann perkutan oder endobronchial bestrahlen oder chemotherapieren. Da durch derartige Maßnahmen eine Tumorverkleinerung erzielt werden kann, sind auch bei Tumorpatienten Stents zu bevorzugen, die man wieder entfernen kann.

Andere Indikationen für tracheobronchiale Platzhalter sind radiogene Strikturen, Anastomosenstrikturen und Abknickungen. In Einzelfällen kann man Stents auch zum Abdichten von Fisteln (• Abb. 15.28), Dehiszenzen und Stumpfinsuffizienzen einsetzen. Nur wenn alle anderen Maßnahmen ausgeschöpft sind, sollten Stents zur Behandlung von Malazien eingesetzt werden. Tracheobronchiale Kollapszustände können zwar mit Stents versorgt werden, die Komplikationsraten sind aber hoch und nur wenige Patienten profitieren wirklich von Stenteinlagen. Umstritten ist die Indikation besonders bei benignen Stenosen. Noch ist die Tracheateilresektion Therapie der
- Abb. 15.28. Stumpffistel nach Pneumonektomie. Ein einseitig mit Silikon verschlossener Bifurkationsstent wird zum Abdichten eingesetzt
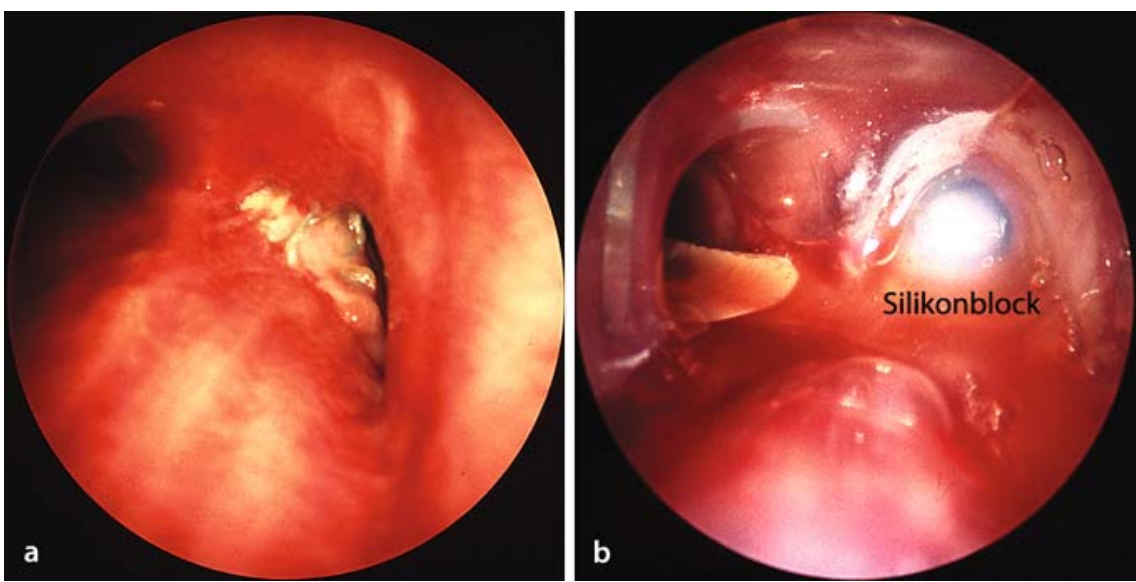


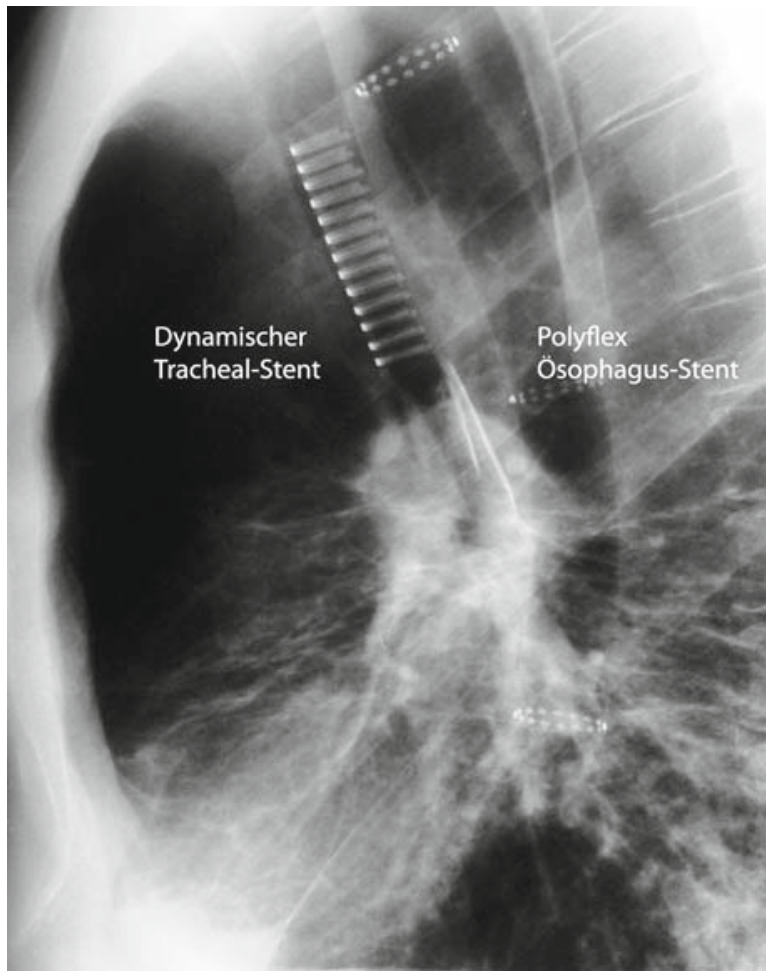

- Abb. 15.29. Doppelstenting zum Abdichten einer großen ösophagotrachealen Fistel. In gleicher Sitzung können ein Ösophagusstent, ein Trachealstent und eine PEG-Sonde eingelegt werden

Wahl, aber bei hohem Operationsrisiko werden Stenteinlagen zunehmend als Alternative angesehen. Es wird sich zeigen, ob die neueren Stents weniger Langzeitprobleme bereiten und ob damit das Indikationsspektrum ausgeweitet werden kann.

\section{Kurative Intention}

Auch bei kleinen Tumoren bleibt die Operation der Goldstandard, aber bei funktionell inoperablen oder nur mit höchstem Risiko operablen Patienten mit kleinen zentralen Tumoren kann mit endoskopischen Verfahren eine Tumorvernichtung versucht werden. Mit Hochdosisbrachytherapie bis $35 \mathrm{~Gy}$ wurden histologisch gesicherte komplette lokale Tumorkontrollraten von $83 \%$ erzielt.

Bei multilokulären kleineren ( $<1 \mathrm{~cm}$ Durchmesser) sowie flach spreitenden Tumoren kann man mit der PDT mit Photofrin eine langfristige Tumoreradikation in bis zu $80 \%$ erreichen. Liegen größere Tumorvolumen vor, erscheint die Kombination von PDT und anschließender Brachytherapie überlegen. Diese aufwendigste Kombination sollte $\mathrm{Pa}$ tienten ohne Metastasierung vorbehalten bleiben. Auch mit dem Elektrokauter und mit der Kryotherapie lassen sich mikroinvasive Tumore in über $80 \%$ der Fälle beseitigen, sofern diese Verfahren lokaltechnisch durchführbar sind.
Voraussetzung ist bei allen genannten endoskopischen Verfahren, dass der Tumor die Bronchuswand nicht durchbrochen hat. Fluoreszenzdiagnostische Verfahren und endobronchialer Ultraschall sind hilfreich, um diese Patienten zu identifizieren.

\section{Zukunftsperspektiven}

An bronchoskopisch entnommenen Biopsieproben kann man genetische Marker bestimmen und Chemosensitivitätsuntersuchungen durchführen, um onkologische Therapien zu optimieren. Zukünftige Entwicklungen der Bronchologie werden sicher immunologische und molekulare Lokaltherapien beispielsweise mit Antisenseoligonukleotiden sowie Gentherapien einschließen.

In zentrale Tumore kann man schon jetzt über das Bronchoskop Tumorsuppressorgene transfizieren. Es wäre denkbar, bei Heilungsstörungen wie Dehiszenzen lokal Wachstums- oder Proangiogenesefaktoren zu injizieren und andererseits bei Tumorausbreitungen Antiangiogenesefaktoren zu geben.

Die Möglichkeiten der photodynamischen Therapie sind sicher noch nicht ausgeschöpft. Eine bessere prothetische Versorgung mit individuell angefertigten, medikamentenbeladenen oder bioresorbierbaren Stents wird das Indikationsspektrum erweitern.

Nicht nur in der Tumortherapie hat sich die interventionelle Bronchologie etabliert. In den letzten Jahren werden auch bei anderen pneumologischen Erkrankungen endoskopische Verfahren eingesetzt. Mittels bronchoskopischer Hochfrequenzablation hat man glatte Muskelzellen verödet, um Patienten mit schwerstem Asthma zu helfen. Die ersten Langzeitergebnisse sind ermutigend. Zur Entblähung bullöser Areale werden Bypass-Stents durch die Bronchuswand in die Bullae eingelegt. Hiervon können Patienten mit Emphysemen profitieren. Als Alternative zur chirurgischen Lungenvolumenreduktion werden verschiedenste Ventile oder Stopfen in Segmentbronchien eingelegt (• Abb. 15.31). Durch Umverteilung der Atemluft und Induktion einer Atelektase erreicht man eine Verbesserung der Lungenfunktion. Ohne Operation können durch diese bronchoskopische Maßnahme viele Emphysempatienten wieder belastbarer werden.

Die interventionelle Bronchologie wird ihren festen Platz in der Pneumologie behalten und ausbauen.

\subsubsection{Thorakoskopie}

H.C. Jacobaeus, ein Internist aus Stockholm, veröffentlichte 1910 die ersten Erfahrungen über den Einsatz von Zystoskopen in serösen Höhlen. Er führte zunächst diagnostische Untersuchungen der Bauch- und Pleurahöhle durch, beschrieb primäre und sekundäre Tumore von 

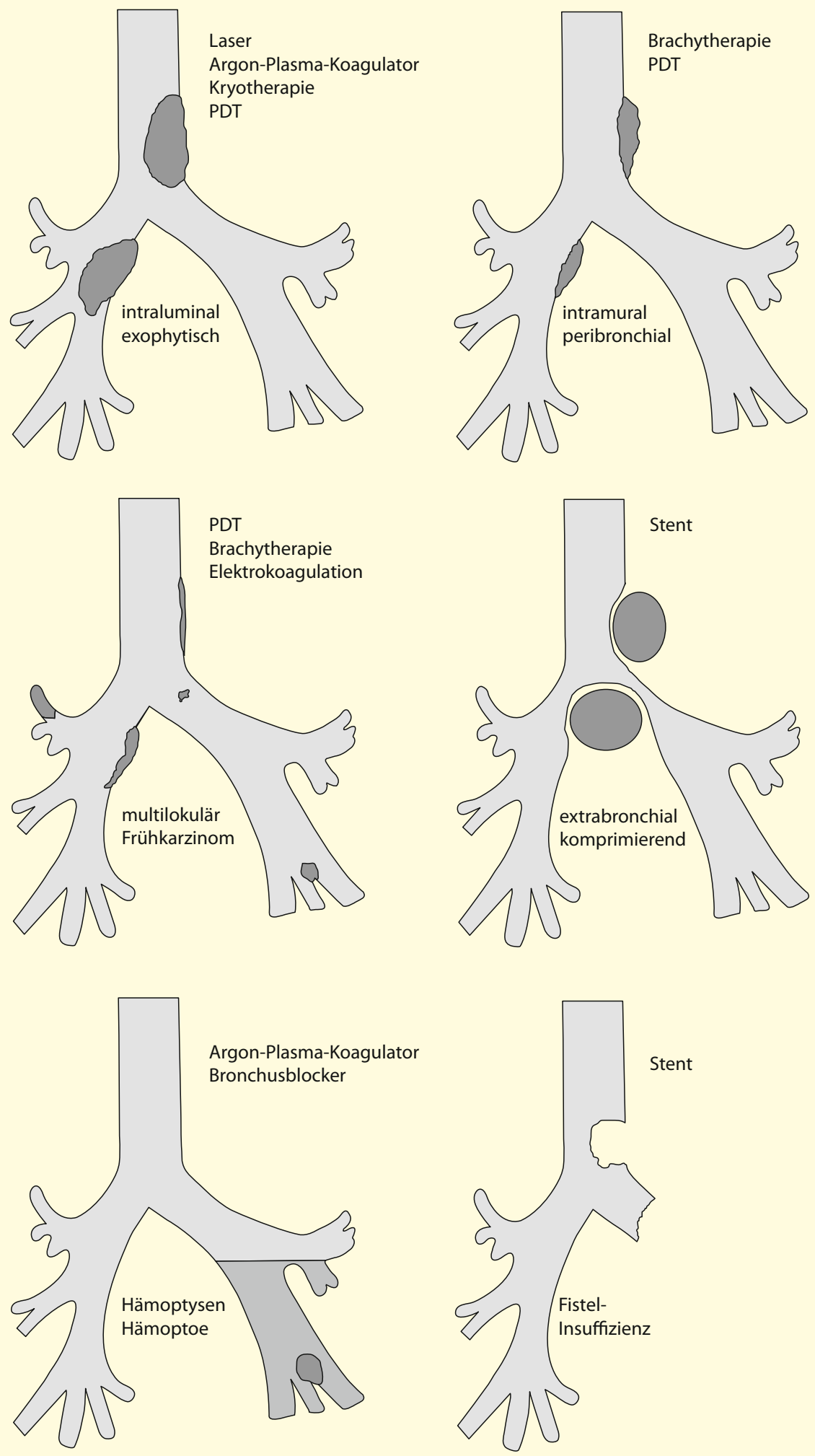

- Abb. 15.30. Schema der Indikationen für bronchoskopische Interventionen: Intraluminales, exophytisches Wachstum links oben, intramurale Tumorausbreitung rechts oben, multilokuläre Frühtumore links

Mitte, externe Kompression rechts Mitte, endobronchiale Blutung links unten, Fistel, Dehiszenz rechts unten 


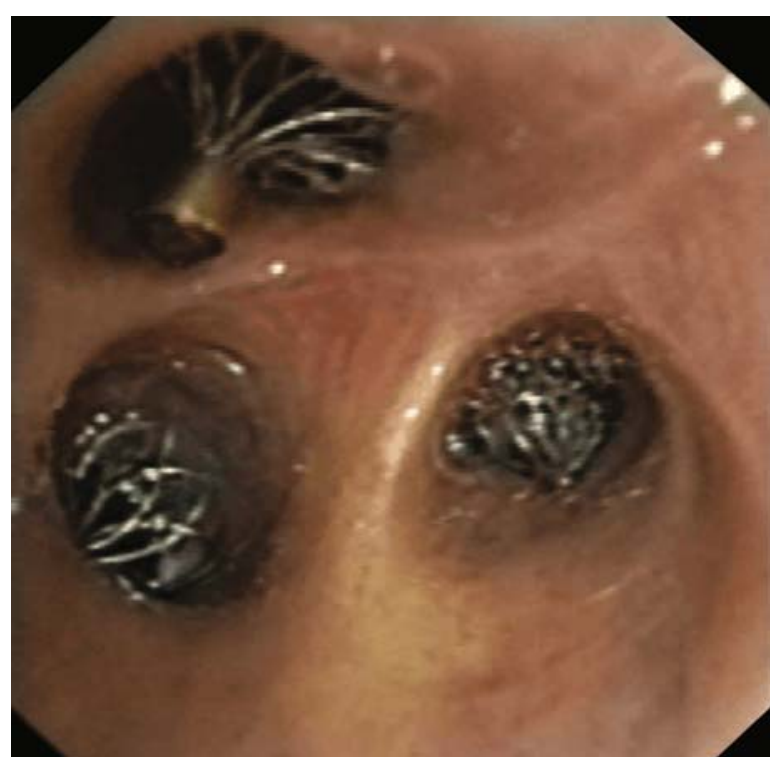

- Abb. 15.31. Zur endobronchialen Emphysembehandlung werden Verschlussstopfen, Ventile oder Bypass-Stents eingesetzt. 4 Monate nach bronchoskopischer Einlage der 3 Occlusalstents in die Oberlappensegmentbronchien ist der Patient deutlich belastbarer

parietaler und viszeraler Pleura, Lunge und Mediastinum. Bis 1925 behandelte er mit der Technik, die heute medizinische Thorakoskopie genannt wird, über 100 spezifische und unspezifische Empyeme. Schnelle Verbreitung fand die thorakoskopische Pneumolyse mit dem Elektrokauter, um einen therapeutischen Pneumothorax anlegen zu können. Dieser Eingriff wurde weltweit als JacobaeusOperation bekannt.

Mit den besseren medikamentösen Behandlungsmöglichkeiten der Tuberkulose änderte sich der Schwerpunkt der Thorakoskopie hin zu den diagnostischen Eingriffen. Durch die Entwicklungen der videoassistierten, minimal invasiven Chirurgie haben sich die Indikationen der internistischen Thorakoskopie weiter verändert. Es gibt immer wieder hitzig geführte Debatten, ob ein spezieller Eingriff von Internisten oder Thoraxchirurgen durchgeführt werden sollte. Meine persönliche Meinung ist, dass man demjenigen den Eingriff überlassen sollte, der ihn sicher beherrscht und der ihn schnell, kostengünstig und effizient durchführen kann (also dem Pneumologen).

\section{Indikationen}

Die häufigsten und allgemein akzeptierten Indikationen für die Thorakoskopie sind:

- der Pleuraerguss, maligne, entzündlich, spezifisch,

- die Pleurakarzinose,

- das Pleuramesotheliom,

- das Pleuraempyem,

- der Spontanpneumothorax.
Die häufigsten therapeutischen Maßnahmen sind Spülungen und Pleurodesen.

\section{Instrumentarium und Durchführung}

Während die minimal invasive videoassistierte Thoraxchirurgie in Allgemeinnarkose mit einem Doppellumentubus durchgeführt wird, erfolgt die internistische Thorakoskopie in Lokalanästhesie am sedierten, spontanatmenden Patienten. Natürlich müssen die Kontraindikationen, insbesondere Gasaustauschstörungen, kardiale Einschränkungen und Gerinnungsstörungen (INR $>2$ ) ausgeschlossen werden. Die üblichen Versorgungsmaßnahmen wie Venenzugang, Anlage von Pulsoximeter, EKG-Monitor und Sauerstoffkatheter werden getroffen, und der Patient wird auf die Seite gelagert. Die Punktionsstelle wird sonographisch oder mit Durchleuchtung in dieser Körperlage markiert.

Nach Sedierung und großzügiger Lokalanästhesie (15-30 ml Lidocain 1\%) geht man meistens in der mittleren Axillarlinie ein. Bei einem Pneumothorax wird allgemein der 3. oder 4., bei einem Erguss der 5. oder 6. Interkostalraum gewählt. Die diagnostische Thorakoskopie erfordert häufig die Anlage eines Pneumothorax. Um diesen kontrolliert zu induzieren, wird vorteilhafterweise ein sogenannter Pneumothoraxapparat mit Manometer verwendet. Bei größeren Ergüssen oder einem bereits bestehenden Pneu ist dies nicht notwendig.

Unter Beachtung der bekannten Gefahren (Interkostalarterien) geht man schließlich mit einem Trokar ein. Üblich sind Durchmesser zwischen 7 und $10 \mathrm{~mm}$, selten werden 12-mm-Hülsen verwendet. Die semirigiden Trokarhülsen geben dabei etwas mehr Spielraum für die starren Instrumente. Seit einiger Zeit sind semirigide (bzw. semiflexible) Thorakoskopieoptiken verfügbar. Selten ist es notwendig mit einem zweiten Zugang zu arbeiten.

Die diagnostische Ausbeute ist bei der Thorakoskopie mit etwa 90\% wesentlich höher als bei Punktionen und alleinigen Ergussuntersuchungen. Nach ausgiebiger Inspektion und Probeentnahme für feingewebliche, biochemische und mikrobiologische Untersuchungen können therapeutische Maßnahmen wie das Beseitigen von Kammerungen ergriffen werden. Wenige Internisten führen Abtragungen und Kauterisierungen von Blasen bei Pneumothoraces durch. Die am weitesten verbreitete therapeutische Prozedur ist sicher in den meisten Zentren die Talkumpleurodese.

\section{Pneumolyse und Pleurodese}

Eine Pleurodese bei malignen Ergüssen ist nur dann Erfolg versprechend, wenn die viszerale und die parietale Pleura großflächig in Kontakt gebracht werden können. Um Verwachsungsstränge, Fibrinsegel oder Kammern 


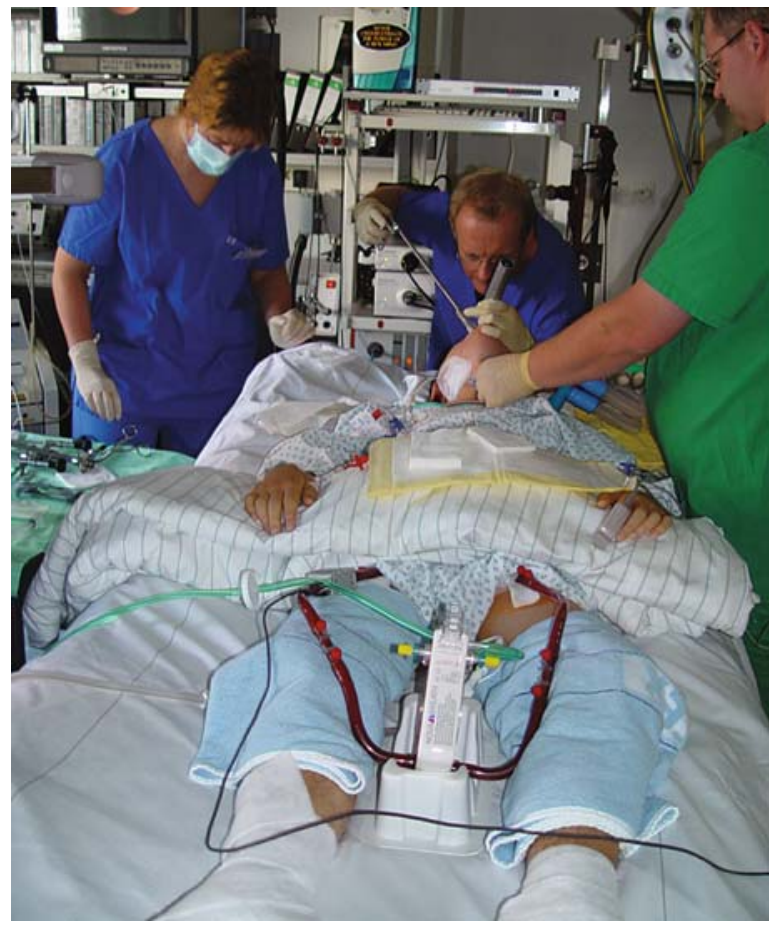

- Abb. 15.32. Manche Eingriffe sind nur durch maximalen Einsatz mit großer Kooperation möglich. Bei einem Patienten mit Trachealverbrennung und respiratorischer Insuffizienz erfolgt die Stenteinlage unter extrakorporaler $\mathrm{CO}_{2}$-Elimination (ECLA)

ausreichend beseitigen zu können und die Expansionsfähigkeit der Lunge für eine Erfolg versprechende Pleurodese zu erreichen, müssen manchmal 2 oder 3 Trokare eingeführt werden. Bei Pleuramesotheliomen muss man sich der Gefahr bewusst sein, möglicherweise Tumorzellen in den Stichkanal zu bringen. Man sollte sich daher auf das Notwendigste beschränken und evtl. Nachbestrahlungen (extern oder Brachytherapie) des Stichkanals durchführen. Sofern man Adhäsionen löst, müssen wenigstens ein Elektrokauter und isolierte Instrumente zur Verfügung stehen. Schwere Blutungen sind absolute Raritäten. Der Endoskopiker muss aber in jeder Hinsicht darauf vorbereitet sein und muss sicherstellen, dass notfalls eine thoraxchirurgische Intervention erfolgen kann.

Bei sehr lokalisiertem Tumorbefall der parietalen Pleura kommt auch eine photodynamische Therapie infrage. Hierzu muss man aber Photosensitizer mit hoher Quanteneffizienz benutzen, da die Bestrahlungszeiten sonst bei der großen Fläche untragbar hoch wären (mehrere Stunden). Bei Verwendung von m-THPC (Foscan) haben wir bei kleinen Tumoren eine Eradikation erreichen können. Derartige Therapien sind aber rein experimentell und sollten nur als Ultima ratio erwogen werden. Auch die lokale Applikation von Chemotherapeutika,
$\beta$-Interferon oder Bioadhäsiven wie Fibrinkleber ist Einzelfällen vorbehalten.

Bei den Pleurodeseverfahren hat die thorakoskopische Talkumpoudrage die höchsten Erfolgsaussichten und wird weltweit als Standard akzeptiert. Häufigste Indikation hierfür ist der maligne Pleuraerguss, seltener wird die Talkumpoudrage zur Behandlung des Pneumothorax durchgeführt. Verwendet wird steriles, faserfreies (asbestfreies) Talkumpuder. Wir pudern meistens mit $5 \mathrm{~g}$, die Obergrenze liegt bei $10 \mathrm{~g}$ Puder. Um das Talkum aufzubringen, wird ein Sprühvernebler mit Pumpballon verwendet. Während des Sprühens wird der Katheterstab in alle Richtungen gedreht, um eine möglichst gleichmäßige Bestäubung zu erreichen.

Sprühkatheter mit Optik sind entwickelt worden. Alternativ kann ein vorgefertigtes Talkumspray mit Treibmitteldose und Kanülen verwendet werden (Steritalc, Novatech). In jedem Fall muss man sicherstellen, dass "geeignetes" Talkum verwendet wird. Es muss asbestfrei sein und sollte optimale Partikelgrößen haben. Nachdem man sich durch abschließende Inspektion überzeugt hat, dass alle Pleuraund Zwerchfellareale von dem Puder erreicht wurden, wird mindestens eine großlumige Drainage eingelegt und über ein Wasserschloss mit dem Sog verbunden.

Die Erfolgsraten einer korrekt ausgeführten Talkumpoudrage liegen bei über $90 \%$. Bei Rezidiven kann eine Flüssigtalkumpleurodese (Slurry) oder eine Pleurodese mit Tetrazyklin versucht werden. Da den Therapieversagern meistens eine nicht ausreichende Expansion der Lunge zugrunde liegt, sollte aber die Möglichkeit der chirurgischen Intervention mit Pleurektomie erwogen werden. Eine Alternative ist die Einlage von PleurexKathetern (Matthys-Drain ${ }^{\circ}$ ). Hiermit kann der Patient falls erforderlich täglich seinen Erguss ablassen, ohne dass neu punktiert werden muss. Trotz nachlaufender Ergüsse kann man damit einem Patienten die Lebensqualität erheblich verbessern.

\section{Ösophagoskopie}

Die starre Ösophagoskopie wird heute nur noch selten praktiziert. Eine Indikation ist das Bergen verschluckter Fremdkörper. Wegen der unmittelbaren anatomischen Nähe zur Trachea muss sich der Pneumologe mit dem Ösophagus beschäftigen, wenn Tumore, Strikturen, Verletzungen oder Fisteln den oberen aerodigestiven Trakt befallen. Bei mediastinalen Prozessen kommt es häufiger zu der Kombination von Schluckstörung und zentraler Atemwegsobstruktion. Die Therapieinstrumente sind ähnlich. Laser, Argonplasmakoagulator und Dilatatoren werden verwendet. Bei der photodynamischen Therapie wählen wir am Ösophagus kürzerwelliges, weniger tief eindringendes Licht (514 $\mathrm{nm}$ bei Photofrin $\left.{ }^{\circledR}\right)$, um Mukosaschäden und Fisteln zu vermeiden. Zur Dilatation von 
Ösophagusstrikturen verwenden wir meistens SavaryGillard-Bougies, seltener auch Dilatationsballons. Wann immer ein Ösophagusstent eingelegt wird, muss man sich davon überzeugen, dass nicht die Trachea oder die Bifurkationsregion komprimiert wird. Eine gleichzeitige Bronchoskopie ist obligatorisch.

Ösophagotracheale Fisteln können als Folge einer primären Tumorerkrankung des Ösophagus oder der Trachea auftreten. Sie treten auf bei Tumorrezidiven, aber auch bei R0-Resektionen oder nach Strahlentherapie als Folge von lokalen Versorgungsstörungen des Gewebes. Selten sind iatrogene Schäden (Intubation) oder Drucknekrosen durch den Cuff eines Tubus oder einer Trachealkanüle. Diese Fälle sollten chirurgisch und nicht endoskopisch versorgt werden.

Das klinische Leitsymptom einer großen Fistel sind quälende Hustenattacken bei Nahrungsaufnahme. Fisteln können endoskopisch behandelt werden mit Fibrinverklebungen, der Einlage von Ösophagusprothesen, Trachealstents oder beidem. Die besten Ergebnisse bezüglich Überlebenszeit und Gewinn an Lebensqualität haben wir mit der gleichzeitigen Implantation eines Ösophagusstents und eines Trachealstents ( $\bullet$ Abb. 15.29). Um auch bei inkompletter Abdichtung eine ausreichende Ernährung des Patienten zu sichern, lege ich in der gleichen Sitzung (in Allgemeinnarkose) eine PEG-Sonde. Eine sehr kritische Region, für die es bisher noch keine überzeugende Therapie gibt, ist das obere Ösophagusdrittel und die kehlkopfnahe obere Trachea.

\section{Literatur}

Beamis JF, Mathur PN, Mehta A (Hrsg.) (2004) Interventional Pulmonary Medicine. Dekker, New York

Bolliger CT, Mathur PN (Hrsg.) (2000) Interventional Bronchoscopy. Karger, Basel

Bolliger CT, Sutedja TG, Strausz J, Freitag L (2006) Therapeutic bronchoscopy with immediate effect: laser, electrocautery, argon plasma coagulation and stents. Europ Respir J 27(6):1258-71

Deutsche Gesellschaft für Pneumologie (1998) Empfehlungen zur bronchoskopischen Behandlung tracheobronchialer Verschlüsse, Stenosen und muraler maligner Tumoren. Pneumologie 52:243248

Dobbertin I, Dierkesmann R (Hrsg.) (2004) Lehrbuch und Atlas der Bronchoskopie. Huber, Bern

Freitag L, Macha HN, Loddenkemper R (2001) Interventional bronchoscopic procedures. In: Spiro SG (ed.) Lung Cancer. European Respiratory Monograph 6(17):272-304

Freitag L, Macha NH (2004) Hämoptysen. Internist 45(5):555-64

Freitag L (2006) Bronchoskopie auf der Intensivstation. In: Zerkowski HR, Baumann G (Hrsg.) Herzakutmedizin. Steinkopff, Darmstadt, 2006

Kollofrath O (1897) Entfernung eines Knochenstücks aus dem rechten Bronchus auf natürlichem Wege und unter Anwendung der directen Laryngoscopie. MMW 38:1038-1039

Prakash UBS (Hrsg.) (1994) Bronchoscopy. Raven Press, New York

Rodriguez-Panadero F, Janssen JP, Astoul P (2006) Thoracoscopy: general overview and place in the diagnosis and management of pleural effusion. Eur Respi J 28:409-421

\subsection{Atemkrankheiten und Schwangerschaft}

H. Matthys

\subsubsection{Bedeutung von Atemkrankheiten in der Schwangerschaft}

Die häufigste Klage schwangerer Frauen ist Atemnot. Die Schwangerschaftshyperventilation, Hyperzirkulation und die Gewichtszunahme bis zur mechanischen Behinderung der Ventilation durch den hochstehenden Uterus sind hierfür physiologische Ursachen ( $\triangleright$ Kap. 14.3.5). Zur Tabakentwöhnung bei Schwangeren siehe auch $\triangleright$ Kap. 15.8.7.

\subsubsection{Asthma bronchiale}

Verschiedene Studien berichten, dass ein Drittel der Patientinnen weniger, ein Drittel gleich viel und ein Drittel mehr Asthmabeschwerden in der Schwangerschaft hat als zuvor. Bezüglich des Kindes zeigt eine prospektive Studie keine Nachteile für das Neugeborene (Apgar-Index, neonatales Geburtsalter oder neonatale Symptome), sofern die Frauen gut therapiert werden ( $\triangleright$ Kap. 7.1.11).

(? Bei schlecht therapiertem Asthma vor und während der Schwangerschaft steigt das Risiko für eine Asphyxie bei Kind und Mutter. Die Schwangerschaft ist für Steroide und andere Antiasthmatika keine Kontraindikation.

\subsubsection{Pneumonien}

In der peripartalen Phase sind Pneumonien die häufigste Todesursache. Ein typisches Erregerspektrum gibt es nicht, die Aspirationspneumonie sollte aber stets differenzialdiagnostisch erwogen werden.

Im 3. Trimenon ist die Varizellenpneumonie relativ häufig als Folge der schwangerschafsbedingten zellulären Immunabwehrschwäche (• Abb. 15.33). Um das Mortalitätsrisiko für Mutter und Kind zu reduzieren, sollten Frauen rechtzeitig vor der ersten Schangerschaft gegen alle viralen Pneumonieerreger (HSV, CMV, Influenza etc.) soweit möglich geimpft werden, da eine medikamentöse antivirale Therapie, z. B. mit Aciclovir, Ganciclovir, Interferon alpha, Ribavirin, in der Schwangerschaft ein weit größeres Risiko darstellt. Am häufigsten sind aber auch hier als Pneumonieerreger während der Schwangerschaft und im Kindsbett (Cave: Aspirationspneumonie) Streptococcus pneumoniae, Haemophilus influenzae und sog. »atypische« Keime zu nennen ( $\triangleright$ Kap. 8.3.1). 
- Abb. 15.33. Varizellenpneumonie bei einer 29-jährigen Frau. Restitutio ad integrum nach Beatmung und antibiotischer Abschirmung. Die typischen Hauteffloreszenzen (a), waren zusammen mit der milchglasartigen Trübung im Röntgenbild (b) für die Diagnose ausschlaggebend
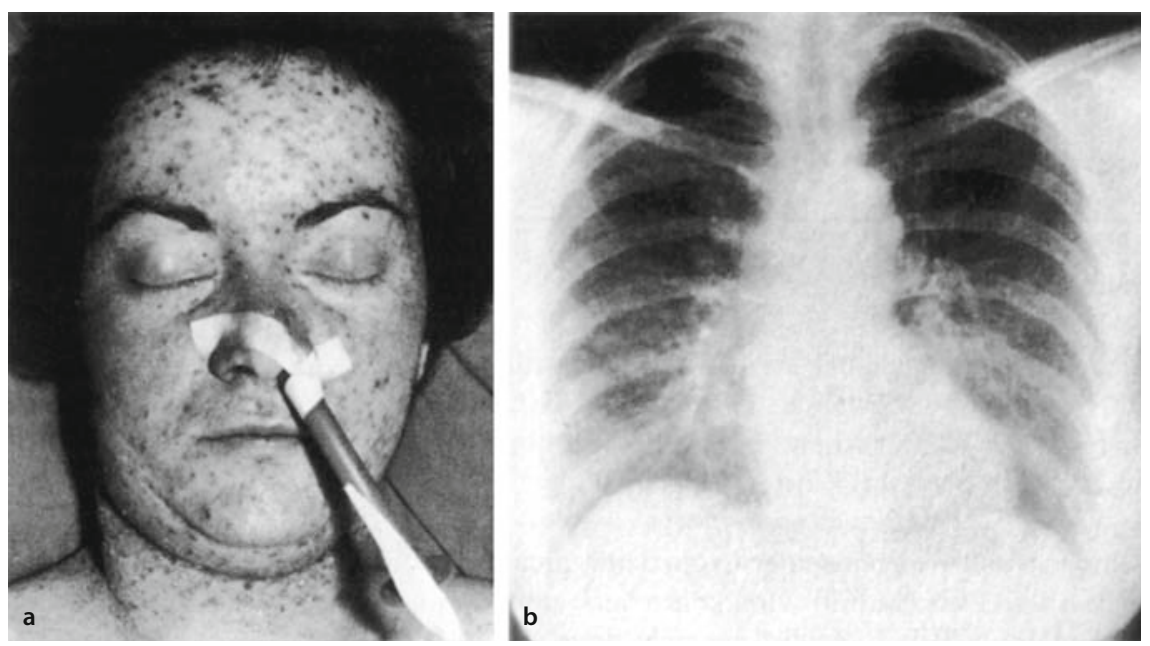

\subsubsection{Lungentuberkulose}

D Kongenital und neonatal erworbene Tuberkulosen haben eine hohe Mortalität (cave: Meningitis). Die Therapie der mütterlichen Tuberkulose verhindert dieses Risiko, ohne das Kind zu schädigen.

Streptomycin sollte wegen seiner Ototoxizität nicht gegeben werden. Pyrazinamid wird kontrovers empfohlen, besonders in den USA wird es nicht angewendet. Isoniacid, Rifampicin und Ethambutol gelten in gewichtsadaptierten Dosen als Standardtherapie, wobei die Substitution von Vitamin B (Pyridoxin) wie bei Nichtschwangeren zur Isoniazidtherapie dazugehört ( $>$ Kap. 8.4.1).

\subsubsection{Pulmonale Hypertonie}

Die pulmonale Hypertonie in Ruhe (sog. manifeste pulmonale Hypertonie) ist eine Kontraindikation für Schwangerschaft und eine Kontrazeptivaindikation, da sie mit einer Mortalität bis 50\% einhergehend ( $\triangleright$ Kap. 9.2).

\subsubsection{Lungenembolien}

Eine Lungenembolie ist die häufigste Ursache prä- und postpartaler plötzlicher Todesfälle. Venostase und Hyperkoagulabilität bei verminderter Fibrinolyse fördern die Thrombosenbildung. Insbesondere ist die Schwangerschaft ein höheres Thromboserisiko als die Kontrazeptivaeinnahme ohne zu Rauchen. Die Duplexsonographie der unteren Extremitäten ist der sensitivste Test, die Ausgangsthrombose $\mathrm{zu}$ finden. Ventilations-PerfusionsSzintigramme sind bei Verdacht auf Lungenembolie in- diziert. Sie sind weniger strahlenbelastend und weniger invasiv als das Spiral-CT. Antikoagulierung mit Heparin ist in allen positiven Fällen indiziert, da niedermolekulares Heparin nicht durch die Plazenta geht und den Fetus daher nicht beeinträchtigt ( $\$$ Kap. 9.3).

\subsubsection{Mukoviszidose}

Im Gegensatz zu Männern haben Frauen mit Mukoviszidose eine normale Fertilität, außer in fortgeschrittenen Fällen mit respiratorischer Insuffizienz und Unterernährung ( $>$ Kap. 7.3 ).

\subsubsection{Lungenfibrosen}

Frauen mit familiären, idiopathischen und anderen erworbenen Lungenfibrosen (Pneumokoniosen, exogen-allergische Alveolitiden) sowie im Rahmen von Systemerkrankungen haben kein erhöhtes Mortalitätsrisiko als Folge der Schwangerschaft, sofern keine wesentliche respiratorische Insuffizienz und pulmonale Hypertonie besteht. Insbesondere wird der Verlauf einer Sarkoidose durch die Schwangerschaft nicht entscheidend beeinflusst.

\subsubsection{Schlafapnoesyndrom}

Schnarchen kommt bei schwangeren häufiger vor als bei nicht schwangeren Frauen. Schnarchen ist aber kein nachgewiesener Risikofaktor für das Kind und die Mutter. Bei Symptomen der Mutter wie exzessive Tagesmüdigkeit etc. sollte eine polysomnographische Exploration erfolgen und evtl. eine CPAP-Therapie eingeleitet werden. Dies gilt 
insbesondere für die Präeklampsie, die sich durch CPAPAtmung bessert ( $\triangleright$ Kap. 14.3.5).

\subsubsection{Fliegen und Tauchen}

Pilotinnen und in der Schwangerschaft aktive Taucherinnen haben eine erhöhte kindliche Missbildungsrate. Es sollte daher insbesondere vom Gerätetauchen in der Schwangerschaft abgeraten werden. Für ein erhöhtes Dekompressionsunfallrisiko von Frauen gegenüber Männern gibt es keine Evidenz. Allerdings scheint zu Beginn der Menstruation eine erhöhte Dekompressionsunfallgefahr zu bestehen, wahrscheinlich als Folge einer gestagenbedingten vermehrten Flüssigkeitseinlagerung in den Körpergeweben. Mit dem Tauchsport sollte erst 2-3 Monate nach der Geburt wieder begonnen werden.

Brustimplantate und andere Gasblasen enthaltende Strukturen (Zahnfüllungen) und Prothesen können ein erhöhtes Risiko für Dekompressionsunfälle darstellen (s. auch $>$ Kap. 5).

\section{Literatur}

ATS (1994) Treatment of tuberculosis and tuberculosis infection in adults and children. Am J Respir Crit Care Med 149:1359-1374

Demers C, Grissberg JS (1992) Deep venous thrombosis and pulmonary embolism in pregnancy. Clin Chest Med 13:645-656

Hanania NA, Cazzola M (2006) Acute espiratory failure in Pregnancy. In: Narva S, Welte T (eds.) Respiratory emergencies. Eur Respir Mon 36:241-258

King TE Jr (1992) Restrictive lung disease in pregnancy. Clin Chest Med 13:607-622

Koboloff RM, Fitz Simmons SC, Fiel SB (1992) Fertility and pregnancy in patients with cystic fibrosis. Clin Chest Med 13:607-622

Laube DI, Poceta JS, Morales MC, Peacocr MD, Mitler MM (1996) Self reported snoring in pregnancy. Association with fetal outcome. Chest 109:885-889

Mapp C, Buist S (eds.) (2003) Respiratory diseases in women. Eur Respir Mon 25

Minerbi-Codish I, Fraser D, Avnun L, Glezerman M, Heimer D (1998) Influence of Asthma in Pregnancy on Labor and the Newborn. Respiration 65:130-135

Neumann SM, Freyschmidt J, Holland BR, Henschel M, Gahnem NR (1997) Vergleich der Perfusions-Ventilations-Szintigraphie mit dem Spiral-CT bei akuter Lungenembolie. Med Klin 92:635-641

Olesen C, Thrane N,Soerensen HAT, Olsen J (2001) A population-based study of asthma drugs during pregnancy: changing the intensity of asthma therapy and perinatal outcomes. Respiration 68:256-261

Jeldio SM (1992) Dyspnoea during pregnancy distinguising cardiac from pulmonary causes. Clin Chest Med 13:567-585

Rigk N, Kalassian KG, Gilligan T, Drazin MI, Daniel DL (1996) Obstetric complications in pulmonary and critical care medicine. Chest 110:791-809

Rodriguez I, Niedermann MS (1992) Pneumonie complicating pregnancy. Clin Chest Med 13:679-691

Semedstad KG, Cramb R, Morrison DH (1994) Pulmonary hypertension and pregnancy, a series of 8 cases. Can J Anaesth 41:502-512

Stenius-Arniala B, Pürila P, Teramo K (1988) Asthma and pregnancy a prospective study of 198 pregnancies. Thorax 43:12-18

Wurm K (Hrsg) (1997) Sarkoidose. Thieme, Stuttgart

\subsection{Tabakentwöhnung}

P.L. Bölcskei

\subsubsection{Möglichkeiten der Tabakkontrolle}

Die Erhöhung des Zigarettenpreises durch Anhebung der Tabaksteuer ist einer der effektivsten Wege zur Reduktion des Tabakkonsums. Die Tabaksteuererhöhung bringt zweifachen Vorteil mit sich: Neben der Reduktion des Tabakkonsums schafft diese Maßnahme eine finanzielle Unterstützung für weiterführende Tabakkontrollprogramme. Voraussetzung ist allerdings der zweckgebundene Einsatz dieser Mittel, z. B. für die Finanzierung von Raucherberatungs- und Tabakentwöhnungsmaßnahmen. Insbesondere für Jugendliche und Personen mit geringem Einkommen gilt, dass die Erhöhung der Tabaksteuer um 10\% zu einer um 13\% geringeren Raucherprävalenz führt.

Weitere gesetzgeberische Maßnahmen bezwecken vor allem den Schutz der Nichtraucher vor dem Passivrauchen. Der Nichtraucherschutz dient zugleich der Gesundheitsförderung der Aktivraucher: Unter anderem lernen sie, auf das Gros der automatisch gerauchten Zigaretten zu verzichten. Weitere gesetzgeberische Maßnahmen sind die Einschränkung der Werbung der Tabakindustrie und die Erschwerung des Zugangs für Minderjährige zu Tabakwaren. Wenn Jugendliche ihre Zigaretten nicht mehr aus dem Automaten ziehen können, kann das zu einer Verzögerung des Rauchbeginns beitragen.

\subsubsection{Verhaltens- und soziale Aspekte der Tabakabhängigkeit}

Psychologische Theorien verstehen die Abhängigkeit als erlerntes, pathologisches Verhalten, das durch klassische sowie operante Konditionierungsvorgänge verfestigt wird. Klassische Konditionierungsvorgänge sind für die Automatie des Rauchverhaltens verantwortlich. Die starke Kopplung des Rauchverhaltens an bestimmte Situationen und an mit der Nikotinaufnahme verbundene sensomotorische Reize führen zu einer sekundären Konditionierung. Dem Prinzip der operanten Konditionierung entspricht - neben diversen anderen Verstärkungseffekten - die negative Verstärkung, die durch Nikotinzufuhr und damit durch die Besetzung der freien Rezeptoren erreicht wird: Unangenehme Entzugssymptome werden durch Aufrechterhaltung des Nikotinkonsums vermieden.

Reiz-Antwort-Beziehungen (z. B. der Griff zur Jackentasche bei der Suche nach der Zigarettenpackung) und Konditionierungsmechanismen wie die dauerhafte Verknüpfung zwischen Geruch des Zigarettenrauchs und Rauchen (»habit learning«) stabilisieren den Tabakkonsum bzw. 
führen nach Abstinenz zu Rückfällen. Im übertragenen Sinne kann hiermit der Schlüsselreiz zur Sucht den Motor der Abhängigkeit jederzeit erneut starten. Das ventrale Striatum scheint an diesen Konditionierungsmechanismen beteiligt zu sein. Klinische Bedeutung erlangt hierbei, dass unser Gehirn für Lernprozesse, die Geruchs- und Geschmacksreize betreffen, besonders empfänglich ist. Nikotin und Alkohol sind klassische Beispiele für Suchtstoffe mit einer ausgeprägten sensorischen Komponente.

Wenn Raucher den Rauchstopp vollziehen, so wird es erforderlich, neben der Vorbereitung auf die Symptome des Nikotinentzugs auch für die haptischen Gewohnheiten Alternativen zu finden. Die Gewohnheiten und Konditionierungen sind klinisch auch nach erfolgreicher Tabakentwöhnung schwer korrigierbar. Erste Studien deuten auf eine komplexe Umwelt-Gen-Interaktion mit einem deutlichen erblichen Faktor hin.

Psychosoziale Einflüsse tragen zur Modulierung der Nikotineffekte bei. So können die Erwartung des Nichtrauchens in bestimmten Kulturen oder die Auswirkung von rauchfreien Arbeitsplätzen eine Abstinenz begünstigen. Mit einem Raucher verheiratet zu sein oder einer sozial benachteiligten Gruppe anzugehören, in denen das Rauchen die Norm darstellt, sind unter anderem Faktoren, die das Rauchverhalten begünstigen oder der Rauchfreiheit im Wege stehen.

\subsubsection{Diagnostik der Motivation zum Rauchstopp und der Tabakabhängigkeit}

Die Erfolgschance eines Rauchers, langzeitrauchfrei zu werden, hängt von der individuellen Motivation und dem Grad der Tabakabhängigkeit ab. Die intrinsische Motivation ist eine wichtige Voraussetzung für den Rauchstopp. Die Motivation aufzuhören kann mit der Zeit variieren und wird stark durch die unmittelbare Umgebung beeinflusst.

\section{Ermittlung der Motivationsstärke bezüglich des Rauchstopps}

Die Motivation der Raucher zum Rauchstopp kann am besten mittels einfacher, direkter Fragen geklärt werden. Eine semiquantitative Abschätzung der Motivation erhält der Therapeut durch Befragung der Raucher mithilfe einer 10-stufigen Likert-Skala. Die Motivationsstärke lässt sich anhand eines Eintrags auf der Skala von "sehr stark « bis »überhaupt nicht « ermitteln.

Tabakentwöhnung setzt eine Verhaltensänderung voraus. Dies ist ein dynamischer Prozess, wie er im "transtheoretischen Modell " nach Prochaska und DiClemente beschrieben wird (• Abb.15.34). Der Raucher durchläuft verschiedene Motivationsstadien, bevor er schließlich die Langzeitrauchabstinenz erreicht. Von Stadium zu Stadium nehmen seine Chancen rauchfrei zu werden zu; beginnend beim Stadium des stabilen Rauchers, der noch nicht vorhat, mit dem Rauchen aufzuhören, über das Stadium der "Dissonanz " bis zum Stadium der "Vorbereitung" zum Rauchstopp. Anschließend wird er im Stadium der "Handlung" zum Nichtraucher. Dieses Stadium dauert während der ersten 6 Monate nach dem Rauchstopp an. Dann folgt das Stadium der »Stabilisierung" zum Exraucher. Wie auf der Abbildung zum »Prozess der Tabakentwöhnung nach Prochaska und DiClemente « dargestellt ist, verläuft die Motivationssteigerung nicht streng in Richtung stabilem Exraucher, sondern es treten in den meisten Fällen Rückschritte in vorherige Stadien auf.

Dieses Modell erlaubt eine individuelle Intervention, die in Intensität und Inhalt dem Motivationsstadium des Patienten zum Aufhören angepasst ist.

Dieses Modell trägt dazu bei, die Patienten dort abzuholen, wo sie sich in Bezug auf ihre Motivation befinden. Damit werden Frustration des Therapeuten und das Gefühl der Raucher, missverstanden zu werden, vermieden. Mit der motivierenden Gesprächsführung nach Miller und
- Abb. 15.34. Prozess der Tabakentwöhnung nach Prochaska, DiClemente u. Norcross (1992)

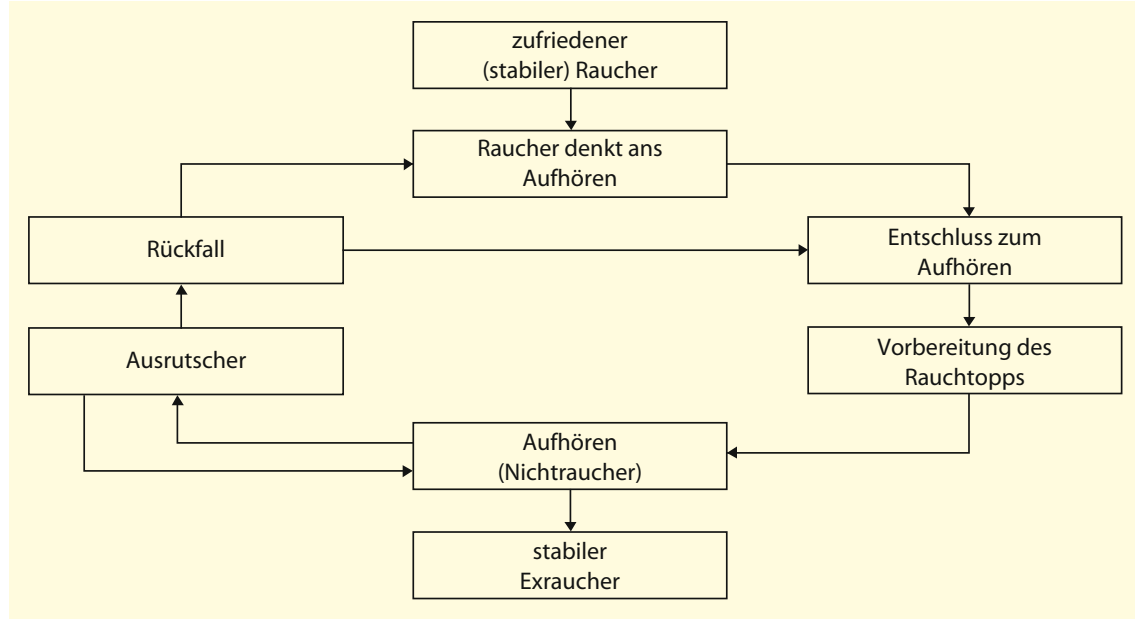


Rollnik können die Patienten mit akzeptablem zeitlichem Aufwand stadiengerecht beraten werden.

Robert West (2006) schlug ein alternatives Modell des Prozesses der Verhaltensänderung (»catastrophic pathways«) vor. Nach diesem Modell befinden sich Raucher in wechselnder Intensität der motivationalen Spannung zum Rauchstopp. Durch Triggerung im Umfeld kann sehr schnell eine Änderung des Motivationsstadiums in Form von »unerwarteten Motivationssprüngen« erfolgen (z. B. in Folge eines Herzinfarkts).

\section{Diagnostisches Erfassen der Tabakabhängigkeit - Nikotin- oder Tabakabhängigkeit?}

Die Beschränkung auf einen Inhaltsstoff des Tabakrauchs - Nikotin - wird der Komplexität der biologischen und psychologischen Faktoren der Abhängigkeit der Raucher nicht gerecht. Daher sollte der Terminus »Tabakabhängigkeit« Anwendung finden.

\section{Diagnostische Kriterien der Tabak- bzw. Nikotinabhängigkeit}

In beiden relevanten diagnostischen Klassifikationssystemen (Internationale Klassifikation von Krankheiten der WHO 1991; Diagnostisches und Statistisches Manual der American Psychiatric Association 1996) findet sich die Diagnose »Tabakabhängigkeit« (ICD-10, F17.2) bzw. »Nikotinabhängigkeit« (DSM IV-305.10). In Deutschland wird nach ICD-10 kodiert.

Im ICD-10 werden 6 Kriterien genannt, von denen 3 in den letzten 12 Monaten gleichzeitig in Erscheinung getreten sein müssen, damit die Diagnose »Tabakabhängigkeit« gestellt werden kann:

- starker Wunsch oder Zwang, Tabak zu konsumieren,

- eingeschränkte Kontrolle über Beginn, Beendigung und Menge des Konsums,

- Entzugserscheinungen bei Reduktion oder Beendigung des Konsums, Konsum, um Entzugssymptome zu mildern,

- Toleranzentwicklung: um eine gleich bleibende Wirkung zu erzielen, sind zunehmend höhere Dosen erforderlich,

- zunehmende Vernachlässigung anderer Aktivitäten und Interessen zugunsten des Konsums,

- anhaltender Konsum trotz des Nachweises von Folgeschäden.

\section{Fagerström-Test für Nikotinabhängigkeit}

Von allen Fragen dieses Fragebogens (Fagerström-Test for Nicotine Dependence, FTND) sind die Anzahl der täglich gerauchten Zigaretten und die Zeit bis zur ersten Zigarette nach dem Aufwachen die wichtigsten Indikatoren der
Abhängigkeit. Deswegen kann in einem kurzen Gespräch mit 2 Fragen der Grad der Abhängigkeit abgeschätzt werden:

- Wann rauchen Sie morgens nach dem Aufwachen die erste Zigarette? Innerhalb von 30 min oder später? Bei starker Abhängigkeit wird die erste Zigarette in der Regel innerhalb von 30 min nach dem Aufwachen geraucht.

- Wie viele Zigaretten rauchen Sie täglich? - Bei mehr als 20 Zigaretten pro Tag wird in der Regel eine starke Abhängigkeit angenommen.

\section{Nachweis von Nikotinmetaboliten und Tabakinhaltsstoffen}

Die Nikotinkonzentration oder die Konzentration seines Hauptmetabolits Kotinin im Blut, Urin oder im Speichel wird in der Forschung häufig als eine objektive Methode zur Messung der Abhängigkeit angewendet. Hierdurch liegt ein zuverlässiges Maß für die Quantität des konsumierten Nikotins als einen Marker für die Abhängigkeit vor. Diese Messung erlaubt die Erfassung des Tabakkonsums der letzten 3-4 Tage.

Die Kohlenmonoxidkonzentration in der Ausatemluft ist ein Maß der aufgenommenen Zigarettenrauchinhalte während der letzten $8 \mathrm{~h}$. Sie erlaubt keine genaue Aussage über die resorbierte Nikotinmenge, die Methode ist jedoch kostengünstig und gibt ein sofortiges Feedback über einen wichtigen toxischen Bestandteil des Zigarettenrauchs im Blut des Rauchers (CO-Hb-Wert). Ein besonderer Vorteil besteht darin, dass der Patient sein Ergebnis unmittelbar am Gerät selbst ablesen kann. Hierdurch wird erkennbar, ob in den letzten $8 \mathrm{~h}$ überhaupt geraucht wurde und ob viel oder wenig.

\subsubsection{Behandlung der Tabakabhängigkeit}

Tabakentwöhnungsinterventionen sind sowohl in den allgemeinärztlichen und fachärztlichen Praxen als auch in den Krankenhäusern in Deutschland, Österreich und der Schweiz bisher unterrepräsentiert, obwohl diese Maßnahmen leicht $\mathrm{zu}$ erlernen und in erheblichem Maße kosteneffektiv sind (Batra et al. 2006, Raupach et al. 2007, ÖGAM 2007, Cornuz et al. 2004). Rauchen verursacht mehr gesundheitliche Schäden als z. B. arterielle Hypertonie oder Hypercholesterinämie, die in hausärztlichem Bereich viel häufiger erkannt und leitliniengerecht therapiert werden. Ein weites Angebot der Tabakentwöhnung kann eindeutig mehr Lebensjahre retten und kosteneffektiver sein, als die meisten einfach anwendbaren Interventionen in der Medizin.

Hört ein Patient bis zum 35. Lebensjahr mit dem Rauchen auf, büßt er an Lebenserwartung nicht wesent- 
lich ein. Raucht er weiter, muss er mit einem Verlust an Lebenserwartung von ca. 7 Jahren rechnen, das entspricht für einen mittelstarken Raucher umgerechnet eine Lebenszeitminderung von $11 \mathrm{~min}$ pro Zigarette. Raucher, die als Jugendliche mit dem regelmäßigen Tabakkonsum beginnen und als Erwachsene weiterrauchen, müssen mit einer Einbuße von bis zu 23 Lebensjahren rechnen. Grundsätzlich ist voraussehbar, dass 50\% der Raucher vorzeitig an einer tabakassoziierten Erkrankung sterben.

Fletcher und Mitarbeiter wiesen bereits 1977 für die Patienten mit COPD nach, dass der endgültige Rauchstopp in jedem Alter die Gesundheit und die zukünftige Lebensqualität verbessert. Tashkin und Mitarbeiter (2001) wiesen in der Lung Health Study I nach, dass lediglich die absolute Rauchabstinenz in der Lage ist, die jährliche Reduktion der 1-sec-Kapazität zu normalisieren.

Wenn langjährige Raucher im mittleren Lebensalter aufhören, reduzieren sie immer noch deutlich das Risiko, an einem Bronchialkarzinom zu erkranken. Die Bronchialkarzinommortalität in der ersten Hälfte des 21. Jahrhunderts wird vielmehr durch die Erfolge der Tabakentwöhnung als durch Reduktion der Zahl der neustartenden jugendlichen Raucher bestimmt. Die Primärprävention gehört, obwohl sie erst mit erheblicher Verzögerung Benefit zeigt, uneingeschränkt zu den Eckpfeilern der Tabakkontrolle.

Die meisten Raucher hören nach der Schlusspunktvorgehensweise von einem auf den anderen Tag mit dem Rauchen auf. Bei diesem Vorgehen ist es unbedingt erforderlich, ein Rauchstoppdatum festzulegen und eine therapeutische Begleitung anzubieten, um einen Rückfall des Rauchers in die frühere Rauchgewohnheit zu verhindern.

Eine allmähliche Reduktion bevorzugten etwa 20\% der Exraucher. Reduziertes Rauchen wird in den letzten Jahren für stark gefährdete Raucher empfohlen, die zu einem Rauchstopp nicht motiviert oder fähig sind, jedoch bereit, ihren täglichen Tabakkonsum um mehr als 50\% zu verringern. Dieses Vorgehen wird mit einer erniedrigten Gefährdung infolge reduzierter Schadstoffinhalation begründet (»harm reduction«). Es ist jedoch bislang noch nicht gesichert, wie viele der Reduzierer irgendwann auch einen Rauchstopp unternehmen. Ebenso gibt es Hinweise darauf, dass die Vorteile des herabgesetzten Tabakkonsums durch »Kompensationsrauchen«, d.h. durch längeres Anhalten der Luft beim Rauchen und mehr und tiefere Züge an einer Zigarette, zum Teil zunichte gemacht werden (Stead u. Lancaster, 2007).

Für den Nullkonsum als Goldstandard sprechen unter anderem die Ergebnisse von Tverdal und Mitarbeitern (2001). Sie belegen anhand einer Kohortenstudie, dass im Vergleich zu unverändert Weiterrauchenden die reduziert Rauchenden keine signifikante Erniedrigung der Mortalitätsrisiken z. B. für ein Lungenkarzinom erwarten können. Es liegen auch Studien über reduziertes Rauchen bei gleichzeitigem Nikotinersatz vor. Nikotinkaugummi ist in Deutschland seit Kurzem zugelassen, um das reduzierte Rauchen zu erleichtern. Eine multizentrische Studie hat für Nikotinersatztherapie mit Kaugummis mit $4 \mathrm{mg}$ Nikotin ermutigende Ergebnisse erbracht. Das reduzierte Rauchen bleibt daher derzeit Ausnahmefällen vorbehalten (Wennike et al. 2003).

\section{Nichtmedikamentöse Therapie}

Die Voraussetzung der Therapie der Tabakabhängigkeit ist die Identifizierung und Dokumentation der Raucher durch Befragung aller Patienten im Rahmen der üblichen Konsultationen. Dies sollte nach internationalen Empfehlungen mindestens einmal jährlich erfolgen. Aufzeichnungen über Rauchstatus sollten, damit sie gut erkennbar sind, unter den Vitalzeichen der Patienten angeführt werden. Es wird zwischen regelmäßigen Rauchern, Exrauchern, Nierauchern und Passivrauchern unterschieden. Wahre Gelegenheitsraucher betragen lediglich etwa 5\% der Aktivraucher.

Die deutsche SNICAS-Studie zeigte, dass den Hausärzten ein Viertel ihrer rauchenden Patienten nicht bekannt waren. Dabei ist es nachgewiesen, dass Ärzte Ihren Patienten doppelt so häufig zum Rauchstopp raten, wenn ihnen deren Raucherstatus bekannt ist.

Um zeitsparend die Raucherberatung durchführen zu können, empfiehlt es sich, diese als wichtige Maßnahme im Team, d. h. in der Praxis gemeinsam mit den Praxisassistentinnen und im Krankenhaus unter Zuhilfenahme des entsprechend geschulten Pflegedienstes, durchzuführen.

Die Spannbreite der nichtmedikamentösen Maßnahmen reicht von einer 3-minütigen Beratung durch den Arzt bis zu Einzel- oder Gruppentherapie mit 6 oder mehr Kontakten und niederschwelligen Anwendungsformen (wie telefonische Raucherberatung, Raucherberatung im Internet u. a. Maßnahmen unterschiedlicher Intensität).

\section{Kurze Raucherberatung}

Die kurze Raucherberatung dauert nur wenige Minuten und umfasst folgende Punkte:

- Identifizierung der Raucher,

- Feststellung des Stadiums der Ausstiegsmotivation (Veränderungsbereitschaft),

- eindeutiger Rat zum Aufhören.

Kurze Raucherberatungsinterventionen können nach den internationalen Leitlinien auch in Praxen mit begrenztem Zeitbudget implementiert werden. Dazu sind in Praxisund Klinikablauf nur wenige Veränderungen notwendig. Auch wenn das Zeitmanagement in Klinik und Praxis den Einsatz von Kurzinterventionen erfordert, ist be- 
kannt, dass intensive Interventionen höhere Erfolgsraten versprechen.

(1) A

Auch wenn derzeit noch viele Raucher intensive Interventionen ablehnen, haben sie dennoch ein Anrecht auf eine Kurzintervention!

Obwohl der Rat des Arztes von den Rauchern hoch eingeschätzt wird, berichtet lediglich jeder zweite Raucher, dass er von seinem Hausarzt auf sein Rauchverhalten angesprochen wurde. Aufgrund mangelnder Kenntnisse in der Raucherberatung erleben Hausärzte das routinemäßige Erteilen des Rates an ihre rauchenden Patienten häufig als frustrierend und ineffektiv.

Es ist aber in Metaanalysen belegt und im Jahre 2000 durch Fiore und Mitarbeiter publiziert, dass ein kurzer Rat ( $\leq 3 \mathrm{~min}$ ) des Arztes genügt, um die Tabakentwöhnungsrate signifikant zu erhöhen. Die Spontanentwöhnungsrate ohne Kontakt liegt bei 10,9\%. Die Abstinenzrate steigt nach einer kurzen Beratung ( $\leq 3 \mathrm{~min}$ ) auf $13,4 \%$. Eine Beratung mit geringer Intensität (3-10 min) lässt eine Abstinenzrate von $16 \%$ und eine Beratung mit höherer Intensität ( $\geq 10 \mathrm{~min}$ ) von $22,1 \%$ erwarten.

\section{Weitere Schritte}

Wird beim Feststellen der Veränderungsbereitschaft deutlich, dass ein Raucher innerhalb der nächsten 2-4 Wochen das Rauchen aufgeben will, sollte ihm entsprechende Unterstützung in der Vorbereitung des Rauchstopps gewährt werden. Nach dem Rauchstopp sind weitere telefonische und/oder persönliche Termine zur stabilisierenden Nachbetreuung erforderlich.

D Bereits eine 4- bis 6-wöchige Nachbetreuung je nach Bedarf mit 1-3 weiteren Kontakten kann die Erfolgsrate verdoppeln.

\section{Die motivierende Gesprächsführung}

Die Grundprinzipien der motivierenden Gesprächsführung nach Miller und Rollnik sollte nicht nur jeder Tabakund Suchttherapeut, sondern auch jeder in der Patientenversorgung Tätige beherrschen. Die Art und Weise, in der man mit jemandem kommuniziert, macht es wahrscheinlicher oder unwahrscheinlicher, ob sich eine Person verändert. Benützt man einen reflektierenden, unterstützenden Stil, kann der Widerstand und das Argumentieren des Patienten gegen eine Veränderung verhindert werden.

Insbesondere bei dissonanten Rauchern bietet die motivierende Gesprächsführung die geeigneten Techniken, um die häufigsten Hindernisse zu überwinden. Die 6 wichtigsten Hindernisse sind:

- Entzugssymptome in der Vergangenheit,

- Versagensängste,

- Befürchtungen bzgl. Gewichtszunahme,

- mangelnde soziale Unterstützung,
- Depression,

- Freude am Rauchen (meistens lediglich Notwendigkeit zur Linderung von Entzugsbeschwerden).

\section{Kognitive Verhaltenstherapie}

Je stärker die Tabakabhängigkeit, desto größer ist in der Regel der Bedarf der Entwöhnungswilligen an verhaltenstherapeutischer Hilfe. Die wichtigsten Säulen bei der Entstehung und Aufrechterhaltung der Tabakabhängigkeit, nämlich das Modelllernen (in Kindheit und Jugend) und die darauf folgende Einstellung zum Rauchen sowie die verhaltens- und lerntheoretischen Prinzipien der klassischen und operanten Konditionierung, sind zugleich Ansatzpunkte für ein gezieltes kognitiv-verhaltenstherapeutisches Vorgehen. Erlerntes Verhalten kann am effektivsten durch den Einsatz und die Verstärkung alternativer Verhaltensweisen verändert werden.

Sinnvolle Verhaltenstherapeutische Methoden sind:

- Selbstbeobachtung,

- Motivationsprüfung und Vorsatzfestigung,

- Zielsetzung und Zielfestigung,

- Selbstanalyse und Selbstkontrolle,

- Selbstwirksamkeitssteigerung,

- Verpflichtungsbereitschaft,

- kognitive Interventionsstrategien,

- Selbstbelohnung,

- Entspannung.

Beispielsweise können erlernte und eingeübte Entspannungsmethoden in der Abstinenzphase zur Beherrschung von "craving « mit Erfolg eingesetzt werden. Eine häufig angewandte Entspannungsmethode ist die progressive Muskelrelaxation nach Jacobson. Diese lässt sich auch unter Bedingungen am Arbeitsplatz, d.h. in so gut wie jeder Situation anwenden.

\section{Akupunktur}

Es gibt laut Cochrane-Gruppe keinen eindeutigen Beweis, dass Akupunktur alleine eine effektive Methode zur Tabakentwöhnung darstellt.

\section{Hypnose}

Aufgrund sehr großer Unterschiede in Häufigkeit und Intensität der Anwendung in der Tabakentwöhnung liegen für die Effektivität bisher keine eindeutigen Belege vor.

\subsubsection{Verschiedene Therapiesettings}

\section{Einzel- und Gruppensetting}

Einzeltherapie ist eine auf gesicherte Weise wirksame, jedoch sowohl für die Patienten als auch für den Therapeuten nicht zeitökonomische und dadurch kostspielige Therapieform. Metaanalysen zeigen, dass die Tabakentwöhnung in 
Gruppenprogrammen eine ebenso wirksame Therapiemaßnahme wie die Einzeltherapie darstellt. Wesentlicher Vorteil ist hier die intratherapeutische soziale Unterstützung durch die Gruppe. Diese ist insbesondere dann bedeutungsvoll, wenn Entwöhnungswilligen sonst keine Helfer zur Verfügung stehen. Nach neueren Untersuchungen ist die Gruppentherapie der Einzelbehandlung überlegen.

\section{Telefonische Raucherberatung}

In jüngerer Zeit findet auch in Deutschland und der Schweiz die telefonische Raucherberatung als niederschwellige und doch gut wirksame therapeutische Maßnahme zunehmende Beachtung. Man unterscheidet proaktive und reaktive Beratung. Die proaktive Beratung wird nach vorheriger Vereinbarung vom Berater initiiert. Die reaktive Beratung erfolgt aufgrund des Anrufs des zu Beratenden. Die telefonische Raucherberatung kann als selbständige therapeutische Modalität nach festgelegter Vorgehensweise erfolgen. Zum anderen kann die telefonische Raucherberatung als ergänzende Maßnahme nach einer vorher durchgeführten Beratung durch den niedergelassenen Arzt oder den Klinikarzt bzw. nach einem Entwöhnungskurs z. B. in einer Reha-Klinik eingesetzt werden.

Die letzte Cochrane-Auswertung durch Stead und Mitarbeiter (2005) ergab signifikante Ergebnisse der telefonischen Raucherberatung in proaktiver Anwendung. Voraussetzung dafür war, dass die proaktiven Anrufe stets vom selben Berater erfolgten und aus mehr als 3 Beratersitzungen bestanden. Die telefonische Raucherberatung und Therapie hat eine wichtige Bedeutung für nicht mobile Entwöhnungswillige und für jene, die in ihrer Wohnortnähe über kein professionelles Entwöhnungsangebot verfügen. Für Jugendliche bietet sie einen zusätzlichen Anreiz durch die Sicherheit der Anonymität.

\section{Beratungsangebote im Internet}

Beratungsmaßnahmen im Internet werden inzwischen in fast allen Ländern angeboten. Über Erfolgsraten lassen sich bisher keine Aussagen treffen.

\subsubsection{Medikamentöse Therapie der Tabakabhängigkeit}

D Das Verstehen der Vorteile und Grenzen der in der Tabakentwöhnung zur Verfügung stehenden Therapeutika bietet die Basis für einen wichtigen Beitrag zur individuellen Gesundheit und zur Volksgesundheit.

Es ist evidenzbasiert, dass die Kombination von nichtmedikamentösen und medikamentösen Interventionen die Effekte nicht nur addieren, sondern auch multiplizieren kann. Die Effektivität von allen Formen nichtmedikamentöser Therapien wird durch medikamentöse Maßnahmen in etwa verdoppelt! Je intensiver die nichtmedikamentöse Maßnahme, desto höher ist das Ausmaß der Verbesserung des Gesamteffekts.

Die Hersteller von First-line-Produkten in der Tabakentwöhnung empfehlen eine medikamentöse Unterstützung lediglich für Raucher, die mindestens 10 Zigaretten pro Tag konsumieren. In der Empfehlung des englischen National Institute for Clinical Excellence (NICE 2002) wird diese Einschränkung nicht bestätigt. Damit wird unter anderem der erheblichen Anzahl von reduziert Rauchenden Rechnung getragen, die zwar weniger als $10 \mathrm{Zi}$ garetten konsumieren, jedoch ihre Nikotinaufnahme und Aufnahme von toxischen Substanzen infolge des kompensierten Rauchens nicht ausreichend reduziert haben.

Die Wahl unter den nicht kontraindizierten Therapeutika kann den Patienten überlassen werden, jedoch nicht die Anfangsdosierung! Sollte sich allerdings die Anfangsdosierung als zu hoch erweisen, kann der Patient eine Reduktion selbst vornehmen. Die Dauer der medikamentösen Unterstützung sollte mit dem Entwöhnungswilligen gemeinsam entschieden werden.

\section{Nikotinersatztherapie (NET)}

Je stärker die empfundene Entzugssymptomatik ist, desto niedriger ist die Abstinenzrate. Viele experimentelle und klinische Studien haben gezeigt, dass die Entzugssymptome bei Rauchern auf Nikotinmangel zurückzuführen sind. Die Nikotinersatztherapie vermag die Entzugssymptome erheblich zu lindern.

D Die Nikotinersatztherapie ist eine First-line-Therapie in der Tabakentwöhnung.

Viele Patienten schrecken vor NET zurück, weil sie in deren Verordnung einerseits und dem Entschluss, die Droge Nikotin per Zigaretten nicht mehr zu sich zu nehmen, andererseits einen Widerspruch sehen. Hier ist professionelle Aufklärung und Beratung hilfreich:

- Medizinisches Nikotin enthält nicht die etwa 45 giftigen und 45 krebserregenden Substanzen des Zigarettenrauchs.

- Die schnellsten medizinischen Nikotinpräparate benötigen Minuten, Nikotin per Zigarettenrauch dagegen nur wenige Sekunden, um im Gehirn wirksam zu werden. Da jedoch das Abhängigkeitspotenzial der Drogen umso stärker ist, je schneller diese das Gehirn erreichen, besitzen auch die schnellen Nikotinersatzpräparate lediglich ein geringes Abhängigkeitsrisiko.

Die häufigsten Fehler in der NET liegen in einer zu niedrigen Dosierung, der kurzen Anwendung oder im Einsatz als Monotherapie, wenn eine Kombination erforderlich 
wäre. Eine Kombination eines kurzwirksamen Präparats (z. B. Kaugummi) mit einem langwirksamen (Pflaster) ist indiziert, wenn bei einer höhergradigen Tabakabhängigkeit tagsüber unregelmäßig stärkeres »craving" auftritt. Die Kombinationstherapie Nikotinkaugummi und Nikotinpflaster weist nach 12 Monaten eine signifikant höhere Abstinenzrate auf als eine der Applikationsformen alleine.

Eine längere und höher dosierte NET ist indiziert bei einer stärkeren Abhängigkeit, starken Entzugssymptomen bei vorausgehender Abstinenz, Gewichtszunahme bei vorausgehenden Abstinenzversuchen und einer Depression bei vorausgegangener Abstinenz. Im letzteren Fall sollte ausnahmsweise die Reduktionsvorgehensweise der Schlusspunktvorgehensweise vorgezogen werden.

Der Einsatz der schnellwirksamen Nikotinersatzpräparate ist eindeutig überlegen

- zur Gewichtskontrolle bei nichtakzeptabler Gewichtszunahme,

- für die Kombination mit Nikotinpflaster oder Bupropion,

- in rezidivierenden Krisensituationen infolge von »craving«.

Wechselwirkungen der NET, wie z.B. schnellere Metabolisierung von Salbutamol oder verlangsamte Metabolisierung von Theophyllin und Koffein, sind vor der Verordnung von NET zu beachten. Dieses gilt auch für die relativen Kontraindikationen von NET.

NET ist sicher bei stabiler Angina pectoris. NET sollte bei instabilen kardiovaskulären Erkrankungen, in der Schwangerschaft, während des Stillens und bei Jugendlichen unter 18 Jahren nur dann verordnet werden, wenn ansonsten keine Abstinenz zu erzielen ist bzw. eine starke Entzugssymptomatik (z. B. auf Intensivstationen) vermieden werden muss. Für diese Indikationen sind kurzwirksame Nikotinersatzpräparate wie Nikotinkaugummi, Lutsch-, Sublingualtabletten, Nikotinnasenspray oder Nikotininhalator zu bevorzugen, da für den Fall, dass die NET nicht gut vertragen wird, ihre Resorption sehr schnell beendet werden kann. Nach Entfernen eines Nikotinpflasters wird jedoch aus dem Hautdepot noch eine Zeit lang Nikotin in die Blutbahn abgegeben.

Es gibt keine Beweise dafür, dass Patienten mit bestimmten Krankheiten besser oder schlechter auf NET ansprechen als andere. Nikotinkaugummi ist als medikamentöse Unterstützung beim reduzierten Rauchen zugelassen (s. oben).

\section{Bupropion (Zyban ${ }^{\circledR}$ )}

( Bupropion ist verschreibungspflichtig und ebenfalls ein First-line-Therapeutikum in der Tabakentwöhnung.

Die Wirkungsweise besteht in der Hemmung der Wiederaufnahme von Noradrenalin und Dopamin und dadurch einer Imitation der Wirkung des Nikotins im Nucleus accumbens (vermuteter Wirkmechanismus). Die Entzugssymptomatik in der Abstinenzphase wird herabgesetzt. Die antagonistische Wirkung auf die nikotinischen Rezeptoren könnte den rückfallpräventiven Effekt von Bupropion erklären. Als zusätzliche Wirkung kommt es zu einer Herabsetzung der Gewichtszunahme während der Einnahme. Es wurde in den Studien auch ein »aversiver Effekt « in Bezug auf das Rauchen beobachtet.

Bupropion ist in den USA als atypisches Antidepressivum im Handel. Seine Wirksamkeit in der Tabakentwöhnung ist jedoch unabhängig von seiner antidepressiven Eigenschaft. Die Einnahme von Bupropion sollte nach Möglichkeit 8 Tage vor dem Rauchstopp beginnen. Eine maximale Einzeldosis von $150 \mathrm{mg}$ und eine Tageshöchstdosis von $300 \mathrm{mg}$ müssen eingehalten werden. Bei leichter oder mittelschwerer Leber- und/oder Nierenfunktionsstörung wie auch bei älteren Patienten wird die einmalige Einnahme von täglich $150 \mathrm{mg}$ empfohlen.

Die Dauer der Anwendung ist individuell und richtet sich nach der Dauer der Entzugssymptomatik: Es wird jedoch ein Minimum von 7-9 Wochen Therapie empfohlen. Wird bis zur 7. Behandlungswoche keine Abstinenz erzielt, sollte die Behandlung abgebrochen werden.

Bupropion kann mit NET kombiniert werden. Über die Therapie mit Bupropion bei unter 18-Jährigen liegen bisher nicht ausreichende Daten vor. Bupropion ist für Schwangere und Stillende nicht zugelassen.

In Bezug auf die Wechselwirkungen von Bupropion ist zu beachten, dass es ein schwacher Inhibitor der Cytochromoxidase P450 (Isoenzym 2D6) ist. Deshalb ist die Anpassung der Dosierung von Medikamenten, die ebenfalls über CYP450 metabolisiert werden, notwendig.

An Nebenwirkungen sind Krampfanfälle (0,1\% in der Dosierung von $300 \mathrm{mg} / \mathrm{Tag})$, Schlafstörungen und abnorme Träume sowie Mundtrockenheit hervorzuheben. 48 h nach Rauchstopp kann es zu einer Erhöhung des Serumspiegels verschiedener Medikamente kommen, die mit einer Erniedrigung der Krampfschwelle einhergehen können (z. B. Theophyllin, Koffein, Kortison).

Die Kontraindikationen (u. a. Epilepsie, Essstörungen, medikamentöse Therapien, die zur Erniedrigung der zerebralen Krampfschwelle führen) sind unbedingt zu beachten. Bisher sind mehr als 40 Studien zu Bupropion publiziert worden. Bupropion zeigte sich auch außerhalb von Studien, wenn die Kontraindikationen streng eingehalten wurden, als ein First-line-Therapeutikum mit tolerabler Rate an unerwünschten Wirkungen. Es traten in einem großen Krankengut keine zerebralen Krampfanfälle auf.

\section{Vareniclin (Champix ${ }^{\circledR}$ )}

Als partieller Nikotinrezeptoragonist bindet Vareniclin wie auch Nikotin mit hoher Affinität an den neuronalen 
$\alpha_{4} \beta_{2}$-nikotinergen Acetylcholinrezeptoren. Vareniclin besitzt eine agonistische Komponente und stimuliert den Rezeptor, führt zur Freisetzung von Dopamin im Gehirn und vermittelt so den positiven, nikotinvermittelten Belohnungseffekt beim Rauchen. Die antagonistische Wirkung blockiert den Rezeptor, als dessen Folge werden Rauchverlangen und Entzugssymptome reduziert.

Es liegen Daten aus 5 multizentrischen randomisierten Doppelblindstudien mit insgesamt etwa 5000 Rauchern zur Wirksamkeit von Vareniclin vor. Als häufigste unerwünschte Wirkung wurde mit rund 30\% Übelkeit genannt. Bei über 10\% der Teilnehmer traten Schlafstörungen, Kopfschmerzen und abnorme Träume auf. Im Jahre 2008 publizierte Ergebnisse (Stapleton et al. 2007) bestätigen, dass unerwünschte Wirkungen unter Vareniclinbehandlung wie Übelkeit und Schlafstörungen im Vergleich zur Nikotinersatztherapie signifikant häufiger auftreten. Im Internet wurden im Jahr 2008 Bedenken der FDA in Bezug auf psychiatrische unerwünschte Wirkungen mitgeteilt (http:// www.fda.gov/cder/drug/advisory/varenicline.htm).

D Vareniclin ist derzeit für die Behandlung von Schwangeren, Stillenden und unter 18jährigen Jugendlichen nicht zugelassen.

Vareniclin verändert die Pharmakogenetik von Bupropion nicht. In einer vergleichenden Studie erwies sich Vareniclin wirksamer als Bupropion. Bei gleichzeitiger Verabreichung von Vareniclin mit NET kam es zur Verstärkung der NET-Nebenwirkungen wie Übelkeit, Kopfschmerzen, Benommenheit und Müdigkeit. Soweit bekannt interagiert Vareniclin nicht mit anderen klinisch bedeutsamen Wirkstoffen. Die Anwendung von Vareniclin zur Tabakentwöhnung erhöht die Abstinenzwahrscheinlichkeit gegenüber Placebo langfristig etwa auf das 3 -fache. Nach Lancaster und Mitarbeitern (2008) ist Vareniclin mindestens so wirksam wie NET und Bupoprion oder Nortryptilin.

Vareniclin zeigt sich als wirksames und nach Datenlage auch gut verträgliches Medikament in der Tabakentwöhnung und eröffnet aufhörwilligen Rauchern eine neue Möglichkeit in die Rauchfreiheit. Vareniclin ist in den USA und in der EU bereits zugelassen und steht seit März 2007 auch in Deutschland zur Verfügung.

\section{Immunologische Ansätze in der Behandlung der Tabakabhängigkeit}

Im Gegensatz zu derzeitigen medikamentösen Therapieformen haben die immunologischen Ansätze zur Behandlung der Tabakabhängigkeit nicht das Gehirn sondern die Droge Nikotin als Zielobjekt. Dieser Ansatz beinhaltet die Impfung mit spezifischen Nikotinantikörpern, die im Serum eine Bindung mit Nikotin eingehen. Daraus resultiert eine Erniedrigung der Verteilung von Nikotin im Gehirn und eine Erhöhung der Nikotineliminationshalbwertszeit im Serum. Durch die Anbindung von Antikörpern an Nikotin im Blut kann die Blut-Hirn-Schranke nicht mehr passiert werden. Die Anwendung von verschiedenen Vakzinen in Tierversuchen zeigte eine signifikante Herabsetzung der durch Nikotin ausgelösten Abhängigkeit.

D Die Erfolgsrate des immunologischen Ansatzes hängt vorwiegend von der Höhe des individuell erreichten Serumantikörperspiegels ab.

Phase I klinischer Studien mit Nikotinvakzinen haben zu keinen schweren unerwünschten Wirkungen bei den Probanden geführt. Obwohl die bisherigen Daten aus den wenigen Untersuchungen zeigen, dass die Verabreichung von Vakzinen die Tabakabstinenz begünstigt, sind weitere Untersuchungen erforderlich, um eine abschließende Einschätzung zuzulassen.

3 verschiedene Antikörperprovokationen sind bislang in Phase-II-Studien untersucht worden. Als Träger wurden bislang das Exotoxin von Pseudomonas aeruginosa und Choleratoxin B verwendet. 4-6 Boosterinjektionen pro Person wurden durchgeführt. Bei den Probanden, die effektive Titer erreicht haben, führte die Behandlung zu Entzugssymptomen. Durch die Aktion außerhalb des zentralen Nervensystems ist die aktive Immunisierung mit weniger Nebenwirkungen behaftet als die bisherigen medikamentösen Therapien. Die Antikörper führen neben der Reduzierung des Verstärkereffekts von Nikotin im Gehirn auch zur Rückfallprävention im Falle eines »Ausrutschers» nach erfolgreicher Entwöhnung. Mit der Vakzinenbehandlung des Nikotinkonsums könnten in Zukunft die bekannten medikamentösen Therapien kombiniert werden.

\subsubsection{Spezielle Populationen}

\section{Schwangere}

Insbesondere sind für Schwangere die giftigen Substanzen im Tabakrauch wie CO, Zyanid, Kadmium und Blei von Bedeutung. Nikotin per Zigarette führt wegen höherer, schnell erreichter Konzentration zu Vasokonstriktion in der Plazenta und infolge Entwicklungsverzögerung des Fötus zur Schädigung. Wenn eine Schwangere im 1. Trimenon abstinent wird, sind für den Fötus bleibende Schädigungen meist nicht zu erwarten. Für Schwangere sollten eher kurzwirksame Nikotinersatzpräparate und kein Pflaster gewählt werden (s. oben).

Die einzige placebokontrollierte Studie mit NET bei Schwangeren ergab einen positiven Effekt auf das Gewicht der Neugeborenen. Die internationale Literatur berichtet über Anwendung von NET in der Schwangerschaft und Unsicherheit der Geburtshelfer bei dieser Therapieoption. Eine multizentrische Studie in England soll in den nächsten Jahren Klarheit schaffen, wie sich die bekannte Beschleunigung der Nikotinmetabolisierung bei 
Schwangeren auf die medikamentöse Unterstützung der Tabakentwöhnung auswirkt.

\section{Jugendliche Raucher}

Das typische Probieralter für Jugendliche liegt derzeit zwischen dem 11. und 15. Lebensjahr. Vom ersten Zug über das Inhalieren bis zum Auftreten von Symptomen einer Tabakabhängigkeit sind es nur wenige Monate. Die ersten "Meilensteine« werden bei Mädchen früher erreicht als bei Jungen. Symptome der Abhängigkeit treten noch vor dem täglichen Rauchen auf - wenn ein regelmäßiger Konsum einsetzt, sind viele bereits süchtig. Ein früher Tabakkonsum führt zudem mit einer höheren Wahrscheinlichkeit zu einem Alkohol- und Drogenkonsum (Marihuana, Kokain). Bei Jugendlichen, Eltern und Gesundheitsexperten muss daher dringend ein Umdenken erfolgen: Die Behandlung der Altersgruppe der 11- bis 15-jährigen Raucher sollte auch ein Abhängigkeits- bzw. Entzugsmanagement beinhalten.

NET (Nikotinpflaster und -kaugummi) hat sich bisher bei jugendlichen Rauchern als gut verträglich erwiesen. Im Rahmen einer amerikanischen Kohortenstudie wurden Schüler der 11. Klasse nach dem Konsum von NET befragt. 5\% der Jugendlichen haben Nikotinkaugummi oder -pflaster probiert oder zum Zeitpunkt der Befragung benutzt. Etwa 40\% der jugendlichen Exraucher benutzten NET bei ihrem Entwöhnungsversuch. 75\% der jugendlichen Aktivraucher wendeten NET aus anderen Gründen als Entwöhnung an (z. B. zum reduzierten Rauchen oder in Situationen, in denen nicht geraucht werden durfte).

\section{Ausblick}

Zur zukünftigen effektiveren Nutzung der verfügbaren Behandlungsmethoden der Tabakentwöhnung könnte die Kombination der verschiedenen Behandlungsmöglichkeiten beitragen. Eine bereits vor dem Rauchstopp einsetzende Behandlung und die Möglichkeit einer intensiveren Betreuung bei Bedarf sind weitere Eckpfeiler, um in der Zukunft die Effektivität zu steigern.

Qualitätsverbesserungen in der Tabakentwöhnung können zukünftig durch neue und billigere Medikamente und eine umfassendere verhaltenstherapeutische Behandlung erzielt werden. Die neu eingeführten gesetzgeberischen Maßnahmen zum Nichtraucherschutz werden ebenfalls dazu beitragen, bestehende Hilfsangebote zur Tabakentwöhnung besser zu nutzen.

\section{Literatur}

Batra A, Schütz CG, Lindinger P (2006) Tabakabhängigkeit. In: Schmidt LG, Gaspar M, Falkai P, Gaebel W (Hrsg.) Evidenzbasierte Suchtmedizin. Behandlungsleitlinie Substanzbezogene Störungen. Deutscher Ärzteverlag, Köln, S. 91-142
Bölcskei PL (2004) Tabakentwöhnung: Kritische Bestandsaufnahme und Chancen für die Zukunft. Pneumologie 58:137-139

Britton J (Hrsg.) (2004) ABC of Smoking cessation. BMJ Books, Blackwell, Oxford

Cahill K, Stead LF, Lancaster T (2007) Nicotine receptor partial agonists for smoking cassation. Cochrane Database of Systematic Reviews Issue 1. Wiley, Chichester

Cokkinides V et al. (2006) Progress and Opportunities in Tobacco Control. CA Cancer J Clin 56:135-142

Cornuz J, Humair JP, Zellweger JP (2004) Tabakentwöhnung 1. Teil: Wie es geht und was es bringt. Schweiz Med Forum 4:764-770

Cornuz J, Humair JP, Zellweger JP (2004) Tabakentwöhnung 2. Teil: Empfehlungen für die tägliche Praxis. Schweiz Med Forum 4:792-805

Deutsches Krebsforschungszentrum (Hrsg.) (2003) Passivrauchende Kinder in Deutschland - Frühe Schädigungen für ein ganzes Leben. DKFZ, Heidelberg

Deutsches Krebsforschungszentrum (Hrsg.) (2003) Tabaksteuererhöhungen - Fakten und Argumente. DKFZ, Heidelberg, 2003

Deutsches Krebsforschungszentrum (Hrsg.) (2005) Passivrauchen - ein unterschätztes Gesundheitsrisiko. DKFZ, Heidelberg, 2005

Fiore MC et al. (2000) Treating Tobacco Use and Dependence. Clinical Practice Guideline, U.S. Department of Health and Human Services, Washington DC

Fletcher C, Peto R (1977) The natural history of chronic airflow obstruction. Brit Med J 1:1645-1648

Gervais A et al. (2006) Milestones in the natural course of onset of cigarette use among adolescents. CMAJ 175(3):255 ff

Gonzales D et al. (2006) Varenicline, an $\alpha_{4} \beta_{2}$ Nicotinic Acetylcholine Receptor Partila Agonist, vs Substained-Release Bupropion and Placebo for Smoking Cessation. JAMA 296(1):47-55

Henley SJ et al. (2007) Tabacco-related disease mortality among men who switched from cigarettes to spit tabacco. Tabacco-Control 16(1):22-8

Hering T, Bölcskei PL (2002) Raucher in der Praxis entwöhnen. Ein neues Programm ist in über $50 \%$ erfolgreich. MMW-Fortschr Med 18:50-51

Hughes JR, Stead LF, Langester T (2007) Antidepressants for smoking cessation. Cochrane Database of Systematic Reviews, Issue 1, Wiley, Chichester

Lancaster T, Stead L, Cahill K (2008) An update on therapeutics for tobacco dependence. Expert opinion on pharmacotherapy 9:15-22

LeSage MG, Keyler DE, Pentel PR (2006) Current status of immunologic approaches to treating tobacco dependence: vaccines and nicotine-specific antibodies. AAPS J 8(1):E65-75

Lob-Corzilius T (2006) Gesundheitsgefahren durch Tabakrauchbelastung von Kindern. Nicorette Science 3:2-4

Maurer P, Bachmann MF (2007) Vaccination against nicotine: an emerging therapy for tobacco dependence. Expert opinion on investigational drugs 16:1775-1783

Miller WR \& Rollnick S (2004) Motivierende Gesprächsführung. Lambertus: Freiburg

National Institute for Health and Clinical Excellence (NHS) (2002) Smoking cessation - bupropion and nicotine replacement therapy. Guidance type: Technology Appraisal TA39

Neubauer $S$ et al. (2006) Mortality, morbidity and costs attributable to smoking in Germany: update and a 10-year comparison. Tobacco Control 15:464-471

ÖGAM (2007) Konsensus Statement: Raucherentwöhnung in der allgemeinmedizinischen Praxis. 2. erw. Aufl. Intern Z ärztl Fortb 3

Österreichische Gesellschaft für Pneumologie (ÖGP) (2005) Standards der Raucherentwöhnung. Konsensus der ÖGP. Wiener Klinische Wochenschrift (Suppl.2) 117:1-18

Prochaska JO, DiClemente CC, Norcross JC (1992) In search how people change. Applications to addictive behaviors. American Psychologist 47(9):1102-1114 
Raupach T, Nowak D, Hering T, Batra A, Andreas S (2007) Rauchen und pneumologische Erkrankungen, positive Effekte der Tabakentwöhnung. Pneumologie 61:11-14

Silagy C, Lancaster T, Stead LF, Mant D, Fowler G (2005) Nicotine replacement therapy for smoking cessation (Review). Cochrane Library Issue 4, Wiley, Chichester

Stapleton JA, Watson L, Spirling LI, Smith R, Milbrandt A, Ratcliffe $M$, Sutherland $G$ (2008) Varenicline in the routine treatment of tobacco dependence: a pre-post comparison with nicotine replacement therapy and an evaluation in those with mental illness. Addiction 103:146-154

Statistisches Bundesamt (Hrsg.) (2006) Leben in Deutschland. Haushalte, Familien und Gesundheit - Ergebnisse des Mikrozensus 2005. Statistisches Bundesamt, Wiesbaden

Stead LF, Lancaster T (2005) Group behaviour therapy programmes for smoking cessation. Cochrane Database of Systematic Reviews: Reviews, Issue 2, Wiley, Chichester

Stead LF, Lancaster T (2007) Interventions to reduce harm from continued tobacco use. Cochrane Database of Systematic Reviews, Issue 4, Wiley, Chichester

Tashkin D, Kanne R, Bailey W et al. (2001) Smoking cessation in patients with chronic obstructive pulmonary disease: a double blind placebo-controlled randomised trial. Lancet 357:1571-1575

Thürauf N (2005) Der Schalter zur Sucht: Neue Einblicke in die Tabakabhängigkeit. Nicorette Science 3:2-4

Tverdal A, Bjartveit K (2006). Health consequences of reduced daily cigarette consumption. Tobacco Control 15:472-480

Wennike P, Danielsson T, Landfeldt B, Westin A, Tonnesen P (2003) Smoking reduction promotes smoking cessation: results from a double blind, randomized, placebo-controlled trial of nicotine gum with 2-year follow-up. Addiction 98:1395-1402

West R, Sohal T (2006) »Catastrophic « pathways to smoking cessation: findings from national survey. BMJ 332:458-460 